\author{
WALDEN \\ UNIVERSITY \\ A higher degree. A higher purpose.
}

Walden University ScholarWorks

Walden Dissertations and Doctoral Studies

2018

\title{
Nurse Practitioners' Experiences with Role Transition: Supporting the Learning Curve Through Preceptorship
}

Viktoriya Y. Pleshkan

Walden University

Follow this and additional works at: https://scholarworks.waldenu.edu/dissertations

Part of the Adult and Continuing Education Administration Commons, Adult and Continuing Education and Teaching Commons, and the Nursing Commons

This Dissertation is brought to you for free and open access by the Walden Dissertations and Doctoral Studies Collection at ScholarWorks. It has been accepted for inclusion in Walden Dissertations and Doctoral Studies by an authorized administrator of ScholarWorks. For more information, please contact ScholarWorks@waldenu.edu. 


\title{
Walden University
}

\author{
College of Health Sciences
}

This is to certify that the doctoral dissertation by

\begin{abstract}
Viktoriya Pleshkan,
has been found to be complete and satisfactory in all respects, and that any and all revisions required by the review committee have been made.

Review Committee

Dr. Leslie Hussey, Committee Chairperson, Nursing Faculty

Dr. Donna Bailey, Committee Member, Nursing Faculty

Dr. Corinne Wheeler, University Reviewer, Nursing Faculty
\end{abstract}

\section{Chief Academic Officer}

Eric Riedel, Ph.D.

Walden University

2018 


\begin{abstract}
Nurse Practitioners' Experiences with Role Transition: Supporting the Learning Curve Through Preceptorship

by

Viktoriya Pleshkan
\end{abstract}

MSN, FNP, University of Saint Francis, 2006

BSN, Indiana University_Purdue University Fort Wayne, 2003

\author{
Dissertation Submitted in Partial Fulfillment \\ of the Requirements for the Degree of \\ Doctor of Philosophy \\ Nursing
}

Walden University

August 2018 


\begin{abstract}
The nurse practitioner (NP) role transition is difficult and ill-supported before and after graduation. Although preceptorships are essential in supporting a NP's role transition, there is a paucity of data on student and novice NPs' experiences with their role transition and how it is supported through preceptorships. The purpose of this hermeneutic phenomenological study was to understand and describe the meaning of NPs' role transition experiences while in a preceptorship before and after graduation. Schlossberg's transition theory and the cognitive apprenticeship models were used to guide the exploration of concepts of role transition and preceptorship. One face-to-face, 3 Skype, and 12 phone interviews were conducted with NPs who were currently practicing in the NP role ( $>3$ months but $<5$ years) in those states that require collaborative practice agreement. Transcribed interviews were coded using Van Manen's analysis strategies. Findings suggested that NPs' difficult transition to practice was related to their preceptorship support in NP school and in NP work settings. The data showed ill-supported preceptorships when in NP programs. Lack of preceptorships after the graduation was accompanied by employers' expecting novice NPs to function at an expert level, frequently with minimal guidance. Understanding how NPs transition to practice and how their role transition is supported through preceptorships can be used to inform NP educators and organizations that hire NPs of the changes needed to strengthen NPs' role transition. These findings may promote positive social change by guiding stakeholders to improve the NP role transition, which could, in turn, increase NPs' autonomy, retention, and job satisfaction.
\end{abstract}


Nurse Practitioners' Experiences with Role Transition: Supporting the Learning Curve Through

$$
\text { Preceptorship }
$$

by

Viktoriya Pleshkan

MSN, FNP, University of Saint Francis, 2006

BSN, Indiana University Perdue University of Fort Wayne

\author{
Dissertation Submitted in Partial Fulfillment \\ of the Requirements for the Degree of \\ Doctor of Philosophy \\ Nursing
}

Walden University

August 2018 


\section{Dedication}

This work is dedicated to my family, who supported me though this intellectual journey. I would not have done this without your constant support and encouragement. To my husband, Vadim, who encouraged me to start the program and I am grateful for that. To my parents Alla and Jeff, who were patient all the way to finish line. To my children, Gina and Ilya, who were helpful and understanding during the years of my life I spent on writing my dissertation. Most importantly, Glory to God as great things can only be done with God's guidance. 


\section{Acknowledgments}

I would like to acknowledge and thank my dissertation committee for their consistent guidance throughout my dissertation journey. I am grateful to Dr. Hussey who provided gentle but firm directions to not only make my study significant but also doable. Dr. Leslie Hussey, your thoughtful feedback was always timely and I am thankful for that. Thank you to Dr. Donna Bailey for guiding me through the qualitative methodology. I appreciate your expertise. Thank you to Dr. Corrine Wheeler for evaluating my work. I am also grateful to Dr. Margaret Spillett. The "forest and the trees" of the qualitative methodology that you shared with me were greatly appreciated. Your insight was invaluable. I would like to also acknowledge and thank the nursing organization that assisted me with the distribution of my research flyers during the recruitment stage. Lastly, I am grateful to my study participants who shared their stories with me. 


\section{Table of Contents}

List of Tables ..................................................................................................... vii

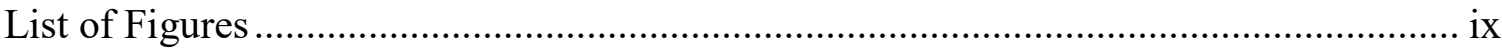

Chapter 1: Introduction to the Study .....................................................................

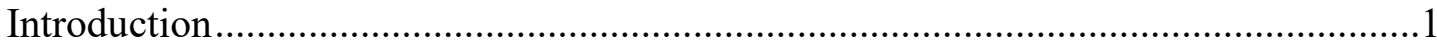

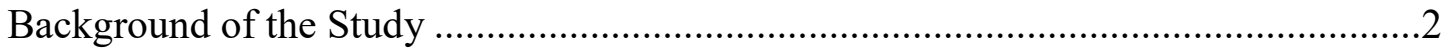

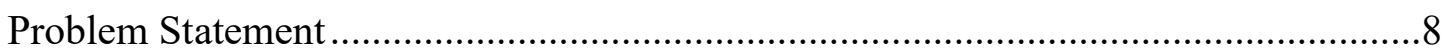

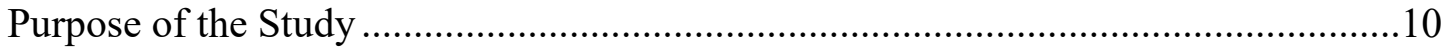

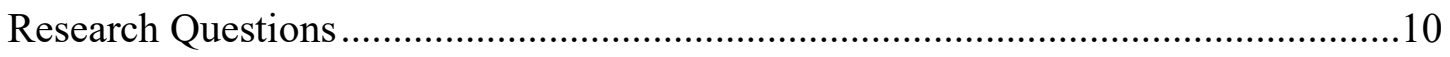



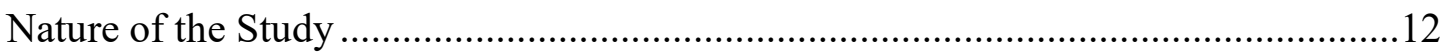

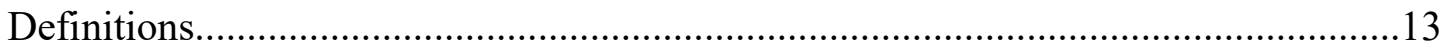

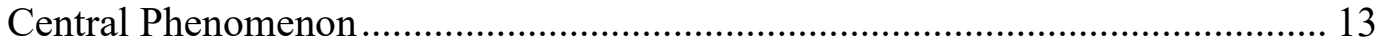

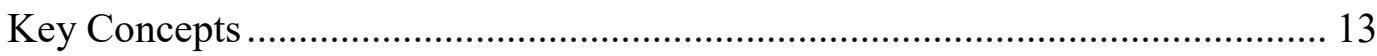

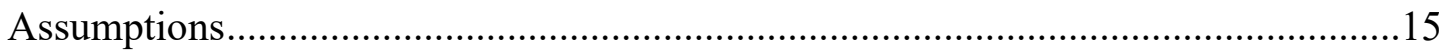

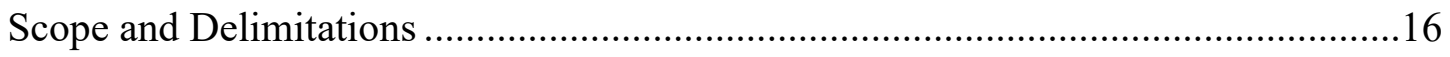

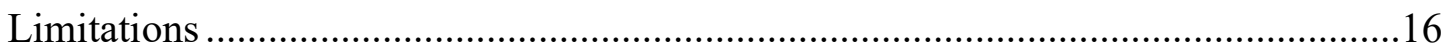

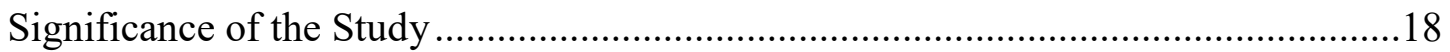

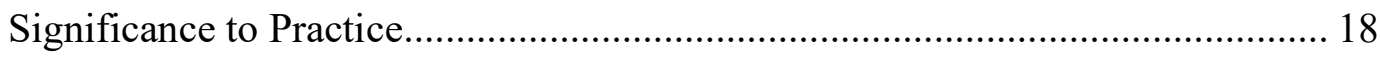

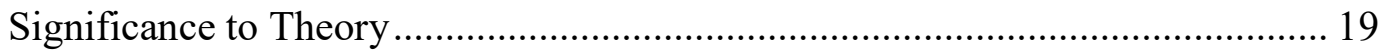

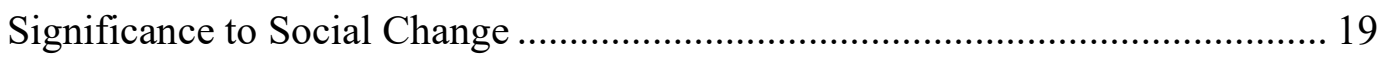

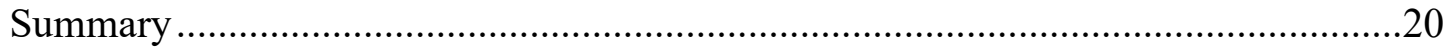


Chapter 2: Literature Review ................................................................................21

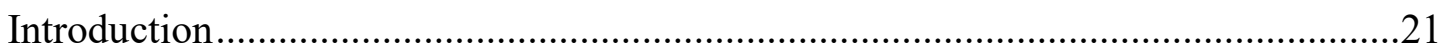

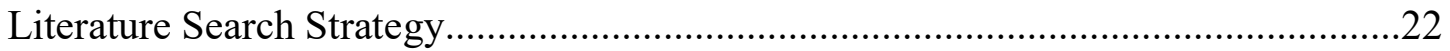

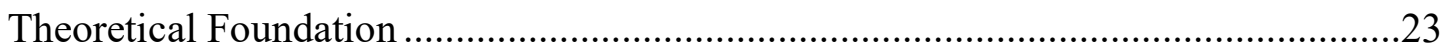

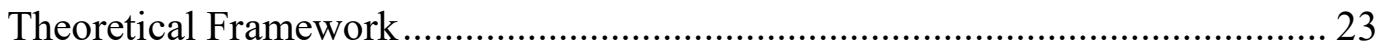

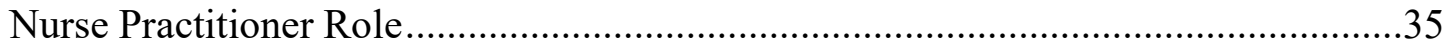

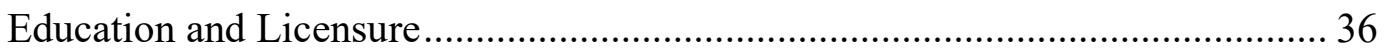

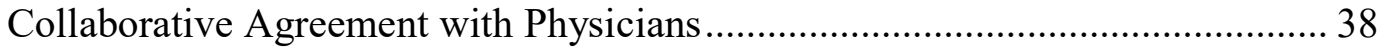

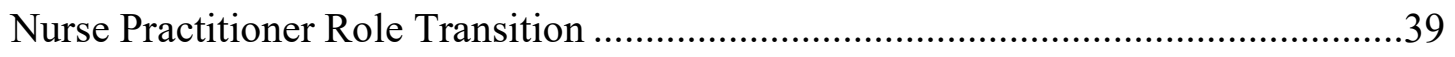

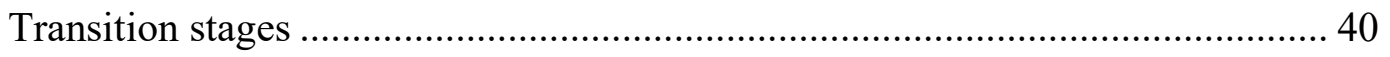

Transition, Preceptorship, and Resources ...............................................................42

Transition Attribute: Role Learning..................................................................... 43

Transition Attribute: Autonomy and New Responsibilities.................................. 46

Transition Attribute: Reconciling Two Identities ................................................. 49

Transition Attribute: Conflicting Feelings............................................................ 53

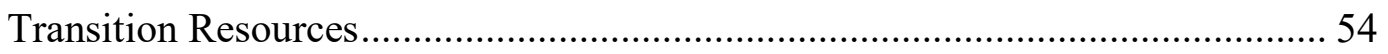

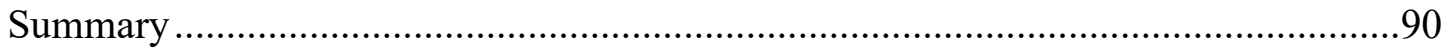

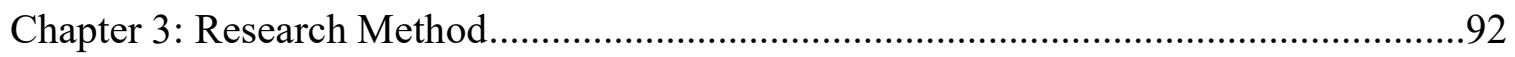

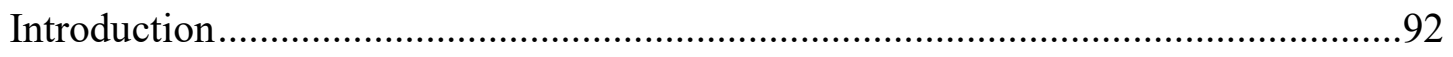



Philosophical Assumptions and Research Design ................................................. 93

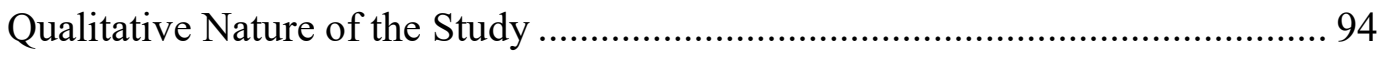




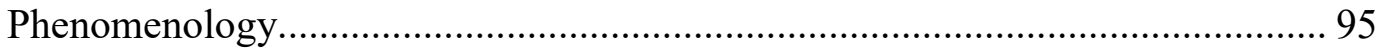

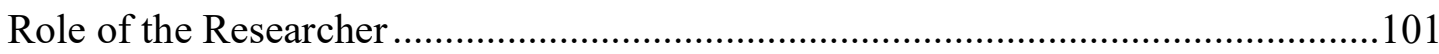

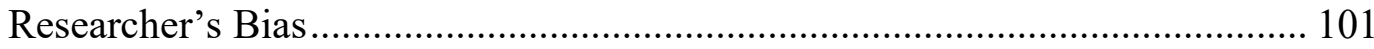

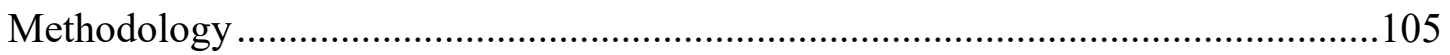

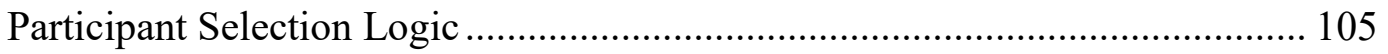

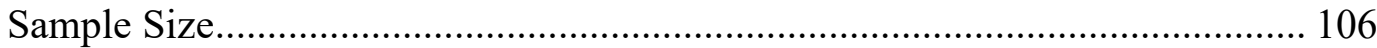

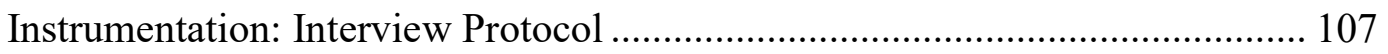

Procedures for Recruitment, Participation, and Data Collection ......................... 109

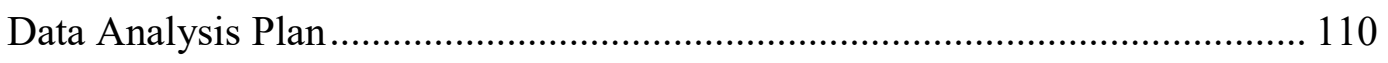

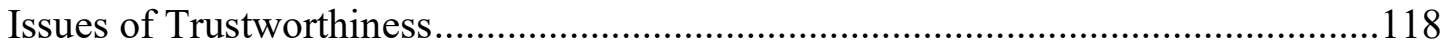

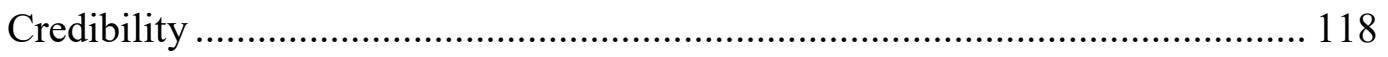

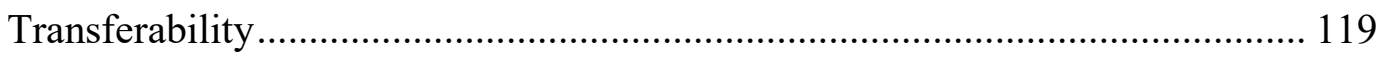

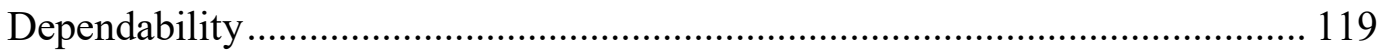

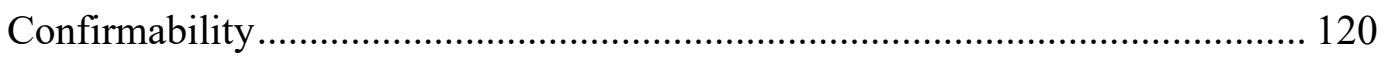

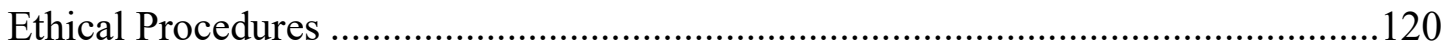

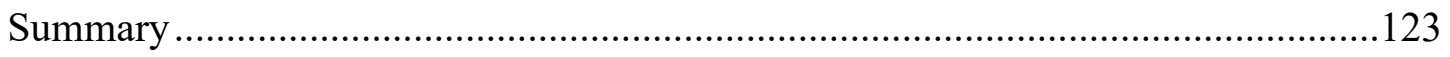

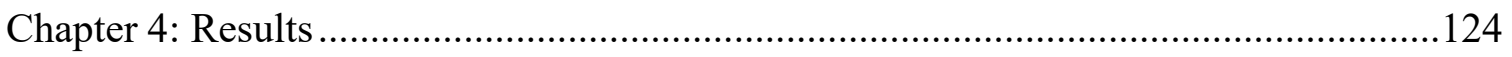

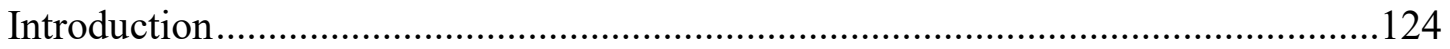

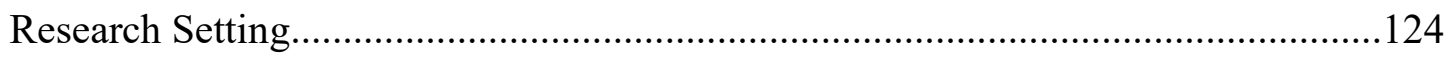

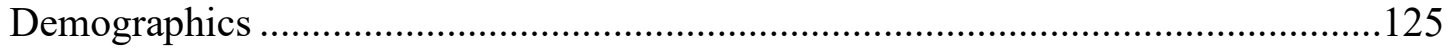

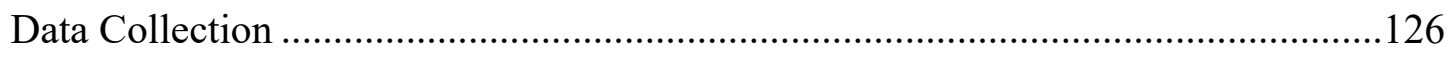

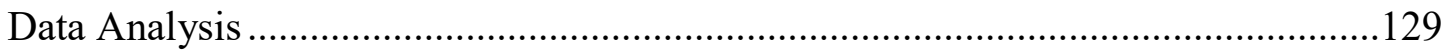


Van Manen's Six Analysis Procedures............................................................. 129

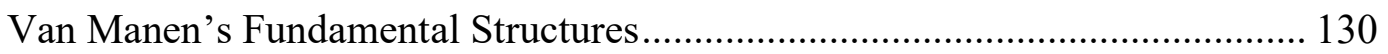

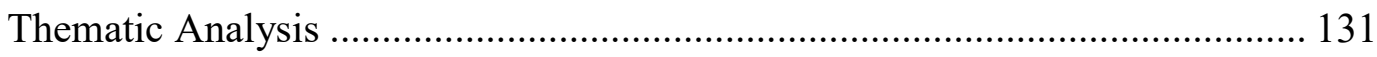

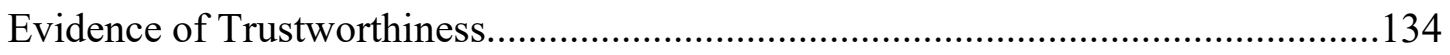

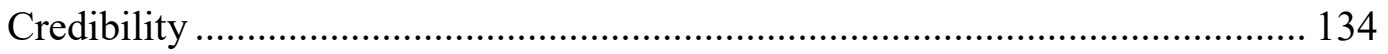

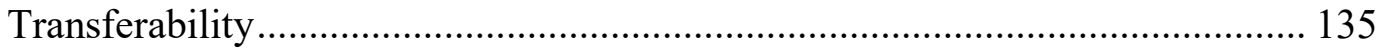

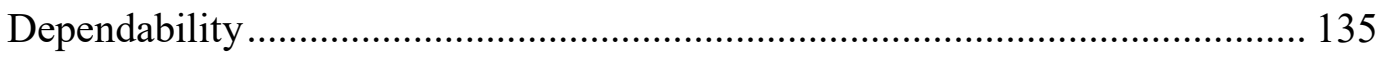

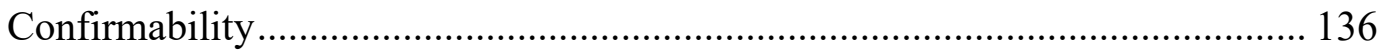

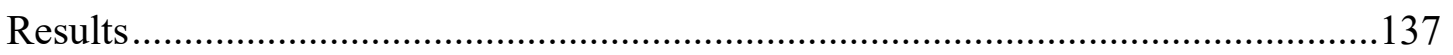

Theme I: Transition Preparation and Learning ................................................... 137

Theme II: Preceptorship During Role Transition and Learning .......................... 153

Theme III: Learning to Care for Complex Patients .............................................. 188

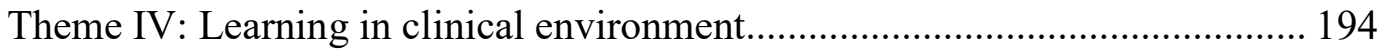

Theme V: Transitioning to a Greater Autonomy and new Responsibilities....... 228

Theme VI: Embracing the Role and Identity Confusion .................................... 248

Theme VII: Transition Reactions. Excited and Unfulfilled................................ 270

Chapter 5: Discussion, Conclusions, and Recommendations ..........................................286

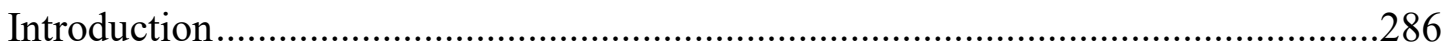

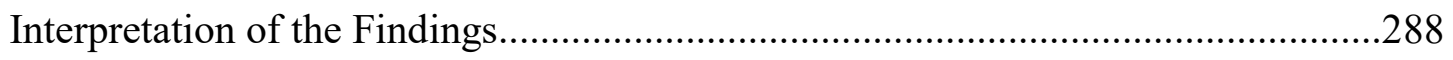

Theme I: Transition Preparation and Learning. Pre-graduation Meet and Greet Clinical Orientation to Post-Graduation "Winging it"

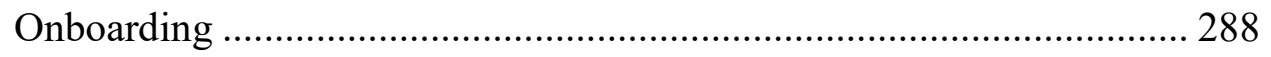


Theme II: Preceptorship During Role Transition and Learning. Pregraduation “Hands-off” Learning From Busy Preceptors to Postgraduation Self-Teaching. 291

Theme III: Learning to Care for Complex Patients. Pre-graduation Random and Fragmented Clinical Experiences to Post-Graduation Complexities of a Real Life 295

Theme IV: Learning in Clinical Environment. Pre-Graduation "Begging" for Preceptors and Sites to Post-Graduation "Collaborator on Paper." 296

Theme V: Transitioning to a Greater Autonomy and new Responsibilities. Pre-Graduation "Attached to the Preceptor's Hip" to PostGraduation "Jack of all Trades.” 300

Theme VI: Embracing the Role and Identity Confusion. Change From the "Notify the Provider Mindset" 302

Theme VII: Transition Reactions. Changing from "Excited" to "Scared and Unfulfilled." 304

Theoretical Interpretation of the Findings ................................................... 305

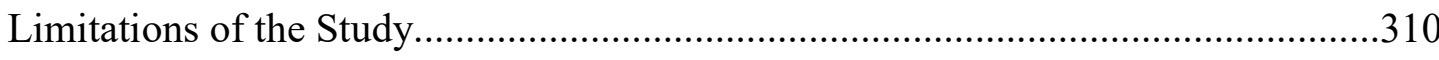

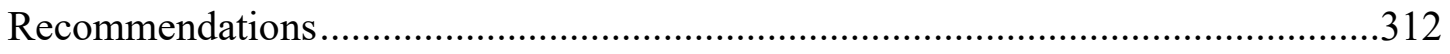

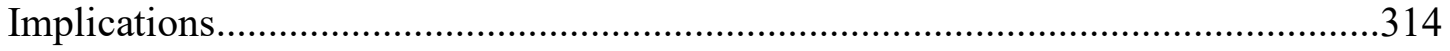

Implications for Organizations and Physicians That Hire NPs ....................... 314

Implications for NP Educators ............................................................. 315 


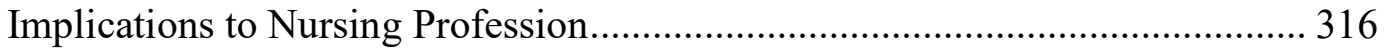

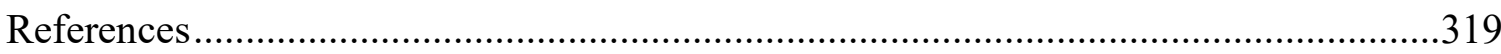

Appendix A: Demographic Information Form ……………..........................................340

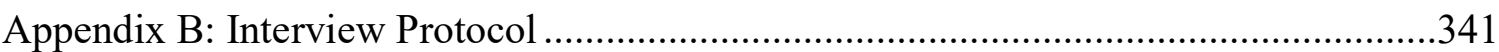

Appendix C: Participants’ Demographic Data …………..................................................343

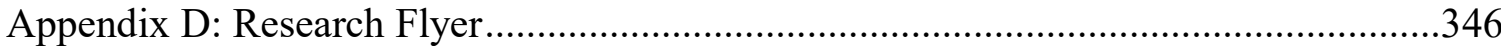

Appendix E: Pre-Graduation Transition Stage Analysis...............................345

Appendix F: Post-Graduation Transition Stage Analysis.............................346 


\section{List of Tables}

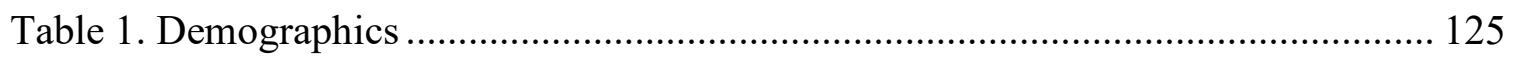

Table 2. Theme I: Pre-graduation: Transition Preparation and Learning ...................... 140

Table 3. Theme I: Post-graduation: Transition Preparation and Learning ..................... 145

Table 4. Theme I: Post-graduation: Transition Preparation and Learning. Onboarding. 148

Table 5. Theme II: Pre-Graduation: Preceptorship During Role Transition and Learning.

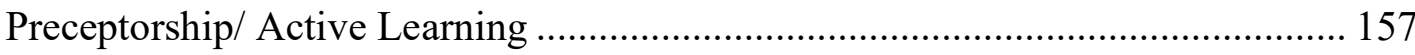

Table 6. Theme II: Pre-graduation: Preceptorship during role transition and learning.

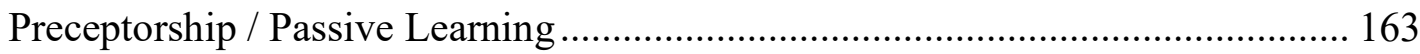

Table 7. Theme II: Pre-graduation: Preceptorship during role transition and learning.

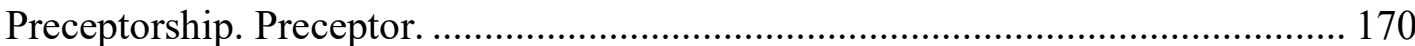

Table 8. Theme II: Post-graduation: Preceptorship During Role Transition and Learning. Novice NPs’ Learning. Self-Directed Learning................................................. 178

Table 9. Theme II: Post-graduation: Preceptorship During Role Transition and Learning. Preceptors' Teaching. 185

Table 10. Theme III: Post-graduation: Transitioning to Complex Pts and Learning. Learning Complexities. 190

Table 11. Theme III: Transitioning to Complex Pts and Learning Post-Graduation: Complexities of a Real World 194

Table 12. Theme IV: Pre-graduation: Transitioning in clinical environment and learning. Space. Clinical Sites. Finding clinical sites and clinical sites quality 203 
Table 13. Theme IV: Pre-graduation: Transitioning in Clinical Environment and Learning. Relationships and People

Table 14. Theme IV: Post-graduation: Transitioning in Clinical Environment and Learning. People. 216

Table 15. Theme IV: Post-graduation: Transitioning in Clinical Environment and Learning. Relationships and Communication 222

Table 16. Theme IV: Post-graduation: Transitioning in clinical environment and learning. Space. Proximity to other professionals.. Learning Alone. Never Alone. Familiarity Own Space. 228

Table 17. Theme V: Pre-graduation: Transitioning to a Greater Autonomy and new Responsibilities. Students' Autonomy and Preceptors. 236

Table 18. Theme V: Post-Grad: Transitioning to a Greater Autonomy and new Responsibilities. Wide Scope. Autonomy in decision making. Administrative Duties. 244

Table 19. Theme VI. Embracing the Role and Identity Confusion. .............................. 260

Table 20. Theme VII: Pre-graduation: Transition reactions. ..................................... 271

Table 21. Post-graduation: Transition reactions. ..................................................... 273

Table 22. Post-graduation: Transition reactions and feelings.................................... 274 
List of Figures

Figure 1. Role transition and cognitive mentorship concepts ......................................... 34 
Chapter 1: Introduction to the Study

\section{Introduction}

Registered nurse $(\mathrm{RN})$ to nurse practitioner $(\mathrm{NP})$ role transition is characterized by significant changes in nurses' role, including increased levels of autonomy and new responsibilities of being a prescriber. Strategies proposed to support this difficult transition to practice include post-graduation residency programs, academia-practice partnerships (APP), and increased use of simulation laboratories. However, with only a few residencies and APPs available in the United States, and an inability to substitute complex real-life experiences using simulated experiences, NPs' transition to practice is ill-supported. The problem begins with the gap between NPs' education and the level of skills required at the start of NP practice.

To close the gap between education and practice, preceptorships have been used to support NPs' clinical learning, both before and after graduation. However, preceptorships used prior to graduation have not had major changes since the 1960 s and preceptorships used after NPs graduate are scarce (AACN, 2015; Giddens et al., 2014; LeFlore, \& Thomas, 2016). Additionally, little information is available about how NPs transition to their new role, while supported by a preceptorship (Jarrell, 2016; Poronsky, 2012). Most of NPs' clinical experiences are not observed; thus, NPs themselves can provide the most insight into the nature of their transition. The purpose of this study is to explore the NPs' experiences with their role transitions while in a preceptorship. Understanding how NPs transition to practice and how a preceptorship supports their transition may inform needed changes and policies to strengthen the transition. 
In this chapter, I cover the following topics: background, research problem, purpose, research question, theoretical framework, nature of the study, definitions of key concepts, assumptions, delimitations, limitations, and significance.

\section{Background of the Study}

NPs are RNs with an advanced nursing education and clinical training who are licensed to diagnose and prescribe in the United States (Coombs, 2015). In recent years, the demand for NPs grew due to the shortage of primary care (PC) services (Giddens et al., 2014). The need for adult primary care providers (PCP) was predicted to grow by $29 \%$ from 2005 to 2025 , leading to the projected shortage of up to 40,000 PCPs (Sroczynski \& Dunphy, 2012). The need for NPs grew due to the increased physician shortage, recognition of NPs abilities as advanced nursing providers, and reduced medical residents' hours (Keough, Arciero, \& Connolly, 2015; Roberts, Wheeler, Tyler, \& Padden, 2017). Indeed, the number of practicing NPs increased by more than 100,000 since 2009 with the current total number of practicing NPs exceeding 234,000 (American Association of Nurse Practitioners [AANP], 2017a). To accommodate this increased need, the number of NP programs also proliferated; the current number of programs in the United States is approximately 350 (AANP, 2017b). From 2010-2015, NP enrollment numbers grew 81\% (AACN, 2015). Because NPs obtain their skills through experiential learning, clinical education is the foundation of any NP program. However, even with the growing number of NP programs, the clinical training model has not had major changes in 45 years; it has become outdated and ineffective (AACN, 2015; Giddens et al., 2014; LeFlore, \& Thomas, 2016). 
The RN to NP role transition has been identified as difficult and stressful (Brown \& Olshansky, 1997; Flinter \& Hart, 2016; Steiner, McLaughlin, Hyde, Brown, \& Burman, 2008). Though many other medical professions offer residencies to their new graduates to close the gap between education and real-world practice, most NPs start practicing right after graduation (Hevesy, Aitchison, Ruiz, \& Bednar, 2016). The ill-supported clinical education during school is followed by the lack of transition support after graduation (AACN, 2015; Donley, Flaherty, Sarsfield, Burkhard, O'Brien, \& Anderson, 2014; Logan, Kovacs, \& Barry, 2015; Roberts et al., 2017; Webb, Lopez, \& Guarino, 2015; Brown \& Olshansky, 1997; Flinter \& Hart, 2016; Steiner et al., 2008). Employers expect newly graduated NPs to care for a wide range of complex patients. Poorly supported role transition after graduation is dangerous because it may cause novice NPs to practice outside their scope of practice for fear of losing a job. In fact, $47.7 \%$ of NPs felt that they were practicing outside their scope of practice during their first year of practice (Hart \& Bowen, 2016). Novice NPs expressed concerns about the lack of training to perform procedures, such as suturing, fracture care, X-ray readings, etc. (Jones, Kotthoff-Burrell, KassWolff, \& Brownrigg, 2015). Lack of clinical skills could cause NPs to struggle in their new role and thus undermine the value of their contributions to the care of their clients (Jones et al., 2015).

Preceptorship is fundamental to NPs' experiential learning, both before and after graduation and vital for NPs' successful transition to practice (Brown \& Olshansky, 1997; Jones et al., 2015; Leggat, Balding, \& Schiftan, 2015; Wiseman, 2013), the literature on preceptorship and transition is scarce (Jarrell, 2016; Poronsky, 2012). An NPs' role transition should be 
supported through a preceptorship. However, there are many obstacles to having such transition support, both before and after graduation.

Prior to graduation, NP students experience many issues with their preparedness for clinical experiences as well as with the quality and availability of their preceptors and clinical sites. Many preceptors have expressed concern about students' preparation level and questioned their preparation for practice (Roberts et al., 2017). NP students spend many hours at their clinical sites to learn their skills (Flott \& Linden, 2016). The challenges of obtaining clinical sites and preceptors are well documented (Drayton-Brooks, Gray, Turner, \& Newland, 2017). Additionally, the literature showed that evaluating NPs' competence based on the number of clinical hours may be insufficient (LeFlore, \& Thomas, 2016): Many NP students may complete the required clinical hours but never develop some important competencies that are necessary for skill acquisition. Quality clinical education is imperative for NPs' successful transition to practice.

To compensate for the lack of clinical sites, graduate nursing programs' leaders considered maximizing simulation laboratories and expanding the geographic areas for site searches (Erikson et al.,2014) as well as creating partnerships between academia and clinical practices (Sroczynski, \& Dunphy, 2012). While the creation of academia practice agreements, reaching out to rural clinics, and enhancing simulation learning are important, the attempts were inconsistent and school specific. Lack of preceptors and clinical sites could cause students and faculty to settle for less suitable learning environments. 
Further, preceptors are struggling to accommodate NP students' learning needs due to preceptors' needs to complete their duties of caring for complex patients within the strict time constraints of their schedules (Giddens et al., 2014; Keough et al., 2015; Poronsky, 2012) and given the increased demands of billing-related documentation (Wiseman, 2013). Most practices are busy and patient care takes priority over NP training (Bazzell \& Dains, 2017; Roberts et al., 2017). Adding additional responsibility to preceptors' already busy day without any adjustment could cause a decrease in a preceptor's productivity (Webb et al., 2015). Without allocating appropriate time to train, it is not possible to provide NP students with clinical education.

Other barriers to proper training include space limitations, inappropriate settings, patient volume, student's limited EMR access, and preceptors' lack of training (Roberts et al., 2017). Though $36.7 \%$ of preceptors expressed desire to be taught to train (Roberts et al., 2017) and preceptors play a key role in NPs' transition, few organizations provide any preceptor training to ensure that NP students and new NPs are supported in their learning of a new role (Bazzell \& Dains, 2017). Among graduate nursing preceptors, $47.3 \%$ reported no preparatory work to help them to train students (Donley et al., 2014). However, structured preceptorships have a positive effect on NPs' clinical reasoning (Bazzell \& Dains, 2017). Without knowing how to precept, preceptorship becomes a variable and unreliable experience.

Lack of preceptors' compensation is yet another barrier to a successful preceptorship. Most preceptors reported never having any monetary compensation (Wiseman, 2013). Financial compensation of clinical preceptors is minimal, with only $4 \%$ of NP programs offering this type of compensation (AACN, 2015; Erikson et al., 2014). However, “payment incentives for 
community-based sites were used by $71 \%$ of doctor of osteopathy (D.O.), $20 \%$ of physician assistant (P.A.), 15\% of medical doctor (M.D.)" (Erikson et al., 2014, p.3). Without proper compensation for preceptor's work, preceptor fatigue is likely to occur; preceptors may choose to stop training (Forsberg, Swartwout, Murphy, Danko, \& Delaney, 2015). Lack of compensation may affect the quality of NP students' clinical experiences.

After NPs graduate, NPs' support, if offered, may include collaborative physicians, assigned or formal preceptors, informal preceptors, on-the-job training, or post-graduation residency programs. Many medical professionals are being supported through the well-structured clinical education, both before and after graduation. There is a gap in how the transition to practice is regarded by the nurse practitioner profession and other medical professions. With about $90 \%$ of NPs expressing an interest in post-graduation residency programs in 2016 (Hart \& Bowen, 2016), only 37 NP primary care post-graduation training programs were available across the United States. They graduated approximately 150 NPs annually (M. Flinter, personal communication, June 8, 2016). With 23,000 NPs graduating in the years of 2015-2016 (AANP, 2017a) and 37 NP post-graduation programs available (M. Flinter, personal communication, June $8,2016)$, there was only one such program per 621 graduates. Thus, with less than $0.7 \%$ of NP graduates supported through the residency programs, many remain unsupported.

Other resources for novice NPs are also limited. Formal NP preceptorship is uncommon (Wilkes \& Feldman, 2017) with only $17 \%$ of novice NPs reporting having a formal preceptor during their role transition at work (Hart \& Bowen, 2016). With the lack of formal preceptorship to support novice NPs' role transition after graduation, the major source of preceptorship for NPs 
comes through a formal collaborative relationship with their assigned physicians; thus, most NPs' preceptors are MDs (Jones et al., 2015). Most novice NPs must have a collaborative agreement with MDs and rely on MDs' support; however, MDs' abilities to train may be limited. Medical and nursing paradigms differ significantly and yet, MDs are expected to guide NPs in their role transition. Misunderstandings about the NP role and scope of practice appear to be a barrier to their role acceptance (Lipley, 2014). Physicians' knowledge of NPs' role and scope of practice is important for these professionals' effective collaboration. However, MDs have little familiarity with NPs' role, education, and training (Schadewaldt, McInnes, Hiller, \& Gardner, 2013; Van der Biezen, Derckx, Wensing, \& Laurant, 2017), which is the most common barrier to a successful NP and MD collaboration (Schadewaldt et al., 2013). Besides not being familiar with NPs' role, novice NP collaborators often combine the responsibilities of providing NPs with advice along with the simultaneous evaluation of NPs' job performance (Poronsky, 2012). Thus, MDs are often NPs' employers, and they expect NPs to bring profit to their practices. Therefore, instead of having novice NPs' successful transition at heart, MDs' priority often becomes the profit NPs bring to the practices. However, this collaborative relationship may be the only source of preceptorship support a novice NP has after graduation.

Some scholars have explored the concept of transition and preceptorship. Several studies were conducted to learn about NPs' role transition after graduation (Brown \& Olshansky, 1997; Chappell, 2014; Flinter \& Hart, 2016). However, the role transition process starts long before the graduation (Heitz, Steiner, \& Burman, 2004; Steiner et al., 2008). Recent studies have focused on preceptors' incentives and post-graduation residency programs. Scholars confirmed that NPs' 
role transition and preceptorship are in great need of support. While post-graduation residency programs seem to be an excellent solution to an issue of a difficult role transition, they are rarely available to new graduates (AANP, 2017a; M. Flinter, personal communication, June 8, 2016). The entire role transition process, which includes periods before and after graduation, remains a complex phenomenon that needs to be explored. How this process is supported through preceptorship also remains largely unknown. While various general aspects of the postgraduation transition process have been outlined in other studies, focusing this study on transition's support through preceptorship uncovered new elements of NPs' role transition that can be further supported. Policies supporting NPs' transition through a preceptorship have yet to be developed.

\section{Problem Statement}

The problem is that NPs' role transition is difficult and poorly supported through outdated preceptorship model. The number of NPs grew by 100,000 since 2009 (AANP, 2017a) in response to recent increase in demand for NPs (Giddens et al., 2014) due to the projected shortage of 40,000 PCPs by 2025 (Sroczynski \& Dunphy, 2012). The number of NP educational programs also grew to compensate for the increased need for NPs (AANP, 2017b). Though the number of NPs is increasing, employee turnover rate for NPs is double of that of MDs (Fitzpatrick \& Gripshover, 2016). Having to care for complex clients may cause novice NPs to doubt their skills (Graue, Rasmussen, Iversen, \& Dunning, 2015). In fact, according to Hart and Bowen (2016), 47.7\% of NPs felt that they were practicing outside their scope of practice during their first year of practice. 
In previous research, scholars indicated that the current clinical training model is too outdated (AACN, 2015; Giddens et al., 2014; LeFlore, \& Thomas, 2016) to meet the current health care demands for complex patient care (Giddens et al., 2014). With NP role transition lasting to up to 2 years after graduation (Heitz et al., 2004), the NPs' role transition after graduation is also ill-supported. Formal preceptorship is uncommon (Wilkes \& Feldman, 2017) with only $17 \%$ of novice NPs reporting having a formal preceptor during their role transition at work (Hart \& Bowen, 2016) and less than $0.7 \%$ of NP graduates are supported through residency programs (AANP, 2017a; M. Flinter, personal communication, June 8, 2016).

The RN to NP role transition has been identified as difficult and stressful (Brown \& Olshansky, 1997; Flinter \& Hart, 2016; Steiner et al., 2008). Role transition is a complex process that requires substantial support (MacLellan, Levett-Jones, \& Higgins, 2015). Limited research is available on the NP transition- to- practice phenomenon (Faraz, 2016). Knowledge is also scarce on identifying the important elements of the NP role transition process, both before and after graduation. Role transition is a complex and multistage process that is difficult to encompass in one study; however, focusing the study on role transition in relation to preceptorship was a logical approach to investigating this multifaceted phenomenon. Because in previous research, scholars identified lack of preceptorship, both before and after graduation, examining the elements of role transition together with preceptorship helped close the gap in knowledge and thus helped employers and NP training programs develop interventions to improve NPs' transition to practice. 


\section{Purpose of the Study}

The purpose of this hermeneutic phenomenological study was to understand and describe the meaning of NPs' role transition experiences while in a preceptorship before and after graduation. The central phenomenon of interest in this study was NPs' role transition while under a preceptor's guidance. The purpose of a phenomenological study is not to simply document the description of an everyday experience related to the studied phenomenon; rather, it is to capture the uniqueness or the meaning of such an experience so that those who interact with the phenomenon have additional insights about it (Van Manen, 2017). Thus, in this dissertation, I gained an in-depth understanding of NPs' experiences with their role transition in relation to preceptorship support.

\section{Research Question}

The research question posed in this study was as follows: What was the experience of transition like for an RN becoming a NP under the guidance of a preceptor during her or his academic program and after graduation?

A simple lack of knowledge about the phenomenon is not the only indication for conducting a phenomenological study; on the contrary, there may be a lot of information available about the phenomenon, but well-formed assumptions may prevent people see the essences of the phenomenon (Van Manen, 1990). Approaching the phenomenon again allows the phenomenon to transpire without imposing these assumptions, which may point toward different essences of that phenomenon (Van Manen, 1990). Thus, I formulated my research question in a 
way that is consistent with the phenomenological approach to obtain descriptive pre-reflective data without imposing mine or my participants' assumptions.

\section{Theoretical Foundation}

The theoretical lens of this study consisted of the combination of two theories, Schlossberg's transition theory, and Collins, Brown, and Holum's (1991) Cognitive Apprenticeship Model (CAM). The concept of transition was guided by transition theory, and the concept of preceptorship was guided by CAM. Schlossberg's theory originated in the field of psychology from her earlier model of analyzing human adaptation to transition (Schlossberg, 1981) to help develop coping strategies for adults in various transitions. The assumption behind Schlossberg's transition theory is that the type and stage of one's transition, as well as one's available resources and coping mechanisms, can facilitate or inhibit successful transition outcomes (Anderson, Goodman, \& Schlossberg, 2011; Schlossberg, 2011). Schlossberg's theory has been widely applied to role transitions and college students' transitions (Chickering \& Schlossberg, 1995).

To guide the exploration of preceptorship, the CAM was used. Apprenticeship or learning from a master in a social environment within the context of one's profession is the concept used for both teaching and learning through practice (Dennen \& Burner, 2008). The CAM's concepts were used to guide the exploration of NPs' experiences with preceptorships during their role transition process. While the transition theory was helpful in describing and understanding the process of RN to NP role transition, the CAM enhanced the study's focus on the context of 
learning from a master, in which knowledge is transferred (Dennen \& Burner, 2008). Chapter 2 addresses transition theory and CAM in greater detail.

\section{Nature of the Study}

The nature of this study was qualitative. The approach to my qualitative inquiry was hermeneutic phenomenology. Qualitative studies allow one to choose "depth" over "breadth," to explore the phenomenon of interest (Patton, 2015). Because the NPs' role transition and its support through the preceptorship is under-researched, thick description added to better understanding of various factors related to preceptorship that influenced NPs' transition to practice process.

Van Manen's (1990) approach to hermeneutic design and analysis was used to guide this dissertation study. Van Manen (1990) stated that both description and interpretation of hermeneutic phenomenology is intimately connected to the situation or the context. Though considering pure description guided by Husserl is important to uncover the important attributes of the studied phenomena, exploring the contextual attributes of participants' experiences is also significant for the study's practical application. Husserl's tendency to objectify the phenomenology method may cause to ignore the content in which phenomenon occurs (Van Manen, 1990). The hermeneutic approach assists in capturing the meaningful interpretation of the experience allowing for grasping its significance (Van Manen, 1990). Thus, in this study, I used both descriptive and interpretive approaches to connect the concept of role transition to the transition's context related to preceptorship. When used together, the descriptive and interpretive approaches allow a deeper understanding of the participants' experiences (Matua \& Van Der 
Wal, 2015). Capturing description of experiences along with the context made the study results more applicable to various contexts and situations where processes need to be improved. Additionally, a hermeneutic phenomenological approach assisted with the identification of common meaning of the transition process from several NPs, possibly leading to the future creation of new policies and approaches to foster efficient and effective transitions.

Data were collected through face-to-face and web-assisted interviews of NPs who were licensed and board certified. Additional inclusion criteria were as follows: Current or former practice as an NP in the United States, having a collaborative agreement with physician(s), completion of an orientation period specified by an employer, practicing for at least 3 months following an employer's orientation, and practicing for no longer than 5 years after graduation.

\section{Definitions}

\section{Central Phenomenon}

The central phenomenon in this study was role transition supported through preceptorship. Role transition is a stressful change process that includes changes in NPs' assumptions and behaviors (Schlossberg, 1981). Transition support through preceptorship is defined as experiential learning through cognitive apprenticeship (Collins et al., 1991).

\section{Key Concepts}

Apprenticeship: Apprenticeship or learning from a master in a social environment within the context of one's profession (Benner, Sutphen, Leonard-Kahn, \& Day, 2008; Dennen \& Burner, 2008). 
Cognitive apprenticeship: In a cognitive transfer of knowledge master ensures apprentices' understanding of the process of a given skill, adjusting apprentices' experiences to their theoretical knowledge to ensure the relevance of their experiences, and encouraging the apprentices' ability to transfer common tasks across the different contexts (Collins et al., 1991). Collaborative NP-MD agreement: The collaborative agreement defines MDs and NPs' relationship and their legal requirements (Herman \& Ziel, 1999).

Essence: Essence is a textual description of a phenomenon that reveals the structure of that phenomenon in a way it was not seen before, allowing for a deeper understanding of the importance of the experience (Van Manen, 1990).

Mentorship: Mentoring or mentorship is defined as a relationship between the two people, mentor, who is more experienced and mentee who is less experienced; the goal of such relationship is mentee's growth (Green \& Jackson, 2014).

NP-MD collaboration: The collegial relationship between NPs and MDs that requires three to six months to develop and is characterized by frequent lack of MDs' knowledge regarding NPs' role and scope of practice; both professionals' sharing of the workload; effects of the financial constraints and reimbursement on the collaboration; the degree of NPs' autonomy and supervision by MDs; as well as the degree of hierarchical relationship between NPs and MDs (Schadewaldt et al, 2013).

Post-graduation residency programs: Intensive, highly structured postgraduate NP training programs that provide a "safety-net" learning environment for novice NPs to develop mastery of their new role (Flinter \& Hart, 2016). 
Preceptorship: Preceptorship was defined as a guiding and supportive one-on-one relationship between the trainee and the preceptor, both before (Kelly \& Mcallister, 2013) and after graduation (Korzon \& Trimmer, 2015). Preceptorship is often a short-term arrangement (Nielsen et al., 2017).

Transition: A transition occurs when a person changes the assumption about self and the world along with corresponding changes in behaviors and relations (Schlossberg, 1981).

Reduction and epoche: Epoche and reduction are related to phenomenological analysis. Epoche means suspending and eliminating bias to gain a fresh look at the phenomenon (Patton, 2015). The reduction is performed through bracketing of the existing knowledge and literature to reflect on the phenomenon of interest (Patton, 2015).

Role change: role loss (e.g., loss of an $\mathrm{RN}$ role) or role gain (e.g., a new role of an NP) that is accompanied by stress and conflicting negative and positive feelings (Schlossberg, 1981).

\section{Assumptions}

One assumption was that new NPs desire a smooth and meaningful transition from school to practice. Theoretical assumptions included the two chosen theories' assumptions. First, Schlossberg transition theory's assumption is that the type and the stage of one's transition along with the available transition resources and one's coping mechanisms can facilitate or inhibit one's transition (Anderson et al., 2011; Schlossberg, 2011). Next, the CAM's assumption is that quality of preceptorship depends on trainees' baseline knowledge, preceptor's teaching strategies, sequencing of instruction, learning environment, and quality of post-graduation 
training programs and collaboration (Collins et al., 1991). The above assumptions were essential to support the data collection and analysis.

\section{Scope and Delimitations}

The scope of this study was limited to the participants' descriptions of their experiences and journals; the study did not include direct observations or videotaping.

All participants graduated from an NP program; however, those who graduated from a BSN to DNP track were excluded from the study due to the differences between the master's and doctoral programs. Additionally, only participants who graduated less than 5 years ago were included in the study to facilitate recall of the role transition experiences. Those NPs who never practiced as NPs or those NPs who practiced for less than 3 months after the completion of the employer's orientation program were also excluded because they have not experienced the second post-graduation stage of NP role transition. Further, those NPs who completed the postgraduation residency program were excluded from the study because they represented less than $1 \%$ of all the NP population in the United States, and including them could have distorted the understanding of the phenomenon of interest. The results from this study informed those who are involved in NP education and practice. Additional research must be conducted to test the findings of this study for other APRNs' role transitions before any data transferability could be entertained.

\section{Limitations}

While I used sound strategies to produce quality results, my study had some limitations. One limitation was related to the accuracy of my data in that it depended on my participants' 
recall. The validity of qualitative research is dependent on the quality of the information that the participants provide about the research question (Patton, 2015). To increase validity or credibility of the data, I asked my participants prompt questions during the interviews as well as directed them to describe their experiences with examples as opposed to opinions. I encouraged them to provide thick descriptions. I used interview data and field notes to triangulate data. Furthermore, I used debriefing along with follow-up phone calls, as needed, after each interview to ensure the accuracy of the data.

It was important to clarify potential biases, which could distort the reliability of the data. To avoid this, I used reflexivity in describing my role as a researcher to show how my personal experience and literature review could affect my results.

Because I was the only investigator in this study, I was not able to perform member checking or crosscheck coding, which is advisable with a large amount of data in qualitative studies. To increase the dependability of the data, I transcribed the data myself and doublechecked it for any errors and against my codebook. While it may be possible to generalize the results of this study within the same population, transferring the results outside the study setting and the population was not possible unless additional studies were conducted. In qualitative research, internal generalization is possible; however, the transferability is problematic (Maxwell, 2013).

Overall, the main limitation of a qualitative study related to that it had large amount of data, which was hard to manage. A large amount of data may overwhelm a new researcher. I attempted to limit the amount of data in my study to make it manageable using the interview 
protocol and incorporating theories to provide for a theoretical lens to focus it. Though the interview protocol helped me to limit the data to relevant information by focusing the interview, it also allowed me to remember to cover all required study areas consistently across all participants. While I took care of prestructuring my study to make it feasible and high quality, I also stayed open to the new emergent data.

\section{Significance of the Study}

NPs' role transition is a complex process that starts with an RN entering NP school and ends years after graduation; NPs' transition to practice has experiential learning at its core. Understanding how NPs' role transition is supported through preceptorship will guide future efforts directed at improving transition support for NPs. In this study, I addressed the gap in literature related to NPs' role transition and its support through preceptorship. Strategies to facilitate NPs' role transition can be developed and translated into practice based on this study's results. Positive social change can be affected by this study through the dissemination of this study's findings among the NP educators, NP employers, and NPs themselves.

\section{Significance to Practice}

One of the aims of this study was to produce findings that could fill the knowledge gap and be applied to practice by creating new strategies and policies to support NPs' role transition. This study was unique because it addressed the under researched area of advanced nursing practice and education (Poronsky, 2013) in a growing population of NPs. Many NPs struggle with their clinical skill level preparation (Logan et al., 2015), which may contribute to NPs' stressful role transition and high turnover rate. Adequate clinical preparation is essential for NPs' 
successful transition to practice (Clabo et al., 2015). The insights of this study will inform professionals on what areas can be better supported to facilitate NPs' role transition.

\section{Significance to Theory}

The contribution of this study's findings to knowledge and nursing discipline can be appreciated by realizing the significance of role transition support in the nursing profession. More specifically, I aimed to address NPs' role transition supported through preceptorship. This study's findings may prompt further research in this direction, linking my findings to future research. Filling the gap in the existing literature, along with uncovering new elements of NPs' role transition and its support, may help raise awareness of NPs' experiences with the role transition; it may help create a new scholarly dialogue that can lead to improved transition practices. Because research is limited in the area of NP transition and preceptorship, this study's findings may prompt other scholars to further explore this important phenomenon, which could lead to further improvements within the NP profession.

\section{Significance to Social Change}

Social change is "the alteration, adaptation, modification, or transformation of social structures, social institutions, socio-cultural characteristics, social relations, or socio-political ideology" (O'Leary, 2007, p. 249). The shortage of available PC providers, combined with growth in the elderly population, and an increased need for NPs, made it necessary to explore the factors that influence NP role transition (Barnes, 2015a; Chattopadhyay et al., 2015). This study's findings have potential implications for positive social change for the NP students, NP faculty members, NP academic programs, clinical preceptors, patients, clinical practices, and 
interdisciplinary relationships in communities. This study's findings may offer useful insight on how to restructure preceptorships to facilitate NPs' successful transition to practice. Improving NPs' role transition may lead to an increased retention rate of NPs, improved job satisfaction, and competence.

\section{Summary}

The number of NPs continues to increase in order to satisfy the demand for quality and affordable health care; however, NP programs and employers have failed to adapt to this growing demand and support the transition of NPs into their new role. NPs' responsibilities have grown with the changing demands of health care to accommodate complex, diverse, and underserved patient populations. However, many NP graduates lack important advanced skills; struggle to close the gap between the outdated clinical education model and the growing demands of current practice environments. Despite the increased need for NP providers, there is a limited understanding of NPs' experiences with their role transition before and after graduation. While the literature pointed toward the insufficient transition support through the preceptorship, both before and after graduation, there was a paucity of knowledge on how much NPs' role transition is supported by preceptorship. Gaining insight into NPs' experiences with their role transition and how it was supported by conducting a hermeneutic phenomenological study allowed the voices of NPs to be heard and new knowledge to be gained.

Chapter 2 includes a review of the literature on study's main concepts of role transition and preceptorship. 
Chapter 2: Literature Review

\section{Introduction}

The RN to NP role transition has been identified as difficult and stressful (Brown \& Olshansky, 1997; Flinter \& Hart, 2016; Steiner et al., 2008). NPs' challenging transition to practice may be related to the gap between education and practice (Fitzpatrick \& Gripshover, 2016). The NPs' clinical training is ill-supported before (American Association of Colleges of Nursing (AACN), 2015; Donley et al., 2014; Logan et al., 2015; Roberts et al., 2017; Webb et al., 2015) and after graduation (Brown \& Olshansky, 1997; Flinter \& Hart, 2016; Steiner et al., 2008); thus, NPs' clinical training lags behind of the demands of current health care system (Hallas, Biesecker, Brennan, Newland, \& Haber, 2012; Sullivan-Bentz et al., 2010). The clinical training model, “one-to-one preceptor-student model, designed to serve a much more limited population of students" has not changed in 45 years and is outdated and ineffective (AACN, 2015, p. 1; Giddens et al., 2014; LeFlore \& Thomas, 2016). NPs learn their clinical skills from a preceptor through the process of preceptorship, which is vital for the NPs' transition to practice (Brown \& Olshansky, 1997; Jones et al., 2015; Leggat et al., 2015). However, the literature on preceptorship is scarce for NP students or NP graduates (Jarrell, 2016; Poronsky, 2012).

Similarly, there is limited research on the role transition of NPs (Faraz, 2016). Furthermore, no current studies were found on NPs' experiences with preceptorship during both transition stages, before and after graduation.

In Chapter 2 I present the literature on NP role transition and preceptorship beginning with discussion of NP role, which includes limited historical data on role development, a 
description of NPs' education and licensure, and information about the requirements of an NPMD collaborative agreement. The discussion of the NP role is followed by a discussion of the NP role transition, and a description of the transition stages. Rich description of the NP role allows for a deeper understanding of the NPs' role transition process. Next is a description of the preceptorship concept with respect to the NP role. Finally, a more in-depth description of the NP transition characteristics is connected to the description of NP transition resources, and the preceptorship resources available to NPs before and after graduation.

\section{Literature Search Strategy}

For this literature review, I searched the following databases until saturation was achieved: Academic Search Complete, ProQuest Nursing \& Allied Health Source, CINAHL Plus with Full Text, MEDLINE with Full Text, ProQuest Health and Medical Collection, Web of Science, OVID, PubMed, Education Source, ERIC, Science Direct, Sage Journals, PsycINFO, and PsycARTICLES. The search was limited to the years 2010 through 2017. The following search terms were used: graduate nurse, advanced nursing, nurse practitioner, APRN, masters prepared, advanced nurse, nursing student, practicum, residency programs, residencies, school, program, clinical, clinical education, nursing education, health education, training, clinical site, student, preceptor, preceptorship, apprenticeship, mentor, mentorship, transition, role transition, role change, nurse's role, primary care, qualitative research, collaboration, and clinical competence. Studies related to undergraduate and doctoral nursing programs were excluded. 


\section{Theoretical Foundation}

The way researchers use existing data before their actual data collection is what makes theory use in qualitative study unique. While it is imperative to have an "open mind" when conducting qualitative research, it is also important to know and build on the previous knowledge to avoid having an "empty head" (Strauss \& Corbin, as cited in Kenny \& Fourie, 2015). In qualitative research, one may choose to explore what is already known about a phenomenon, using the theory that reflects that knowledge at the beginning of his or her study to guide the research process (Tavallaei \& Talib, 2010). Thus, in my study, I reviewed the existing literature and applied some concepts from the two theoretical frameworks to loosely focus my study. My focus was not restricted by the chosen theoretical concepts, but rather they helped illuminate the areas I was researching, to make my study feasible and effective. More specifically, knowledge obtained from my review of previous related theories and research helped me to focus my inquiry about NP role transition and preceptorship.

\section{Theoretical Framework}

To ensure that my study's initial concepts were grounded in existing knowledge, I have chosen Schlossberg's transition theory (1981), and Collins et al.'s (1991) CAM. The combination of concepts from the two chosen theories sensitized me to the most essential elements within my main broad concepts of preceptorship and role transition. More specifically, the Schlossberg transition theory directed me to examine the stages of NPs' transition process as well as explore various factors that may affect NPs' transition to practice during these stages. Further, the CAM model was helpful in my examination of major elements of NPs' 
preceptorship (Collins et al., 1991). Examining the cognitive strategies used in experiential learning is a foundation of CAM model; it combines schooling or an invisible process of thinking with the apprenticeship (Collins et al., 1991). While these well-established and wellsupported theories anchored my study's concepts to guide my study's assumptions (Baxter \& Jack, 2008, p.553), I stayed open to the emerging data to avoid any distortions and limitations that can be potentially caused by using any of the preexisting knowledge. (Maxwell, 2013). While no one theory offers the explanation that fits my study completely, using the combination of theories assisted in my understanding of processes that have been researched so far. To understand how each theory illuminated my study's concepts, each theory was reviewed in relation to my study.

Transition. Schlossberg's theory has been widely applied to the transition of college students. Schlossberg's theory has originated in the field of psychology from Schlossberg's earlier model of analyzing human adaptation to transition (Schlossberg, 1981) to help develop coping strategies for adults in various transitions. Bridges' transition model of three stages, ending, neutral zone, and beginnings also served as a foundation for Schlossberg's transition model (Porosky, 2013). Thus, separating from the previous role to gain a new identity becomes an important part of the role transition process (Poronsky, 2013). The neutral zone stage is a stressful period of creating and living with the new routines (Poronsky, 2013). Schlossberg's transition theory's assumption is that the type and the stage of one's transition along with the available resources and one's coping mechanisms can facilitate or inhibit one's transition 
(Anderson, et al., 2011; Schlossberg, 2011). Schlossberg's transition theory helped understand the transition and how one copes with the transition stressors.

Because a person's transitions, challenges and coping strategies may vary significantly, it is important to approach one's experience with transition holistically. Psychologists and counselors used transition theory to help individuals cope with life transitions (Poronsky, 2013). According to the transition theory, the individual's resources used to cope with the transition relate to the four S's, “the situation itself, the individual's personal attributes (self), available support, and the individual's strategies for coping" (Schlossberg, 2011, p. 160-161). The four S's define how one will handle the transition, regardless of its type or stage (Goodman, Schlossberg, \& Anderson, 2006). Examining resources NPs utilize to cope with transition challenges was essential in understanding the role preceptorship has in the NPs' transition process.

The four S's allowed for a holistic approach when examining NPs transition' challenges and resources. The first S, situation, includes factors that triggered one's transition, transition timing in terms of the person's context, the degree of control one has over the transition, the predicted duration of the transition process, one's prior experience with similar transitions, concurrent stressors, and the individual's awareness of the transition process (Goodman et al., 2006; Poronsky, 2013). The second S, self, includes factors related to the person's unique characteristics such as demographics and coping mechanisms an individual usually uses to deal with life challenges (Goodman et al., 2006). The third S, support, includes the types of support such as relationships and networks; functions of support such as feedback and assistance; and measurement of support, which includes recognizing the specific resources that are available for 
an individual (Goodman et al., 2006). The fourth S includes strategies people use to cope with the stressors of transition, which may include seeking information, person's actions, as well as strategies one uses to continue participating in the experience, such as denial (Goodman et al., 2006). Exploring resources NPs use to cope with their transition stressors may help in future development of strategies to strengthen NPs' transition process. Schlossberg's theory was helpful in examining NPs transition' context, potential resources, and transition stages. Therefore, it was important to explore NPs' transition experiences during the stages of moving in, moving through, moving out, and moving in again (Anderson, et al., 2011). Depending on what stage the NP is describing, his or her transition and perception of preceptorship may differ.

Experiential learning. While the transition theory was helpful in describing and understanding the process of RN to NP role transition, the cognitive apprenticeship model enhanced the study's focus on the context of learning from a master, in which the knowledge is transferred (Dennen \& Burner, 2008). To guide the exploration of preceptorship from the NPs' perspective, the CAM model was utilized. Different terms, including mentorship, preceptorship, and apprenticeship, have been used in the literature to describe various learning approaches. Exploring various terminologies related to experiential learning assists in a deeper understanding of how knowledge is transferred in the clinical context. In the next sections, I describe each term.

Mentorship. Mentoring is defined as a relationship between the two people, a mentor, who is more experienced and a mentee who is less experienced; the goal of such relationship is the mentee's growth (Green \& Jackson, 2014). Mentorship and mentor are close terms; according to the Merriam-Webster dictionary (2017) mentor is defined as "someone who teaches 
or gives help and advice to a less experienced and often younger person" (para 1); to mentor is to "teach or give advice or guidance to a less experienced person" (para 2). The term mentorship is often used in literature to describe the relationship between NPs and their clinical teachers or experts, both before and after graduation. The concept of mentorship or mentoring has been long used to describe the relationship between the mentor and the mentee. In fact, professionals began using the concept of mentorship or mentoring applied to career in mid-1970's (Meier, 2013). A mentor is someone who assists the novice professional to advance in their career (Meier, 2013). Thus, the definition of mentorship is complex.

Though the term mentorship is sometimes used interchangeably with apprenticeship and preceptorship, its meaning is slightly different. While the main mentor's responsibility is to provide training, mentors also ensure closer interaction with the mentee by providing other types of support (Wilkes \& Feldman, 2017). Mentorship is usually regarded as a long-term relationship, which is less commonly seen in today's busy learning environment; it goes beyond the scheduled activities (Wilkes \& Feldman, 2017). In current clinical learning environments, it is usually difficult to create and maintain mentorship because of a mentor's work demands, change of jobs, role conflict between being a mentor and an employee, and competition with other professions (Wilkes \& Feldman, 2017). Mentorship is generally a voluntary (Roberts et al., 2017) long-term relationship that includes both professional and personal growth outcomes of a mentee as well as growth and professional satisfaction for the mentor (Nielsen et al., 2017). Because NPs' relationships with their preceptors are usually short-lived and do not extend to include personal growth, the term mentorship, though frequently used in literature, may not be a 
good fit to describe this type of relationship. However, a thorough understanding of this term may be useful in distinguishing it from other terms.

Though uncommon, mentorship is significant to mentees' successful role transition. Mentorship is vital for NPs' transition to practice (Brown \& Olshansky, 1997; Jones et al., 2015; Leggat et al., 2015). Mentorship programs are necessary for NPs' professional development (Jarrell, 2016). Formal and informal mentorships exist (Meier, 2013). In programs that utilize formal mentoring, organizations are responsible for training mentor and mentee, setting goals and schedules, as well as providing evaluations (Meier, 2013). However, such mentoring is rarely available to novice NPs, though it is necessary for NPs to successfully transition to their new role and retain their job. In fact, employee turnover rate for NPs is doubled that of MDs (Fitzpatrick \& Gripshover, 2016). Mentorship programs facilitate novices' socialization into the profession, assist with successful role transition, and improve retention rates (Meier, 2013). In the absence of formal mentoring, some novice NPs obtain informal mentors. Informal mentoring is usually initiated by the mentee (Meier, 2013). Both formal and informal mentorship can improve NPs' role transition.

To be successful mentorship must include several necessary attributes. Namely, mentorship must include commitment (Fawcett, 2002 as cited in Horner, 2017), objectives, expectations, and boundaries (Horner, 2017); better outcomes of formal mentorship were reported when mentors and mentees were prepared for the mentorship (Poronsky, 2012). The attributes of mentorship or mentoring also include nurturing, mentoring roles, role development, and caring relationship (Meier, 2013). Nurturing serves to make a mentee feel emotionally safe 
to learn new skills; mentor's roles among others include coaching and role modeling. Role development includes presenting the mentee with opportunities for professional growth, and a caring relationship is a relationship in which mentor has the mentee's best interest at heart (Meier, 2013). Mentor-mentee relationship is based on trust and means devoting the time to help the mentee to grow professionally (Meier, 2013). For the mentorship to take place there must be a caring and open relationship between the mentor and the mentee; both must be committed to devoting time to this relationship (Meier, 2013). Thus, mentorship includes a learner-centered safe learning environment that fosters mentee's professional growth. Role transition can be improved by skilled mentorship.

Though mentorship is an effective way of supporting one's transition to practice, little is known about mentorship when applied to NP population. The literature on mentorship is scarce for the population of NP students or NP graduates (Jarrell, 2016; Poronsky, 2012). The majority of the research on mentorship in nursing has been conducted in the area of undergraduate nursing; however, gaining knowledge about NPs' experience with mentorship may assist in improving the quality of mentoring in current graduate nursing education (Nyhagen \& Strøm, 2016). More research is needed on the role of mentorship in NPs' transition.

Apprenticeship. The concept of cognitive apprenticeship is the central concept of a CAM model used in this study. This term definition is similar to the terms of preceptorship and mentorship. As per Merriam-Webster dictionary (2017), an apprentice is the "one who is learning by practical experience under skilled workers a trade, art, or calling;" it also means "to bind to an apprenticeship by contract or indenture" (para 1,2). Apprenticeship or learning from a 
master in a social environment within the context of one's profession is the concept used for both teaching and learning through practice (Benner, et al., 2008; Dennen \& Burner, 2008). Apprenticeship is the process of professional development from the novice to an expert level under the direction of an expert (Downey, Dalidowicz, \& Mason, 2015). Transfer of skills happens through the apprenticeship or a relationship between the expert clinician and a trainee, student, or a novice professional; the term of apprenticeship is sometimes used interchangeably with mentoring (Wilkes \& Feldman, 2017). Apprenticeship, just like preceptorship and mentorship, refers to the training of a novice professional. Noticeably, the term apprenticeship was seen less frequently during this literature review; when encountered, it was mostly related to the theoretical frameworks of the reviewed studies. Important division exists in the literature between the traditional and cognitive apprenticeship.

In a traditional sense, apprenticeship refers to learning how to perform the task through observing and gradually gaining more independence (Collins et al., 1991). Thus, traditional apprenticeship includes role modeling, "scaffolding, fading, and coaching" (Collins et al., 1991, p. 2). By role modeling, a master shows the apprentice how to perform the task; thus, it involves observation (Collins et al., 1991). Scaffolding is ensured by providing the apprentice with the support; the degree of independence may vary based on apprentice's need for support, which is faded gradually (Collins et al., 1991). Coaching is done through supervision and feedback by a master (Collins et al., 1991). While learning through observation is important in performing a task correctly, connecting the knowledge of content with an apprentice's experience is also needed; it is accomplished through cognitive apprenticeship. 
Understanding the skill assists NPs in closing the gap between their experiences and what was learned in school. Though traditional apprenticeship explains how learning occurs through the observation, cognitive apprenticeship underlines the importance of master's and apprentice's thinking process' transparency (Collins et al., 1991). Cognitive apprenticeship takes place when an expert makes his or her thinking process transparent to a trainee (Lyons et al., 2017). Thus, in cognitive transfer of knowledge, the master ensures apprentices' understanding of the process of a given skill, adjusting apprentices' experiences to their theoretical knowledge to ensure the relevance of their experiences, and encourages the apprentice's ability to transfer common tasks across the different contexts (Collins et al., 1991). The cognitive apprenticeship model aligns with the training of complex skills processes (Gardiner \& Anderson, 2013). Because the clinical environment alone is not sufficient for one's learning of skill, an expert has to be able to align clinical situations with apprentices' didactic knowledge and theory (Gardiner \& Anderson, 2013). Thus, explaining the skill while connecting it to the students' theoretical knowledge with its application in different contexts constitutes cognitive apprenticeship.

Preceptorship. The term preceptorship is also frequently used in literature to describe the relationship between an NP and a preceptor. According to Merriam-Webster dictionary (2017), the preceptor is a "teacher or a tutor" who is a "practicing physician who gives personal instruction, training, and supervision to a medical student or young physician" (para 1,2). Preceptorship is defined as a guiding and supportive one-on-one relationship between the trainee and the expert, both before (Kelly \& Mcallister, 2013) and after graduation (Korzon \& Trimmer, 2015). While mentorship is usually a long-term relationship, preceptorship is often a short-term 
arrangement (Nielsen et al., 2017). Preceptorship is also an essential part of nurses' transition to practice (Korzon \& Trimmer, 2015). Preceptorship is normally encountered by an NP during the graduate school when obtaining a clinical experience.

The terms preceptorship and mentorship are used interchangeably, and the structures of these two types of relationships have many similarities. Chen, Rivera, Rotter, Green, and Kools (2016) defined three types of preceptorship; formal preceptor-trainee, formal multiple preceptors from a single site-trainee, and informal multiple preceptors-trainee preceptorships. Regardless of the structure, just like mentorship, preceptorship is used to train NPs to function in their new role. Preceptorship is a method to promote professional development of a person to ensure safe and effective patient care (Nielsen et al., 2017; Roberts et al., 2017). The function of preceptors is to guide NPs' learning in a clinical setting (Johnson, O'Brien, Emerson, \& Reed, 2017). Obstacles to effective preceptorship found in the literature were similar to those in mentorship and included lack of time and workload adjustments for those who function in a role of a preceptor (Korzon \& Trimmer, 2015). With preceptorship being a short-term relationship, it may be more difficult for a preceptor to transfer knowledge to an NP. Because the term preceptorship is used more frequently both in the literature and in practice, I used the terms preceptorship, preceptor, student, and trainee throughout the rest of my study to ensure clarity and consistency. In application to my proposed study, the CAM model guided the concept of cognitive preceptorship. It is important to note that CAM helped guide the understanding of a cognitive preceptorship as opposed to traditional preceptorship. CAM's concepts included those elements that both NPs and their preceptors must possess for an effective preceptorship to take place. 
Namely, these elements of the CAM model include a NPs' baseline knowledge necessary to function in a role of an NP, preceptor's teaching strategies, preceptor's ability to increase the complexity and diversity of NPs' clinical experiences, and learning environment, both before and after graduation (Collins et al., 1991).

To demonstrate my studied concepts, I created a concept map, which is depicted below in Figure 1. The sources used to create a concept map of a study may include researcher's experience, literature review, pilot studies, and model or concepts in an attempt to clarify studied concepts (Maxwell, 2013). My concept map is based on my literature review and the two chosen theories that will be used to bring my studied concepts to clear view. To reiterate, in this study, I used Schlossberg's concepts of transition stages and transition resources as well as Collins et al.'s CAM, as described above (see Figure 1). The attributes of the role transition concept itself were derived from the literature review (see Figure 1). These attributes assisted in describing the key elements related to NP role transition; these elements included learning of a new role; assuming new responsibilities and increasing autonomy; attempting to create a new role identity; and struggling with many negative emotions (Barnes, 2015b). While my concepts were borrowed from preexisting theories and literature, together they constructed a blueprint of my dissertation that adds transparency to my study. 

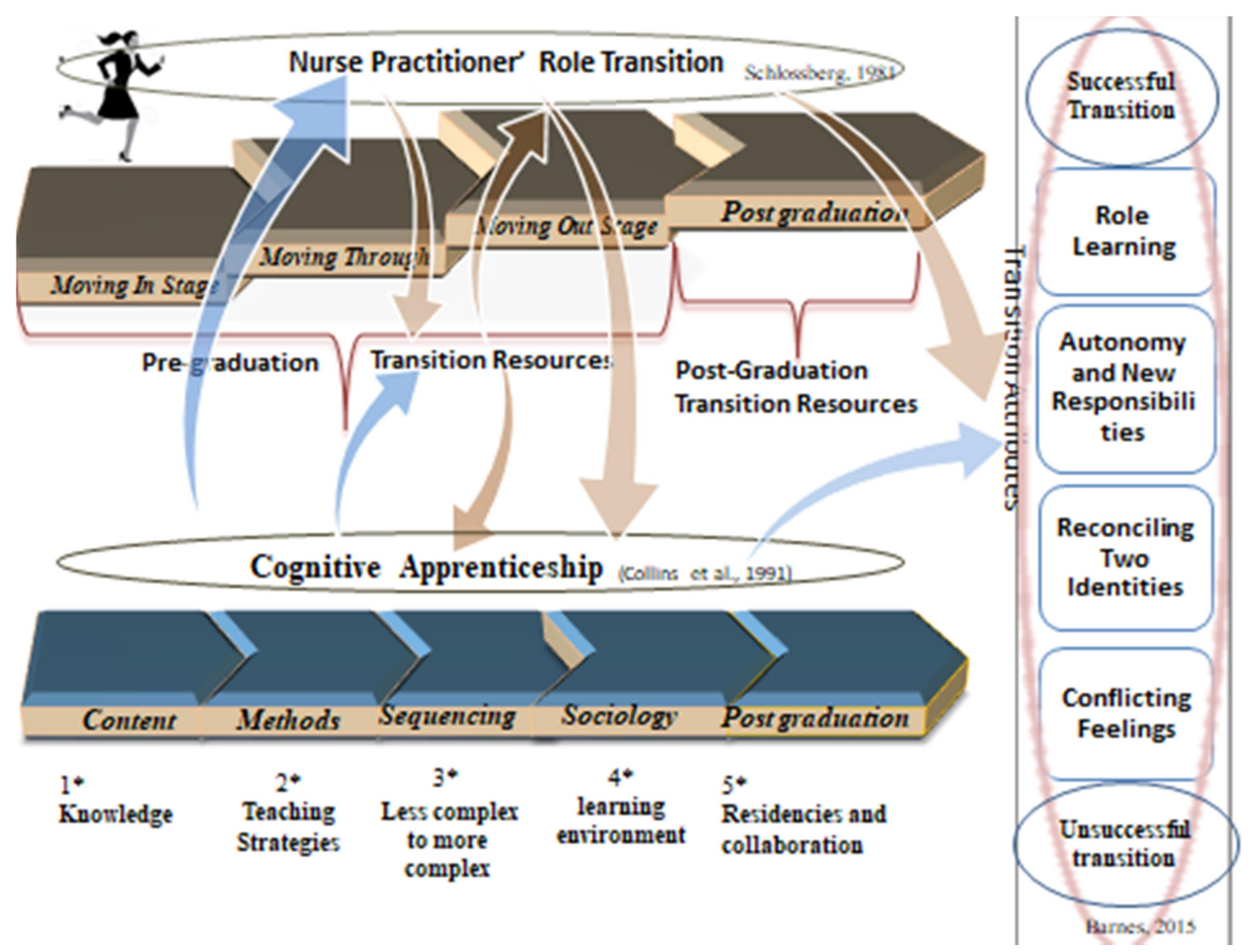

Figure 1. Role transition and cognitive mentorship concepts. *1- Content - necessary knowledge and learning strategies;*2-Methods Teaching strategies (Modeling, coaching, scaffolding, articulation, reflection, and exploration);*3-Sequencing (increasing complexity and diversity); *4-learning environment Leadership style (values quality care and trainee education; trainee familiarity with the site orientation; *5-Post-graduation mentorship (awareness of NP scope of practice, education, legal responsibilities; previous experience working with NPs, sharing responsibilities of complex patients, workload, communication, mutual respect, reciprocal collaboration (Collins et al., 1991; Schadewaldt et al., 2013).

Overall, in examining the role of a theory, it is important to realize that no chosen theory can provide a perfect fit to one's study; thus, it is important to be aware of the chosen theory limitations. Because qualitative research is fluid and context-dependent, I do not see the use of 
theory in my research as being fixed. Thus, though the transition theory and CAM model fit well with my research topic, I stayed open to the emergent data during my study.

\section{Nurse Practitioner Role}

APRN is a broad term that includes four types of advanced practice nurses. In the United States, these four types are as follows: (a) nurse practitioners (NP), (b) clinical nurse specialists (CNS), (c) nurse midwives, and (d) nurse anesthetists (Parker \& Hill, 2017). The APRN role was first introduced by Loretta Ford and pediatrician Henry Silver in 1965 at the University of Colorado to increase patient access to care due to the increased shortage of primary care physicians (PCPs; Hevesy et al., 2016; Parker \& Hill, 2017). NPs are RNs with an advanced nursing education and clinical training who are licensed to diagnose and prescribe in the United States (Coombs, 2015). Because of their advanced nursing role, NPs can provide a wide range of patient care services.

The demand for NPs grew significantly in the recent years. Access to care was greatly improved with the adoption of the NP role (Kooienga \& Carryer, 2015). The NP role was especially transformative in the area of unmet primary care needs (Kooienga \& Carryer, 2015) as well as in meeting the needs of underserved populations (Siomos, Bavis, Swartwout, Danko, \& Delaney, 2016). NPs improved access to care through bringing health promotion and disease prevention to indigent and remote populations. Change in the awareness of NPs' contribution to a health care system (AACN, 2015), increase in complexity of patient care, and a shortage of primary care services further exaggerated the need for more NPs (Giddens et al., 2014). Because of the growing need for more NPs, the number of practicing NPs increased by more than 100,000 
since 2009 with the current total number of practicing NPs exceeding 234,000 (AANP, 2017a). Thus, the NP working force is growing quickly to satisfy the population need for health care access.

\section{Education and Licensure}

To accommodate the increased need for NPs, the number of NP programs also grew. There are currently about 350 NP programs in the United States (AANP, 2017b). One example is family nurse practitioner (FNP) programs, which are available in the United States. These programs include onsite, hybrid, and online options with online programs becoming especially popular in the recent years (AANP, 2016b). To be accepted to an NP program, the candidate is expected to complete a bachelor of science in nursing (BSN) degree and have some RN experience (Hevesy et al., 2016). The minimum requirement for NP licensure is to obtain masters in nursing from a state-approved program with a specialization that depends on the candidate's preferences (Hevesy et al., 2016). NP programs have strict admission requirements

for their potential candidates. When admitted, NP programs require NP student to meet eight NP competencies and complete at least 500 clinical hours (AACN, 2015; Hevesy et al., 2016). No additional requirements exist for NPs' training after graduation. While other professionals such as MDs have extensive residencies that last for years to close the gap between the school and practice, most NPs start practicing right after graduation (Hevesy et al., 2016). A national certification exam must be completed successfully after the NP completes the program of study. Though the number of NP programs has increased, the programs' structure of clinical experiences has remained without major changes since 1960's. To accommodate current needs 
for quality patient care, in addition to the growing number of programs, major structural changes may be needed for programs' adaptation. This is especially true given the evolution of the Doctorate of Nursing Practice (DNP) role. Gaining a deeper understanding of how current programs fit into the NPs' current role is necessary.

As NP programs have proliferated, various obstacles affected how many candidates can become NPs and where they can practice. While NP schools have been trying to accommodate the growing number of qualified applicants, their enrollment numbers have been restricted by the lack of clinical training sites (Drayton-Brooks et al., 2017; Giddens et al., 2014; Van Leuven, 2014). Without a sufficient number of clinical sites, programs are unable to provide students with quality education (Heitz et al., 2004). Further complicating the situation, NP licensure requirements lack consistency among the states. The NP role has been defined by such attributes as titles, the scope of practice, and legal role requirements (Andregard \& Jangland, 2014) and licensure criteria are state specific and vary among states (Hevesy et al., 2016; Scanlon, Smolowitz, Honig, \& Barnes, 2015). All 50 states have different NP licensure criteria, creating a barrier for NPs practicing or training across the United States.

Uncertainty also exists regarding NPs' degree of autonomy. In addition to the lack of training sites and inconsistency in licensure requirements among the states, disparities exist among the states regarding the prescription authority of advanced nurses and how much supervision NPs must obtain when prescribing (Parker \& Hill, 2017). Further, the amount of supervision NPs may require has been a topic of a long debate regarding NPs' full practice authority. This debate relates to whether NPs should practice autonomously without MDs' 
supervision or not. Opinions vary among the states and lack of standardization of the advanced nursing role among the states with all 50 states having own regulations, is due to the differences in the role understanding among the states (Kooienga \& Carryer, 2015). This variance in requirements for NP role is the reflection of uncertainty about the NP role and the level of NPs' readiness for practice after graduation.

\section{Collaborative Agreement with Physicians}

Besides affecting NPs' ability to practice across the states, lack of agreement on the role definition and NP scope of practice also affected NPs' collaboration with other professionals, which has negatively influenced knowledge about NP role. More specifically, this lack of consensus and standardization on the NP role caused confusion among other health care providers and became a barrier to international collaboration, data collection and generating knowledge about the role between the countries (Kooienga \& Carryer, 2015). Lack of clarity in NP role definition may also be responsible for friction between the NPs and other disciplines (Andregard \& Jangland, 2014). Though there is confusion about the NP role, safety and effectiveness of NPs' patient care have been well researched and documented (Griffin \& McDevitt, 2016; Harkey, Little, \& Lazear, 2017). Confusion about the NP role, however, may translate into the issues with collaboration and role transition after NPs graduate.

NPs' collaborative agreements with MDs may be the only source of preceptorship for many novice NPs. In most states, NPs do not have full autonomy as they have restricted or limited practice authority (AANP, 2017c). In 28 states, NPs must have a collaborative agreement with the physician prior to practicing. The collaborative agreement defines MDs and NPs' 
relationship and their legal requirements (Herman \& Ziel, 1999). Thus, an NP should be able to consult the collaborative MD as needed. Physicians are expected to lead and mentor NPs in a work environment (AANP, 2017c; American Medical Association, n.d.; Hallas, Butz, \& Gitterman, 2004). While in many cases, restrictive laws limit the NP's ability to provide patients with comprehensive care (Harkey et al., 2017); the rationale for having collaborative agreements is for MDs to provide NPs with guidance, which should help to close the gap between the school and practice. Further, the rationale provided for such restrictions includes the need for physician supervision due to NPs' limited education when compared with the physicians' education (Harkey et al., 2017). NPs graduate education lasts from two to three years depending on the program; compared to nine years that physicians spend to complete specialty education, medical school, and residency (Coombs, 2015). Though most states require collaborative agreements, no evidence is available to confirm that physician oversight improved NP patients' outcomes (Hansen-Turton, as cited in AACN, 2015; Safriet, as cited in AACN, 2015; Newhouse, 2011 as cited in AACN, 2015). While the MD-NP collaborative agreement restricts NP's scope of practice to ensure safe and effective patient care, the evidence is lacking to support the effectiveness of such collaboration. Without having any evidence supporting the effectiveness of such collaboration, it is difficult to say if such collaboration is helpful in NPs' transition to practice.

\section{Nurse Practitioner Role Transition}

The NP role transition to practice has been recognized as difficult, uncomfortable, and stressful. An NP's transition to practice is characterized by the high level of vulnerability and 
distress (Barnes, 2015a; Brown \& Olshansky, 1997; Flinter, \& Hart, 2016; MacLellan et al., 2015; Steiner et al., 2008). The difficult transition to practice may be related to the gap between the NP education and what is expected in practice (Fitzpatrick \& Gripshover, 2016). Gaining an in-depth understanding of NPs' role transition may assist in highlighting the areas that can be addressed to improve NPs' transition to practice process. However, limited research is available on the role transition of NPs (Faraz, 2016). NPs' role transition process can be affected by numerous factors that occur over time. Thus, it becomes important to explore transition stages.

\section{Transition stages}

NP role transition is a complex process. Poronsky (2013) pointed toward the significance of exploring the stages when studying NP role transition. An NP's transition may not be "stepwise or linear;" rather it is more multilevel and complex (Poronsky, 2013, p. 353); because it is complex, it is important to examine each factor's influence on each transition stage to understand the process in depth. Heitz et al. (2004) identified two stages of the RN to NP transition. The first stage begins with the acceptance to the NP program and lasts until the graduation; the second stage is marked by NP's graduation, and it lasts up to two years postgraduation (Heitz et al., 2004). Additionally, Schlossberg (2011) identified three transition stages of "moving in, moving through," and "moving out," followed by the fourth "moving in again" stage (see Figure 1; Goodman et al., 2006, p. 166). The initial role transition stage, from the time NP program starts to the graduation point, explained by Heitz et al. (2004) is further subdivided in Schlossberg's transition theory into the "moving in, moving through," and "moving out" role transition stages (Goodman et al., 2006, p. 166). The moving in again transition stage in 
Schlossberg's transition theory is equivalent to the Heitz et al. (2004)'s second post-graduation stage. The NP's ability to transition to his or her new role may change depending on the transition stages.

While role transition is one cohesive process, it is important to understand the details of how it occurs over time. During the moving in stage, one learns a new role, new routine, and assumes new responsibilities (Goodman et al., 2006). The moving in stage includes orientation, learning new skills, and feeling "marginal" (Goodman et al., 2006). During this stage, NP students learn the responsibilities of an NP student. Next, the moving through stage is characterized by a major change that translates into the new norm, which in turn, culminates into satisfaction with the new role (Goodman et al., 2006). During this stage, people may experience the period of being in between the two roles (Goodman et al., 2006). This moving through transition stage is the stage of an intense learning process of the new role while attempting to transfer some of the old role's skills (Goodman et al., 2006). During this time, NP students realize that while some RN skills may be transferable to their new role, many are not. The moving out stage is accompanied by the sense of loss and grieving process even when the change of roles is voluntary (Goodman et al., 2006). During the moving out stage, NPs graduate from their program, separate from their $\mathrm{RN}$ role, and start a job search. The post-graduation moving in again stage follows the moving out stage; it includes analysis of employment choices and making decisions regarding the job (Goodman et al., 2006). During the moving in again stage, the NP enters the first NP job and becomes a novice NP. During this time, orientation and frequent feedback are imperative for NPs' learning a new role (Cusson, \& Strange 2008). Exploring NP 
role transition stages is a complex process; however, it may be useful in identifying various role transition resources. Clearly, NP transition is a multistage process that requires support (MacLellan et al., 2015). Studying all transition stages is recommended since NPs perceive their transition as one process (Steiner et al., 2008). In this study, I explored the NPs' role transition related to the preceptorship during NPs' transition stages. Exploring NPs' experiences with transition and preceptorship during the transition stages is important for an in-depth understanding of a transition process.

While many researchers focused on NPs' role transition stage, which occurs after graduation, understanding the NPs' experience with the role transition in academia during the initial stage is also important for NPs' successful transition to practice. If an NP student failed to begin role transition during their academic program, his or her transition after graduation might be more difficult (Poronsky, 2013). Thus, it is important to study the entire transition period, before and after graduation to understand those factors that influence this complex process.

\section{Transition, Preceptorship, and Resources}

The NP role transition is a complex process. Operationalizing the abstract concept of an NP transition assists in gaining an in-depth understanding of what role transition entails for NPs. An NP's transition to a new role involves intensive learning through learning of a new role, assuming of an increased level of autonomy and responsibility, attempting to reconcile the two identities of a nurse and a provider, as well as overcoming many different emotions (Barnes, 2015b). Understanding each NP role transition attribute is necessary to learn about this process. 


\section{Transition Attribute: Role Learning}

Learning the new role involves gaining knowledge from a preceptor through experience, both before and after graduation (Barnes, 2015b). To learn effectively, NPs must know the content or have the necessary baseline knowledge, the ability, and the desire to learn (Collins et al., 1991). During the school years, NP students are involved in didactic courses to ensure that they have learned needed content. Additionally, students learn from their clinical preceptors and clinical experiences. After graduation, learning continues through collaboration, preceptorship, and self-learning by using the available resources. Role learning also occurs as a result of a preceptor using the following teaching strategies, (a) role modeling (trainees observe their preceptor's performance), (b) coaching and scaffolding (preceptor observes and assists trainees in performing), (c) articulation (trainees articulate their knowledge explaining their problemsolving strategies), (d) reflection (having trainees reflect on their performance as well as preceptors providing feedback), and (e) exploration [gradually removing supports to allow trainees for more independence] (Collins et al., 1991). Thus, the trainee NP observes the preceptor performing a task, and then the trainee performs a task under the preceptor's supervision and provides the rationale for the task completed. Lastly, the NP trainee receives the preceptor's reflection and feedback on the trainee's performance and integrates that information with their own reflections on their experience. The final goal is for the NP trainee to perform independently.

A preceptor's role modeling must take place for the role acquisition to occur. To model the professional role, a preceptor must be familiar with the role. Role acquisition may be affected 
by the preceptor's inability to role model. Role modeling during the transition process is essential (Maten-Speksnijder, Pool, Grypdonck, Meurs, \& Staa, 2015). The role learning process may be affected by many factors, including that if the preceptor's profession is different from that of a mentee. Because of the significant differences between the two professions (MDs and NPs) and the lack of understanding of NP role by MD preceptors, how quickly the NP trainee adjusts to the NP role may be negatively affected.

In addition to the role modeling, coaching the trainee, while providing him or her with the support and feedback, allowing for articulation of the problem-solving strategies along with gradual independence are all essential functions of a preceptor (Collins et al., 1991). Thus, NPs' expectations of their preceptors' involvement and feedback can also affect how much feedback trainees receive. The amount of feedback an NP trainee receives is essential for growth in the new role. Those with high desire for feedback are more attuned their preceptors' cues (Ashforth \& Saks as cited in Barnes, 2015b). Active learning of new skills is important for a successful transition. To request feedback, NPs must have an awareness of their need for feedback in their learning. Exploring NPs' experiences with their preceptors' feedback during NPs role transition is necessary to understand the NPs' role transition process.

In addition to the content knowledge and teaching methods, context and learning environment are also important for the role acquisition during the role transition process. Thus, role learning also include sequencing and sociology (Collins et al., 1991). Role sequencing refers to increasing the complexity and diversity of trainee's learning experiences (Collins et al., 1991). NPs advance from history collection and assessment to diagnosing and prescribing. The diversity 
of various diagnoses also increases with the increase of trainee's comfort level. Sociology refers to the significance of the trainee's learning environment (Collins et al., 1991). The learning environment is determined by choice of a clinic and preceptor. Furthermore, physical space, communication, and the organization's values regarding NP students and novice NPs' education are all important in NPs' learning of a new role (Flott \& Linden, 2016). Additionally, NPs' familiarity with the site or a clinic is significant for successful learning of a new role (Flott \& Linden, 2016); which can be achieved through the orientation. Providing NPs with variety relevant clinical experiences as well as orienting NPs to their learning environment plays a key role in NPs' learning of a new role. While role learning may vary depending on NPs' experiences and environment attributes, it may also differ depending on whether NP's environment is pre or post-graduation.

Novice NPs' experience with role learning during their transition after graduation changes as their responsibilities change from those of a student to becoming a licensed provider. Because most NPs lack formal preceptors after graduation (Hart \& Bowen, 2016) and most NPs must have a collaborative agreement with MD to practice, the role of their collaborators becomes that of preceptors who then play an essential part in how novice NPs learn their new role. An MD collaborator's awareness of novice NP's scope of practice, sharing workload, finances, communication, and MD's previous experience with NPs will affect how NP and MD will work together (Schadewaldt et al., 2013), which may play a role in novice NPs' learning of the new role during the post-graduation transition stage. Therefore, exploring preceptorship during novice NPs' role learning may help understand the process of NP role transition. 


\section{Transition Attribute: Autonomy and New Responsibilities}

The realization of increased responsibilities and autonomy is especially evident during the second or moving in again transition stage after NPs graduate and start practicing independently. The moving in again stage is characterized by applying new knowledge and skills to a new role (Goodman et al., 2006). NPs' job satisfaction was closely connected to the NPs' ability to meet increased responsibilities and their sense of autonomy (Horner, 2017). Gradually preparing NPs to this shift in responsibility to prescribe care while they advance through role transition stages may influence how NPs transition to the new role.

Transition to a new role involves a significant change in NPs' duties. As NPs change from taking orders from physicians to taking over the care of a patient and giving orders, there is a shift to a greater autonomy and increased responsibilities in an NP's new role (Barnes, 2015b; Chang, Mu, \& Tsay, 2006). Becoming a "prescriber of care" is an important attribute of NPs' transition to practice process (Barnes, 2015b, p. 140; Cusson, \& Strange 2008). Taking on new responsibilities and increasing autonomy can only be done when NPs have strong clinical skills.

The realization of shifting one's responsibilities is an important hallmark of NP's transition process. The process of role transition involves shifting from the role of an experienced RN to the role of a novice NP; prescribing care elevates NPs' responsibility and autonomy of patient care (Barnes, 2015b). The time NPs start performing independently or semiindependently may mark the time when most NPs recognize the need to assume additional responsibilities (Cusson, \& Strange 2008). A preceptor's frequent feedback is essential at this time for a trainee to progress to a greater degree of independence (Marfell, Mc Mullen, Onieal, 
Scheibmeir, \& Hawkins-Walsh, 2017). The trainee reaches a higher level of independence when a preceptor exercises sequencing or increases trainee' role complexity and independence (Collins et al., 1991). Additionally, after graduation, novice NPs may advance to the higher sense of autonomy and increased responsibilities as they start sharing a workload with their collaborator physicians, become more aware of their scope of practice, and legal responsibilities (Schadewaldt et al., 2013). Preceptors can assist NPs in becoming more independent and autonomous.

During this time of shifting in NPs' autonomy and responsibilities, NPs realize that they now have a role of a prescriber. Prescribing medications is a learned skill. Limited evidence exists on the ability of NPs to prescribe correctly upon graduation; however, Sabatino et al., (2017) found that NP students made many errors when prescribing; thus, underlining the need for additional education. Novice NPs expressed having significant difficulties and lack of preparation in prescribing for elder adults with multiple comorbidities and multiple medications (Jones et al., 2015). Thus, novice NPs have a strong need in preceptorship to successfully transition to their new responsibilities.

While prescribing may be challenging, making a medical diagnosis is another complex and new skill for advanced nurses. Diagnostic errors occur with over five $\%$ or 12 million of the adult population being affected yearly (Singh, Meyer, \& Thomas, 2014). Additionally, more diagnostic errors are done by those who use the least time to complete patient visits (Pirret, 2016); NPs are often rushed to improve rate of return (ROR) time to make a profit and see more patients per day to justify their salary. Most of paid malpractice related claims against NPs were 
related to diagnostic and treatment errors (Sweeney, LeMahieu, \& Fryer, 2017). Thus, prescribing and diagnostic areas are potentially the two most important areas that may need additional support during the NPs' transition to practice. Teaching prescribing and diagnosing skills is an important function of preceptors. Sabatino et al. (2017) attempted to introduce a fourweek prescribing class to family nurse practitioner (FNP) students with improved results. Though students learned a lot about prescribing practices, their post-intervention errors were still above $30 \%$ (Sabatino et al., 2017).

While prescribing is a complex process of safely choosing the correct medication, the diagnostic process is also difficult. Diagnostic reasoning requires the use of intuition, pattern identification, and the use of the analytical approach. It was found that NPs are more likely to use intuitive diagnostic approach when compared with MD residents (Pirret, 2016). NPs' cognitive overload and fatigue that may be due to a difficult transition to a new role, especially when combined with the complexity of patient care and limited appointment time, may lead to diagnostic errors (Pirret, 2016). Additionally, clinical expertise plays a significant role in avoiding diagnostic errors (Sherbino \& Norman, 2014). Exploring NPs' experiences with how NPs' preceptors assisted them with developing their diagnostic reasoning skills during their role transition was essential in understanding NPs' transition to practice process. While changing responsibilities is characteristic of NPs' role transition, professional identity confusion is also common during NPs' role transition. 


\section{Transition Attribute: Reconciling Two Identities}

Forming a new professional identity, reconciling the roles of a nurse and a provider is an important step in NPs' transition to a new role. "Straddling two identities" is one of the transition process defining attributes (Barnes, 2015b, p. 139). NP students are admitted to the graduate programs as highly skilled and autonomous RNs. Role transition starts with RN learning about his or her new advanced nursing role. Loss of professional identity and regression of competence and confidence was noted in the first several months of the graduate program (Roberts, Tabloski, \& Bova, 1997). The realization that many RN skills may not be transferrable to the NP role may cause NP students to lose confidence. Also, being "in limbo" in between the role of an RN and the role of a provider while experiencing the feeling of being an imposter as well as "feelings of chaos, turmoil, and confusion" is characteristic of NP transition process (Brown \& Oshlansky, 1997; MacLellan et al., 2015, p. 394). Perception of being an imposter while struggling to reconcile the role of a nurse who provides care and a provider who prescribes care can lead to self-doubt and high levels of stress in a novice NP (Brown \& Olshansky, 1997). Additionally, being positioned between the two roles may cause identity confusion (Jangland, Yngman Uhlin, \& Arakelian 2016). Both before and after graduation, the NPs' sense of role identity may be affected by his or her experience with their preceptorship. To successfully transition to a new role, NPs may need guidance with developing a new professional identity; new role's responsibilities require NPs and their preceptors implement supportive strategies. Examining NPs' experiences with how their preceptors facilitated the usability of NPs' previous skills and 
knowledge during their transition to practice was helpful in the understanding of NPs' transition process.

While struggling to find their new role identity, NPs are often guided by MDs. Often physicians are assigned to be NPs' collaborators. For those novice NPs who were provided with preceptorship, most preceptors were represented by MDs though MDs have a different educational background (Hart \& Bowen, 2016). However, one of the essential functions of a preceptor is role modeling (Collins et al., 1991). Attempts of modeling the role after physicians were unsuccessful (Roberts et al., 1997). Thus, exploring NPs' transition experiences while being trained by different preceptors helped illuminate important attributes of their experiences.

Furthermore, confusion between the medical skills that must be learned to perform an advanced nursing role may further cause NP students and novice NPs struggle with their new role identity. The NPs' role appears to be blended into medicine and nursing creating confusion and friction on both sides of this professional spectrum (Andregard \& Jangland, 2014).

Resistance and hostility from RNs and physicians must be overcome by NPs, as the NP role includes the characteristics of both professions (Fitzpatrick \& Gripshover, 2016; Jangland et al., 2016). NPs may tend to regress into the $\mathrm{RN}$ role during their clinical experiences and assume $\mathrm{RN}$ responsibilities (Roberts et al., 1997), “stepping back in expertise” (Brown \& Olshansky, 1998, p. 55). Professional identity is important in one's role transition. However, education on how to integrate the two roles, medical and nursing, is currently not provided in NP schools (Jones et al., 2015). Thus, NPs' awareness of the role reconciling concept along with strategies of how to successfully adapt the new role is necessary. NP students and novice NPs experience feelings of 
incompetence, lack of prioritizing skills, as well as confusion between the medical and nursing models (Roberts et al., 1997). Reconciling the two roles and new role identity development is necessary for successful role transition. In the absence of formal education on how to reconcile the two roles, it was important to explore the NPs' role transition experiences with preceptors related to the development of their new professional identity.

While attempting to reconcile nursing and medical models of patient care, NPs are also faced with having to care for sicker patients in a shorter period of time. Being faced with complex patients who present with multiple complaints and numerous chronic health issues creates more self-doubt (Graue et al., 2015); multiple patient problems' prioritizing appears to be an issue for NP students (Roberts et al., 1997). Doubting one's own skills and abilities leads to further identity confusion. Because of the feelings of incompetence at the start of students' clinical experiences, NP students often express a desire to observe their preceptors for several weeks instead of performing NP students' functions (Roberts et al., 1997), which further decreases the number of clinical hours for students to learn their new skills. Learning new assessment skills, shifting the focus to medical diagnoses, and needing to see large numbers of patients cause NP students to shift to a less known medical model, which exaggerates their role confusion (Roberts et al., 1997). Preceptors are in the unique position to assist NPs in gaining confidence while they are transitioning to a new role.

NPs' identity confusion is further exacerbated by the difficulties NPs experience separating from an expert RN role. However, during NP role transition, to identify with the new role, it is imperative for an NP student or a novice NP to separate from an old RN role to focus 
on the transition to a new role (Barnes, 2015b). Heintz (2004) reported that NP students' role confusion and role separation factors had an effect on NPs' role transition. Additionally, NP students who performed roles other than the one of an NP student, such as one of an employee, reported more difficult role transition (Heitz et al., 2004). To gain the new role identity, NPs may need guidance on how to separate from an $\mathrm{RN}$ role. Through coaching and reflection strategies, preceptors have an opportunity to help trainees to develop the skills needed for them to function in a new role (Collins et al., 1991). More specifically, NPs' preceptors can guide NPs regarding which RN skills may be transferable to their new role and which may not. However, with most NPs' preceptors being MDs who are less familiar with NPs preparation level and educational requirements (Fitzpatrick \& Gripshover, 2016; O'Brien, Martin, Heyworth, \& Meyer, 2009), the process of role separation may be delayed. Further, MDs expect NPs to function at an expert level right after graduation (Fitzpatrick \& Gripshover, 2016; O'Brien et al., 2009). This lack of familiarity with the NP role and unrealistic expectations may impede the NPs' desire to separate from their RN role delaying the development of a new role identity. Focusing on a new NP role can provide an NP with more time and satisfaction with the new role; however, it may not be feasible for those NP students or new graduates who must continue working in an RN role needing a stable source of income (Parker \& Hill, 2017). Learning about NPs' experiences with the role their preceptors have in helping them to effectively disengage from their previous $\mathrm{RN}$ role was necessary to understand NPs' role transition. 


\section{Transition Attribute: Conflicting Feelings}

In addition to learning a new role, shifting from a nurse to a provider, and struggling to reconcile between the two identities, NPs experience many mixed feelings during their role transition. Among the eleven emotions identified in transitioning of NPs, ten were reported as negative (Barnes, 2015b). These emotions often included feelings of "fear, insecurity, being overwhelmed, inadequate, vulnerable, and isolated" (Heitz et al., 2004, p.417). Novice NPs' feelings of being an imposter (Brown \& Olshansky, 1997) discovered in earlier research have been confirmed by identifying the role ambiguity and identity confusion feelings in new NP graduates (Barnes, 2015b; Faraz, 2016). Preceptors have an opportunity to coach trainees to help them gain the confidence necessary for performing in a new role (Collins et al., 1991). Feeling confident in own skills is important for successful role transition. It was necessary to research NPs' emotional experiences during their role transition when in a preceptship.

Feelings of not being able to handle the new responsibilities of an NP may jeopardize the NPs' role transition and cause some NPs to leave affecting the retention of NP workforce. NPs' feelings of self-doubt and incompetence as well as mixed negative feelings were caused by their expanded responsibilities (Hart \& Macnee, 2007; Heitz et al., 2004). Struggling with the new responsibilities, trainees begin to search for the ways to cope doubting self; this creates fears of never being able to function in a new role (Roberts et al., 1997). Loss of confidence may affect NPs' retention. More than a quarter of novice NPs will leave their job within one year (American Association of Colleges of Nursing, [AACN], 2015). Both preceptors and organizations may be able to offer NPs the needed support. Organizational support was essential to NPs' successful 
transition to practice (Dillon, 2016). Trainees' learning environment is important for their mastery of new skills (Collins et al., 1991). More specifically, both prior and after graduation, the amount of support provided by the leadership as well as sharing the workload can be facilitated by NPs' preceptors and the organization. Transition support is essential in helping NP students, and novice NPs feel comfortable and competent in their new role. As NPs' emotions constitute an important part of their role transition process, exploring the NPs' experiences with how their preceptors nurture them during their role transition was needed to provide NPs, schools, and employers with appropriate tools to support NPs' transition to practice process.

\section{Transition Resources}

The support available to NPs is important to provide the necessary structure for preceptors to assist NPs with their role transition. Schlossberg's (2011) transition theory was helpful in guiding this portion of the literature review. More specifically, NPs' support systems may be divided into the four "S's," situation, self, support, and strategies (Goodman et al., 2006). NPs' transition experience also depends on the degree of personal and organizational support available (MacLellan et al., 2015). Support systems such as well-structured orientation and preceptorship can improve NPs' self-confidence, skill acquisition, job satisfaction, and socialization into the new role (Barnes, 2015a). Providing a support structure that is based on available resources for NPs both prior and after graduation can have a profound effect on their role transition.

Situation. Because NPs' transition can be altered by the environment, it becomes necessary to examine the situations that surround NPs' role transition. NPs' role transition is 
triggered by NPs enrolling into the graduate nursing program and then seeking a new job. These events lead one to change his or her situation (Goodman et al., 2006). One's transition and the ability to learn may change depending on the individual's ability to afford the cost of the education, and the time one has available for learning. Heitz et al. (2004) identified personal commitments and additional roles as extrinsic barriers to NPs' role transition during their first transition stage. Most NP students continue working in the role of an RN, which may limit their time available to study. Similarly, after graduation, time dedicated for novice NPs' learning may be limited due to high demands to fit more complex patients in one day. Having to care for complex clients may cause novice NPs to doubt their skills (Graue et al., 2015). What is expected of the new graduates in the work environment changes from that of academia (Lindfors, Hultell, Rudman, \& Gustavsson, 2014). NPs employers' expectations of having a “profitable” NP may also decrease NPs' focus on their learning of a new role. Demands for high productivity may cause novice NPs to quickly self-educate on doing unfamiliar things or procedures. In fact, $47.7 \%$ of NPs felt that they were practicing outside their scope of practice during their first year of practice (Hart \& Bowen, 2016). Thus, time and costs may influence NPs' learning opportunities. More specifically, learning the content necessary to function in a new role along with receiving and using feedback from the preceptors, may be affected by the time and cost factors. Learning how preceptors supported NPs in their time management to help their transition may be necessary.

Additionally, role transition may be affected by how much control one has over the transition circumstances (Goodman et al., 2006). Those who are more adaptable have developed 
networks, and healthy may have better control over their transition (Goodman et al., 2006), making the learning of a new role an easier process. Heitz et al. (2004) identified NPs' personal support systems including family, friends, and coworkers as extrinsic facilitators of role transition during the initial stage prior the graduation. Furthermore, Heitz et al. (2004) pointed out the support by the colleagues and collaborative MDs as extrinsic transition facilitators during the role transition after graduation. Thus, depending on NP's situation, he or she may have different support systems in place to facilitate the role transition. Learning preceptors' support in accommodating NPs' personal situations assisted in understanding the NPs' role transition process.

Further, previous situations and experiences with the learning of a new role may be helpful in one's current transition experience (Goodman, et al., 2006; Heintz et al., 2004). While Steiner et al. (2008) found that prior life experiences may facilitate NPs' role transition. Barnes (2015) was not able to detect the significant relationship between previous RN experience and NPs' role transition. Additionally, in earlier research, Roberts et al. (1997) noted prior experience that included other graduate experiences with the relativism, limitations of existing resources, and the ability to treat gray areas within the informational sources may contribute to an easier transition experience. Overall, it is important to consider situational factors such as why one is learning a new role, the time and money one has available for learning, one's ability to adapt to a new role, as well as one's previous experience with similar transitions and their relationship with NPs' preceptorship. 
Self. Person's unique characteristics may also influence his or her role transition. First, the significance of the new role in one's life or work life balance may be important in how one experiences learning of the new role (Goodman et al., 2006). Second, one's resilience may determine how one can adapt to a new role (Goodman et al., 2006). Third, self-efficacy or perception of abilities may also influence one's learning of a new role (Goodman et al., 2006). Lastly, a match between values and work may also affect the learning of a new role (Goodman et al., 2006). Heitz et al. (2004) found that optimistic self-talk is an intrinsic role transition facilitator during all transition stages. The researchers also found that negative feelings related to self-perception and role confusion, as well as being in between the two roles, were intrinsic barriers of NP students' role transition; however, role separation during the first transition stage and role acceptance during the second stage were found to be intrinsic role transition facilitators (Heitz et al., 2004). Overall, the attributes of a new professional role related to an individual's characteristics including work-life balance, resilience, adaptation, and meaning can all influence NPs' role transition and may be supported by preceptors.

Support. To be successful, NPs' role transition must be well supported prior and after graduation. Support is often provided by people who encourage, teach, provide contacts, or assist with immediate needs such as transportation, finances, or babysitting (Goodman et al., 2006). Heitz et al. (2004) identified initial stage extrinsic role transition facilitators related to NP students, which included a preceptor's guidance and role modeling, faculty, family members, friends, and organizations. At the same time, identified extrinsic role transition barriers included factors related to the events at the clinical training sites as well as preceptors' teaching styles 
(Heitz et al., 2004). Effective preceptorship is important for a trainee to learn his or her role successfully (Marfell et al., 2017). Exploring NPs' experiences with the role of NP students' clinical training sites and their preceptors' teaching styles in supporting NP students' role transition was imperative in understanding the role transition process during the initial transition stage that occurs prior the graduation.

Supporting novice NPs' role transition that occurs after graduation is just as important. Heitz et al. (2004)' study was pivotal in describing extrinsic facilitators and barriers of novice NPs' role transition after graduation. More specifically, Heitz et al. (2004) described collegial and physicians' support, networking, and approval by lay people as important role transition facilitators. At the same time, Heitz et al. (2004) identified negativity from the colleagues, finding a job, and having to constantly educate others on NP role as extrinsic barriers to successful role transition after graduation. Therefore, novice NPs' colleagues and collaborators may have a significant effect on how NPs transition to their new role.

While some organizations attempt to provide support and offer formal orientations to novice NPs, it is important to understand that preceptor-trainee relationship differs from the formal orientation (Marfell et al., 2017). While orientation may be provided in the form of a course to support a trainee, preceptorship is a one-on-one relationship between the experienced professional and the beginner (Marfell et al., 2017). Such a relationship, when effective, may significantly facilitate one's learning the new role; thus, assisting in effective role transition (Marfell et al., 2017). Preceptors must be chosen with care to support novice NPs' role transition. Orientation programs should not be used in place of preceptorship. It was essential to investigate 
the degree and type of preceptorship support novice NPs experienced during their role transition after graduation.

Strategies. How NPs learn their new role may also vary depending on what coping strategies they use to adjust to a new role. Adults in transition use various coping strategies to change, adjust, or to help with stress during the adjustment period (Goodman et al., 2006). A good match between the trainee and the preceptor is important for successful role learning (Marfell et al., 2017). More specifically, interests, schedules, and interaction styles need to be considered when choosing a preceptor (Marfell et al., 2017). A good match between a student, clinical site, and a preceptor may facilitate NP students' role transition (Heitz et al., 2004). After graduation role immersion, networking, and approval by others may facilitate novice NPs' role transition (Heitz et al., 2004). Steiner et al. (2008) followed Heitz et al.'s (2004) study and showed that "agreement was found with prior life experiences, preceptor guidance, personal support system, optimistic self-talk, role modeling, and hands-on laboratory experiences” (p. 445). The effectiveness of NPs' strategies may also depend on the quality of preceptor's advice. Investigating strategies NPs use to help to learn their new role from a preceptor was essential in understanding NPs' role transition process.

Pre-graduation resources. Though little is known about NPs' experiences with the transition while in a preceptorship, the significance of preceptorship in NPs' transition has been noted in some studies. Heitz et al. (2004) found faculty guidance to be the primary positive force in NP students' role transition; thus, the influence of faculty on NP students' transition process in an academic environment should also be acknowledged. However, in Steiner et al.'s (2008) 
study, clinical preceptor guidance was found more significant. Preceptorship appears to be one of the most significant, though least supported, elements in NP students' transition. Students' fear of not knowing enough because of not having exposure to sufficient number of clinical situations during their clinical rotations led to students' feelings that their preceptor and sites were inadequate for their learning needs (Roberts et al., 1997). Exploring the elements of preceptorship in relation to NP students' role transition was needed if changes in clinical education are to be made.

Clinical education. To better understand the resources available to NPs prior to graduation, the current state of clinical education was examined. While the number of NP schools has increased dramatically in the recent years (AACN, 2015), current clinical education is outdated and does not meet the requirements of current health care environments. The one-onone preceptorship model used for NPs' clinical education is the same as the one used 45 years ago when a much lesser number of NPs graduated yearly (AACN, 2015; Giddens et al., 2014; LeFlore, \& Thomas, 2016). More specifically, current concerns regarding NP students' clinical preparation include (a) the degree of students' preparation prior starting their clinical experiences, (b) lack of preceptors' compensation, lack of preceptor training, and lack of employers' accommodations for preceptors to precept, (c) lack of clinical sites, faculty, and preceptors, (d) inadequate knowledge of NP educational program and scope of practice by MD preceptors, (e) lack of interdisciplinary clinical education elements, and (f) lack of tools and consistency in objective evaluation of students' clinical performance. Understanding how to 
strengthen current NP clinical education required exploring NPs' transition to clinical practice in relation to preceptorship.

Supporting NP students by providing them with quality clinical education during their initial role transition stage is necessary to ensure NPs' smooth transition to practice adjusting to a complex and demanding PC clinical environment. During NPs' role transition in academia, curricular issues of inadequate preceptorship and lack of clinical time were identified (Heitz et al., 2004; Steiner et al., 2008). However, quality clinical education is necessary for NP students to feel competent and prepared to practice, especially in providing care to complex primary care clients with multiple comorbidities and limited resources. The need for NPs grew due to the increased physician shortage, recognition of NPs abilities as advanced nursing providers, and reduced medical residents' hours (Keough et al., 2015; Roberts et al., 2017). The need for adult PC providers was predicted to grow by $29 \%$ from 2005 to 2025 leading to the projected shortage of PCPs up to 40,000 providers (Sroczynski \& Dunphy, 2012). Clearly, the need for primary care NPs is growing. However, the number of specialty NPs is increasing with current estimates being approximately one-third of all practicing NPs (Coombs, 2015). NP students' experiences with preceptors and clinical programs were especially important in students' comfort level and choice of PC as a future job (Budd, Wolf, \& Haas, 2015). Quality of clinical education may be responsible for NPs' employment choices. Providing primary care services is a complex skill, and many novice NPs may be intimidated by its complexity. New approaches to NP clinical education are needed to meet primary care need for increased number of NPs (LeFlore, \& 
Thomas, 2016). Supporting NPs' role transition through strengthening NP students' clinical education is necessary.

One of the important contributing factors to the issue of quality of NP clinical education is the lack of consistent competency based evaluation strategies to assess NP students' readiness for practice. The number of clinical hours is essential to students' learning; however, the quality of students' clinical experiences may also affect their role learning. While at least 500 clinical hours is required for each student by the graduate nursing programs (AACN, 2015; Hevesy et al., 2016), little is known about the effectiveness of students' experiences. In addition, no evidence exists that 500 clinical hours translate into required NPs' competencies (Hallas et al., 2012). Evaluating NPs' competence based on the number of clinical hours may be insufficient (LeFlore, \& Thomas, 2016). Changing the focus from an hour-based to a competency-based clinical evaluation may be necessary (Schumacher \& Risco, 2017; Sroczynski \& Dunphy, 2012). Furthermore, evidence showed that $38 \%$ of NP program representatives expressed concerns with an inadequate number of medical conditions students are exposed to through their clinical education (Erikson et al., 2014). Ensuring competence at regular periods of time during NP students' experience is essential for them to learn the information necessary to function in their new role with confidence. Quality of clinical experiences depends on many factors. These factors include clinical environment, students' preparedness, and preceptors.

Clinical learning environment. Clinical learning environment (CLE) is usually located outside of school and includes hospitals and clinics. CLEs give students an opportunity for hands-on experiences (Erikson et al., 2014). CLE includes the following characteristics. First, it 
is defined by a physical space that must contain the necessary equipment for a student to practice new skills (Flott \& Linden, 2016). The second characteristic includes student's communication with the preceptor and CLE staff, which can have a profound effect on student's learning outcomes (Babcock, Rosebrock, \& Snow, 2014; Flott \& Linden, 2016). Third, CLE organizational culture and policies effect student's learning experience (Flott \& Linden, 2016). For example, policies that limit student experience to observation only or organizational culture and leadership style that encourages quality care and students' learning in the CLE (Flott \& Linden, 2016). Lastly, preceptor's instructing strategies, student's engagement, as well as patient care opportunities, have an effect on student's learning (Flott\& Linden, 2016). Subsequently, to learn their new role, NP students need to have a CLE that is equipped to their needs; they need to have some familiarity with their preceptors and staff to ensure that the communication is clear and effective; students also need to be familiar with the practice's policies and the types of diagnosis and patient population usual for the site. Furthermore, NP students must be familiar with the teaching style and the expectations of their preceptors, making sure that preceptors are also aware of the students' expectations. Lack of adequate clinical sites was found to be a significant role transition barrier (Heitz et al., 2004). Exploring NPs' experiences with their CLEs is central to understanding their role transition experience. While the role of CLE is vital in students' learning of a new role, many challenges exist in securing and maintaining such sites. Many nursing schools are having difficulty securing the clinical sites. Challenges of obtaining clinical sites and preceptors are well documented (Drayton-Brooks et al., 2017). However, CLEs or clinical teaching sites are important to students' learning as students spend a 
lot of hours there to learn their clinical skills (Flott \& Linden, 2016). To identify issues with clinical sites within the MD, DO, NP, and PA programs, the first survey of this kind was mailed to the deans of related programs (Erikson et al., 2014). The MD, DO, NP, and PA schools experienced concerns regarding the declining number of clinical sites (Erikson et al., 2014). Obtaining primary care sites has been a great issue for 34 to $60 \%$ of NP programs (Erikson et al., 2014). While the number of programs grew, declining number of clinical sites affected the enrollment numbers (Erikson et al., 2014; Forsberg et al., 2015; Wiseman, 2013). Further affecting the enrollment numbers of qualified NPs was the shortage of doctoral prepared faculty (AACN, 2015). Examining the reasons for the challenges related to securing and maintaining clinical sites was necessary for creating adequate role transition support structures.

NP program leaders reported the following barriers to attaining new training clinical sites. Eighty-one percent of leaders expressed concerns regarding legal and security issues, which were central in securing sites (AACN, 2015; Erikson et al., 2014). More specifically, these issues included EMR access, background checks, drug screens, HIPPA training, and immunizations (AACN, 2015; Erikson et al., 2014). In addition, the complex process of establishing affiliation agreements between the academia and practices with an increased amount of documentation required was another barrier of securing clinical sites (AACN, 2015). The site agreements process delayed students' onboarding at the clinical sites (Roberts et al., 2017). Seventy-four percent of leaders expressed issues with training preceptors; $75 \%$ were concerned with students' issues of accessing EMR, and 64\% expressed that ensuring students are trained in EMR use was an issue that decreased the university's ability to obtain a new clinical site 
(AACN, 2015; Erikson et al., 2014). Because most sites utilize EMR system for the documentation purposes, students are commonly required to complete EMR training and receive login information, which can cost several thousands of dollars per each student (Forsberg et al., 2015). In addition to security responsibilities when establishing a clinical site, one must complete contract paperwork that may be a lengthy process requiring multiple approvals (Forsberg et al., 2015). Furthermore, the amount of documentation has increased, especially when students attempt to secure out of state clinical site (AACN, 2015). Overall, the process of securing and maintaining CLE sites has many challenges including time-consuming lengthy documentation and expensive security processes that are hard to overcome.

Unintended competition. Besides the difficulties with meeting numerous clinical sites’ requirements, unintended competition for clinical sites among the same and different professions is yet another obstacle to overcome when providing NP students with the quality clinical education. Along with the growth in the number of graduate medical education schools, the number of first-year enrollment also increased "to an overall 18\% increase in first-year enrollment at M.D.-granting medical schools, a 96\% increase in first-year enrollment at D.O.granting medical schools, a $215 \%$ increase in total enrollment at NP programs, and a $66 \%$ increase in first-year enrollment at P.A. programs from 2002 to 2012” (AACN, PAEA, \&AAMC as cited in Erikson et al.,2014, p.4). From 2010 to 2015, the NP enrollment numbers grew 81\% (AACN, 2015). Additionally, online schools and off-shore schools worsen the competition for clinical sites (Erikson et al., 2014). The competition for clinical sites increased between and 
within the professions (AACN, 2015; Giddens et al., 2014). With an increase in site competition, leaders struggled to come up with solutions on how to ensure NP students' quality education.

With the growing NP enrollment numbers, the demand for clinical sites and preceptors grew, challenging the program leaders to consider compensation for using clinical sites. However, only $4 \%$ of NP schools paid a fee for their students attending practicum (AACN, 2015). Though few schools compensate clinical sites and preceptors, the most common incentive important to preceptors was found to be financial compensation (Roberts et al., 2017). Not having sufficient number of clinical preceptors was the primary reason for not accepting qualified candidates into the nurse practitioner programs (Fang, Li, Arietti, \& Bednash, 2014). In fact, $96 \%$ of NP program representatives were concerned with the inadequate number of clinical sites; $94 \%$ and $84 \%$ expressed concerns about having enough qualified primary care and specialty preceptors respectively (Erikson et al., 2014). Sites are often bombarded by schools and students with numerous requests for preceptors (Forsberg et al., 2015). Students frequently feel frustrated when the responsibility to find a clinical site is placed on them (Forsberg et al., 2015). With such deficit in finding appropriate clinical sites, it was necessary to explore the quality of preceptorship as it relates to the role transition. NPs' experiences with preceptorship and finding clinical sites are important in understanding their role transition. Because financial compensation of preceptors and sites was infrequent, academic leaders had to come up with some other innovative solutions.

Innovative solutions. To offset the lack of clinical training, educators had to come up with some creative solutions to increase the quality of students' clinical experience. To compensate 
for the lack of clinical sites, graduate nursing programs' leaders considered maximizing simulation laboratories and expanding the geographic areas for site searches (Erikson et al., 2014). Thus, rural clinics have been used by many nursing schools. However, the barriers of using rural sites included students' expenses related to travel (Kaplan, Klein, Skillman, \& Andrilla, 2016). Driving long distances to get to their clinical sites takes additional time and resources from NP students many of whom already struggle combining school with work. Further, low and high fidelity simulation in the form of supplies, case studies, computer programs, and standardized patients was increasingly used to compensate for the lack of clinical sites and preceptors (AACN, 2015). While simulation experiences may be helpful in students' learning of clinical skills, they are not the same as clinical immersion in real clinical situations. Further, the number of clinical sites in the rural areas may be limited; thus, while it may be helpful to expand the geographical area for such site searches, it may be a temporary and partial solution to a growing problem.

To further help solve the issue with lack of clinical sites, the graduate schools of nursing leaders have implemented the academia practice partnerships (APP). Because clinical training must be a joint effort between the academic institutions and practice sites (AACN, 2015; Giddens et al., 2014), effective strategies in adapting NP clinical education to the increasing demands for PCP providers include creating an APP (Sroczynski, \& Dunphy, 2012). Creating partnerships between the academia and practice may be more helpful than collaborating with preceptors alone as changing the employment by the preceptor may affect the relationship the school has with the clinical site (Forsberg et al., 2015). Though the significance of APP is well 
recognized, few such partnerships exist currently (Roberts et al., 2017). While the creation of academia practice agreements, reaching out to rural clinics, and enhancing simulation learning is important, these attempts were inconsistent, and school specific. Therefore, exploring NPs' lived experiences with the role transition related to their experiences with the preceptors and clinical sites was necessary to identify new meanings of NP transition to practice concept.

NP student preparation. NP students' degree of preparation for their clinical experiences varies. Many preceptors expressed concerns regarding students' preparation level questioning their preparation for practice (Roberts et al., 2017). Preceptors expressed the need for students to have core clinical knowledge of documentation, assessment, pharmacology, and health interviewing (Keough et al., 2015). For the experiential learning to take place, the following attributes must be present. Pts with specific diagnoses related to student's learning needs; student must possess the foundational knowledge needed to perform the skills; and preceptor must be able to transfer knowledge and demonstrate the required skills (Flott \& Linden, 2016). If unprepared, students may have inferior experiential learning outcomes. Lack of NP students’ baseline knowledge may translate into the lack of skills in novice NPs. In fact, novice NPs expressed concerns about the lack of training to perform procedures, such as suturing, fracture care, X-Ray readings, etc. (Jones et al., 2015). Preceptors also expressed frustration about the variability of NP students' preparation level; preceptors carry the burden of having students who have not been checked for clinical knowledge in their didactic portion of the program (Giddens et al., 2014). However, in current fast pace practice environments, preceptors' time is limited leaving trainees having to fill their gap in knowledge; however, to transition to a new role 
successfully, trainees must be able to translate didactic knowledge acquired in the classroom to the clinical environment. As a result, students may be "stepping back in expertise," (Brown \& Olshansky, 1998, p. 55), and regressing to the role of an RN (Roberts et al., 1997). Therefore, students' preparation level is essential for the success of their preceptorship. It was vital to explore NPs' experiences with their preparation level prior their clinical experiences during their role transition.

To be appropriately prepared to function at the clinical site in a role of an NP student, it is also important to have an opportunity to orient to the new clinical environment. To assist students in preparation for their clinical experiences preceptors should provide students with the information related to the clinic's patient population and most frequent diagnoses (Babcock et al., 2014). Providing such information about the clinic is vital because most of the students are RNs whose background often includes inpatient nursing only. Thus, learning how an outpatient clinic operates may help students to acclimate to the new learning environment. In the absence of a formal orientation; however, students frequently must show the initiative to orient themselves to the clinic. Students' initiative to learn was noted as important by the NP preceptors (Keough et al., 2015). Whether initiated by the school or by a student, orientation to the site is a necessary step. Both students and preceptors' effort are central to students' preparation for their learning experiences. Though site orientation may largely depend on the chosen clinical site process and student's initiative, the role of NP faculty is also important.

Academic educators also have a key role in preparing NP students for their clinical experience by implementing competency-based instead of hour-based evaluation. Though faculty 
shortage is a current problem (AACN, 2015; Giddens et al., 2014; LeFlore, \& Thomas, 2016; Van Leuven, 2014), implementing standardized assessment of students' skills may lead to increased providers' confidence in students' skills (Giddens et al., 2014). However, the ability to evaluate students' progress has additional challenges when NP students are paired up with the MDs as their clinical preceptors (Andregard \& Jangland, 2014). The MDs' lack of knowledge of how NPs close the gap between school and practice may be related to the inconsistencies in how the provider's competencies are evaluated. MDs do not always know NPs' scope of practice. Bargagliotti and Davenport (2017) underlined the importance of using the standardized language that will be understood by all providers to assess NPs' competencies and improve NPs' evaluative practices. One example of such evaluation is using entrustable professional activities (EPA) or specific, measurable outcomes and milestones at the specific times of NPs' education (similar to check-offs used in undergraduate nursing education), which may provide such confidence to other providers in NPs' abilities to perform (Bargagliotti \& Davenport, 2017). EPAs would provide the foundation for a competency-based clinical evaluation of students, which is necessary to complete prior entering clinical site environment to ensure preparedness for practice in a new student role (AACN, 2015; Giddens et al., 2014; Keough et al., 2015). However, while broad-based competencies unique to each APRN role have been developed, currently, no nationally accepted assessment tools have been developed to support competencybased NP assessment (AACN, 2015). NPs lag behind other medical professions in abilities to assess students' preparedness for clinical experiences (AACN, 2015). To be eligible for the certification exam NP students must satisfy the course work and the number of required clinical 
hours (AACN, 2015) but no measurable competencies achievement is required. It is vital to not only ensure that students completed the required number of clinical hours but also to establish that learning of needed skills and procedures took place both prior to starting the clinical experiences, during, and upon their completion. The lack of measurable competency tools further complicates preceptors' assessment of their NP students' abilities and progress in learning of clinical skills.

To ensure the quality of students' clinical preparation, preceptor and site selection must be approached with care. Because students vary in their preparation level and the time they may require adjusting to their new role, special attention should be given to the process of matching the preceptor and the student (Forsberg et al., 2015). For example, sites with lower patient volume should be reserved for beginners, while fast-paced clinical environments should be selected for more advanced students. It may also be necessary to match preceptor and students' personalities and experiences. If the mismatch between the preceptor and the student occurs, both student and school can suffer (Forsberg et al., 2015). Close to 10\% of preceptors reported that the site selection was poor for the student's learning needs (Roberts et al., 2017). Because the selection is often made based on site and preceptor's availability along with student's home location, other important attributes of a good match are not taken into the consideration. For example, the majority preceptors expressed a desire to know more about their students' learning needs as well as conducting interviews with the students prior to making a commitment to train (Roberts et al., 2017). Along with other factors, students' learning needs must be considered 
when selecting a clinical site. NPs' lived experiences with the role transition related to preceptorship and sites' matching was explored.

The length of a students' experience at each site is also important for learning. Students' learning can be enhanced by providing longer and uninterrupted clinical experiences avoiding frequent preceptor and site changes. NP students who spend at least three months with one preceptor develop higher self-efficacy levels (Neal, 2008 as cited in Poronsky, 2012). Placing students in one clinical environment for the entire length of their experience may be beneficial in students' understanding of the health care system and developing an effective preceptor-student relationship (AACN, 2015). NP students' fragmented experiences with clinical practicum make students' experiences less meaningful placing an additional burden on clinical sites (Giddens et al., 2014). With frequent preceptor or site changes, preceptors are left to reorient students to the new site, policies, and EMR. Most NP students spend a limited number of hours at multiple sites; lack of familiarity with the sites may impede students' learning. Additionally, for the learning to take place, students must be familiar with the environment, feel safe in it, and be respected and recognized by the health care team (Flott \& Linden, 2016). Quality of learning suffers from great variability and random occurrence of patient care experiences among the sites (LeFlore, \& Thomas, 2016). Instead of tailoring clinical experiences to the students' learning needs, students learn through those experiences that become available to them depending on the setting. Sites that do not offer needed patient diagnoses, the required degree of complexity, and variety or sites that accommodate fewer patients per day may also have a negative effect on students' experiential learning. Establishing a predetermined and sufficient amount of time for students to 
spend at the clinical site may improve students' learning experiences. It was important to explore NPs' experiences with the transition while undergoing the experiential learning at various sites. Precepting accommodations. Providing quality precepting is difficult as preceptors are faced with many barriers. While most skills are developed when NP starts his or her new job (Bazzell \& Dains, 2017), preceptors are a vital part of NP students' clinical preparation for practice (Wiseman, 2013). The most frequently cited barrier to precepting was the amount of time it takes to train (Roberts et al., 2017). Further, preceptors are struggling to accommodate NP students' learning needs due to preceptors' needs to complete their duties of caring for complex patients within the strict time constraints of their schedules (Giddens et al., 2014; Keough et al., 2015; Poronsky, 2012) and increased demands of billing related documentation (Wiseman, 2013). Because preceptors have multiple work-related responsibilities, precepting activities often compete with the time preceptors are expected to complete their work; thus, because employers have control over preceptors' time, the teaching time preceptors may have at work may be limited (Forsberg et al., 2015). In fact, preceptors reported working longer days on the days they trained students to complete their work, complete student's evaluation, and finish work related documentation (Wiseman, 2013).Though the role of preceptors is crucial in NPs' transition, most practices are busy, and patient care takes priority over NP training (Bazzell \& Dains, 2017; Roberts et al., 2017). Adding additional responsibility to a preceptors' already busy day without any adjustment may cause a decrease in preceptor's productivity and satisfaction with their work (Webb et al., 2015). It was important to explore the limitations NPs experienced with their preceptors due to time constrains as well as their effect on NPs' role transition process. 
While lack of time to trainis a critical issue, other barriers also exist to proper precepting of NP students. Such barriers include space limitations, inappropriate settings, and patient volume, as well as student's limited EMR access (Roberts et al., 2017). Limited preceptor's exam room availability may affect the number of patients trainee can see semi-independently. Because students may take a longer time to care for their patients, a provider may have limited space to accommodate patient volume required by his or her organization. Furthermore, the patient volume may not match student's learning needs either overwhelming or underwhelming the student. Additionally, with no EMR access, inability to document on patients may inhibit student's learning progress and increase preceptor's workload. In summary, preceptors have many obstacles to providing quality teaching. If preceptors are unable to overcome these barriers, students suffer; this may translate into poor clinical experiences, which may affect students' role transition.

While NP preceptors' loss in productivity when precepting is still to be addressed, this issue has been dealt with for MDs. After completing 4 years of medical education, a medical student enters four-year medical residency; if one seeks specialty, it may take longer than four years to complete the residency (Dower, 2012). Every year, the Federal government funds approximately 9.5 billion dollars through Medicare and about roughly 2 billion dollars through Medicaid to cover the expense of general medical education (GME) training medical doctors; approximately 100,000 dollars is spent every year on the training of one medical resident (Dower, 2012). It is important to know how other professions support their students in their role transition to appreciate the extent of support available to NP students. More importantly, 
adjusting preceptors' workload to account for precepting duties may play an important role in NP students' and novice NPs' quality of clinical experiences.

Preceptors' training. Preceptors' training is necessary for NPs' quality clinical experience. Though $36.7 \%$ of preceptors expressed desire to be taught to train NP students (Roberts et al., 2017) and preceptors play a key role in NPs' transition, few organizations provide any preceptor training to ensure that NP students and new NPs are supported in their learning of a new role (Bazzell \& Dains, 2017). Approximately 47.3\% of graduate nursing preceptors reported no preparatory work to help them to train students (Donley et al., 2014). Furthermore, no preceptorship guidelines exist for those preceptors who train NPs (Bazzell \& Dain s, 2017). However, structured preceptorship has a positive effect on NPs' clinical reasoning (Bazzell \& Dains, 2017). Thus, guidelines and training for NP preceptors should be considered to improve the process of preceptorship to assist NPs with their role transition.

Precepting graduate nursing students takes special skills. At times, graduate students reportedly have a good social preceptor-student relationship, but only minimal learning occurs; thus, graduate students underlined the importance of having not only a good social but also a professional preceptor-student relationship that would allow for effective learning (Nyhagen, \& Strøm, 2016). To be effective, preceptors need to maintain a trusting preceptor-student relationship; exercise reflective questioning along with using other teaching strategies, as well as to be willing to teach and be confident at their job (Nyhagen, \& Strøm, 2016). It may be more challenging to ensure the effectiveness of precepting graduate students when compared with the undergraduate nursing population. For example, graduate nursing students seemed to have 
occasional disagreements with their preceptors regarding the relevance of the learning cases (Nyhagen, \& Strøm, 2016). This was different from what was learned about the undergraduate nursing students; this difference may be related to that graduate students already have nursing experience compared with the undergraduate nursing students (Nyhagen, \& Strøm, 2016). Graduate students also appreciated when preceptors verbalized their actions during procedures performance (Nyhagen, \& Strøm, 2016). Thus, cognitive preceptorship through offering a detailed rationale for the medical decision-making processes is essential. Moreover, explaining actions requires preceptors to be up to date on current knowledge. However, practical over theoretical knowledge was more emphasized by those preceptors who graduated a long time ago (Nyhagen, \& Strøm, 2016). Therefore, time lapsed from the preceptor's graduation may play a role in knowledge transfer from a preceptor to a student. Overall, graduate students may have learning needs that may be different from those of undergraduate nursing students. NP students may place additional demands of having preceptors who are well trained in their fields and who maintain the up to date theoretical knowledge base.

In addition to the preceptors' abilities to train NPs, awareness of each other level of expertise and expectations is important for the successful preceptor-trainee relationship. Thus, to provide effective precepting, preceptors need to be aware of trainees' degree of advancement. It is important for the preceptor to evaluate the trainee's knowledge and the level of independence (Babcock et al., 2014). Precepting NPs should include preceptor's orientation to the trainee's course syllabus, objectives, and expectations (Babcock et al., 2014). Precepting NPs is a complex process. NP role functions of diagnosing and prescribing are complex and require trainees think 
critically. Graduate students and novice NPs expect to be taught to problem solve independently and not to simply follow their preceptors' directions (Nyhagen, \& Strøm, 2016). Such instruction requires close communication between the preceptor and trainee. Thus, the approachability of a preceptor is one of the important factors in successful preceptorship (Flott \& Linden, 2016). Lack of understanding of each other's expectations forms a barrier to effective preceptorship (Nyhagen \&Strøm, 2016; Rhrich et al., as cited in Poronsky, 2012). Preceptors' knowledge of their students' abilities and expectations along with trainees' familiarity with their preceptors' expectations and expertise level are the important factors to consider when examining the role of preceptorship in NP students and novice NPs' role transition.

In addition to NP preceptors, other professionals may be involved in NPs' training. NP preceptors may include NPs, MDs, PAs, and DOs (Webb et al., 2015). Interdisciplinary learning is becoming more popular in current healthcare education. Because in real life the quality patient care is an outcome of interdisciplinary work, it is important for different professionals to work and train together to enhance interdisciplinary practice and; thus, increase patient satisfaction and quality of patient care (Chen et al., 2016). Because NPs may be trained by other professionals, it is important to educate other professionals about the specifics of NP clinical education.

However, no formal training on interdisciplinary preceptorship was offered to help preceptors in training other professionals (Chen et al., 2016). But the need for such training exists. When training those from other professions, preceptors expressed the lack of knowledge about their role, knowledge, and training level, as well as the scope of practice (Chen et al., 2016). Without such training, it is hard to tailor clinical experiences to trainees' learning needs, which renders 
NP clinical education potentially ineffective. Though there are many differences among the professionals' training programs and scope of practice, most preceptors used the same teaching methods for all students (Chen et al., 2016). However, the same teaching methods may not be appropriate for various levels of students' preparatory levels. NP trainees' clinical experiences' value may be decreased because of preceptors' inability to accommodate students' learning needs. In fact, because of billing rules that limit formal supervision of students from other professions, and the need for MD preceptors to review their students' charting with many NP students having no EMR access, some preceptors had concerns about being unable to provide for interdisciplinary training (Chen et al., 2016). Moreover, because of variances between different professions schools' schedules, NP students may have different start times, which cause them to occasionally miss the clinical orientation (Chen et al., 2016). However, becoming familiar with the clinical site and preceptor is an important part of student's learning experience. Thus, though various professionals are involved in training NPs, they often are not familiar with the learning needs of NPs. Learning about NPs' experiences with different types of preceptors and how these experiences affected their role transition was essential in gaining an understanding of NPs' transition to practice process. In summary, preceptor training is rarely offered. However, need for preceptor training exists. Such training would increase preceptors' sensitivity to students' learning needs.

Preceptors' motivation. The precepting role is vital to NP trainees' learning, and most preceptors are willing to invest in future NPs. In fact, $84 \%$ of graduate nursing preceptors expressed a desire to train two or more graduate students (Donley et al., 2014). However, using 
motivation and incentives is important to find and secure preceptors and clinical sites in today's competitive environment. Preceptors are more willing to train if they feel motivated, especially in the form of their organization's support (Bazzell \& Dains, 2017). Most frequently noted incentives included credit toward the recertification, financial compensation, and access to clinical resources (Webb et al., 2015). Thus, both monetary and nonmonetary types of compensation should be provided to reimburse preceptors for their time and effort. Though the compensation of preceptors is necessary to ensure the availability of clinical preceptors and sites, it is not always possible to allocate such support. While medical residents' education is supported by Medicare reimbursement, advanced nursing programs rely on students' tuition and scholarships (Donley et al., 2014). Thus, while precepting is central for students' role transition, few opportunities currently exist to compensate preceptors.

Without proper compensation, it may be more challenging to secure preceptors and sites. However, the majority of preceptors reported never having any monetary compensation for precepting (Wiseman, 2013). Financial compensation of clinical preceptors is minimal with only 4\% of NP programs offering this type of compensation (AACN, 2015; Erikson et al., 2014).

However, "payment incentives for community-based sites were used by $71 \%$ of D.O.

Participants, 20\% of P.A. Participants, and 15\% of M.D. Participants” (Erikson et al., 2014, p.3). Without proper compensation for preceptor's work, preceptor fatigue is likely to occur; preceptors may choose to stop precepting (Forsberg et al., 2015).With other professions utilizing monetary compensation for their students' precepting more frequently, NP programs may experience more difficulties as competition for preceptors and sites increases. 
Non-monetary compensation is also important in reimbursing clinical preceptors. Organization's involvement becomes central in motivating preceptors. However, preceptors did not report that their organizations provided rewards such as career advancement and such because of their precepting activities (Donley et al., 2014). Precepting is a labor-intense process, and it requires recognition. While preceptor employer's encouragement is an important motivator, an involvement of academia is also necessary. Non-monetary sites' compensation by the nursing programs included university-based positions, access to the library resources, and professional development incentives (Erikson et al., 2014). MD, DO, and PA schools offered preceptors faculty positions in $86,97 \%$, and $58 \%$ of cases respectively, NP schools motivated preceptors by offering them faculty positions in $34 \%$ of cases (Erikson et al., 2014). Similarly, library access was granted to 85,87 , and $66 \%$ of clinical preceptors hired by MD, DO, and PA schools respectively (Erikson et al., 2014). However, only 43\% of NP schools offered library access to their preceptors (Erikson et al., 2014). Integrating the preceptors' role with the organizational goals, as well as having universities to provide preceptors with various forms of compensation for precepting, is necessary. It was useful to explore if NPs had to "settle" for less ideal sites or preceptors because of inability to find or secure clinics; and if so, how this “settling” affected an NPs' transition experience.

Electronic resources. While clinical preceptors and sites constitute the main structure of students' clinical support necessary for NP students to learn their new role, in today's fast pace health care environment students often rely on their electronic resources to both document and retrieve evidence-based resources. Documentation, billing, and coding were identified as weaker 
areas during the NPs' transition (Dillon, 2016). Moreover, most academic leaders reported concerns about students' EMR access and training (AACN, 2015; Erikson et al., 2014). With each student log in cost reaching several thousand dollars (Forsberg et al., 2015), many NP students were left without having an opportunity to chart or use computers to enhance their learning. Even without their own EMR access, NP students used the help of their preceptors to look up patient information to be able to care for their patients. But, without prior EMR training, students were slowed down by an unfamiliar interface of the EMR. Providing student with EMR training prior the practicum start date decreases the number of clinical hours lost on such training during the practicum (Babcock et al., 2014). In addition, because one of the foundational NP skills is students' utilization of evidence (Graue et al., 2015), inability to access the needed resources because of not having a login, may inhibit students' ability to learn effectively. Further, not being able to document on their patients, NP students' communication with their preceptors may be inhibited decreasing the amount of feedback students may obtain from their preceptors. However, preceptors' feedback is essential to NPs' successful role transition.

Overall, the way NP students' role transition is supported during school is important in how students will transition after their graduation. More specifically, clinical preparation is central to students' acquiring new NP skills necessary for them to function in a new role. With the lack of availability of clinical sites and preceptors, variable levels of students' preparation for their clinical experiences; preceptors' lack of training in teaching, time, and compensation for precepting, along with mismatched preceptor-trainee relationships, it was necessary to explore NPs' experiences with the role transition while in a preceptorship. 
Post-graduation resources. While the main way of learning clinical skills during the pregraduation role transition stage is through the students' relationships with their preceptors and clinical sites, the way NPs' transition stage is supported after graduation involves more variation. During this transition stage, NPs' support, if offered, may include collaborative physicians, assigned or formal preceptors, informal preceptors, training on the job, or post-graduation residency programs.

Post-graduation residency programs. NPs' role transition occurs over time starting with RNs entering NP programs and ending years after graduation. Moving from a more structured environment of higher education to the work environment signifies a change in expectations and responsibilities (Lindfors et al., 2014). Post-graduation residency programs are an effective way of closing the gap between academia and practice helping novice NPs to transition to their new role successfully.

Many professions, including medical, provide well-organized and meticulously structured post-graduation residency programs to help their new graduates with the transition to practice process after graduation. For instance, the American Osteopathic Association program had 548 graduates who completed the post-graduation residency program in family and internal medicine in 2015 (American Association of Colleges of Osteopathic Medicine, 2016). Similarly, there were 1,994 pharmacy post-graduation residency programs in the United States that had 3,987 open residency positions with one pharmacy residency program being available per each 7 to 8 pharmacy newly graduated applicants (American Society of Health-System Pharmacists, 2016). The demand for these types of programs is high (American Society of Health-System 
Pharmacists, 2016). Likewise, there were 215 physical therapy post-graduation programs in the country (American Physical Therapy Association, 2016). Furthermore, for registered nurses $(\mathrm{RN})$, there were 187 practice sites in 32 states that have provided the opportunity for 40,000 RNs to transition from school to practice while significantly increasing RNs' retention rates at the same time (American Association of Colleges of Nursing, 2016). Overall, many professions consider well-established post-graduation residency programs necessary for their new graduates' success and retention in the profession.

While the aforementioned professions provided much-needed support to their new graduates, the advanced nursing profession lags behind in supporting novice NPs. Consequently, the NPs' transition process from academia to practice after graduation is unpredictable and lacks support (Flinter \& Hart, 2016). Some argued that NP residency programs are not needed because NPs receive an adequate masters-level education (Sroczynski, \& Dunphy, 2012). However, Chappell (2014) noted that NPs have similar to other professionals needs for robust support of their transition to practice.

There is a gap in how the transition to practice is regarded by the nurse practitioner profession and other medical professions. With about $90 \%$ of NPs expressed an interest in postgraduation residency programs (Hart \& Bowen, 2016), in 2016, only 37 nurse practitioner primary care post-graduation training programs were available across the United States in 2016; "23 of the programs were in federally qualified health centers (FQHC), 7 were in the VA system, 3 were in hospital/health systems, and 4 were in non-FQHC clinics” (M. Flinter, personal communication, June 8, 2016). Furthermore, each program was limited to admitting 
approximately 3 to 6 NP graduates, graduating approximately a total of 150 NPs a year (M. Flinter, personal communication, June 8, 2016). With 23,000 NPs graduated in the years of 2015-2016 (AANP, 2017a) and 37 NP post-graduation programs available (M. Flinter, personal communication, June 8, 2016), one such program was available per $621 \mathrm{NP}$ graduates. The difference in the availability of role transition assistance between the NP profession and other professions is significant. Because the post-graduation programs are not yet widely available to the newly graduated NPs, to understand NPs' role transition process in the current environment, it is important to study currently available resources used to support most novice NPs in their role transition. More specifically, exploring NPs' experiences with their role transition along with the support offered through preceptorship after their graduation was needed to understand their transition to practice process.

Preceptorship through collaboration. Novice NPs' post-graduation support is limited. Formal preceptorship is uncommon (Wilkes \& Feldman, 2017) with only 17\% of novice NPs reporting having a formal preceptor during their role transition at work (Hart \& Bowen 2016). Though formal preceptorship after graduation is infrequent, most NPs expressed the desire for such support by indicating the need for post-graduate residency programs to assist with their transition (Hart \& Bowen, 2016; Jones et al., 2015). When preceptors are assigned, small numbers of NPs' preceptors is represented by experienced NPs (Jones et al., 2015). With the lack of formal preceptorship to support novice NPs' role transition after graduation, the major source of preceptorship for NPs becomes through having a formal collaborative agreement with their assigned physicians; thus, most NPs' preceptors are MDs (Jones et al., 2015). Learning from 
other professionals is an important attribute of NPs' successful transition to practice (Faraz, 2016; Farrell, Payne, \& Heye, 2015). Most novice NPs must have a collaborative agreement with MDs and rely on MDs' support; however, MDs' abilities to train may be limited.

Medical and nursing paradigms differ significantly and yet, MDs are expected to guide NPs in their role transition. Misunderstandings about the NP role and scope of practice appear to be a barrier to their role acceptance (Lipley, 2014). Physicians' knowledge of NPs' role and scope of practice is important for these professionals' effective collaboration. However, MDs have little familiarity with NPs' role, education, and training (Schadewaldt et al., 2013; Van der Biezen et al., 2017), which is the most common barrier to a successful NP and MD collaboration (Schadewaldt et al.,2013). Physicians' lack of clarity about NPs' profession and the scope of practice may inhibit NPs' ability to have a meaningful preceptorship after graduation.

Conflict of interests may also diminish the ability of MDs to support novice NPs effectively. Besides not being familiar with NPs' role, novice NPs' collaborators often combine the responsibilities of providing NPs with advice with the simultaneous evaluation of NPs' job performance (Poronsky, 2012). Thus, MDs are often NPs' employers, and they expect NPs to bring profit to their practices. Thus, instead of having novice NPs' successful transition at heart, MDs' priority often becomes the profit NPs bring to the practices. Hiring NPs is a cost-effective way of providing primary care that leads to high patient satisfaction scores (Hevesy et al., 2016). Though MDs expect to gain financially from hiring NPs, they are also expected to provide NPs with support and training during NPs' transition from school to practice. NPs' transition to practice is then affected by the gap between the employers' expectations and newly graduated 
NPs' entry preparation level (Andregard \& Jangland, 2014; Sargent \& Olmedo, 2013). Thus, NP dependency on his or her collaborator physician right after graduation may create an unintended conflict based on unmet and unrealistic physician's expectations and their lack of knowledge about NPs' learning needs after graduation. Exploring NPs' experiences with their assigned collaborator MDs during their transition to practice illuminated on the reasons why NPs' transition process is difficult.

While MDs are the first line of support for novice NPs, patients and RNs may also play a role in aiding NPs' transition. However, physicians are not the only ones who lack understanding about the NP role. Often patients and RNs do not have the full understanding of an NP role. Relationships with physicians, nurses, and patients alter after an RN transitions to her or his new NP role (Maten-Speksnijder et al., 2015). RNs may lack understanding of why NPs can prescribe and give them orders while patients may expect NPs act the same as their MDs or their nurses. The tension created by the lack of NP role understanding by RNs and patients may affect novice NPs' learning after graduation.

While confusion about NP role and frequent conflict of interest may be restraining MDNP collaboration, the traditional hierarchical relationship between MDs and NPs may be another impeding factor of their collaboration. Collaborations among the professionals are not always effective due to different views on traditional hierarchy (MacNaughton, Chreim, \& Bourgeault, 2013). Though collaboration and learning are expected to occur during the NPs' transition to practice process; the prescriptive and hierarchical nature of the MD-NP relationship may make NPs' preceptorship and transition process more difficult. At the same time, when well supported, 
collaboration can greatly improve NPs' transition to practice process (Dillon, 2016; Faraz, 2016). In fact, collegial support from physicians and leaders, when effective, was found to be a significant NP transition facilitator (Maten-Speksnijder et al., 2015). If successful, such collaboration can benefit NPs and patients. As a result, the ability to collaborate with other professionals can increase the quality of care patients receive (Andregard \& Jangland, 2014). With the traditional hierarchy in the way; however, when working together, MDs often expect NPs adjust to MDs' collaboration style (O'Brien et al., 2009). But MDs are expected to teach and support novice NPs through collaborating with them; precepting assumes allowing the trainee to become stronger and more autonomous in his or her decision making and problem-solving skills (MacNaughton et al., 2013). Conversely, instead, MDs may be more inclined to advise NPs to follow MDs' protocols undermining novice NPs' autonomy. This may be because MDs have concerns regarding collaboration with NPs, which include NPs' education, competence, limitations in the scope of practice, as well as MDs' liability for NPs' actions (Schadewaldt et al., 2013). Thus, it was important to gain a deeper understanding of the role of such hierarchical collaboration has in NPs' preceptorship and NPs' role transition.

While precepting by collaborator MDs is important to NPs' role transition, gaining autonomy is also central to NPs' transition when taking on new responsibilities of diagnosing and prescribing (Barnes, 2015b). Whereas many NPs seek a collaborative relationship with MDs, many would also like their autonomy of practice to be respected (Schadewaldt et al., 2013). Thus, though working in a team is essential, it is also important to consider role boundaries and each role-specific function for the collaboration to be successful (MacNaughton et al., 2013). In 
professional practice, both autonomy and collaboration are important for the professional role development and for the team members to work successfully together (MacNaughton et al., 2013). Defining role boundaries can facilitate successful collaboration among the different professionals through considering each professional's workload, physical space, leadership, education, as well as individual's attitudes and values (MacNaughton et al., 2013). Therefore, autonomy may be an important component of healthy collaboration within the team; person's autonomy allows one to feel that their contribution is respected (MacNaughton et al., 2013). Respecting novice NPs' autonomy by providing them with the opportunity to problem solve while supporting them with advice instead of expecting NPs to abide by MDs' protocols is an important aspect that must be considered if this collaborative relationship is to be effective in precepting novice NPs.

Though the precepting relationship between novice NPs and MDs may be affected by lack of MDs' knowledge of NPs' role and their traditional hierarchical approach to collaboration with nurses, this relationship may be the only source of preceptorship support novice NP has after his or her graduation. In addition to aforementioned issues effecting novice NPs' postgraduation preceptorship, MD-NP collaboration often occurs remotely, further impeding novice NPs' ability to adjust to their new role. However, face-to-face communication was found to be imperative in creating a good collaborative relationship between NPs and MDs (Schadewaldt et al., 2013). Additionally, nurses and physicians collaborate better in high acuity smaller areas (O'Brien et al., 2009). Further, it normally takes about three to six months to develop a good collaborative relationship (Schadewaldt et al., 2013); time spent on precepting is critical for a 
preceptor-trainee relationship (Poronsky, 2012). However, the first six months may be the most critical time in NPs' transition to practice process. Not yet having a well-developed collaborative relationship combined with the inability to have MD on site for necessary face-to-face consultations regarding variations in patients' presentations may negatively affect some NPs' role transition and force NPs to resign or seek informal preceptors. Thus, it was important to explore the role collaboration with MDs has in NPs' role transition.

Overall, the two concepts, transition and preceptorship have limited research available in relation to the NP population. It is known that NPs have significant difficulties with transitioning to their new role (Brown \& Olshansky, 1997; Flinter \& Hart, 2016; Steiner et al., 2008). However, most studies completed to date were focused on NPs' role transition after graduation (Brown \& Olshansky, 1997; Chappell, 2014; Flinter \& Hart, 2016) but as described in earlier studies (Heitz et al., 2004; Steiner et al., 2008), the role transition process starts long before the graduation. Thus, holistic approach to investigating the concept of role transition is necessary to understand NPs' transition during the time before and after graduation. Additionally, many scholars voiced concerns regarding the clinical training and preceptorship, which support NPs' transition to practice process; they revealed that the current state of clinical education and preceptorship is in need of improvement (AACN, 2015; Brown \& Olshansky, 1997; Donley, et al., 2014; Flinter \& Hart, 2016; Hallas, et al., 2012; Logan et al., 2015; Roberts et al., 2017; Steiner et al., 2008; Sullivan-Bentz et al., 2010; Webb et al., 2015). In spite of the insights gained from these studies, policies supporting NPs' transition through preceptorship are yet to be developed. 
This literature review showed that the quantitative methods have been helpful in showing the relationship between formal training and NPs' role transition (Barnes, 2015b) and that NPs' retention may be affected by their difficult transition process (AACN, 2015). However, the qualitative approach was chosen for this study to identify those elements of transition process and preceptorship that have not been uncovered yet. Identifying these important elements illuminated additional areas within transition and preceptorship that can be better supported through new and improved policies. Thus, the qualitative approach has been chosen in this study to understand NPs' experiences with the transition while guided by preceptors.

\section{Summary}

Research has provided consistent indication that a profound need exists for an increased support of NPs' role transition, both before and after graduation. NPs' clinical experience is at the core of their learning their new NP skills. Competing with many other schools, NP students and their faculty struggle to find preceptors and sites to ensure quality clinical experiences. At the same time, NP students' preceptors struggle to incorporate NP students' teaching into their already busy schedules, risking their employment because of their decreased productivity while teaching. NPs graduate with entry-level preparation lacking some important skills such as suturing or radiographic interpretation, for example. MDs being unfamiliar with NPs' education and their post-graduation learning needs, expect NPs to fully function in today's complex health care environment right after graduation. Most novice NPs do not have formal preceptors assigned; thus, they rely on MDs to support them. However, literature showed that such preceptorship might be insufficient since MDs are unfamiliar with NPs' educational background, 
they may have a conflict of interest, and they may expect the traditional hierarchical approach to precepting when working with advanced nurses. NPs' role transition is found to be very stressful with many NPs leaving their first NP job during their first year of practice.

From a scarce number of studies available on NPs' transition to practice, the focus was on NPs' post-graduation transition experience. Additionally, researchers emphasized and studied post-graduation residency programs. However, most novice NPs do not have an opportunity to attend such program with only few NP residencies available across the country. It becomes important to study NPs' experiences with those transition support systems that are available to most novice NPs, including preceptorship. While some information was available on NPs' experiences with the role transition, it was not known how well current preceptorship supported NPs' role transition process. This study filled the gap in knowledge by studying NPs' experiences with their role transition while being in preceptorship before and after graduation. Because NPs' role transition is a wide area that needs exploration, this research was focused on a discrete component of transition related to preceptorship as preceptorship was indicated to be in great need of restructuring through this literature review. This study results provided insights to academic leaders, organizations' leaders, policy makers, NP preceptors, NP students, and novice NPs on the aspects of NPs' transition support related to preceptorship. The results of this study may have offered solutions on how to maximize those transition resources that are currently available. Because little information was available on the two concepts of interest, a qualitative approach was necessary to begin studying these concepts in depth. Chapter three addresses the methodology of my study. 


\section{Chapter 3: Research Method}

\section{Introduction}

The purpose of this hermeneutic phenomenological study was to understand and describe the meaning of NPs' role transition experiences while in a preceptorship before and after graduation. In Chapter 3, I present a thorough description of the research methods, which consists of the research approach with the rationale; my role as a researcher in this study; a description of study's population; the interview protocol and its justification; the procedures for sampling, recruitment, data collection, and data analysis; justification of the study's trustworthiness; and ethical procedures.

\section{Research Design and Rationale}

The research question posed in this study was as follows: What was the experience of transition like for an RN becoming an NP under the guidance of a preceptor during their academic program and after graduation? The central phenomenon of interest in this study was NPs' role transition while under the preceptor's guidance. NP role transition is defined as a shift from being a licensed RN to becoming an NP (Barnes, 2015b). During this shift, an individual undergoes an extensive learning of a new role, assumes new responsibilities and expands professional autonomy while struggling to reconcile a new professional identity of the two roles, a nurse and a provider (Barnes, 2015). The process of NP role transition is also characterized by “mixed emotions" (Barnes, 2015b, p. 139). Additionally, preceptorship is defined as a guiding and supportive, one-on-one relationship between the trainee and an expert, both before (Kelly \& Mcallister, 2013) and after graduation (Korzon \& Trimmer, 2015). 


\section{Philosophical Assumptions and Research Design}

To explain why a certain research design was chosen, it is important to understand the philosophical assumptions of the researcher (Creswell, 2013). A researcher's beliefs have a profound effect on every aspect of the study (Creswell, 2013). A researcher's paradigm is based on assumptions about reality and beliefs about the phenomena (Maxwell, 2013). Therefore, examination of one's philosophical assumptions is important to ensure alignment within the study (Lopez \& Willis, 2004). Methodology and results may suffer when there is misalignment between the researcher's assumptions and the research approach.

Researchers' ideas about reality and the ways of learning about that reality are grouped into four categories or four philosophical assumptions, which become instrumental in any research study. : According to Creswell (2013), these include ontological assumption (beliefs about the reality), epistemological (beliefs about the knowledge), axiological (beliefs about values), and methodological (beliefs about the research process) Clarifying philosophical assumptions about how the study is based helps understand how the knowledge was produced (Norlyk \& Harder, 2010). Based on my beliefs about reality and knowledge, I considered my philosophical paradigm be consistent with critical realism. Multiple forms of realism, including critical realism, provide a ground on which qualitative research can be built (Patton, 2015). Consistent with critical realism, I believe that while objective reality exists separately from the individuals, their experiences, genetic makeup, and environment affect their perception of reality (Maxwell, 2013). 
Further, critical realism includes the consideration of objective reality together with the context. Thus, "realism emphasizes that truth is context dependent" (Patton, 2015, p. 111). People's perceptions are affected by their beliefs and experiences, as well as by the reality they come in contact with as they move through their lives (Maxwell, 2013). Further, critical realism is based on the understanding that the reality exists independently of one's conciseness; however, the reality constructed by people also must be considered and explored (Patton, 2015). Thus, I believe in the importance of multiple realities within a specific context; these realities are constructed by individual experiences and examined with the use of multiple research tools (Creswell, 2013). This belief was important in my phenomenological study as I planned to collect data about my participants' lived experiences extracting the essences and meanings of their experiences with the phenomenon. It is also important to note that realists view unobservable phenomena as real (Patton, 2015), emphasizing the importance of the role of the individuals in constructing the reality (Mark et al., 2000, as cited in Patton, 2015). This made the analysis of my participants' experiences with the phenomenon relevant to my research question and design choice. Thus, the critical realist believes that although the world is a separate reality, individuals' constructions of the world are critical and need to be examined (Patton, 2015). Understanding researcher's philosophical paradigm assists in justifying various choices made by the researcher while conducting a study.

\section{Qualitative Nature of the Study}

While my philosophical assumptions influenced my choice of the research design, another reason for choosing qualitative approach was the lack of research for NP population role 
transition. Thus, qualitative research approach has been chosen to hear the voices of my participants within my study's context to capture the uniqueness of my participants' responses and to explore the issue of interest in great detail. A qualitative research design was a good fit for my study's purpose as it allowed me to conduct research in the presence of natural setting considering context, use a researcher as the key instrument, apply multiple data collection procedures, as well as understand and interpret the participants' meanings (Creswell, 2009). Qualitative studies also allow the researcher to choose depth over breadth to explore the phenomenon of interest (Patton, 2015). Therefore, having an opportunity to interview my participants for a prolong time allowed me to collect qualitative detailed data. In addition to choosing a qualitative design, I made a decision on the type of a qualitative design necessary to answer my research question.

\section{Phenomenology}

There are various approaches of inquiry that the researcher can choose from when conducting a qualitative study. Selecting the most suitable research approach prior to starting a study is critically important for the study's effectiveness (Creswell, 2013). Although all qualitative approaches share many similarities related to the general research process, there are noticeable differences among the approaches, especially when it relates to the type of data received in a study (Creswell, 2013). Thus, the ways of collecting and analyzing data differ significantly among the qualitative research approaches (Creswell, 2013). When considering differences and similarities among the qualitative research designs, it was of the most significance to evaluate the chosen design's alignment with the study. The degree of design 
alignment with the research study translated into the quality of data received, which then ensured the correctness of the results. Thus, the design should be chosen based on the type of the research problem, studied unit of analysis, data collection and analysis techniques, and the study's general structure and focus (Creswell, 2013). However, most importantly, the research design choice must be guided by the research question (Van Manen, 1990). I wanted to answer the question about my participants' lived experiences with the role transition while in a preceptorship; thus, I have chosen the phenomenological research approach.

The phenomenology design fits well with the aim of my study. Additionally, phenomenology research approach is frequently used in nursing (Dowling, 2007).

Phenomenology means "'to the things themselves", and "let's get down to what matters!' (Van Mannen, 1990, p. 184). Considering the design's characteristics is important when choosing a research approach. More specifically, it is important to note that phenomenology's focus is on the phenomenon itself rather than on the individual who experiences it (Van Manen, 2017). To allow for study usability, it was important to clearly discuss study methodology providing for maximum transparency of choices made regarding the methodology (Norlyk \& Harder, 2010). Thus, discussing the chosen design in detail was necessary.

Phenomenology encompasses both the philosophy and methods (Heinonen, 2015). It is vital to understand the differences between phenomenology as philosophy and phenomenology as a method (Dowling, 2007). In other words, methodology must be distinguished from methods as methodology is the philosophy behind the methods or techniques (Van Manen, 1990). For example, using interview procedure for data collection or sample selection procedure refers to 
methods (Van Manen, 1990). When speaking of phenomenology as philosophy, it is important to note that the purpose of phenomenology is to reduce participants' experiences with the phenomenon to the essences or meanings of the phenomenon to bring the phenomenon to a clear view (Van Manen, 1990). Thus, phenomenology assists in identifying common experiences related to the phenomenon of interest (Creswell, Hanson, Clark Plano, \& Morales, 2007). Different perspectives exist on how to approach phenomenology.

A phenomenological approach can be descriptive or interpretive (Tuohy, Cooney, Dowling, Murphy, \& Sixsmith, 2013; Welford, Murphy, \& Casey, 2012). Descriptive phenomenology allows one to focus on describing the phenomenon's main attributes, while an interpretive phenomenology approach also includes an interpretation of the persons' experiences (Tuohy et al., 2013). Because there are many types of phenomenology and some may contradict each other, it is essential to draw from one chosen approach when guiding study's methodology (Patton, 2015). Notably, all phenomenological approaches share common assumptions of studying lived human experiences with the phenomenon of interest while capturing the essences of such experiences with the phenomenon (Creswell, 2013). While different phenomenological perspectives have common fundamental assumptions, they also have some differences. Edmund Husserl wrote substantially on descriptive phenomenology emphasizing the need for the researcher to completely disassociate from their own experiences when performing research (Creswell et al., 2007). Further, Husserl's understanding of phenomenology was to uncover the essence of the phenomenon free of bias using phenomenological reduction without any interpretation (Dowling, 2007). Thus, Husserl taught to describe the experiences before they 
have been reflected on (Dowling, 2007). It is also important to understand that with Husserl's view on phenomenology, researchers focus on the phenomenon itself but not on participants' interpretation of the phenomenon (Dowling, 2007). Thus, two assumptions of Husserl's approach to phenomenology include bracketing (minimizing bias and any preexisting knowledge of the phenomenon) and recognizing that in all human living experiences there are some common attributes applicable to all people (Lopez \& Willis, 2004). The assumption that context does not need to be considered in understanding the essence of human experiences is also one of the most important characteristics of Husserl's approach to phenomenology (Lopez \& Willis, 2004). Thus, Husserl's phenomenological perspective excludes the context or situation of those who experience the phenomenon of interest.

Heidegger expanded on Husserl's work by implementing interpretive phenomenology (Lopez \& Willis, 2004). While Heidegger agreed with Husserl that phenomenology is concerned with human experiences, he completely opposed positivism, entirely relying on interpretation (Dowling, 2007). In contrary to Husserl's, Heidegger (a student of Husserl) taught the use of hermeneutics (interpretation) in phenomenology (Rachers, 2003, as cited in Dowling, 2007). The context of the phenomenon is important when hermeneutics are applied to one's research (Lopez \& Willis, 2004). Contrary to Husserl's teaching, in Heidegger's approach, a researcher's experience is a valuable tool used to interpret study findings (Lopez \& Willis, 2004). The main focus of phenomenology is to explore lived human experiences while identifying the essence of the studied phenomenon (Ajjawi \& Higgs, 2007). With the application of hermeneutics, researchers can apply their knowledge to interpret participants' experiences (Ajjawi \& Higgs, 
2007). In other words, phenomenology allows describing, and hermeneutics allow extracting the meanings of the experiences with the phenomenon (Van Manen, 1990). Thus, to explore the common essences of participants' experiences, the researcher uses his or her knowledge and experience.

This hermeneutic phenomenological study is guided by the work of Van Manen, whose approach combines both descriptive and interpretive methodology to phenomenology (Dowling, 2007; Van Manen, 1990). Max Van Manen is a Canadian phenomenologist; his work has become popular in a medical field (Ajjawi \& Higgs, 2007; Mak \& Elwyn, 2003 as cited in Dowling, 2007). According to Van Manen (1990), both description and interpretation of hermeneutic phenomenology are intimately connected to the situation or a context. One of the aims of human science is to explain "the meaning of human phenomena" and to understand "the lived structures of meanings" (Van Manen, 1990, p.4). Thus, the preferred methods to study human sciences include "description, interpretation, and self-reflective analysis" (Van Manen, 1990, p.4). Van Manen (1990) referred to the description as being interpretive when the description points to the meanings of the experience. Though using pure description is important to uncover the important attributes of the studied phenomena, exploring the contextual attributes of participants' experiences is also important for study's practical application. When used together, the descriptive and interpretive approaches allow for a deeper understanding of the participants' experiences (Matua \& Van Der Wal, 2015). Capturing the description of experiences along with the context can make the study results more applicable to various contexts and situations where processes need to be improved. 
Van Manen's use of interpretation when extracting the meanings of the studied experiences with the phenomenon increases this method's usability by researchers when context is of an importance. Van Manen's methods are used widely among nurses who seek to use the phenomenological approach in their studies (Heinonen, 2015). Hermeneutic approach assists in capturing the meaningful interpretation of the experience allowing for grasping its significance (Van Manen, 1990). Husserl's tendency to objectify the phenomenology method may cause one to ignore the content in which phenomenon occurs (Van Manen, 1990). The preferred method to study natural or physical sciences is through observation and experience; however, studying human sciences requires to take into consideration the meanings people create to act and express themselves (Van Manen, 1990). Phenomenology allows to capture these meanings of experiences humans have (Van Manen, 1990).

Van Manen's (1990) approach is based on the principle that while it is necessary to approach participants' experiences with openness through reduction or epoche, it is not realistic for the researcher to completely separate and eliminate own experiences from the research. Thus, instead of trying to eliminate researcher's bias, Van Manen teaches to focus on acknowledging how this bias might influence the research (Van Manen, 1990). Phenomenology allows for deep understanding of the everyday experiences as they occur before one has an opportunity to reflect (Van Manen, 1990). However, one's awareness determines what experiences one will describe (Van Manen, 1990). Thus, one's consciousness plays a significant role in what experiences are included in one's descriptions (Van Manen, 1990). Additionally, it is not possible for the participants to produce a pure description of their experiences as what is described has already 
been processed and chosen for description by the participants; thus, introducing interpretation into the research (Van Manen, 1990). Van Manen's approach to phenomenology helped me to stay open to emergent data while acknowledging my previous experience with the phenomenon. By using this approach, I was better equipped to collect pre-reflective descriptive data from my participants as recommended by Van Manen (1990). This high-quality descriptive data was then carefully reflected on by me to gain deep understanding of the phenomenon I was studying.

\section{Role of the Researcher}

I carried out the entire research process including data collection, analysis, and reporting of my study results. My role in this study also included recruiting study participants, obtaining informed consent, and conducting semi-structured audiotaped interviews. I had no personal, professional, or power relationship with my participants. I excluded any of my students or friends from my research to avoid bias. Additionally, I used a 10 dollar Amazon certificate per each participant to compensate my participants for the time spent on the lengthy interviews.

\section{Researcher's Bias}

To avoid bias in a research study, it is important to understand the meaning of bias in qualitative research, acknowledge potential sources of bias, and describe how they will be managed. To approach bias in the research, it is important to note that while biases are always the source of an error in quantitative research that needs to be eradicated, in qualitative research the approach to minimizing bias is through understanding and "visualizing" those areas of research that could potentially be affected by bias (Roulston \& Shelton, 2015). In other words, in qualitative studies, instead of eliminating researcher's perceptions, scholars should strive to 
explain how their assumptions have affected the study (Maxwell, 2013). To counteract the possibility of assigning specific meaning to participants' statements, reflexivity was used (Janesick, 2011; Van Manen, 1990). I exercised reflexivity through self-reflection on my experiences and assumptions. Reflexivity is when one understands one's role in relation to others (Tsekeris \& Katrivesis, 2008). Describing my role and the role of my experiences in relation to my study was necessary to produce quality results.

Bias can come from both personal experiences and literature review. In this study, I am both the researcher and the representative of the same profession as my participants. More specifically, I had experienced NP role transition as well as functioning in a role of an NP student, NP novice, NP expert, NP preceptor, and NP faculty. Awareness of my own experiences played a role in choosing the phenomenon of study (Van Manen, 1990). By discussing the reason behind the choice of my research topic, I increased my study’s objectivity (Roulston \& Shelton, 2015). My research interest in NP role transition while in a preceptorship has been prompted by my personal experiences with my NP role transition and the difficulties that both I and my NP students had experienced with their preceptorship over the past ten years. Because it is important to recognize researcher's assumptions to minimize bias (Roulston \& Shelton, 2015), in my research, I ensured that my assumption of difficult role transition and preceptorship did not penetrate my data collection and analysis as I realized that NPs may have different experiences with the same phenomenon.

While my closeness to my participants' experiences was the potential source of bias, it was also one of my study's strengths. Through such familiarity with the profession may have 
introduced some bias into my research, belonging to the same profession may have benefited the study because it may have been easier for me to gain the trust of my participants as well as communicate with them using the familiar terminology used within the profession (Ajjawi \& Higgs, 2007). Thus, my personal experiences with the phenomenon of this study were carefully examined during each stage of this research process.

The literature review process can also be a source of bias. However, reviewing other scholars' work related to the phenomenon of interest may assist the researcher to try to explore the phenomenon the way it has not been looked at before (Van Manen, 1990). Additionally, reviewing literature is considered a source of data as it allows a researcher to develop more insights into his or her own approach to a phenomenon (Van Manen, 1990). Therefore, in my study, my transparency about the reviewed literature assisted with avoiding bias. More specifically, my theory choices, as well as reviewed studies could all be a source of bias. My thorough explanation of the role of theory and various related studies in my phenomenon of interest assisted in identifying their influences on my research.

To further exercise reflexivity, I used journal writing. Because in qualitative studies "the researcher is the research instrument," journal writing assists in self-reflection allowing the researcher to critically evaluate their own interpretations (Janesick, 2011, p.156). Journal writing was used in all stages of my research to help focus my study, assist with data analysis, and clarify thoughts (Janesick, 2011). Thus, journal writing assisted in separating my views and interpretations from the data. 
In addition to journal writing, I used field notes to help to minimize bias by improving data accuracy. I used three types of field notes, which consisted of a transcript, personal, and analytical notes (Minichiello, Aroni, Timewell, \& Alexander, as cited in Ajjawi \& Higgs, 2007). The transcript notes contained data written during and right after the interview as well as during transcription stage on participant's responses; personal notes contained information regarding the setting and researcher's reflections; analytical notes contained the ideas related to the analysis of research questions written right after or during the interviews (Ajjawi \& Higgs, 2007). Field notes assisted in my recall of data and context, thus helping me to separate my interpretations from the collected information.

Minimizing bias during the data collection process in qualitative research also differs from that in quantitative studies when it comes to interviewing the participants. While in quantitative studies researchers strive to prevent the variability of data resulting from the effects of the researcher, in qualitative studies researchers must be able to explain their influence on the data collection process (Maxwell, 2013). To minimize my influence on data collection during my interviews, I avoided asking leading questions (Maxwell, 2013). Minimizing bias during my interviews increased the quality of data I collected.

While qualitative and quantitative researchers have their differences in how they approach bias, phenomenologists have identified specific strategies they apply to minimizing bias in phenomenological studies. To counteract bias, some phenomenologists use epoche or bracketing to leave personal experiences with the phenomenon aside, to better focus on a phenomenon (Creswell, 2013; Heinonen, 2015). However, Van Manen (1990) teaches that it is 
impossible to eliminate researcher's bias from a qualitative research because researcher's experiences will still penetrate the study. Because assumptions and bias exist, simply ignoring them will not help to diminish their effect on the study (Van Manen, 1990). Thus, instead of simply acknowledging these experiences, a researcher needs to show how they affected the study.

\section{Methodology}

In this section, I explained participant selection logic, sampling strategy and size, inclusion criteria, recruitment strategies, interview protocol development, data collection procedures, data analysis plan, issues of trustworthiness, and ethical procedures.

\section{Participant Selection Logic}

A purposeful sampling technique was chosen for this study. When choosing participants for the phenomenological study, it is important that all subjects had experienced the phenomenon studied (Creswell, 2013). Therefore, the criterion purposeful sampling strategy was chosen (Creswell, 2013). Inclusion criteria for this study consisted of the following: current licensure, certification, and practicing in a role of an NP in the United States, having a collaborative agreement with physician(s), practicing for at least three months following the employer's orientation, and practicing for no longer than 5 years after graduation. Licensure and certification inclusion criteria were necessary because some NPs may have graduated but never worked in

their new role. This would have compromised data collection related to the role transition that occurs after graduation. Additionally, it was necessary to limit participants to those from the states that require the collaborative agreement with physicians to practice. This allowed the 
exploration of the preceptorship after graduation. The variability of the sample was also important to consider for the quality of received data (Patton, 2015). Thus, no limitations were applied to the different specialties or jobs where NPs chose to practice. Because role transition after graduation starts with practicing at a new NP job, it was important to include NPs at the different points of their role transition process, including those who have just started transition and those who have completed it. Those who had a doctoral degree in nursing were excluded from the study due to the differences between the masters and doctoral types of programs. Further, those NPs who have never practiced as NPs or those NPs who practiced for less than three months after graduation were also excluded because they have not experienced the second post-graduation stage of NP role transition. Those who practiced longer than five years after graduation were also excluded as they could have had more difficulties with recalling the information regarding their experiences with the role transition, which could have compromised the quality of data.

Snowball sampling technique was also used in addition to the criterion sampling method. Snowball sampling technique allowed me to locate the information-rich cases by asking my participants to share my research flyer with other potential participants (Creswell, 2013; Patton, 2015). Thus, criterion-based purposeful and snowball sampling techniques were used in this study.

\section{Sample Size}

The ideal guiding principle for the sample size decision was saturation of received information, which was achieved with data redundancy (Patton, 2015). Thus, while the minimum 
number of participants was predetermined; sample size may needed to be adjusted based on the length of the interviews the saturation principle (Patton, 2015). Creswell (2013) recommended approximately six to 10 participants for the phenomenological study. Mason (2010) examined 25 Ph.D. phenomenological studies and found that the sample size varied from seven to 89 with the mode of 20, mean of 25, and median of 20. Additionally, in her study of how faculty teaches NP students, Brykczynski (2012) interviewed 24 participants. However, in their hermeneutic phenomenological study of newly-qualified nurses' transition from student to staff nurse, and Kumaran \& Carney (2014) interviewed 10 participants. To generate quality data in my study, I used 12 participants guided by the data saturation principle.

\section{Instrumentation: Interview Protocol}

To collect data, at least ten semi-structured interviews that lasted up to one hour were conducted. Creswell (2013) recommended detailed and long interviews with 10 participants in the phenomenological studies. Because an interview protocol fosters comparing participants' responses to the interview questions and covering all needed topics in a limited amount of time, I used interview protocol in my data collection (see Appendix B for interview protocol; Patton, 2015). Field notes were also collected to capture participants' nonverbal reactions to questions, context specifics, researcher's reactions, and ideas (Ajjawi \& Higgs, 2007).

When collecting data about the lived experiences certain limitations exist. It is important to understand that data in the phenomenological study is collected retrospectively; thus, it is never exactly the same with the lived experiences of the participants (Van Manen, 1990). In this study, I collected data through interviews, which is consistent with the phenomenological 
approach (Van Manen, 1990). Van Manen (1990) warned to treat participants' written expressions of their experience with caution as they may include their explanations and interpretations. These interpretations may obscure the real lived experiences participants intended to share. Thus, Van Manen (1990) recommended for the researchers to instruct the participants on trying to avoid explanations, focusing on specific experiences and recalling as much as possible. During the interviews, Van Manen (1990) suggested to use silence and patience to allow interviewees to recall their experiences. However, if a researcher notices that interviewee starts generalizing, he recommended interjecting and asking the participant to give an example (Van Manen, 1990). Thus, I strived to obtain pre-reflective data free of participants' interpretations.

In constructing the interview protocol, different types of questions were used to elicit the information. First, the experience type of questions asking a person to describe their actions was included (Patton, 2015). Second, questions about participants' feelings were included to capture how they felt during the experience (Patton, 2015). Next, sensory type of questions asking participants to describe what they have seen or heard were utilized (Patton, 2015). Additionally, demographic questions included the information on gender, age, RN experience, number of months or years after graduation with NP degree, current employment status (including part time or full time or PRN), specialty, and the current number of MD collaborators (see Appendix A for demographic questions). Besides considering different types of questions in my interview protocol, I considered the time. More specifically, I asked my questions in past tense and present tense (Patton, 2015) to obtain data relevant to transition prior and after graduation. Further, I 
considered the sequence of my questions by asking easier questions about the experiences in the present time first, as people generally remember current information better; then I asked the same question in the past tense (Jacob \& Furgerson, 2012; Patton, 2015). Because Patton (2015) warned against asking many demographic questions at the beginning of the interview since they are usually boring and uncomfortable, I asked my participants to fill out the form with the demographic questions and e-mail it to me prior to the interview. Lastly, I avoided dichotomy questions to include open-ended questions in my interviews (Patton, 2015). Preparing good questions assisted me in gathering quality data.

\section{Procedures for Recruitment, Participation, and Data Collection}

To achieve a representative sample, the recruitment was approached adapting Arcury and Quandt (1999) recruiting site-based method. As suggested by Arcury and Quandt (1999), after determining the attributes of my desired sample, I evaluated the number of sites including the NP programs and NP organizations available to me. I was able to obtain the letter of cooperation from one of the nursing organizations in Mid-South area to have my research flyer distributed to the organization's members, when available.

In addition to the site-based recruiting procedure, I used the snowball recruitment method using my social and interpersonal connections to identify the potential study participants (Browne, 2005). Using snowball recruitment technique also assisted in increasing the variation in my sample since recruiting through the organization limited my participants to those NPs who are GMAAPN members only. My inclusion criteria were specified on my research flyer, which was distributed to the potential participants via e-mail. After the research flyer was distributed, 
potential participants were free to contact me. I determined the eligibility of participants and offered my participants the choices of time and place for the interviews. I obtained my consent via e-mail to ensure that my participants had sufficient amount of time to read the consent and ask questions prior to the interviews.

Congruent with the research design and paradigm, methods of data collection included semi-structured interviews. Participants were offered several options regarding the location of the interviews including participant's home, local library (if a participant was located in Memphis, TN), or Skype. Each participant was interviewed once. The duration of the interview was up to one hour. The interviews were audio recorded. Field notes were taken during and right after the interviews to capture the context, researcher's ideas, and nonverbal clues. At the end of each interview, I summarized participants' responses to ensure understanding and that no information was missed. I asked participants for permission to contact them if I had any followup questions. I also provided participants with my contact information in case they would like to contact me with any questions. In case I was not able to recruit enough participants to satisfy the requirements of my study, I had obtained a preliminary agreement of two Indiana universities that were ready to distribute my research flyer if I were to need additional participants.

\section{Data Analysis Plan}

All data in this study consisted of qualitative interviews. All data were transcribed manually by me using the MAXQDA qualitative data analysis software. The core of qualitative data analysis is coding; thus, to make sense of data, I analyzed it through coding. Coding is the process of assigning labels to portions of data (Miles, Huberman, \& Saldana, 2014). Coding 
should follow reading and "memoing" as it helps in "describing, classifying, and interpreting the data" (Creswell, 2013, p. 184). Coding was helpful in the early and ongoing analysis as well as in the data collection process (Miles et al., 2014). I performed coding to organize and reduce my data into meaningful fragments with assigned labels (Creswell, 2013). Creswell (2013) suggested developing a short list of five or six codes followed by expanding it to 25 to 30 categories with subsequent reduction of data to five or six themes. A theme is a broader category that contains several codes (Creswell, 2013). To maintain accuracy and consistency during the data analysis, I documented the codes' definitions. Defining codes helps the researcher to use them consistently throughout the study, as well as facilitate communication if multiple researchers are involved in a study (Miles et al., 2014). Providing the definitions for codes also strengthened the validity of data. Proper displaying of codes, using computer programs, assisted in data analysis. Displaying codes helped me to visualize the connections between the codes (Miles et al., 2014).

Additionally, I used jotting or memoing to document my reflections on my field notes. Jotting can strengthen coding (Miles et al., 2014). Displaying codes, connections, and my memos assisted in my analysis by improving my data recollection and thus, strengthening the validity of my analysis. Open coding and line by line phenomenological coding were used to analyze data through interpretive phenomenological analysis (Larkin \& Thompson, 2012).

When discrepant cases were encountered during data collection and analysis, I investigated and included them in my analysis. I first reviewed my transcripts to ensure that the discrepant finding was not a mistake in transcribing or interpreting (McPherson \& Thorn, 2006). Incorporating discrepant cases in the qualitative analysis may bring a unique point of view on the 
phenomenon that may enrich the results (McPherson \& Thorn, 2006; Miles et al., 2014). It is important to realize that discrepant cases or outliers can be represented by people, events, or experiences; examining outliers may be helpful in counteracting researcher's bias (Miles et al., 2014). Notably, sometimes outliers are represented by participants who are exceptionally bias (Miles et al., 2014). Thus, when dealing with discrepant cases, I first ensured they did not represent an error, then I determined the source of the outlier, then I reexamined my assumptions, and lastly, I analyzed the outlier, including it in my analysis.

Data analysis used in this study was consistent with the chosen hermeneutic phenomenological design guided by Van Manen's approach to data analysis. According to Van Manen's (1990), analysis of phenomenological data starts with making sure that the data collected was pre-reflective, thus, not interpretive. Next, Van Manen recommends using six strategies for data analysis along with four fundamental structures of one's life that can help describe any experience (1990). Lastly, the use of thematic analysis strategy is recommended (Van Manen, 1990). In the following section, I described my analysis plan in detail.

Data readiness and analysis. To perform a quality hermeneutic phenomenological analysis of my data, I first ensured that my data were conducive to the phenomenological analysis. To accomplish that, I reflected on my research question and the type of data received. To perform hermeneutic phenomenological analysis, the research question must be appropriate for the design and the collected data must be based on participants' experience (Van Manen, 1990). Questions that are abstract or those that enquire about participants' views or opinions will not lead to the insightful phenomenological results (Van Manen, 1990). Thus, prior the data 
collection I ensured that my research question and interview protocol were consistent with collecting pre-reflective data as opposed to data about my participants' opinions. After the data collection, I examined the data to ensure that it consisted of participants' descriptions of their experiences with their role transition while in a preceptorship. Phenomenological analysis must be presented in such a structure that displays rich description of the experience (Van Manen, 1990). After making sure my data fits the chosen hermeneutic phenomenological approach, I began with Van Manen's (1990) six components of the analysis.

Van Manen's methods. Van Manen (1990) offered a set of six procedures to assist with generating insight in doing hermeneutic phenomenology research. These procedures included the following, (a) "turning to the nature of the lived experience," (b) "investigating experience as we live it", (c) "reflecting on essence of the uncovered experiences," (d) providing rich description of the phenomenon through writing, (e) maintaining the orientation toward the phenomenon, and (f) considering different aspects of the research together with its whole (Van Manen, 1990, p.30).

In the first step of focusing on the phenomenon of interest, Van Manen (1990) underlined the importance of the research question being drawn from the researcher's specific interest or concern. Thus, the first step was to recognize that the chosen phenomenon of interest is connected to the researcher's orientation and to reexamine researcher's assumptions (Van Manen, 1990). For example, I looked at my phenomenon of interest from the perspective of an educator and practitioner. Being aware of my preexisting assumptions, I approached analysis with an open mind to capture the meaning of the phenomenon and to include prospective other than mine. 
The second step of "investigating experience as we live it rather than as we conceptualize it" was further explained as researcher's immersion or closeness with the experience (Van Manen, 1990, p.30). Therefore, the second step in the phenomenological study was to collect data using in-depth interviews (Van Manen, 1990). The data analysis was done on the descriptions received during the interviews; these descriptions affected the analysis. Both data collection and analysis cannot be separated (Van Manen, 1990). Only data relevant to the phenomenon of interest was included in the study. To avoid obtaining irrelevant data that may confuse the analysis, the researcher must always remember the research question to focus the interview during the data collection (Van Manen, 1990). Obtaining quality data that is not scant or overwhelmingly large was important for the quality of data analysis.

The third technique was "reflecting on the essential themes which characterize the phenomenon" (p.32). Thus, capturing the essence of the experiences with the phenomenon was commented on by exposing those things without which the phenomenon could not exist bringing the invisible elements of the phenomenon into the view through the data analysis (Van Manen, 1990). Thus, hermeneutic phenomenological reflection was used to approach the phenomenon and to capture the essences and meanings of the phenomenon (Van Manen, 1990). Creating and analyzing the themes through reflection was included in this stage of analysis.

Fourth, "describing the phenomenon through the art of writing and rewriting" was using language to show the phenomenon (Van Manen, 1990, p.30). In other words, writing about the phenomenon using reflection and analysis to bring the phenomenon in clear view in the researcher's write-up constituted this fourth stage of phenomenological analysis. Fifth, 
"maintaining a strong and oriented relation to the phenomenon," meant maintaining the orientation toward the phenomenon being free of "scientific" distractions by superficial, opinionated, or preconceived information while maintaining the focus on the essences of the studied experiences (Van Manen, 1990, p.31). Making sure that my analysis was data driven was the priority during this stage of analysis.

Lastly, "balancing the research context by considering parts and whole" must be employed during the data analysis (Van Manen, 1990 p. 31). Because it is easy to get lost in the small details when analyzing large amounts of data, a researcher is advised to continuously go back and forth from the smallest elements of data to the study's design, purpose, and question to ensure the alignment and logic of the study is maintained (Van Manen, 1990). The six techniques were not to be used in a stepwise approach as they were used in different order throughout the entire research process.

Fundamental structures. To further guide phenomenological analysis through reflection, Van Manen (1990) offered four "existential" themes including "lived space, lived body, lived time, and lived human relation" (p. 101). These themes are universal for all human living experiences, and any experience can be described by using these four categories (Van Manen, 1990). First, lived space is important because, just like writing at a particular desk at home may be more productive when working on a dissertation for many, space may have an important role in one's experience (Van Manen, 1990). Thus, space and various space-related elements such as distance and location may affect person's experience. Next, just like space, the physical body is always a part of one's experience (Van Manen, 1990). Considering physical body responses and 
reactions to experiences may be helpful in describing a person's experiences. Third, time, past, present, and future may determine one's experience (Van Manen, 1990). People may have different experiences depending on when they occurred. Additionally, what participants may share about their experiences may be influenced by the prevalence of their orientation toward past, present, or future time. Further, time may influence people's ability to recall their experiences. Lastly, the fourth fundamental element that accompanies every experience is one's relation to other people through interpersonal communication (Van Manen, 1990). A person's relationships with others may affect experiences. Thus, the four fundamental structures of each experience form the "lived world" of a person (Van Manen, 1990, p. 105). I used these four structures to describe my participants' experiences.

Thematic analysis. While thematic analysis may resemble coding process often used in qualitative research, it is worth mentioning it in a separate section since Van Manen tailored this process specifically to phenomenological coding process. To restate, the purpose of hermeneutic phenomenological research is to identify the meanings of the participants' experience with the phenomenon through examining the text produced through transcribing the interviews. To begin reflecting on the meaning of the text expressed by participants, one should evaluate the text as if it were composed of smaller units, or themes (Van Manen, 1990). The theme is defined as one of the commonly occurring elements of the text (Van Manen, 1990). It is important to understand that identifying themes in phenomenological research is different from performing the word frequency count and such (Van Manen, 1990). Thus, identifying themes helps to create a phenomenological description and make sense of data (Van Manen, 1990). In his approach to 
thematic analysis, Van Manen (1990) offered three strategies, including detailed, selective, and holistic reading approaches. In the detailed reading approach, each sentence is read, and its significance toward the phenomenon is examined (Van Manen, 1990). The selective reading method of identifying themes is when researcher examines text several times, highlighting those aspects that relate to the phenomenon the most (Van Manen, 1990). Lastly, the holistic reading approach is applied when researcher reads through the entire text and identifies one phrase that captures the essence of the phenomenon of interest (Van Manen, 1990). Conducting thematic analysis is to identify themes related to the meanings of participants' experiences (Van Manen, 1990). Thematic analysis leads to the circular analyzing between the smaller parts and whole parts of the phenomenon, sometimes referred to as hermeneutic circle (Patton, 2015). After identifying themes during the data analysis, Van Manen (1990) recommended identifying the essential themes through the "free imaginative variation" method when the researcher asks a question whether the phenomenon can still exist without the chosen theme (p.107). All three approaches were used in this study, aiming to capture the meanings of the studied phenomenon. While applying various techniques to this study analysis, I stayed true to the phenomenological approach. In phenomenological studies, insights are discovered rather than produced using methods only (van Manen, 2017a). Van Manen referred to phenomenological analysis as a phenomenological reflection that has an aim of capturing the meanings of the phenomenon (Creswell, 2013). Epoche and reduction are also related to phenomenological analysis. Epoche means suspending and eliminating bias to gain a fresh look at the phenomenon (Patton, 2015). The reduction is performed through bracketing of the existing knowledge and 
literature to reflect on the phenomenon of interest (Patton, 2015). Van Manen (1990) taught that epoche-reduction could be performed through staying open to data and explaining how researcher's bias and knowledge affect the study, as it is impossible to eliminate preexisting knowledge and biases.

\section{Issues of Trustworthiness}

\section{Credibility}

It was important for me to ensure that my data were truthful and of value. The credibility of qualitative data can be compared to internal validity in quantitative research (Miles et al., 2014). To establish my study's credibility, I ensured clear, detailed descriptions, prolonged engagement with participants, rich data, and triangulation (Miles et al., 2014). Additionally, I ensured the alignment in my study through providing consistency and transparency in data collection, analysis, and documentation. Prolong engagement with participants was achieved through conducting one-hour interviews. Longer interviews allowed establishing of trust between researcher and participant leading to a more comfortable discussion of the phenomenon by the participant. Triangulation in data collection was established through multiple data collection methods. Namely, to collect data, field notes and participants' descriptions were used. Additionally, multiple sources of data were used to capture different views of the phenomenon by including NPs who graduated from different NP schools and who practice in different settings, thus, establishing variability. It is important to note that because most of my data were collected retrospectively after participants have already experienced the phenomenon, my data depended on participants' recall. 


\section{Transferability}

Transferability or external validity measure in quantitative research is another quality indicator of qualitative research. The purpose of this study was to understand what was it like to transition to an NP role while in a preceptorship before and after graduation. Transferability to other professions' role transitions would be outside this study's aim. Readers will be allowed to make their own judgment regarding the applicability of this study's findings to their specific settings using the presented thick and clear description of my findings (Pringle, Drummond, McLafferty, \& Hendry, 2011). It is important to understand that the purpose of phenomenology is not to develop theories or generalize findings, but rather to uncover meanings from concrete experiences (Van Manen, 1990). Van Manen (1990) stated that anecdotes or the information gathered from the participants is a connecting link between the experience and the reflection as well as a link between "particular" and "universal" (Van Manen, 1990, p.120). Thus, while the data from single experiences provide information about the specifics of those experiences, it may also provide the connection to the understanding of the larger and more abstract phenomenon. Ultimately, it is up to the readers to decide whether they can transfer the finding of my study to their specific context.

\section{Dependability}

Data dependability in qualitative research can be compared to reliability measure of quality in quantitative studies (Miles et al., 2014). To establish dependability of my research, I ensured the quality of my data through triangulation and (Miles et al., 2014) through collecting field notes to capture context, audiotaping interviews to ensure accuracy, manual self- 
transcribing to ensure my deep understanding of data and using direct quotes from my participants. Additionally, my clear description of my researcher role and bias added to the dependability of my data. Further, using the same interview protocol for all study participants also was helpful in establishing that data were collected across comparable questions (Miles et al., 2014). I created a coding book to ensure the consistency of codes' descriptions and accuracy. I ensured that the data collected from my participants was pre-reflective as opposed to interpretive to ensure the accuracy of my results.

\section{Confirmability}

Confirmability of qualitative research can be compared with objectivity measure of quality in quantitative studies. To establish confirmability in my study, I retained my data for

reanalysis for five years before it is destroyed (Miles et al., 2014). I also kept a detailed record of my methods and procedures (Miles et al., 2014). Additionally, I ensured that my role in this research was described in detail including my assumptions, experiences related to the phenomenon, and biases (Miles et al., 2014).

\section{Ethical Procedures}

In addressing potential ethical issues in my dissertation study, I was guided by the three main principles of justice, beneficence, and respect for others. My first step in addressing ethical concerns prior to data collection included obtaining the approval from the institutional review board (IRB) for the process of data collection (IRB Approval Number 01-16-18-0522648). Ethical approval for this study was obtained from the Walden University. After disclosing the 
purpose of my study to the nursing organization's officials, I have received an agreement from the site; the site officials e-mailed my research flyer to its members.

At the beginning of my study, I contacted those participants who responded to my research invitation (Creswell, 2013). All study participants were provided with the written information regarding the study purpose and research process. This information was provided through the e-mail and prior to the interview. I sent an e-mail with the informed consent attached, asking participants to read it and emphasizing to participants that they do not have to sign it if they choose to not participate (Creswell, 2013). All study participants were notified of the voluntary nature of their participation as well as that they can withdraw from the study at any time without any undesirable consequences. I informed my participants that they did not have to print, sign, and scan the consent form back to me; all that was necessary, if they agreed to this study for them to respond "I consent" to my e-mail.

During the interview, I asked my participants if they had any questions regarding the consent or study. Each participant was given an opportunity to ask questions about the study. In my e-mail, along with the consent form and explanations about my study purpose, I informed my participants of the Amazon certificate of 10 dollars that they would receive after all interviews were completed through the e-mail as my compensation for their time. Creswell (2013) recommended using rewards to avoid having participants feeling "used.” Largent, Grady, Miller, $\&$ Wertheimer, (2012) reported that 94.2\% survey study participants (IRB members and research professionals) agreed that it is acceptable to compensate study participants for time; however, most IRB members study participants disagreed on offering money to compensate for the risk. In 
my study I offered my participants the small compensation for their time but not for their risk. Less than $20 \%$ of study participants noted concern about the "token" or small payments; IRB members and researchers were mostly concerned with the substantial payments and their effect on study participants' decision to participate. Latterman and Merz (2001) reviewed 109 research studies from ten journals and reported the mean monetary compensation used for interview studies of 24.40 dollars ranging from 10 to 100 dollars. Thus, my compensation of 10 dollars was minimal and appropriate.

Further, right prior the data collection, I again discussed my study purpose and how my data will be utilized (Creswell, 2013). Because no power relationships existed between the participants and me, no coercion took place during this study. I avoided bias and any power relationship by excluding any of my former students.

I discussed the confidentiality with my participants along with underlining the voluntary nature of their participation and ability to cancel at any time. Confidentiality was maintained by assigning participants with pseudonyms and preserving potentially revealing contextual details during results reporting (Creswell, 2013). I created a case book in which I coded my interviews and participants. This case book was locked at all times and I was the only person who had access to it. All transcripts had participants' case numbers or fictitious names and had no other identifiers. All data were stored in a password protected computer and a password protected external hard drive for five years. The data were accessed by myself and was shared with the dissertation committee only on as needed bases. 


\section{Summary}

In this chapter I have detailed the reasons for the chosen research methodology and methods. I have described my role as a researcher in my study along with justifying procedures for sampling, recruitment, data collection, and data analysis. Additionally, I have provided the description of how I maintained the trustworthiness of my study. Lastly, I have included my plan to protect data and my participants' confidentiality. In Chapter 4 I described the results of my study. 


\section{Chapter 4: Results}

\section{Introduction}

The purpose of this hermeneutic phenomenological study was to understand and describe the meaning of NPs' role transition experiences while in a preceptorship before and after graduation. The central phenomenon of interest was NPs' role transition while under the preceptor's guidance. It was important to find out how participants' transition was supported through preceptorship. The research question was as follows: What was the experience of transition like for an RN becoming an NP under the guidance of a preceptor during the academic program and after graduation? This question allowed participants to reveal role transition experiences while preparing for clinical practice, learning from preceptors, learning complex patient care elements, learning in clinical environments, becoming more autonomous, assuming new responsibilities, and finding a new role identity. This research question also helped to learn about participants' emotions and reactions to their role transition in the context of preceptorship (see Appendix E and D).

In this chapter, I will describe relevant participants' demographics, study sample, research setting, data collection, data analysis, and data trustworthiness as well as study results and summary.

\section{Research Setting}

The interviews were conducted via phone, Skype, and face-to-face. The research setting varied according to the interview and included a library, and the participants' or researcher's home. All participants were advised and confirmed being in a private and safe place during the 
interviews. At the time of study, there were no conditions that could influence participants nor were there experiences that may have influenced the interpretation of the study results.

\section{Demographics}

Study data were collected from 16 board-certified NPs who, at the time of the interview, were practicing in an NP role and had been practicing for at least 3 months but no longer than 5 years (see Table 1 and Appendix C). All participants were from states that required NPs to have a collaborating physician in order to practice. Those NPs who held a doctoral degree, attended a post-graduation residency program, or did not require collaborative practice agreement with the physician were excluded from the study. At the time of the interviews, most participants reported that they had been practicing in an NP role for less than 2 years. Most reported practicing in a specialty area, though all were certified to practice in primary care. Most participants had previously practiced in an $\mathrm{RN}$ role. Two participants reported to be direct entries into an NP program; they reported having no RN experience prior to, nor during, their NP program. Most participants had more than one NP job since the graduation and worked in an outpatient setting. Most reported having no formal preceptor assigned and minimal orientation provided during the NP job onboarding process; however, many reported having access to an informal preceptor at work. When in an NP program, most reported having the responsibility of finding and securing clinical sites and preceptors.

Table 1

Demographics

Demographic $\quad \% \quad n$


Graduated 2014-2017

Graduated prior 2014

In NP Role 4mo-2 y

In NP role $2 \mathrm{y}-4 \mathrm{y}$

Certified to provide $\mathrm{PC}$

$1^{\text {st }}$ job in PC

Worked in $\mathrm{PC}$ at the time of interview

Worked as an RN after graduated with NP

1-2 NP jobs since graduation

3-5 NP jobs since graduation

Works in NP role FT

Never worked as an RN prior graduated with NP

Works in out/patient setting now

Job orientation 0-30 days

Formal Preceptor Assigned at hiring

Able to access informal Preceptor

Has personal desk or office at $1^{\text {st }}$ job

Clinical Sites Were found by School
$81 \quad 13$

193

$\begin{array}{ll}75 & 12\end{array}$

$25 \quad 4$

$100 \quad 16$

$44 \quad 4$

$38 \quad 6$

193

$69 \quad 11$

315

$81 \quad 13$

122

$81 \quad 13$

$\begin{array}{ll}75 & 12\end{array}$

254

$63 \quad 5$

$\begin{array}{ll}75 & 12\end{array}$

315

Note. $\mathrm{PC}=$ primary care. $\mathrm{FT}=$ full time.

\section{Data Collection}

Prior to data collection, permission from the GMAAPN was received to e-mail research flyer to its 1016 members. The study flyer (see Appendix D) was then e-mailed by the organization's administrator twice over the period of three weeks to GRAAPN members. Additionally, after the permission to post the research flyer on Facebook to potentially reach 40,000 NPs was obtained, the study flyer was posted. Further, the e-mail informing about the 
research study along with the attached research flyer was sent to researcher's former and current colleagues asking to share research flyer and study information with others.

Data collection took place from January $17^{\text {th }}$ to March 6th of 2018. A total of 17 participants were interviewed, 16 of which were included in this study. One interview was excluded from the study when it was realized that the participant completed NP program outside of the United States. Though program completion location was not a part of the inclusion criteria, it was important for the study integrity that all participants graduated in the United States. It was then verified with other 16 participants that they all graduated from NP schools located in the United States. Several participants, who expressed the desire to participate in the study, were denied due to not meeting the study inclusion criteria or inability to devote one hour of time for the interview for proper data collection.

Those who wanted to participate in the study contacted me and the e-mail with the study inclusion and exclusion criteria was then sent asking the participant to review and confirm that they meet study criteria. Thereafter, an e-mail with the study consent and demographic form was sent to a participant asking to review the consent and let me know if any questions. If participants had no questions or concerns, they e-mailed me back the words "I consent" prior the interview as their agreement to participate. The demographic form was also returned by participants prior the interview day. It was reviewed it to ensure that participants met the study criteria.

One interview was face-to-face and was conducted in the private room of the local library. Three interviews were conducted via Skype with the participants being in their home and 
I was in my private residence. Twelve interviews were conducted using phone in private settings. Participants were instructed and agreed to choose private and safe locations for the interviews, which was verbally confirmed prior the interview start at the time of the phone call. The interview time ranged from 37 to 95 minutes. During the weeks of data collection one to four interviews were scheduled each week with one to two interviews a day. Each participant was interviewed once and was instructed to contact me if they would like to share more information or if any questions.

An interview protocol containing seven interview questions was used to interview all participants. Additional probe questions were asked as needed during the interviews. Filed notes were written after each interview to document important interview elements including interview setting, nonverbal reactions (in case of face-to-face and Skype interviews), and elements of conversations.

The data were recorded using two digital recorders to ensure back up in case of the technology malfunction. The audio recordings were then transferred to my password protected desktop computer and saved in the MAXQDA analysis program. All interviews were manually transcribed by me within the MAXQDA program. Participants' demographic data were entered into the MAXQDA. Each participant was assigned a unique number, which was used in the analysis as well as the report.

Besides excluding one interview from analysis due to the participant attended school outside the United States and the malfunction in both audio recorders for ten minutes during the first interview, there were no other variations or unusual circumstances in data collection 
methods from the proposed strategies. After the battery change, the recorders were again in use and the ten minutes of the first interview was handwritten and attached to the transcript. There were no complications experienced by participants during the interviews. All participants were offered to ask for a break at any time of the interview as needed.

\section{Data Analysis}

Data analysis used in this study was consistent with the chosen hermeneutic phenomenological design guided by Van Manen's approach to data analysis. In keeping with Van Manen's approach, I tried to collect pre-reflective data, which was as free of participants' interpretations as possible. My interview protocol questions were general enough to allow participants' descriptions. I then continued my analysis using Van Manen’s six analysis techniques.

\section{Van Manen's Six Analysis Procedures}

The first analysis step included "turning to the nature of the lived experience." During this step I put aside my background of an NP and NP educator and approached my data with an open mind to allow results to emerge (Van Manen, 1990, p.30). In doing so, I assumed a role of a listener in my interviews without offering any opinions. During the second step of "investigating experience as we live it," I clarified participants' questions as needed and offered probing questions when necessary to ensure that the data was relevant to my research question (Van Manen, 1990, p.30). During this step, I ensured that enough time was allowed to elicit data; I excluded those who were not able to allow sufficient time for an interview during the initial recruiting stages. The third technique was "reflecting on the essential themes which characterize 
the phenomenon" (Van Manen, 1990, p.32). During this stage of analysis, I looked for the essential elements of my participants' experience with role transition and preceptorship. To locate and document these elements, I used reading and coding techniques. My initial code count was 225 codes. Fourth, "describing the phenomenon through the art of writing and rewriting" was using language to show the phenomenon (Van Manen, 1990, p.30). During this stage of analysis, I analyzed my field notes and notes I made during the transcription process when rereading the transcripts. The fifth technique was about "maintaining a strong and oriented relation to the phenomenon;" it meant maintaining the orientation toward the phenomenon being free of "scientific" distractions by superficial, opinionated, or preconceived information while maintaining the focus on the essences of the studied experiences (Van Manen, 1990, p.31). I made sure that my themes and codes were derived from my data. My seven themes that emerged from data included transition preparation and learning, preceptorship during role transition and learning, transitioning to complex patients and learning, transitioning in clinical environment and learning, transitioning to a greater autonomy and new responsibilities, embracing the role and identity confusion, and transition reactions or rude awakenings. Lastly, "balancing the research context by considering parts and whole" must be employed during the data analysis (Van Manen, 1990 p. 31). During this stage, I made sure that the alignment among my data analysis, research question, and study purpose was maintained ensuring my analysis follows logic.

\section{Van Manen's Fundamental Structures}

To further guide phenomenological analysis through reflection, Van Manen (1990) offered four "existential" themes including "lived space, lived body, lived time, and lived human 
relation" (p. 101). These themes are universal for all human living experiences, and any experience can be described by using these four categories (Van Manen, 1990). I explored space by asking participants about their learning clinical environment; "P5: I was you know sad about I originally was going to be driving three to four hours one way, and literally, I had to fly across the country...for two weeks at a time, two different times to get my rotation done ..." I elicited the information about the living body by asking participants about their emotions and reactions to their experiences with transition and preceptorship; "P14: The way I transitioned, I felt like I was sink or swim, good luck; rude awakening because my experience has left me with more, I feel like more self-doubt, then I have ever felt before." I learned about the timing of my participants' experiences by asking them about their transition and preceptorship, both before the graduation,"P17: So in school, clinical experiences really depended upon how good of a preceptor location you got, honestly, like I felt like it was all left on us, so that was very difficult;" and after the graduation, "P17: So I really had to, I really had to learn on my own; P6: in my previous job I had to buy my own computer and I was told to practice on the weekend." I found out about my participants' relationships with others in relation to the phenomenon of interest by asking them about their communication with collaborating MDs and other professionals and staff in their clinical environments; "P14: Post-graduation I had collaborators on paper."

\section{Thematic Analysis}

Thematic analysis or identifying themes is similar to the coding process. Identifying themes helps to create a phenomenological description and make sense of data (Van Manen, 
1990). In his approach to thematic analysis, Van Manen (1990) offered three strategies, including detailed, selective, and holistic reading approaches. During the detailed reading approach, I read each sentence of my data transcripts and identified their significance toward the phenomenon (Van Manen, 1990); I looked to see what my data represents. I selected relevant portions of the text. (Van Manen, 1990). Lastly, during the holistic reading approach, I looked to see if my data makes logical sense (Van Manen, 1990). During this coding process, I identified multiple codes that emerged from the data.

Open coding was used while collecting data through interviews and during the manual transcription process. While the codes were created based on emergent data, each code was assigned a memo, which contained a short description of the included data. Seven categories that included data pertaining to the time before and after graduation were expanded to include over 225 codes, which emerged from data during transcription and line by line open phenomenological coding. Later, in the analysis process, data were reduced to seven themes and 34 subthemes. MAXQDA analysis program assisted in proper data display and easy retrieval of data.

I made sure that all the codes I identified were relevant to my research question. Thematic analysis leads to the circular analyzing between the smaller parts and whole parts of the phenomenon, sometimes referred to as hermeneutic circle (Patton, 2015). I deleted some codes that were not relevant and combined those that were into larger categories. Lastly, I used Van Manen's “free imaginative variation" approach to see if my themes were necessary to 
describe my phenomenon of interest (Van Manen, 1990). I was able to capture the meanings of my participants' experiences with the phenomenon of interest.

Two discrepant cases were encountered during data analysis. When these discrepancies were noted during the interviews, I clarified and confirmed these participants' former professional roles and their experience with an RN role. Participant 10 had never worked as an $\mathrm{RN}$; thus, despite him obtaining his RN degree prior to advancing to an NP role, he considered himself transitioning from his former role of a paramedic rather than $\mathrm{RN}$ role. Though this case was unusual, it brought my attention to the fact that there are programs in the United States that admit professionals other than RNs into the NP programs. This case was included in the analysis as it enriched the study results adding to the body of knowledge on the NP role transition.

The second discrepant case was Participant 14 who was also directly admitted into an NP program and transitioned into an NP role from the finance field. This participant took an RN and NP job at almost the same time after graduating with her NP. While unusual, this case helped me realize that there are people from fields other than health field who transition to an NP. This case was also included in my data analysis as it enriched my understanding of the transition phenomenon. The two discrepant cases were also helpful in counteracting my biases, as before conducting this study, I believed that only those who had practiced basic RN nursing were able to graduate with advanced nursing degree. 


\section{Evidence of Trustworthiness}

\section{Credibility}

It was important to ensure that my data were truthful and of value. The credibility of qualitative data can be compared to internal validity in quantitative research (Miles et al., 2014). To establish my study credibility, I ensured clear, detailed descriptions, prolonged engagement with participants, rich data, and triangulation (Miles et al., 2014). I ensured the alignment in my study through providing consistency and transparency in data collection, analysis, and documentation. Throughout the interviews, I validated participants' responses by restating and summarizing their descriptions to ensure my correct understanding of their statements. Prolong engagement with participants was achieved through conducting long interviews. Longer interviews allowed establishing of trust between researcher and participant leading to a more comfortable discussion of the phenomenon by the participant. To establish accuracy of data, I manually transcribed each audio recording and then used the MAXDQA analysis program features to display and easily retrieve data. Triangulation in data collection was established through multiple data collection methods. To collect data, field notes and participants' descriptions were used. Additionally, multiple sources of data were used to capture different views of the phenomenon by including NPs who graduated from different NP schools and who practice in different settings, thus, establishing variability. Two discrepant cases were identified, confirmed, analyzed, and included in my data analysis. These cases provided unexpected data that enriched the study results. It is important to note; however, that because most of my data 
were collected after participants have already experienced the phenomenon, my data depended on participants' recall.

\section{Transferability}

Transferability or an external validity measure in quantitative research is another quality indicator of qualitative research. The purpose of this hermeneutic phenomenological study was to understand and describe the meaning of NPs' role transition experiences while being in a preceptorship before and after graduation. Transferability to other professions' role transitions would be outside this study's aim. The participants included in this study resided and practiced in nine different states, outpatient and inpatient settings, as well as various NP specialties. Only those who were relatively recent graduates (years 2011-2017) were included in the study, which may be helpful when reflecting on the process of transition that took place more recently as opposed to decades ago. It is important to understand that the purpose of phenomenology is not to develop theories or generalize findings, but rather to uncover meanings from concrete experiences (Van Manen, 1990). Van Manen (1990) stated that anecdotes or the information gathered from the participants is a connecting link between the experience and the reflection as well as a link between "particular" and "universal" (Van Manen, 1990, p.120). Thus, while the data from single experiences provide information about the specifics of those experiences, it may also provide the connection to the understanding of the larger and more abstract phenomenon.

\section{Dependability}

Data dependability in qualitative research can be compared to reliability measure of quality in quantitative studies (Miles et al., 2014). To establish dependability of my research, I 
ensured the quality of my data through triangulation (Miles et al., 2014) and through collecting field notes to capture context, audiotaping interviews to ensure accuracy, manual selftranscribing to ensure my deep understanding of data, and using direct quotes from my participants. Clear description of my researcher role and bias added to the dependability of my data. Using the same interview protocol for all study participants also was helpful in establishing that data was collected across comparable questions (Miles et al., 2014). Additionally, using broad open-ended interview questions allowed for a reliable way of eliciting relevant information. I created a code book to ensure the consistency of codes' descriptions and accuracy. All memos and notes were directly linked to the themes, codes, and participants' transcripts within the MAXQDA system. I ensured that the data collected from my participants was prereflective as opposed to interpretive to ensure the accuracy of my results.

\section{Confirmability}

Confirmability of qualitative research can be compared with objectivity measures of quality in quantitative studies. To establish confirmability in my study, I ensured that my role in this research was described in detail including my assumptions, experiences related to the phenomenon, and biases (Miles et al., 2014). Describing and acknowledging my experience with NP programs and the NP role assisted me in staying objective when conducting this study. I used my background knowledge of the phenomenon to recognize the data that was relevant, unique, or discrepant. I also kept a detailed record of my methods and procedures (Miles et al., 2014); I shared my codes, code book, and analysis process with my dissertation chair and my committee member, qualitative methods expert to ensure and confirm that my inferences were correct. 


\section{Results}

The first four interview questions were related to the NP role transition element of role learning within the context of clinical environment. To explore how NPs learned their new role in the context of preceptorship, the four attributes of a successful preceptorship were employed (see Figure 1) to guide the interviews. These four attributes included NPs' experiences with readiness and preparedness to learn through experience, preceptors' teaching methods, learning complexities, clinical environments, and communication.

\section{Theme I: Transition Preparation and Learning}

The first interview question was related to the concept of baseline knowledge, exploring NPs' preparedness to learn in clinical environment. The two transition stages, pre and postgraduation from an NP program were explored.

Pre-graduation: Transition preparation and learning. Participants revealed four major themes related to preparedness to clinical experiences while in the program; including meet and greet orientation, EMR and charting, expectations in clinicals, and didactic-clinical overlap (see Table 2).

Meet and greet orientation. Participants reported that they had no orientation to their clinical preceptors and clinical sites prior the start of their scheduled clinical experiences except for occasional short introductions initiated by the Participants, "P2: I never had an orientation to a clinical site; P10: At each one of my sites I did go, introduced myself, met the preceptors, took brownies just to say hi." Instead, Participants mentioned that their clinical hours were used to provide for some basic orientation at the start of the rotations; the orientation lasted variable 
amount of days or weeks depending on the student's schedule and how quickly student and preceptor could adjust to each other. The lack of orientation to the clinical preceptors and clinical sites was reported to lead to the lack of preceptor and student trust and knowledge about each other, which transpired into the longer shadowing time to allow this trust and comfort level develop; however, participants reported that shadowing was not effective for their learning of NP skills.

EMR and charting. All participants reported some issues with the EMR access at their clinical sites. This access varied from having no access in all clinical rotations to being able to chart in most clinical rotations. Results showed that participants changed clinical rotations frequently and their EMR often changed with site change. Participants also reported that their inpatient clinical sites had EMR teaching documentation templates created for medical residents. Participants reported occasionally charting under their preceptor's log into the EMR. Additionally, it was reported that lack of EMR access affected participants' retrieval of patient related information necessary for making autonomous patient care decisions during the clinical learning experiences. Documenting while in the room with the patient approximated real life situations and was helpful for learning. Though EMR access was limited and inconsistent for all participants, they reported that the ability to document during their clinical rotations was imperative for their successful learning of their new NP skills. Because patient care documentation and coding is such a large portion of today's clinical practice reality, participants felt that its lack in their clinical training affected their role learning by not allowing their experience in clinical rotations approximate the reality of clinical practice. 
Expectations in clinicals. Participants reported that preceptors' expectations of students in clinicals varied significantly. Preceptors' schedules were rarely adjusted to account for having a student. Some preceptors expected students to 'jump in' taking care of patients right away, while others had students shadow them for a prolonged period. Some preceptors expected students follow evidence-based guidelines, while others preferred to use their established treatments instead. Participants reported lack of information regarding preceptors and sites' expectations prior to starting their clinical experiences and that schools would expect preceptors to teach students clinical knowledge, which was an unrealistic ad-on to an already busy preceptors' schedules.

Didactic-clinical overlap. When discussing their preparedness for clinical experiences at school, participants noted that having the alignment between the didactic work and clinical experiences was essential for their success in clinicals. More specifically, Participants stated that correlation of specific didactic courses with the corresponding clinical experiences was helpful. The lack of such alignment was reported when the NP student was faced with specific clinical situation that had not been reviewed in his or her course work. Having many didactic assignments during the time of scheduled clinical experiences seemed to be counterproductive as it limited the Participants' ability to focus on learning clinically related information. Participants mentioned that having visual instruction in a form of videos as well as having skill laboratory instructions appeared to be helpful in supporting their transition to a clinical environment. However, inconsistency with schools providing visual and lab instruction was noted among the participants' responses. Participants stated that limited clinical hours or having clinical 
experience scheduled once or twice a week, was inadequate because the NPs were unable to immerse into their new role and follow up on patients previously seen by them.

Table 2

Theme I: Pre-graduation: Transition Preparation and Learning

\begin{tabular}{lll}
\hline Code & Participant's Comment & Summary \\
\hline Meet and greet & P2: I never had an orientation to a clinical site... & Never had an orientation \\
orientation & & to clinical site
\end{tabular}

P10: Each one of my sites I did go, introduced myself, Show up the day of met the preceptors, took brownies just to say hi... clinical and start clinical aside from ... reading and studying the course experience material there was not a lot of additional training preparation before that, ...I would show up to my clinic and review the hours and see the patients...

P12: ...we just showed up and I think usually they had Show up the day of me kind of shadow the first couple of days and then clinical and shadow they would have me start seeing patients...

P14: you come in day one, you talk for 15 minutes, Show up the day of and go to see patient. clinical and have short introduction

P15: ...I stopped in to meet with them in advance and Short introduction prior just kind of just put a face with my name... clinical start

P16: ... every time I worked with somebody, my very Starting clinical with first day of clinical was my very first day working with observation their EMR, my very first day seeing patients. Normally 
like the first day in clinical when you start you kind of just shadow the other NP, would not really do much, you are just kind of watch what they are dong and how they are doing it and the system and what not.

EMR and charting P3: ... my 1 st and 2 nd and the 3rd I really did not get Respondent was able to to the EMR system so .... in my residency I did chart... chart in one of the four of her clinical rotations

P4: a lot of the clinicals I was not able to really use Mostly did not have any that EMR because I was a student and they just did not allow that access.

documentation experience in clinical rotations

P5: ...It was inconsistent if I had access to the EMR or No consistent EMR not, so sometimes I was able to look at things, access to learn patient sometimes not. related information to provide patient care

P7: I did some charting in their EMR, but all the Had an access to five EMRs are different and it just got a little bit different EMRs in five challenging and confusing when you are dealing with five different EMRs.

clinical sites but was not able to use them properly

P8: In one clinical I was able to go on chart in the room and that was really helpful because that what you Ability to document do in real life am and the one I was not able to open while in the room with chart in the room, so that kind of put me in more of a disadvantage. the patient approximates reality and helps learning

P10: out of my clinic sites, there was only one site that I could get on and use the EMR...

Was able to use EMR at one clinical site 
charting and the other one I did not chart.

P12: I know one place is paper still; ... so there I was able to make notes. I do not believe I charted when I worked like in ER or family practice...

P15: ... when I was with the hospitalist, he I allowed to go into their system and do some charting... in that system because of the teaching system they have an actual template for like residents or students and so I would go into their, and then my preceptor ... would just cosign my note

P16: ... couple of the places I was at did not want the students to chart so NP logged in herself and the student charted on the NP and then NP just checked note before she signed it...

Expectations in clinicals
P5: I finished my main courses for my NP and then was getting ready to go in the clinical. I did not feel prepared at all for what was about to happen in clinical. I don't feel like I understood what was going to be expected of me in clinical, especially since I had four different rotations and at least four different preceptors ranging from either an $\mathrm{MD}$ or $\mathrm{PA}$ or $\mathrm{DO}$ but never an NP so the expectations form each preceptor you know were definitely different.

P7: ...change in clinicals so new preceptors every couple months, so I definitely felt unsure of myself offered no charting experience

No experience with documentation except for one site (paper charting)

Hospital system had teaching documentation templates for medical students. Respondent had no experience with documenting except for the hospital site.

Respondent did not have own EMR log in.

Multiple preceptors, lack of clarity in what to expect in clinical rotations.
Frequent site changes and lack of consistency 
and in terms of what was expected from different preceptors in a clinical rotations, the confidence, I feel when I start to build my confidence up with my preceptors I transitioned to different clinic and it kind with every rotation...

P11: I feel that they expected our clinical preceptor to basically kind of teaching us everything we needed to know and that is not their job. Their job was to give us clinical experience not to teach us the basics.

P14: ...you basically follow them shadow them for that 1 day, but they really expect you to kind of jump in and start seeing patients...

Didactic-clinical overlap of start not back from ground zero but just ... basically

in preceptors'

expectations led to participant's decrease in confidence level

Unrealistic expectations of preceptors

Preceptors expected participant to jump in to seeing patients quickly

Didactic homework interferes with clinical experience to clinical the next day and you are not all the way there.

P5: ...part of the reason why I did not feel ready after Didactic homework graduation is because you are literally just trying to put interferes with clinical out the fire of that day just so you can continue on to experience the next one so it is not like you ever actually preparing and feeling confident and having the time to just take the information that you are learning...

P6: ...my school did a good job making sure that the Aligned didactic and course work or the classes aligned with whatever clinical courses clinical... 


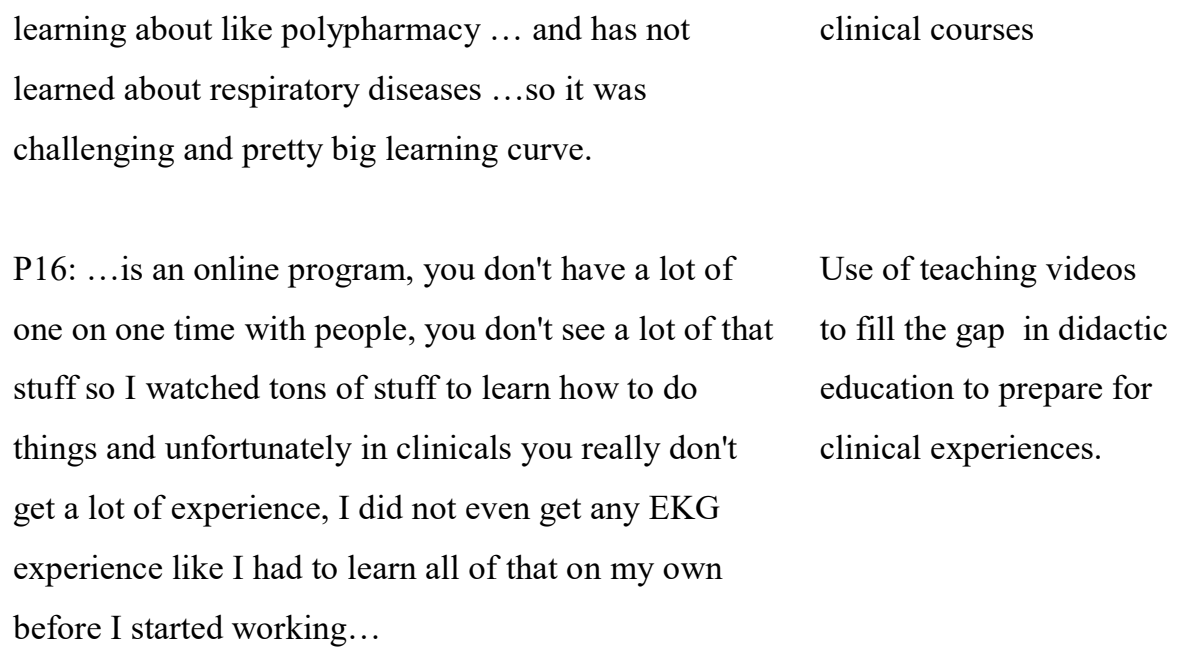

Note. Theme I / Pre-graduation / Meet and greet orientation /EMR and charting / Expectations in clinicals / Didacticclinical overlap codes

\section{Post-graduation: Transition preparation and learning}

When asked about their experience with readiness and preparation for transition to clinical practice, participants identified two major subthemes of preparedness and onboarding with several subcategories in each subtheme (see Table 3).

Preparedness. Participants revealed that they felt unprepared to independently function in their new role right after graduation. Superficial knowledge, lack of clinical experiences, as well as lack of procedural skill preparation, were underlined by the participants, "P10: I work urgent care (UC) and there was not a lot of procedural training in my program;" "P11: I feel like I did not really learn the stuff inside and out that I needed now in school.” Not feeling as being ready to fully function in a new role was reported to stem from the lack of exposure to various role requirements, including the process of diagnosing, clinical exposure various health condition 
opportunities, and different patient presentations. Participants consistently stated that their experiences in school clinical rotations often did not match the reality they encountered when entering the real-world practice.

Table 3

Theme I: Post-graduation: Transition Preparation and Learning

\begin{tabular}{lll}
\hline Theme/Code & Participant's Comment & Summary
\end{tabular}

\begin{tabular}{|c|c|c|}
\hline \multirow[t]{4}{*}{ Preparedness } & $\begin{array}{l}\text { P5: After graduation did I feel prepared, no I did not feel } \\
\text { prepared after I graduated either, I was actually scared } \\
\text { to death to go practice. }\end{array}$ & Feeling unprepared \\
\hline & $\begin{array}{l}\text { P10: I think I definitely had clinical preparation for } \\
\text { primary care and clinical management, but I work } \\
\text { urgent care (UC) and there was not a lot of procedural } \\
\text { training in my program. I don't feel necessarily prepared } \\
\text { to be an independent provider and the reason I chose the } \\
\text { NP route was to be independent. }\end{array}$ & $\begin{array}{l}\text { Lack of procedural } \\
\text { preparation; } \\
\text { unprepared to } \\
\text { independently } \\
\text { function in a role }\end{array}$ \\
\hline & $\begin{array}{l}\text { P11: I just felt like my particular program, it is a top- } \\
\text { rated program and I expected to come out of it and } \\
\text { knowing what I should know and I don't feel like it has } \\
\text { done that. I don't feel like when I came out really very } \\
\text { well prepared. But now in my new role I have added on } \\
\text { general pediatric and I feel completely unprepared for it, } \\
\text { because I feel like I did not really learn the stuff inside } \\
\text { and out that I needed now in school. I feel really } \\
\text { unprepared to be doing gen pediatrics, which that was } \\
\text { like what I trained to do. }\end{array}$ & $\begin{array}{l}\text { Unprepared to } \\
\text { function I a role due to } \\
\text { lack of solid } \\
\text { knowledge foundation }\end{array}$ \\
\hline & P12: Do I feel prepared, not really to be honest. & Unprepared \\
\hline
\end{tabular}


P16: I kind of felt I felt that school did not prepare me

Unprepared

all the way to be honest with you; like I got out and felt

like OMG I have got to do this now.

Note. Theme I / Post-graduation / Preparedness code.

Onboarding. The second code revealed by participants during the discussion about their preparedness for clinical practice was the onboarding code with the two major subcategories of onboarding structured elements and unstructured onboarding (see Table 4).

Participants identified several helpful elements of their training that took place during the onboarding period. These elements included EMR orientation and ramping up patient volume. Participants reported receiving short EMR training during the onboarding process, "P6: I had the time where... had a shadowing period with the director of informatics." Though having some EMR introduction was reported as helpful, many reported that it took longer to really learn EMR; thus, they were learning on the go; "P14: A lot of my EMR knowledge actually stems from working with different residents and attendings who have been there longer and showed me the ropes." Employers often expect NPs to see patients quickly and learning EMR to optimize documentation time becomes important.

Additionally, most participants reported that the number of patients per day was slowly advanced during the onboarding period to allow for learning to take place. Participants reported that a lot of self-learning took place during their transition to practice; self-educating and looking up the information related to patient care was reported time-consuming making NPs' appointments with their patients longer while adjusting; having only few patients on their 
schedule was helpful in being able to look up the necessary information or ask others for help; "P12: They only had me see like may be two patients a day on my first day, like just in case it took a long time to finish the note, or I had to look something up, or I had questions." However, several participants reported not having any other providers around who they could ask questions during this onboarding time. Some participants reported having to see full patient load right at the start of their first job. Additionally, several Participants noted that even though there were other providers practicing alongside with them, they often hesitated to approach them and only asked questions when unable to find an answer in any other way because of how busy other providers were; each of these experiences limited participants' learning. Lack of experience with the documentation in the EMR in NP school continued into having a minimal EMR instruction during the onboarding process. Having to spend additional time on learning how to navigate EMR in addition to learning how to manage patient care led to participants struggling with the time management. Though having additional time built into the NPs' schedules is a helpful strategy, lack of a structured onboarding process and a dedicated mentor minimized the effectiveness of this strategy.

Participants identified lack of structured orientation during their onboarding period. Two subthemes described by participants were hands off / winging it onboarding and learning through asking questions onboarding. Hands off/wining it onboarding was described as either no orientation; "P2: You kind of just get thrown in and figure it out," or passive shadowing of the provider while waiting for the credentialing process to be completed; "P11: My first job he gave me about like a month, where I shadowed another provider. He was very hands off, he never 
discussed clients unless I went to him specifically, we never had like a set time to sit down and discuss cases." Not having a structured onboarding process in place was reported as stressful experience of having to self-learn critical information. Though shadowing another provider was helpful in learning some of the information pertaining to patient population and the practice workflow, not being able to make autonomous patient care decisions during the shadowing period was reported as making the process of the orientation much less effective.

The second element within the unstructured onboarding subtheme revealed by Participants was learning through asking questions. Participants described that their orientation consisted of them asking questions of any of the available providers during the learning period; "P1: If I needed to ask questions to the pediatrician that was there or other NPs they were very helpful." However, because providers usually did not have a time carved out to help participants learn, asking questions was not always possible.

Table 4

Theme I: Post-graduation: Transition Preparation and Learning. Onboarding.

\begin{tabular}{lll}
\hline $\begin{array}{l}\text { Subtheme/Code } \\
\text { Onboarding }\end{array}$ & Participant's Comment & \\
\hline Structured & P1: And then the next day I was on my own with the & Ramp up Pt volume training \\
elements: Ramp & panel of seven patients I believe and then I got up to 14 & strategy \\
up patient volume & within the second week. & \\
code & &
\end{tabular}

P4: Like my first day I may be saw probably four Ramp up Pt volume training patients two in am and two in the afternoon and it kind of strategy stay that way for a while.

P6: I started having patients on my own schedule like Ramp up Pt volume training 
one an hour...

P8: Then I would go then I went to two patients an hour for about a month and then I went to three an hour, which is what I am at.

P10: For the first month for the first week I had one room and then two rooms, and then by the end of it I had three rooms just like the other providers.

P11: I recently started getting my own patient load and they are starting me out, three and three; I am going to move up to six in am and four in pm and then it will move up from there...

P12: They only had me see like may be two patients a day on my first day, like just in case it took a long time to finish the note or I had to look something up or I had questions.

P13: For the first month I had morning appointments only just so I had more time to try figure everything out.

Structured P14: Depending on the company I got one day Elements: EMR learning code orientation, the 1st job I got she had some guy come in just show me the EMR and how it worked.

P6: I had the time where... had a shadowing period with the, with have a director of informatics.

P14: A lot of my EMR knowledge actually stems from working with different residents and attendings who have been there longer and showed me the ropes. strategy

Ramp up Pt volume training

strategy

Ramp up Pt volume training strategy

Ramp up Pt volume training strategy

Ramp up Pt volume training strategy

Ramp up Pt volume training strategy

EMR orientation
Learning EMR from

residents and attending MDs 


\begin{abstract}
Unstructured:
hands off /

winging it

onboarding code

P1: I really did not get a precepting experience once I started my job. I literally got two days of orientation. The first day consisted of me sitting in a classroom, watching videos, just basic healthcare related videos. And then the second day I was with my supervising physician where he showed how to use the computer system. And then the next day I was on my own. Before the graduation that is what I expected, I expected to have.. I was thinking I was going to have all the support and help after I finish but that was not the case.

P2: You kind of just get thrown and figure it out; how much time the surgeon is going to take to orient anything formally, like realistically, none. I shadowed a neuro radiologist, and that stood out, they threw me with the bunch of med students and started asking questions about these scans like I did not know the answers to, so I did that and I went to other few things, that was about as formal as it got.
\end{abstract}

P3: ...there is nothing like that from the organization like a nurse like we go through the orientation how something no, no, no, it is not like that, it is just like if I have a question, I will ask him if I don't have a question I just go for it.

P4: Not like sit down and do case studies or nothing like that.

P6- I floated to two other sites and worked alongside different providers, it could have been MD, NP, or PA, so I kind of shadowed their work.
No orientation

Figure it out on your own

Nothing like orientation when graduated as an $\mathrm{RN}$

No learning through case studies discussions

Orienting though shadowing other providers 
P7: Unfortunately the NP has left the clinic like two months prior to me starting, so I did not get any orientation or any preparation in that manner, so no orientation in my transition to NP.

P8: It was like I was working one day and the lead doctor came up to me and said what are you thinking about going to two an hour tomorrow ha, I was like aaaaa, so it I feel it was not outlined and I was not mentally prepared.

P9: They just gave me an orientation for about a week to work with other NPs in the clinical to get an understanding of the patient population, the medications and prescribing, that was pretty much it, it was not really formal. You walk in, you put your bag down ... and you are on your own. I mean this is it... ... it is not any type of formal orientation...I have been looking for that and never got it.

P10: More or less it was training on the computer system, how to do ordering, I guess the mechanics of how to function within the clinic.

P11: My first job he gave me about like a month, where I shadowed another provider. He was not he was very hands off, he never discussed clients unless I went to him specifically, we never had like a set time to sit down and discuss cases.

P12: I think I got good medical knowledge from doing that but you won't get the time management, charting, you were not like I was not independently making the
No orientation

Unexpected ramp up

Housekeeping orientation

Housekeeping orientation

Hands off/shadowing orientation

Busy MDs preferred NP to have a shadowing/hands off orientation 
decision. A lot of my time was just with the physicians like watch them do procedures and also like in the office and it was really long, I want to say like 3 months. I was working with the physician a lot more shadowing I don't know they are just so time crushed that they don't have the time for you to go in first and then present the case, because they see so many patients a day, they are really busy, they kind of just preferred me to shadow I don't know.

P13: My employer did not have a very structured orientation for me. I learned a lot of things sort of passively because I was working with residents and a lot of questions were answered for them ha-ha, but my orientation was basically waiting for my credentialing.

P14: No, no, none of my jobs had orientations unfortunately. I $\mathrm{t}$ is something I always hoped for, especially considering that I am coming from a completely different field than health. He was not there my first day and he actually only was there once a week.

P15: That flow was that I was the first NP to be hired to work along with one of the surgeons (first job) and so they did not have an any orientation organized for me; it was not really structured, I mean that was frustrating but ... (next job) so I kind of just dragged along with the NP who has been there just for a year prior.

P16: I did not have like an orientation or any of that, I literally started the job and had to start working as an NP. Started function first day out I had to figure it out from the get go, I did not get any training I did not get any help with stuff.
Orientation by absorption

while waiting on

credentialing

No orientation

No orientation 


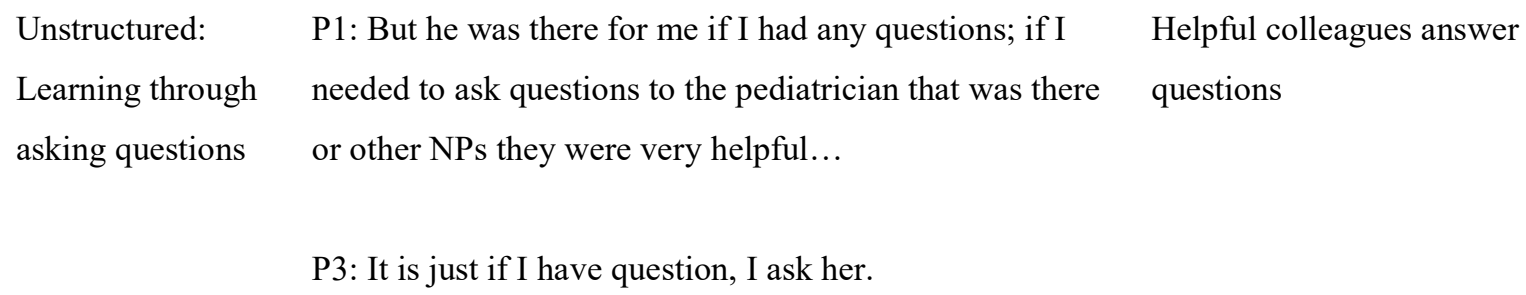

P14: Like for me coming in an ER, I am coming in never Learning through asking been in ER, so ask them a lot of questions, so whoever is attending MDs questions the attending, that day.

Note. Theme I / Post-graduation / onboarding subtheme.

\section{Theme II: Preceptorship During Role Transition and Learning}

Participants were asked about their experiences with learning from their preceptors while transitioning to their new NP role both during them attending NP program and after graduation.

Pre-graduation: Preceptorship during role transition and learning. When asked

about the transition time prior to graduation, participants identified one major category of preceptorship with three code elements related to their experiences with learning their new role from their preceptors. These included active learning, passive learning, and preceptor codes.

Active learning. Active learning code included participants' descriptions of their active learning experiences. The identified active methods of learning of the NP skills were combined in see one, do one, procedures, labs and coding, using resources, and student initiative code elements (see Table 5).

See one, do one. The overarching element related to semi-independent patient visits when student conducted the patient visit first, consulted the preceptor, received feedback with subsequent reassessment of the patient by the preceptor and a student, was reported as the most 
effective, hands-on way of learning when in the field; "P8: For the most part they would have me review the patient, go see the patient by myself, review my plan with them and then we would go back in together and then they would see the patient." Participants reported various degrees of conducting patients' visits semi-independently. Those participants who had more shadowing experiences noted that their experiential learning was much less effective. Active involvement in the patient care decision making process or learning by doing was reported as the most effective method of learning in the clinical environment. However, some preceptors were reported to be too busy to allow for such semi-independence in learning the role.

Procedures. Participants identified the lack of procedure training during their clinical experience in NP school, which included suturing, incision and drainage, X-ray reading, pelvic exams, and pap smear collection. Several participants reported having limited hours learning procedures in the laboratory; however, except for pelvic exams and pap smears, most never had an opportunity to practice procedures on the actual patients; "P16: So the only procedure I really got to do in clinicals and I got a ton of them is doing a pelvic exam. Otherwise most places that you are at are not going to let a student saw somebody up." Having no to limited exposure to performing procedures, lead to the lack of such skills when advancing to practice after graduation. Data showed that before the graduation, the lack of preceptors' time with the NP students translated into the lack of trust and comfort with the students, which in turn, led to decrease in hands on learning opportunities including procedures. After graduation, because of this gap in procedural training, participants reported being dependent on training provided on the job, self-learning, or other providers who were trained in performing procedures. 
Labs and Coding. Participants reported lack of experience with coding and with interpreting laboratory (lab) tests. More specifically, participants expressed lack of knowledge in managing abnormal lab results. It was particularly challenging for the participants to know what lab tests to order in response to receiving abnormal test results; "P11: Lot of my preceptors did not really utilize $\mathrm{CBC}$ in trying to figure out whether like something was bacterial or viral, when I got out into practice, I feel like I started to actually see that being used and I felt like, oh, that is something I should have learned in school like how to read a CBC..." Participants also noted that their infrequent clinical days played a role in that they were often not able to follow up on their previously seen patients and ordered tests. In addition to struggling with interpreting laboratory tests, participants noted having insufficient knowledge on how to perform coding; "P15: Some of the things that I did not feel as prepared for when I transitioned into that role was ... labs interpretations ... and then also billing and coding..." Ordering and interpreting tests along with correctly coding to receive appropriate reimbursement are essential role responsibilities that are necessary for the successful transition. Data showed that lack of consecutive clinical hours at a single site may be related to this gap in knowledge.

Using resources. Participants revealed that using guidelines and electronic resources such as medication databases (e.g., UptoDate), attending professional conferences, taking classes outside the NP program, as well as using patients' charts and EMR for learning, were effectively used to learn NP skills. Extensive use of resources was noted by participants in conjunction with their experiences of self-learning new NP skills, "P7: I also had Epocrates, UptoDate, and Medscape, I felt like I constantly found myself like looking up meds, I went to a couple 
conferences to kind of increase my knowledge base to prepare me for clinical." The use of resources was noted as an important part of role learning as participants stated that it was often up to them how much they got out of their learning experiences. The use of patients' charts to learn other providers' methods was found to be helpful, "P13: I can try to look up and understand like reading the progress notes and clinic notes of some of the other specialists or the family practice or whatever; I think I learned a lot that way by just reading the clinic notes of other people." Though EMR access was reported to be inconsistent and limited when in school clinical rotations, the importance of EMR and patient related information use continued into the learning experiences that took place after the gradation. When in practice, participants reported reliance on EMR to help them to sort out medication interactions and understand previously ordered patient medications. The skill of using appropriate resources and working with literature were reported helpful and continued being helpful after graduation.

Students' Initiative. Student initiative code element was revealed by participants when they were describing their way of obtaining information from the preceptor through asking questions, "P13: I asked a lot, I would ask questions of the preceptors" and requesting modifications for their clinical experience when inadequate,

P10: It was a family medicine site and the physician there agreed to trainme, wanted me to only shadow, and that is the extend. My school contacted her tried assist as far as expectations for my program, but at the end of the day we ended up having to cancel that particular rotation. 
However, participants reported that they often did not recognize that they experienced an issue of getting an inadequate clinical experience right away, which delayed the modifications. Taking an active part in their learning process was reported necessary in order to advance in their clinical skills, though not always possible due to the limitations of preceptors' time and availability.

Table 5

Theme II: Pre-Graduation: Preceptorship During Role Transition and Learning. Preceptorship/Active Learning

$\begin{array}{lll}\text { Subtheme/Code } & \text { Participant's Comment } & \text { Summary } \\ \text { Preceptorship } & & \end{array}$

/Active learning

See one do one

P1: Later she would send me to the room by myself like me

Learning by doing do everything and report it to him in a soap note format...

She basically let me watch her a few times and then she watched me, and then she would send me in and expect me and come out and have a diagnosis and treatment plan...

P2: ...preceptors stepped back but there were often Doing but with strong physically in the room, so I would like independently do supervision things... semi-independent I would say, like with very strong supervision.

P3: Most of my experiences I don't know it is they all let Learning by spending me go in the room first... she really liked me to spent time time with the patient with every single patient...

P5: I would go see the patients first.... before we would go Doing with preceptor's to see the patient together I would say this is what the explanations patient is here for, so I would say what my concerns were and so she would spend time saying yes I would agree 
these are the labs...

P8: For the most part they would have me review the patient, go see the patient by myself, review my plan with them and then we would go back in together and then they would see the patient.

P11: My preceptors at school I mean it was mostly learning by doing so like I guess.

P12: ...I would watch them do procedure first, like suturing, draining an abscess, or something. And then the second or third time after I saw, they let me do it with them watching, and then after they watched me do it a few times, they let me do it on my own, they may check it afterwards...

P13:...I would look them up and go and see them in the clinic first, and then once I saw them I would go and like check back with my preceptor and the preceptor would come back and we would see them again together and preceptor would finish the visit...

P14: ...so you would see the patient then you come out, give them report, present the patient, sometimes they will come in and see the patient with you depending on obviously it was patient dependent...

P16: So some of them would have me go in by myself and see the patient first, and then come out and present the patient to them and then go in I would go in with them and then see how they would run the visit afterwards, other preceptors would go in with me and we would do the visit
Doing first, then see

patient together

Learning by doing

Watch first, then do one with preceptor, then do one independently with preceptor's follow up

Doing first, then see patient together

Doing first, then see patient together sometimes

Different preceptors different styles 
together, like they would let me kind of run the show but would ask additional questions...

Procedures

P2: So I would, may be, watch them twice and then, start trying to help, do little things, but of course fully

Slowly starting learning supervised...

P11: We had a lab on suturing but again I have not done it on actual patient. I have not done I and Ds at all.

Have not done

procedures

P12: ... however like procedure wise like when I did my

Has not done any pap $\mathrm{OB}$, I have never done I had never done a pap smear, I kind smears. of felt really unprepared for that ... but we never had like live model or anything I know some places do stuff like that. I did a lot of my clinicals in ER, UC area, so a lot of times I would watch them do procedure first, like suturing, draining an abscess or something. And then the second or third time after I saw they let me do it with them watching...

P14: No no, unfortunately all of my rotations I ye, no hands Have not done on, I think that is where my training I felt lacking because I procedures ended up in ED having to suture, and did not even have a suture class which I was really surprised about going through the program, ye, so there was no hands on like in terms of procedures...

P15: No, no I don't think I did do any of those with my Have not done any preceptors, I mean, the only thing of course it is women's procedures except for health with doing pap smears and that kind of things but as pap smears far as I and Ds...

P16: So the only procedure I really got to do in clinicals Have not done any 
and I got a ton of them is doing a pelvic exam. Otherwise most places that you are at are not going to let a student saw somebody up.

Labs and coding

Using resources procedures except for pelvic exams

Never learned CBC interpretation in school viral, so when I got out into practice, I feel like I started to actually see that being used and I felt like o that is something I should have learned in school like how to read a CBC...

P13: I would say in my cardiology rotation I did some of that cause I had some basic instruction... That was mostly just like basic metabolic panel though. There was not anything more complicated than that... in the clinical did I do a whole lot of it No, I don't think.

P15: Some of the things that I did not feel as prepared for when I transitioned into that role was just one thing is like labs interpretations I wish that was a little heavier in our curriculum and then also billing and coding...

P3... we always looked up UptoDate, the new guidelines...

P7...I did a lot of reading obviously we were studying in school and reading the books... I also had Epocrates, UptoDate, and Medscape, I felt like I constantly found myself like looking up meds, I went to a couple conferences to kind of increase my knowledge base to prepare me for clinical.
Some lab interpretation in didactic course, but none in clinical rotations
Not prepared to interpret labs and to code
Using UptoDate

Database

Using UptoDate.

Epocrates, Medscape,

texts, and conferences

P8:...I tried to... that additional resources to bring with me, Using charts from 
so for example like pediatrics I got like a couple references

that I can bring with me, like the ... handbook and some

charts got from Amazon...

P9...NP review book that I reviewed and... with me to

clinicals and I could look up information when I had

questions...

P13...I can try to look up and understand like reading the progress notes and clinic notes of some of the other specialists or the family practice or whatever; I think I learned a lot that way by just reading the clinic notes of other people.

Students' initiative P1: If I had a question, we would talk about it after each patient. None of us did a debriefings, may be my first preceptor.

P7...I asked a lot of questions during clinical of my preceptor...

P9: I learned from them discussing different cases, asking my questions if I had questions, pretty much it.

P10...I had practically zero women's health. So I just asked for an elective and they found that one for me.
Amazon

Using NP review book

Reviewing others' notes

in patients' charts

Discussed cases only if student initiated

Learning through asking questions

Learning through asking questions

Participant requested

clinical experience

(women's health) since

it was completely

missing form his

rotations

Participant reported

"shadow only" preceptor

to his school 
expectations for my program, but at the end of the day we ended up having to cancel that particular rotation

P12:...mostly just making sure that you asked whoever was who you were following for your clinical, so you were not afraid to ask questions or tell them you have never done something and ask them to show them to you, it was kind of like I kind of felt a little bit on my own, you know it is up to me to learn this and learn how to do it.

P13...I asked a lot I would ask questions of the preceptors...
It was up to participant

to ask for those experiences that were missing in her clinicals

Note. Theme II. Pre-graduation: Preceptorship Category. Active Learning Code.

Passive learning. In addition to the active learning element of preceptorship, a passive learning code element was also identified from data. Passive learning code element includes See all, do none and limited patient care opportunities components (see Table 6). Participants reported having at least one clinical rotation (often women's health rotation) in which they were allowed to shadow only, "P11: It is hard to learn when you are shadowing in general because you just don't. You are not doing it." Additionally, shadowing was also reported to be used in the beginning of all students' rotations,

P15: It be like a couple of weeks into the clinical setting before that comfort level would kind of develop to where they would realize that they would be comfortable, and their comfort level with me to trust that my assessment were appropriate... The length of the shadowing period varied (e.g., from one to four clinical days per rotation; students reported having approximately four to five rotations per program) and 
depended on students' schedules and preceptor's comfort level with the student. Participants reported such hands-off observation experience as being ineffective in helping them to learn NP skills during their transition, but necessary to build their preceptors' comfort level with them. Participants reported lack of specific patient care opportunities depending on the setting. More specifically, some participants reported that they have never performed a well child exam, rectal, or pelvic exams,

P16: I never got any experience in well child visits at all, so like I saw a lots of sick kids, so once I started practicing on my own, I felt comfortable about that, but I have never done a well child visit, so I had no idea how to go, like the things I would look for or what not...

Though observation time was not as effective in NPs' learning their new skills, it was necessary for both, students and preceptors to build trust, develop comfort level, and learn about each other.

Table 6

Theme II: Pre-graduation: Preceptorship during role transition and learning. Preceptorship / Passive Learning

\begin{tabular}{lll}
\hline $\begin{array}{l}\text { Subtheme/Code } \\
\text { Preceptorship/ }\end{array}$ & Participant's Comment & Summary \\
Passive Learning & & \\
\hline $\begin{array}{l}\text { See all, do none and } \\
\text { limited patient care }\end{array}$ & P1:..the only rotation that I did not have any type of & No hands on in \\
opportunities & hands-on experience or really was kind of just an observer, & Women's Health \\
& get to measure fundus, I never got to feel how dilated she & rotation \\
& was or anything like that, I basically just watched... &
\end{tabular}


P3...do the examination, except the pelvic exam, ye except No pelvic exams the pelvic exam, so basically I do all the other examinations ... I did not do the pelvic exam...

P3: my pediatric rotation we do have a couple of instances the kids come in needed to be sutured you know, so I just observed, ye, that was my first rotation...so in that rotation I really did not get much independent experience... my first clinical started with pediatric, so I did not get to see patients as independent as I expected, so I keep trying to prove to my preceptor, I can see this patient by myself...

P4:...probably with every clinical that I did probably the first week or two weeks I was in clinical, just kind of shadow...

P6... I was still my first semester I did a little bit more shadowing, but then I had MD preceptor so I did not get to really embrace or understand my NP role because I did not have NP preceptor, so that was a little bit different...

P7: All my preceptors were so different, one of my preceptors she would see the patient and you know kind of have me shadow her and follow her and then just kind of watch her and learn that way... she has her own pediatric practice and has been practicing for like 40 years or something so I think she was tired so she just wanted me to shadow her...

P8:...sometimes the physician preceptors were more like observation a little bit, and they just kind of were not always I feel like it was not as interactive of an experience...
Little to no hands on in pediatric rotation. Observed procedures only

First week or twoobservation only Was not able to observe role modeling since the preceptor was MD

Only shadowing in one rotation

MD preceptors were less interactive with the participants and allowed for less 
independency

P9... started out shadowing them, and eventually work up to seeing patients with them... mostly shadowing, the women's health I could not go in by myself because the...

$\mathrm{P} 10 \ldots$ it was a family medicine site and the physician there agreed to precept me wanted me to only shadow, and that is the extend. And the rationale was that she had a private clinic and it is not a teaching environment and her patients' expectation was seeing her...

P11:...she like did not really like let me see things a whole lot, it took a really long time before she kind like let me go in and do my own assessment present to her, so I kind of felt I shadowed a lot longer than maybe I should have and so I did not feel like I learned much there...shadowing it is hard to learn when you shadowing in general because you just don't. You are not doing it.

$\mathrm{P} 12 \ldots$ it was a really, a lot of even just shadowing which I don't think it is quite as beneficial...

P15: It be like a couple of weeks into the clinical setting before that comfort level would kind of develop to where they would realize that they would be comfortable, and their comfort level with me to trust that my assessment were appropriate.... unfortunately it was mostly observation, and not once during clinical did I do a prostate exam...

P16: Normally like the first day in clinical when you start you kind of just shadow the other NP, would not really do
Only shadowed in

Women's health

rotation

Only shadowing in Family Practice rotation

Shadowed much longer than should have. Can't learn.

Shadowing is not as beneficial

Never had performed a prostate exam during clinicals. The length of shadowing depended on preceptor's comfort with the participant

Shadow in the beginning of each 
much, you are just kind of watch what they are dong and clinical how they are doing it and the system and what not.

P16:...I never got any experience in well child visits at all, so like I saw a lots of sick kids so once I started practicing on my own I felt comfortable about that but I have never done a well child visit so I had no idea how to go, like the things I would look for or what not...
Never had performed a well child exam during the clinicals

Note. Theme II: Pre-graduation: Preceptorship. Passive Learning.

Preceptor. The third code identified within the preceptorship category was preceptor code. Participants identified their preceptors' characteristics and behaviors that played a role in their clinical experiences at school. Among these were preceptors' workload, feedback, differences between NP and MD preceptors, role modeling, teaching methods, as well as approachable and encouraging preceptor (see Table 7).

Preceptors' workload. Participants noted that their preceptors had more time to teach them when preceptors' patient volume was either lower or adjusted to account for having a student, which was rare, "P4: His day was never swamp with the whole lot of patients, so he really had the time to actually teach me..." In most situations, participants described their preceptors as being very busy due to high patient volume or having medical students and not having sufficient time to teach them, "P16: She would see at least 40 patients a day and so she was too busy to explain anything at all. So I did not get a lot of teaching from her.” However, having high patient volume was also described as necessary for students to have enough patient care opportunities. Participants reported that the downside of their preceptor being busy was that in some instances students were used as an extra set of hands or their shadowing time was 
increased; "P3: She is pretty busy, she sees 25 patients at least, 25 in 8 -hour period. So it is just...bum, bum, bum... I just follow her, yeah, I just follow her.” Furthermore, some reported feeling being a burden to an already busy preceptor, "P12: You feel like you are tagging along ha-ha, like their job would be easier if you were not there." Overall, not having a carved-out time for a student decreased the quality and effectiveness of participants' clinical experiences at school clinical rotations. To provide NP students with quality clinical education, preceptors should have a dedicated time to do so; however, data showed otherwise.

Feedback. Participants reported that the amount of direct ongoing student feedback provided by their preceptors was minimal. Most feedback was limited to filing paperwork by the preceptors during midterm and at the end of the rotation, which was then sent to school, " P14: They get something back from...university and ...university gives then back something and I am not sure what, it was a very structured process, in terms of feedback...." It was unusual for participants to report having debriefing sessions at the end of the clinical day with their preceptors to discuss patients; however, depending on the time available and the specific cases, participants were able to ask occasional questions after seeing each patient on an as needed basis, "P8: We kind of debriefed after each patient at one time and then sometimes usually at the end of the day they would say, good job today, make sure you read about this condition..." The ongoing feedback was reported to be inconsistent and situation dependent. Some participants reported some of their preceptors gave them specific home assignments on a regular basis. Outside of the practices' normal workflow process, which included interactions with the 
preceptor and assignments given by some preceptors, no other evaluation procedures of NP skills by the preceptors were reported by the participants.

NP or MD preceptors. Participants reported that their experiences with MD preceptors were less interactive and included more observation time, whereas NP preceptors seemed to trust NP students more with providing patient care semi-independently,

P7: My NP preceptors were great, they basically wanted me to kind of run the show and to be assessing and diagnosing, creating a plan, whereas the MD kind of had me a little bit more like shadowing and kind of following them.

Participants also reported that MDs' expectations for NP students were less clear than those of NP preceptors, "P8: NP preceptors were more like understanding of my process and what I would be expected to know." Having NP preceptors was reported to be helpful because NP preceptors were most familiar with NP students' needs and expectations.

Role modeling. Participants noted the importance of their preceptor's professional role in their learning of the NP role. While learning their new NP role, participants reported mimicking their preceptors' attitudes and behaviors, "P13: I just sort of followed around and just did what preceptor did ... however they try to act toward the stuff, I was trying to act toward the staff, trying to just mimic whatever they were doing." Participants reported having various preceptors during their rotations including, MDs, NPs, PAs, and Dos, "P5: I had four different rotations and at least four different preceptors ranging from either MD or PA or DO but never an NP, so the expectations form each preceptor you know were definitely different." Participants reported that they were able to look up to NP preceptors as role models because NP preceptors were more 
familiar with the NP role. Having a role model was important for effective learning of the new role.

Teaching methods. Explaining was identified by the participants as one of the methods preceptors used during their preceptorship, "P5: She was more focused on making sure that I understood the thought process as to what I was finding..." Participants stated that explaining helped them understand the thought process and the rationale for the preceptor's actions. Additionally, giving students specific assignments was also reported as one of the strategies preceptors used,

P1: They had me go home and read, one of my preceptors asked me questions every time I came in and if I did not know the answer she would not tell me, she would have me go back and look it up.

Preceptors' directing students to specific guidelines and resources was identified as one of the teaching methods that was used by preceptors,

P13: Three of them, like if I have questions, would usually direct me to like look things up...so I feel like they helped me to identify where my reading resources are and where I should be looking for things, which in clinical practice is very helpful to me... Participants reported continuing using this skill of searching the information and working with literature after graduation. Overall, preceptors' explanations, specific assignments, and guidance to appropriate resources were identified as helpful in NPs' learning their clinical skills. Approachable and encouraging preceptors. Participants described preceptors' approachability and encouragement as being positive attributes of their experiences allowing 
them to learn the information more effectively in an unfamiliar clinical environment. More specifically, those preceptors who were approachable and encouraging fostered NPs' selfconfidence, “P7: So she really trusted me and allowed me to just do everything, which upped my confidence and allowed me to feel confident with doing my role as a NP on my own...," autonomy, "P11: She was like also very approachable and really laid back... and she let me do a lot and encouraged me," and the desire to ask questions, "P14: If you had question, you can certainly approach them and I was fortunate, all of my PNP preceptors were very approachable." Thus, preceptors' positive personal characteristics facilitated participants' learning process.

Table 7

Theme II: Pre-graduation: Preceptorship during role transition and learning. Preceptorship. Preceptor.

\begin{tabular}{|c|c|c|}
\hline $\begin{array}{l}\text { Subtheme/Code } \\
\text { Preceptorship / } \\
\text { Preceptor }\end{array}$ & Participant's Comment & Summary \\
\hline \multirow[t]{3}{*}{$\begin{array}{l}\text { Preceptor } \\
\text { workload }\end{array}$} & $\begin{array}{l}\text { P1... he took a lot of NP students at one time... unless we } \\
\text { were behind he would expect us to go and do vital signs so } \\
\text { when he goes in the room the patient was ready... }\end{array}$ & $\begin{array}{l}\text { Busy preceptor has } \\
\text { multiple students }\end{array}$ \\
\hline & $\begin{array}{l}\text { P12:...you know they are busy, especially like family } \\
\text { practice, you know may be } 40 \text { patients a day, so I don't know } \\
\text { if they had time to sit down and explain a lot of stuff to me but } \\
\text { they would answer like little question here and there or let me } \\
\text { use their UptoDate on the computer... }\end{array}$ & $\begin{array}{l}\text { Busy preceptor has } \\
\text { high patient load }\end{array}$ \\
\hline & $\begin{array}{l}\text { P3: Well, she is pretty busy, she sees } 25 \text { patients at least, } 25 \text { in } \\
8 \text { hour period. So it is just...bum, bum, bum... I just follow } \\
\text { her, ye, I just follow her... } \\
\text { P4...his day was never swamp with the whole lot of patients }\end{array}$ & $\begin{array}{l}\text { Busy preceptor- } \\
\text { participants ends up } \\
\text { having more } \\
\text { observational type }\end{array}$ \\
\hline
\end{tabular}


so he really had the time to actually teach me...

P5: One of the preceptors it was for my women's health and $\mathrm{OB}$ rotation she actually told the office manager to decrease the number of patients that she was seeing just so she could have more time to talk me through things and spend more time with me seeing patients...

P5... I think for the most part they were pretty happy to have students there other than obviously sometimes having a students and explaining things to a student can slow things down and it is not like they are nonprofit, they are there to make money so if you slow things down you know and as a preceptor my school did not compensate these preceptors at all, so they are doing it literally out of goodness of their heart...

P7...everything pretty much stayed the same, we were just in addition to the team, so they did not change added or dropped patient when there was a student.

P12: You feel like you are tagging along ha-ha, like their job would be easier if you were not there.

P15: If she felt like she had if she had something she had to get to at the end of the day, appointment or one of her children's ballgames or something and she did not want to carry over, she would like hassle through the schedule and sometimes would say o, I will go with you on this one or I will just to kind of hurry the schedule along.

P16...so I have one location where I was at, she would see at least 40 patients a day and so she was too busy to explain experience

Preceptor adjusted

her patient volume to

be able to teach

Having a participant

would slow down the preceptor

No adjustment for having a student

Feels like a burden to a preceptor

Preceptor is in a hurry to leave

Too busy to explain 
anything at all. So I did not get a lot of teaching from her, I

was used more like an extra set of hands...

\begin{tabular}{|c|c|}
\hline \multirow[t]{3}{*}{ Feedback } & $\begin{array}{l}\text { P7:... we would do like a midterm review and then a final } \\
\text { evaluation but I would always asked my preceptors if there } \\
\text { was they wanted me to do or they wanted me to change... }\end{array}$ \\
\hline & $\begin{array}{l}\text { P8: We Kind of debriefed after each patient at one time and } \\
\text { then sometimes usually at the end of the day they would say, } \\
\text { good job today, make sure you read about this condition } \\
\text { because you were not really sure what to do about it, you } \\
\text { know that kind of thing... }\end{array}$ \\
\hline & $\begin{array}{l}\text { P11: They had a form that they filled out am at the end of } \\
\text { preceptorship for that is how we were doing, so just filled out } \\
\text { that paper and then I think it was a piece of paper so I am } \\
\text { pretty sure I took it back to school and they put it in my file or } \\
\text { whatever }\end{array}$ \\
\hline
\end{tabular}

Feedback per

student's request

Debriefing after each

patient

Feedback on paper

P11: ... it was just like the day is over and we are ready to go

No feedback

home.

P13: Depending on how time allowed.

Feedback if time

allowed

P14: They get something back form...university and

Feedback on paper

...university gives then back something and I am not sure

what, it was a very structured process, in terms of feedback...

NP or MD $\quad$ P7:...my NP preceptors were great, they basically wanted me

More independence

preceptors

to kind of run the show and to be assessing and diagnosing,

and trust with NP

creating a plan whereas the MD kind of had me a little bit

preceptors

more like shadowing and kind of following them. 
P7:...my NP I would say more a little bit better about challenging me and giving assignments to focus on when leaving clinic, so they would ask me you know study contraception options or like JNC guidelines, whereas the doctors with my pediatrician preceptor I had she would just tell me everything was fine, everything was good

P8:...NP preceptors were more like understanding of my process and what I would be expected to know and I feel like sometimes the physician preceptors were more like observation a little bit, am and they just kind of were not always I feel like it was not as interactive of an experience...

P9...I learned most from the NPs than the MDs, in my clinical internship.

Role Modeling

P1: I feel like from that preceptor I learned how to really be a companionate for the patient working with the soldiers, she just was really nice...

P5: I had 4 different rotations and at least 4 different preceptors ranging from either from an MD or PA or DO but never an NP so the expectations form each preceptor you know were definitely different.

P13: I think that my preceptors I had, the NP preceptors I had were all very good, they kind of gave you the role model, showed you what like the NP supposed to do ha-ha and how they are supposed to function. I just sort of followed around and just did what preceptor did, I was just like try to dress to the same level of casual or more formal than they did and I was kind of just however they try to act toward the stuff I was trying to act toward the staff, try to just mimic whatever they
NP preceptor was more involved in teaching the student

NP preceptors more familiar with the role; MD preceptors expected students to shadow more

Learned more from NP preceptors

Learned compassion from NP preceptor

Never had NP

preceptor; lack of

clarity in preceptors; expectations

NP preceptors modeled role 
were doing.

P14: My preceptors were NPs so they really kind of show you this is what you are looking for, this is what you do, these are the things you want to learn.

Teaching Methods

Approachable

P1:...they had me go home and read, one of my preceptors asked me questions every time I came in and if I did not know the answer she would not tell me, she would have me go back and look it up and that was helpful...

P4: He actually, used to had me do a little project things, he would come up with the topic and then have me to do my research and come back and present that to him, so that was like really helpful with my learning process...

P5:...I had one preceptor that literally on top of my own homework and me working the sole income in the house, he was giving homework assignments and if I did not finish them, I was not to come back the next day, heh, so on top of what my school was requiring,...

P5... she was more focused on making sure that I understood the thought process as to what I was finding...

P13:...3 of them like if I have questions would usually direct me to like look things up which I ... up in my clinical practice all the time ha-ha, so I feel like they helped me to identify where my reading resources are and where I should be looking for things, which in clinical practice is very helpful to me...

P3: When you had a question, yeah yeah, they were very
NP preceptors modeled the role

Teaching by giving assignments and asking student questions

Giving student assignments

Giving student assignments

Explaining the rationale

Helped to direct a student to resources

Approachable and 


\begin{tabular}{|c|c|c|}
\hline and & approachable and they always gave you time to discuss they & encouraging \\
\hline encouraging & like you ask questions and then, they never look at you down, & preceptor \\
\hline Preceptors & you know they if you ask a question, we look it up together, & \\
\hline & sometimes, especially in my adult health, he is always says & \\
\hline & 'great job' (loughs), always encourage you... & \\
\hline & P7:...my women's health preceptor was progressive I would & Preceptor's trust \\
\hline & say, so she really trusted me and allowed me to just do & helped NP's \\
\hline & everything, which upped me my confidence and allowed me & confidence in her \\
\hline & to feel confident with doing my role as a NP on my own... & role \\
\hline & P11: Preceptor that was really good, she was a like also very & Encouraging \\
\hline & approachable and really laid back and she had a you know & preceptor allowed \\
\hline & patient load we were seeing enough patients and she let me do & semi-independence \\
\hline & a lot and encouraged me and was doing evidenced based & \\
\hline & medicine. & \\
\hline & P14: If you had question you can certainly approach them and & Preceptors' \\
\hline & I was fortunate, all of my PNP preceptors were very & approachability \\
\hline & approachable. & facilitated asking \\
\hline
\end{tabular}

Note. Theme II: Pre-graduation: Preceptorship. Preceptor.

Post-graduation: Preceptorship during role transition and learning. When asked about learning from their preceptors during the transition period after graduation, participants identified two major categories of Novice NP' learning and preceptors' teaching.

Novice NPs' learning. Within this code, the major element revealed by participants was self-directed learning. Self-directed learning element included components of utilizing electronic resources, learning through asking questions, and learning by role immersion (see Table 8). 
Self-directed learning. Participants reported that finding and using appropriate resources was an essential part of their role learning during their role transition. More specifically, electronic and paper resources were used to look up and consult the current treatment and management guidelines. Resources noted by participants included EMR, UptoDate database, Lexicomp application, Epocrates, other phone applications, textbooks, YouTube videos, and guidelines. EMR was reported to be used expensively to facilitate patient care decisions at the point of care.

- Namely, EMR functions, such as drug information were used by participants, "P5: A person can have such a long list of medications, that you are relying on EMR to look for interactions,"

- EMR template options were used to learn and improve the appropriate patient documentation techniques, "P10: The EMR system, I set up a lot of templates to help me with my documentation,"

- Pt previous management available through the EMR was used to familiarize self with past treatments and management to make necessary management decisions, "P6: Go back in your chart and see what else had been done because I can see all of their same medical records for years and a lot of my patients had been coming to the clinic for a long time;"

- EMR embedded treatment protocols were reported to be helpful in providing a "safety net" for making treatment decisions in a fast pace clinical environment, 
"P5: They have the guidelines laid out for you; I feel it is what I would ultimately be picking anyways,"

- EMR communication functions were utilized by novice NPs to communicate with other providers, including collaborating physicians when seeking assistance.

Extensive use of EMR after graduation was reported by most; however, the use of EMR was noted to be restricted during participants attending NP programs. This lack of documentation experience translated into a more difficult time adjusting after graduation.

In addition to extensive use of EMR functions, participants reported using many visual instructions or videos found online to self-educate on various procedures and assessment techniques, "P16: I do a lot of like you can get all kinds of stuff on YouTube, made by like medical colleges.” Though data showed that prior graduation, participants were learning extensively through observation and some active learning, after graduation, self-educating though the use of resources was found to be the primary source of information for practicing novice NPs.

In addition to using resources, participants reported learning through asking other professionals around them questions, "P6: ...if I needed to collaborate or ask the questions, I could ask my physician or other provider on site for assistance." Though several participants reported that they did not have other providers available to ask questions regarding patient care, many were able to reach out to those who worked alongside with them. Results showed that asking questions was one of the most important ways of learning new information, both before and after graduation. However, both prior and after graduation, providers did not have any 
carved out time to teach NP students or novice NPs. Lack of providers' time limited the number of learning opportunities novice NPs had.

Participants reported that embracing their new role and being immersed into the clinical environment helped them to learn, "P6:... as far as you transitioning as soon as you can embrace the role the better; actually live it and breath it; I think you are better off so you have that mindset to be able to embrace your role." However, limited clinical opportunities reduced NPs' abilities to "live and breathe" their new role prior the graduation. Those participants, who had other NPs in close proximity within their new work environment, reported a much better experience with learning their new role. Overall, using various resources including consulting with other providers was found to be an essential component of participants learning their new role during their transition to practice.

Table 8

Theme II: Post-graduation: Preceptorship During Role Transition and Learning. Novice NPs' Learning. SelfDirected Learning.

\begin{tabular}{lll}
\hline $\begin{array}{l}\text { Subtheme/Code } \\
\text { Novice NPs' }\end{array}$ & \multicolumn{1}{c}{ Participant's Comment } & Summary \\
Learning/ & P1: But most of the time, most of the things that I have & Mostly self-learned using \\
\hline Self-Directed & learned and that I do it is because I looked it up and I & resources \\
Learning & researched it myself....we had resources available to us at & \\
& work, such as UptoDate, so I used that a lot... & \\
& & \\
& P3: And then after first year of no job and not finding a job, & A lot of self-learning, \\
& eventually I landed a job, super excited (heh), yeah, I am & appreciate the job \\
& super excited, so I just work as hard as I can to be you know &
\end{tabular}


as competent trying to look up a lot of you know read, look

up, make sure what I did is up-to-date.

P6: ...my organization allowed access to UptoDate, so I Uses UptoDate used that as resources...

P5: ...you kind of relying on EMR because sometimes you

Used EMR for drug-drug are just like you know a person can have such a long list of interactions medications, that you relying on EMR to look for interactions...

P5: ...they have the guidelines laid out for you; I feel it is Using EMR embedded what I would ultimately picking anyways... treatment guidelines P6: ...go back in your chart and see what else had been done because I can see all of their same medical records for years and a lot of my patients had been coming to the clinic for a long time...things that I did not know as well or see things I was not sure about I just made sure I looked them up or you know had access to my resources...

P9: ... anytime I had questions about diagnosis, like what in Using Online resources the world is going on with this patient, I had to... my resources online... and then figure it out.

P7: ...I bought a derm. book, I bought Epocrates, I use Using Dermatology text, UptoDate...

Epocrates, and UptoDate

EMR templates

P10: ...the EMR system I set up a lot of templates to help me with my documentation...

P10: ...I try to do relatively independent as much as I can, I Only asks questions 
might check UptoDate, drug references, if I am not if I can't find the answer within I would say within 5 minutes...

P12: ...this job it is a lot different, there is nothing in the computer to tell you to dose or medication, you know what I mean right, so I do kind of feel like I am looking thigs up constantly...I look stuff up on Up-to-date...

P12: ...I felt comfortable, I had a lot of protocols for things so even if you don't remember the dose of antibiotic that you are supposed to prescribe fore strep it is like in the computer when you click on strep dx so kind of like fail prove like...

P14: ...there were things that I have never seen, never even experienced even in my rotation that would come in and initially had to call her and actually I did not call her in those cases because most of the time never responded and ... it was Up-to-date, Google... if they don't have up-to-date I don't know what I will do ... so Up-to-date and I had I paid for an App Lexicomp app for peds patients that had like a little bit of a like a 5 minute consult on it...

P14: ...fortunately for me the patients that we were seeing were the patients that come to his practice quite a bit, so there was history that I could go look at and kind of guess what this patient's story because you only have 15 minutes for the patient, you are not going to get a full picture and you really want to get it...

P14: ... sometimes I will type in in E notes to the because it was the sole practice, ...I will type in the e mail and I may hear about from her, or not...so even though he was not physically there, there was a system in place where every when unable to find answers independently Looks up UptoDate a lot Used "fail prove" EMR treatment protocols

Used UptoDate, Lexicomp, Google, five minute consult,.

Used patient history to make patient care decisions

Used e-note in EMR and e-mail to communicate with collaborator regarding patient care 
patient I saw went into a bucket for him and then he will review those patients... He looked at my notes at least, if he did not agree, he would come and say I think maybe we should call the patient back and do this but very rarely.

P15: ...so that is one of the expenses that I use my allotment is Up-to-date and I use, a lot of my materials that I use to prep for work was like some of the diabetic organizations have the guidelines, a lot pf pocket cards and just things that I find print off of the internet.

P16: I ended up buying a specific book that helped me with that, like if this lab is off, this is the next thing that you order. ...so I paid for Epocrates, that was super helpful... sometimes if I could not find it on there I would use Up-todate...I had my ...book if there was something...

P16: ...I do a lot of like you can get all kinds of stuff on YouTube, made by like medical colleges and what not...

Learning through P2: ... if I had questions about complex patients I would asking questions.
Used UptoDate, pocket cards, guidelines
Purchased Lab book, used Epocrates and UptoDate

Used Youtube extensively

Asked all involved in patient care worker to see how to discharge this kid inpatient psychiatric care, or,...you know just the resources to try to get in touch with people but ye, it was just you know whoever was involve, just keep asking around and till you find the right people.

P6: ...if I needed to collaborate or ask the questions, I could ask my physician or other provider on site for assistance.

P7: ...I actually kind of reached back to some of my Reached back to formal preceptors that I had before...

Asked questions MD and other providers preceptors for help 
P13: ...sometimes I will call the other physicians or people from other specialties who see patients if there is something that is going on that I don't understand or I don't know how it is impacting what I am doing...

Embracing the new role through immersion
$\mathrm{P} 2 \ldots$ just kind of like learn by being immersed into the environment.

P6: ... as far as you transitioning as soon as you can embrace the role the better actually live it and breath it I think you are better off...so you have that mindset to be able to embrace your role.
Called other providers involved in patient care for clarifications

Learning by immersion in the environment

Living and breathing the role

Note. Theme II: Post-graduation: Novice NPs’ Learning. Self-Directed Learning.

Preceptors' teaching. Participants reported that on the job training often involved passive shadowing other providers (see Table 9). Participants reported that "hands-off" experiences or simply shadowing the providers hindered their learning of a new role,

P11: My first job, he gave me about like a month, where I shadowed another provider and then he started... my patient load and I remember feeling very like unprepared but I did ok...he was not, he was very hands off, he never discussed clients unless I went to him specifically, we never had like a set time to sit down and discuss cases.

Participants reported that discussing patient cases was rarely used by their preceptors but if it were, it was helpful,

P11: ...one of my preceptors in this current job... she would try when we have time and we would sit down and kind of talk about stuff and so that was nice and I was trying to take notes as we talked about different diagnosis... 
At the same time, participants reported that active involvement in the decision making process during the onboarding time facilitated their learning of NP skills,

P1: I was able to watch one of the NPs do incision and drainage on an adult, because I asked, can I go in with you, and she was fine with it, ... she allowed me to go in and watch and I watched and I have done them on my own on an adult. For kids, it is a little bit different (heh) and I did seek help from one of the preceptors; she did not go in the room with me but she did walk me through what to do and how she does it and I was...now I do them all the time...

Many participants reported experiences with their preceptors who were not willing or able to take active part in NPs' training when onboarding,

P7: ...my collaborating physician I was ... who did not really, I mean he helped me when I needed to but for the most part he just let me do my thing...

P15: ...they had me followed him for a few weeks just and I was just standing observing his surgery and he had a resident with him so the resident was always up near the OR people, so I was usually standing off back in the background, so I did not really see and he was always interacting with the resident and rest of the OR staff and anesthesia, so I really did not learn anything from going into the OR with him, and then as far as in the clinic I just shadowed him into exam room...

Hands-on experiences along with participants' active involvement during the onboarding time when transitioning to NP practice were reported the most helpful in leaning the role. 
Participants also revealed that if their employer required them to perform certain procedures or skills they have not previously learned or developed in NP school, they had to selfinitiate learning these skills and learn them "on the fly" after or during the on the job orientation, which was not always provided, "P11: See one, do one, teach one and that has been fairly effective, learning how to do joint injection, suturing, trigger points injections, and incision." However, if participants' jobs did not require them to perform certain procedures or NP skills, these skills would be left unlearned: "P3: No I still cannot do the suturing, incision and drainage, it is just we are not required to do that, and if there is incision and drainage, there is... we always consult." Results showed that NPs graduated without some skills that are necessary to practice, which led to their reliance on the employer upon the graduation; however, participants reported that their experiences with most employers showed that their employers expected them to enter practice being fully trained. Thus, participants had to be thrown "to the wolves" and learn the missing skills "on the fly" when able. Participants reported attempting to learn these skills from already busy providers who worked alongside with them. Some participants reported this way of learning as being successful; it was reported as helpful when preceptors were approachable, available, and willing to help,

P4: I feel like I can go to her and ask her anything and she is not going to like brush me off and all. Like I almost feel like I am in that precepting stage because I can just go to her, you know, at any time like she can be busy with the million patients and I can say hey step in for a minute, take a look at this with me, and she does, she does... 
However, some participants reported that their preceptors were not willing to teach. Whereas, others reported learning all they could alone because they did not have anyone else to help them learn on site.

Table 9

Theme II: Post-graduation: Preceptorship During Role Transition and Learning. Preceptors' Teaching.

\begin{tabular}{lll}
\hline Subtheme/ & Participant's Comment & Summary \\
Code & &
\end{tabular}

Preceptors'

Teaching/

\begin{tabular}{|c|c|c|}
\hline Preceptors' & P1: ...I was able to watch one of the NPs do I\&D on a an adult, because & Hands on teaching: \\
\hline \multirow[t]{6}{*}{ Teaching } & I asked, can I go in with you, and she was fine with it, ... she allowed me & See one, do one \\
\hline & to go in and watch and I watched and I have done them on my own on & \\
\hline & an adult. For kids it is a little bit different (heh) and I did seek help from & \\
\hline & one of the preceptors; she did not go in the room with me but she did & \\
\hline & walk me through what to do and how she does it and I was...now I do & \\
\hline & them all the time... & \\
\hline
\end{tabular}

P3: No, no I still cannot do the suturing, I\&D, it is just we are not No training to do required to do that, and if there is $I \& D$ there is we always consult... procedures since job does not require P4: ... so I don't do colposcopies, I think you have to be certified in that, I No training to do also don't do meds like Nexplanon and IUDs... if I was to move you procedures since know to go somewhere ..ever go to another clinical working in women's job does not clinic and that clinic probably would expect me to be able to do those require procedures so. Something to think about.

P4: ... I feel like I can go to her and ask her anything and she is not going Approachable and to like brush me off and all. Like I almost feel like I am in that helpful preceptor precepting stage because I can just go to her, you know, at any time like 
she can be busy with the million patients and I can say hey step in for a minute, take a look at this with me, and she does, she does...

P6: So did you have people coming in with laceration or doing sutures or any of that? No we did not do a lot of that, I still, this is a skill I want, I am going to have to branch out may be do training someplace else because we just don't have a lot of that.

P6: ... at one point I asked for time with my site preceptor to go over labs. So I was there a little bit of time one day a week for may be 30 minutes... we both had time allocated to do that, questions about lab work, that was difficult as a new provider because at clinical site you are not there to see the follow up...

P7: ...my collaborating physician I was ... who did not really I mean he helped me when I needed to but for the most part he just let me do my thing...

P8: ... it is like if I have never even seen them done before I always watch them the 1 st time, am and then do it with someone the 2 nd time and again with someone... So it sounds like most of the procedures that you are doing now, you have learned on the job? Yes, all of them.

P8: ... so his style was to kind of to come back in and he is explaining to the parents some information but the way he is explaining it is also directed at me, so he kind of was able to ...where he is teaching me while we were with the patient rather than just talking to me about it in a didactic way in another room and I really liked that because it kind of gives me hands on with learning.

P8: ...I work for a large group but I kind of had 1 physician, the one who like hired me and then I worked with more often than anyone else, so he kind of like an informal kind of mentor, and I would kind like run things
Lacks procedure skills; work does not provide training

Allocated time with preceptor to learn labs

Uninvolved preceptor Was able to learn some procedures on the job via see one-do one strategy

Hands-on preceptor teaching style

Informal preceptor 
by him, check in with him on how I was doing basically overall, because

I would not always work with him every day, but just like have someone to kind of check in with.

P9: Sutures I learned when I was an NP. I had to fly out of town to take the suturing class.

Took a class to

learn how to suture

P10: ...my preceptors were helping me try to learn the I guess the role a

Learning

little bit more along with delegation...to learn how to use the nurse and

delegation

staff or my medtech whoever was delegated that day to be my am

support staff,

P11: My 1st job he gave me about like a month, where I shadowed

Hands off

another provider and then he started... my patient load and I remember

preceptor style

feeling very like unprepared but I did ok...he was not he was very hands

off, he never discussed clients unless I went to him specifically, we never

had like a set time to sit down and discuss cases. And it ended up being

ok, I kind of felt I taught myself a lot...

P11: ...through job training, is one of the I would say that the doctors

See one, do one

... their medical school model, see one, do one, teach one and that has

been fairly effective, learning how to do joint injection, suturing, trigger

points injections, and incision and

drainage, removal of an ingrown tail nail, that sorts of thing, digital

blocks, local blocks...

P11: ...one of my preceptors in this current job...she would try when we

Preceptor teaches

have time and we would sit down and kind of talk about stuff and so that

through

was nice and I was trying to take notes as we talked about different $\mathrm{dx}$...

discussions

P14: the unfortunate part that was the feedback we gave when we were

Learning from

leaving school is that procedures, even if in PC you can't assume that you

residents

are going to PC clinic and there won't be kind of just a couple stiches... 
So how did you learn all those things... On the fly... How was that?

Terrifying. I did a little bit of so the very first suture I did and attending

was there, o you never sutured? Mm, and you know residents can't

graduate without having those...

P15: ...they had me followed him for a few weeks just and I was just

Hands off

standing observing his surgery and he had a resident with him so the

preceptor's style

resident was always up near the OR people so I was usually standing off

back in the background so I did not really see and he was always

interacting with the resident and rest of the OR staff and anesthesia so I

really did not learn anything from going into the OR with him and then

as far as in the clinic I just shadowed him into exam room...

P16: So she would like so the 1st time I did a mole removal was actually

Preceptor walked

was on one of our MA, so doctor being literally just walks me

NP through

through... as I did it...

procedures while

she was doing it

P16: ...I also had to do all her patients' labs as well like I was

Learning labs on

responsible for all the tests ordered...we did not get a lot of teaching in

her own

school about labs and I like, that was, I felt like I really struggled with

that at first...

Note. Theme II: Preceptors' Teaching.

\section{Theme III: Learning to Care for Complex Patients}

In the third interview question, participants were asked about their experiences with learning the more complex NP skills while transitioning to their new NP role both during them attending NP program and after graduation.

Pre-graduation: Learning to Care for Complex Patients. When asked about the transition time prior the graduation, participants identified one major category of learning 
complexities patients (see Table 10). Participants reported difficulties with obtaining necessary experiences with complex patients mainly due to the lack of control over the chosen clinical sites, "P16: I think it was like completely random, because you were just lucky to get what you could get and I think I got lucky.” Participants' experiences were limited by the patient care opportunities that were offered by those clinical sites that participants were able to find and secure. Thus, difficulties in finding and securing clinical sites combined with limited clinical hours, caused participants to settle for those sites and preceptors that would agree to take them; "P13: If you are going to have your clinical on Thursdays from 8 am to $5 \mathrm{pm}$, you are sort of limited to whatever patients come in on the Thursday from 8 am to 5 pm..."

This inability to select sites based on quality was reported to result in random experiences, which often meant that participants took care of more simple cases. Because of this lack of exposure to complexities of a real world practice, participants stated that when they transitioned to their practice, they felt significant discomfort and lack of necessary knowledge and skills to take care of their complex clients, "P8: I would say for the most part in school I was concentrating on simple, and then in practice I am still not super comfortable caring for super complex patients..." Participants reported that lack of training in providing complex medical patient care affected their transition to practice after graduation when they were presented with everyday complex and challenging patient care opportunities. Mismatch between the level of training received in school and the demands of current health care practice was reported to be the cause of difficult NP role transition. Data analysis showed that it is possible that NPs' perceptions of caring for predominately simple cases when in NP school were related to their 
infrequent hours at the clinics, inability to follow up on their patients, frequent change in preceptors, and inability to practice autonomous decision making skill when diagnosing and prescribing. It is also possible that NPs preceptors' time only allowed addressing simpler cases with NP students. Additionally, frequent preceptor change may have been related to preceptors' inability to learn NP students' learning needs, which resulted in more random patient care opportunities.

Table 10

Theme III: Pre-graduation: Transitioning to Complex Pts and Learning. Learning Complexities.

\begin{tabular}{|c|c|c|}
\hline $\begin{array}{l}\text { Subtheme/ } \\
\text { Code/ } \\
\text { Learning } \\
\text { Complexities }\end{array}$ & Participant's Comment & Summary \\
\hline \multirow[t]{3}{*}{$\begin{array}{l}\text { You get what } \\
\text { you get }\end{array}$} & $\begin{array}{l}\text { P3: ... even though we only see eight patient, but each of them had } \\
\text { multiple problems, they don't come in a lot, but they come in they have } \\
\text { all kinds of issues and it is helpful because for them you have to refer } \\
\text { them to like to all the different places... }\end{array}$ & $\begin{array}{l}\text { One clinical } \\
\text { rotation had } \\
\text { excellent complex } \\
\text { patient population, } \\
\text { which was helpful } \\
\text { to a student. }\end{array}$ \\
\hline & $\begin{array}{l}\text { P4: ...I feel like our program... I don't really think they prepared us to } \\
\text { care for complex patients. }\end{array}$ & $\begin{array}{l}\text { Unprepared to } \\
\text { care for complex } \\
\text { patients }\end{array}$ \\
\hline & $\begin{array}{l}\text { P7: ...one thing about when in school I did not feel as much pressure } \\
\text { when managing the complex patients... my focus and energy on a } \\
\text { specific patient so focus more on complex patient... whereas now I } \\
\text { have to focus on all my patients and all my test results and all my } \\
\text { extra... lot of small things that that were not there when I was a }\end{array}$ & $\begin{array}{l}\text { School clinical } \\
\text { experiences did } \\
\text { not match the } \\
\text { complex reality } \\
\text { which included }\end{array}$ \\
\hline
\end{tabular}


everything and it did not really matter if they were simple or complex

responsibilities

patients, whereas other preceptors would have me focus on 1 patient in

hour...

P8: ...I would say for the most part in school I was concentrating on

Mostly simple

simple, and then in practice I am still not super comfortable caring for

patients at school, super complex patients...

uncomfortable

with complex now

P8:... they can't really control what patients are going to walk into the

Can't be picky

clinic, especially because you find your own preceptors so that is very

with sites, so you

difficult to find your own preceptors so you know they are not exactly being like that be some complexity of the population or anything...

get whatever

patients you get

P11: I would say they were just very basic, I don't remember seeing anything super complex... it was more like a lot of well visits... I honestly feel like in school I did not see a lot of complex stuff. I feel like we had a lot of basic stuff and then like I don't even remember seeing much asthma, which can be really complex... so now that I am getting out there and I am trying to do gen peds I am like OMG I don't know what to do, you know... now that I am out, I feel like I am seeing a lot more complex stuff than I did in school.

P11: ... the most I ever saw might have been 12 but that seems really high, I don't think I was seeing that many, to be honest with you.

Seeing low patient volume at school

P13: ... it just depends on which I mean if you are going to have your

Pt care clinical on Thursdays from 8 am to 5 pm your sort of limited to opportunity types whatever patients come in on the Thur. from 8 am to $5 \mathrm{pm} .$. are limited by $\mathrm{Np}$ student's schedule

P13: ... one of my clinicals I felt like was not super helpful, it was like a Pt care walk in clinic... which I mean that is like a type of setting you can work opportunity types 
in but the complexity of patients was very low... the other ones, I did

half of semester with palliative and half of semester with cardiology; I

felt like it was much more helpful... somewhere in between like 4 and

6 a day probably or 4 and 8 .

P16: So I tried to choose patients that had some difficult things, so that I can learn from people that were really good at that... I think it was like completely random, because you were just lucky to get what you could get and I think I got lucky. are limited by the

setting

"You are lucky to

get what you

could."

Note. Theme III. Learning Complexities.

Post-graduation: Learning to Care for Complex Patients. When asked about learning the complexities of patient care during the transition time after graduation, participants identified one major category of complexities of the real world. The major code revealed by participants was related to time management of patient care (see Table 11). More specifically, participants reported struggling with learning combining high patient volume with providing quality care,

P7: A lot of patients will come in with the chief complaint will say pain but then they also want to talk about their irregular period and their rash that they had, so just trying to learn how to focus on one thing per visit.

Performing new duties, adjusting to new responsibilities, and taking on complex patients without previous exposure to some diagnoses and treatment in short 15 minutes slot appointments, brought feelings of fear of making a mistake. Participants reported that it was helpful to have longer appointments in the beginning to allow for learning. However, there was no consistency in how this strategy was used among different practices. Some reported that they were allowed this partial schedule for few days or weeks and some-for several months. Several participants 
reported having to do full patient schedule right after the credentialing process was completed.

Other codes included various complexities of patient care participants were faced with after graduation, including underserved population, high risk patients, multiple comorbidities, noncompliant patients, polypharmacy, referrals, new or less known by NPs patients, various types of patient visits, and taking work home (see Table 4.11). Participants revealed referring complex patients to next level of care or to their collaborators when unable to handle and the referral is available.

P16: So complex patients is what I really struggled on, when I first became an NP, and I felt especially like so in our office they were scheduled for a $30 \mathrm{~min}$ visit, so by the time they were pulled in by the MA, sometimes you only have like 15 minutes to see them, chronic care patient with uncontrolled DM, uncontrolled HTN, you know all these whole gamut of stuff and I got like 15 minutes to figure out what needs to be done, how to change their meds, it was very overwhelming... all of the sudden you have to work through this really complicated person that is one 6 different DM meds and 4-5 different BP medicines and all these things and stuff is not working and labs are all junking you got to figure out, ok what am I going to add on to this and then you throw in the fact that the patient cannot afford their medicine as it is, or the insurance won't pay for this or this, but they will pay for this and trying to like to like cram all that in, assess the patient, talk to them, figure out everything to order and change in like 15 minutes, it was very very overwhelming at first... 
Participants reported a significant disconnect between what they were prepared for by their programs and what they actually had to do when starting practicing. Taking care of very sick patients without having appropriate support right after graduation was reported as challenging, overwhelming, and scary.

Participants reported being expected to "hit the floor running" or to take care of complex patients in a short amount of appointment times from "the get go," calling it "trial by fire," "P1: They do not really understand, they think that if you got FNP behind your name you should be able...things that we do here.” Some participants reported minimal support from their collaborators when they were requesting help with complicated cases, "P14: There really was not any consistency as to when or why she chose to calling..." Participants noted that employers often expected them to be fully trained to deal with complexities of patient care process right after graduation. However, without rigorous training participants often found themselves full of self-doubt.

Table 11

Theme III: Transitioning to Complex Pts and Learning Post-Graduation: Complexities of a Real World

\begin{tabular}{lll}
\hline $\begin{array}{l}\text { Complexities } \\
\text { of a real world }\end{array}$ & Participant's Comment & Summary \\
\hline Complexities & P1: ...they felt that because I am an FNP, I should be able to do those & Unrealistic \\
of a real world & things, they are not clinical, so they do not really understand, they think & employer's \\
& that if you got FNP behind your name you should be able... things that & expectations of NP \\
& we do here. Even they expected one provider float to OB one time; she & being fully \\
& & prepared to \\
& & practice \\
\end{tabular}


without additional

training

P1: ...making sure that medications do not interact with each other

Polypharmacy because they are on like a long list of medications so that part was patients require a really hard because I took up a lot of time to kind of do a research and lot of medication looking things up to make sure that I was covering all my basis as far looking up as screenings for certain patients...

P3: ...most of the time the stressful is really come from you have to see Time management a certain patient in limited time period...

P3: Sometimes when you round, when you discharge patient there are Comorbidities many difficulties because the patient you know all kinds of issues...

P3: ...time consuming complex patients, I will see the patients in the hospital, they are all pretty complex, they come in with multiple Time management complaints, and they a lot of them, they really don't have resources...

P4: ...working in OBgyn, which is has a lot of liability to it, a lot of High risk/referrals times when I do have complex patients you know I do have to refer to my collaborating physician, so a lot of complex she handles.

P5: ...you get to a point where you kind of relying on EMR because Polypharmacy sometimes you are just like you know a person can have such a long list of medications, that you relying on EMR to look for interactions am, between any of the medications that a person is on but even still at that point unless something you know like super well known, you may not even know if there is an interaction with something because there are just so many things and so it can be sometimes even scary when you are the person to be adding a medication to someone who is already taking so many... 
P5: ...I was there for a week and I ended up leaving just because I recognize immediately that that was not an environment I wanted to work in...

P6: ...that is important that you have your encounters, your labs done and getting your feedback to your patients properly and then the whole other aspect of this that you don't learn at school, that comes up that having to do like FMLA form, so having to do different kinds of visits like pre-surgical visits or more focused problem visits that you don't always feel like training...

P6: ....I see a lot multiple comorbidities, and then uninsured and noncompliant...

P7: Ye so this definitely was challenging part of my transition being provider ... a lot of patients will come in with the chief complaint will say pain but then they also want to talk about their irregular period and their rash that they had so just trying to learn how to focus on 1 thing per visit, I am learning to always incorporate wellness into every visit and it can be really challenging or patients come in with multiple problems...

P8:... in practice I am still not super comfortable caring for super complex patients, so patients with multiple medical conditions genetic disorders, things like that I sometimes have when I am seeing the really complex patient come on my schedule, there have been a few times where I have asked them to change them to physician schedule because I don't think it is appropriate.

P10: ...there is a lot less I feel safe about, there is a lot less of medications I feel safe about issuing if I don't know if somebody comes in and they are $85 \mathrm{I}$ don't know what their renal function is...
Having to leave

jobs

Various visit types

Time management

\author{
Complex \\ diagnoses
}

Not having

complete patent

information 
available

P11: ...I have not seen a high volume of patients because they are being very kind to me but I know that that will not stay the case and eventually I could see up to 27 patients which sounds horrendous to me and I don't know how I would ever do that...

P11: ... sometimes you have to chart when you get home and sometimes you have to like you know follow up on lab results when you get home because the culture comes back negative or positive and you have to do something with it...

P12: ...you have a lot of patients waiting and you would don't have a lot of downtime, so that was a little bit stressful and you are by yourself there...

P12: I did not really have a lot of complex patients at the retail or UC I mean if I did I just referred them all but at the job I am now there is a lot really sick patients, like they get a patient who has cirrhosis, varices, and you know ascites that need paracentesis, like and that is still a little bit uncomfortable for me...

P12: ... anything that seemed above my head I would refer out...

P14: And she, there were times there were patients I really thought she needed to see or discuss with me, I would have the front desk call her in hopes may be at some point she would want to touch base with them and that way it will catch me or wise versa and some days I was ..it was and there really was not any consistency as to when or why she chose to calling...

P14: Prescribing was merely just kind of just on the go ... which has been one of the toughest things coming in after graduation and having
Time management

/ Pt volume

Taking work home

Time management

Difficult diagnoses

Referring out

Difficulties with reaching collaborator to discuss complex cases

Prescribing 
to do...

P15: ...I am not still at a comfort level with all of that especially with diabetes and so forth, it is challenging...

P15: ...well that is still kind of ongoing challenge for me just because especially where I work the patients are pretty complex, because some of them may be have not had medical care for several years...

P16: So complex patients is what I really struggled on, when I first became an NP, and I felt especially like so in our office they were scheduled for a 30 min visit, so by the time they were pulled in by the MA, sometimes you only have like 15 minutes to see them, chronic care patient with uncontrolled DM, uncontrolled HTN, you know all these whole gamut of stuff and I got like 15 minutes to figure out what needs to be done, how to change their meds, it was very overwhelming... all of the sudden you have to work through this really complicated person that is one 6 different DM meds and 4-5 different BP medicines and all these things and stuff is not working and labs are all junking you got to figure out, ok what am I going to add on to this and then you through in the fact that the patient cannot afford their medicine as it is, or the insurance won't pay for this or this, but they will pay for this and trying to like to like cram all that in, assess the patient, talk to them, figure out everything to order and change in like 15 minutes, it was very, very overwhelming at first...
Treatment plans

Underserved

Time management

Note. Theme III: Complexities of a real world

\section{Theme IV: Learning in clinical environment}

In the fourth interview question participants were asked about their experiences with learning in a context of their clinical environment while transitioning to their new NP role both during them attending NP program and after graduation. 
Pre-graduation: Learning in clinical environment. When asked about the transition time prior the graduation and its support through the clinical environment, participants identified two major categories, space and relationships with people (see Table 12).

Space. The one major code of the space category reported by participants was clinical sites. This code consisted of the clinical sites and clinical sites quality components, which emerged from the interview data.

Finding clinical sites. First, it was found that many participants experienced significant difficulty in finding and securing clinical sites. Clinical site competition with other schools (including medical, PA, other NP schools, and online schools) was reported as one of the frequent barriers to securing a site,

P10: So as far as selecting sites, I certainly I wish that I had the ability to be ... a bit more selective; however... and it was very difficult to find clinical sites, I did .... little math and two hours' drive in any direction, there were 12 NP programs, three medical schools and three PA schools so getting the sites was almost impossible.

Participants specified that many sites had affiliations with the local schools, making it more difficult to secure a site if the respondent was enrolled in an online program,

P16: That was the worst part of graduate school, was finding the clinical sites because different hospitals and primary care locations and what not affiliated with different universities... so it was very limited the spots that you can get and so then you are fighting with other people in online universities to get spots.

These challenges in finding clinical sites often lead to students spending a lot of time and 
resources on finding the sites. Some participants reported having some of their clinical experiences at the remotely located sites, which in some instances were located in a different state, leading to additional expenses and travel time, "P5:...I ended up hiring this one women she said she was clinical matchmaker...”

While the burden of finding sites was mostly on participants, some reported that their schools found sites for them; "P6: My school made all the clinical placements..." Additionally, in some cases participants reported that schools allowed them to navigate the process of site selection to match the sites with the preferred experiences.

P14: ...my program director would ask what kind of experience so the semester prior, do you want more primary care, do you want more specialty and I kind of always wanted specialty, so I got a lot of my rotations were specialty days...

Regardless of whose responsibility was to find a site, school's or a student's, some, participants stated that occasional adjustments of changing sites after the start of the rotation, took place per their requests when the initially chosen site appeared to be inadequate or did not provide the student with the necessary experiences.

P2:... if I start having an issue and I felt like I was not learning in a clinical site, I very like clearly told whoever was directing that class and then a lot of times they would go and allow me to, you know make accommodations...

In summary, most participants $(68.8 \%(\mathrm{n}=11))$ reported that it was their responsibility to find and secure clinical sites. This experience was reported as one of the most challenging aspects of participants' NP program for many. Difficulties with finding clinical sites resulted in settling for 
any sites and preceptors affecting the quality of participants' clinical experiences in school.

Clinical sites quality. Because of not finding a clinical site often meant not being able to proceed in the program, participants' priority was to secure any available site. Participants reported having to experience "last minute scrambles" when trying to secure clinical sites; they reported that they were unable to be selective in terms of the sites' quality.

P8: There was no way of telling if they were going be good teacher... You basically had to cold call people.

"P5: ...my first two rotations it was kind of a last minute scramble, and I was literally, it was a matter, if I don't do this rotation with these people, I will not graduate...

$\mathrm{P} 12 \ldots$ a lot of people were just scrambling it was just random whoever would take me, is what it was.

Significant variation among the sites and preceptors was reported by participants, including those who had their sites selected for them by the school. For example, participants reported that some preceptors followed the guidelines and some did not, which was confusing to students.

P11: ... one of them was not very good because the preceptor also had questionable like clinical guidelines she was following or lack thereof. She did things not always by the book and then she also did not have a very high patient load...

Additionally, some preceptors had to work at multiple sites and locations and the students were "dragging" behind them; participants reported that these types of clinical environments were unstable and unsuitable for their learning.

P5: ...first clinical that I was at that it was MD he had his own private practice, he also 
rounded to two different hospitals, as a hospitalist and then he was a medical director for nursing homes, rehab facilities, and he was just bouncing around to all of these different places and, ultimately, I felt that what my schooling was for was just mostly for the clinic setting, so I did not really feel like I got a good idea what was around because we were bouncing around so much...

P7: ...it was a really busy environment, pretty dynamic environment because we were in multiple places in one day. So I never really felt any stability in that environment.

These frequent changes of sites and preceptors were reported to effect preceptors' ability to trust students with patient care; participants also reported increase in self-doubt and being unsure of what was expected of them by different preceptors. Participants noted that as a result of frequent changes in sites and being unfamiliar with preceptors, they had experienced more shadowing time than expected and in some instances they were only allowed to shadow during some of their clinical rotations. Lack of hands on training opportunities was frequently reported by participants. Participants noted that these unmet learning needs were most noticeable after graduation when they transitioned into practice. Participants noted that their employers often expected them to be fully trained. The mismatch between the schools' training and employers' expectations appeared to be causing participants a high level of discomfort and anxiety as they were starting to practice.

Though participants reported that it was hard to frequently change from a preceptor to a preceptor, they reported that they benefited from these variations having an opportunity to observe different providers' models as well as having to be exposed to various clinical settings. 
This was noted to be helpful with participants' job decisions of specialties or practice settings after graduation.

The type and quantity of patient care opportunities also varied greatly among the sites. Participants stated that some sites did not offer the necessary patient care opportunities to meet their learning needs. Furthermore, participants reported that their clinical hours usually allowed for approximately one or two days a week of clinical time, which did not allow a student to follow up on the patients they previously saw or laboratory tests they ordered.

P11: ...I got basically one day a week, it was 420 house total. And I think that the minimum standard and one day a week is not much and it does not give you time to see follow up or anything, so it is just felt like very unprepared when I came out to actually do ... peds.

Most Participants reported struggling with managing required patient volume, interpreting laboratory results, and performing common office procedures after graduation. These unmet learning needs were attributed to not having the necessary clinical opportunities at school.

It was not the aim of this study to find out if schools also had difficulties finding and securing clinical sites; however, when analyzing the experiences of those participants who had to find own sites and those who had their schools find sites for them, the participants' descriptions of their experiences of learning in clinical environments were similar. 
Theme IV: Pre-graduation: Transitioning in clinical environment and learning. Space. Clinical Sites. Finding clinical sites and clinical sites quality

\begin{tabular}{|c|c|c|}
\hline $\begin{array}{l}\text { Space/ Finding } \\
\text { clinical sites } \\
\text { and clinical } \\
\text { sites quality }\end{array}$ & Participant's Comment & Summary \\
\hline \multirow[t]{7}{*}{$\begin{array}{l}\text { Clinical Sites/ } \\
\text { Finding clinical } \\
\text { sites }\end{array}$} & $\begin{array}{l}\text { P2: The school found the sites for me, I feel like you get what you ask } \\
\text { for in learning, so if there was something... if I start having an issue and } \\
\text { I felt like I was not learning in a clinical site, I very like clearly told } \\
\text { whoever was directing that class and then a lot of times they would go } \\
\text { and allow me to am, you know make accommodations... }\end{array}$ & $\begin{array}{l}\text { School found } \\
\text { sites; student } \\
\text { needed changes }\end{array}$ \\
\hline & $\begin{array}{l}\text { P4: I was in... City. There is thousands NP students that need clinicals, } \\
\text { ye it was very stressful. }\end{array}$ & $\begin{array}{l}\text { Clinical site } \\
\text { competition }\end{array}$ \\
\hline & $\begin{array}{l}\text { P5: In most programs you are responsible for finding you own preceptor } \\
\text { so the variation between you know who you are getting sometimes I } \\
\text { mean it is very huge difference in how each provider practices. }\end{array}$ & $\begin{array}{l}\text { Variations } \\
\text { among } \\
\text { preceptors }\end{array}$ \\
\hline & $\begin{array}{l}\text { P5: ...I was having to reach out to places even } 3 \text { and } 4 \text { hours away, I } \\
\text { was actually accepted for my first rotation in like an Indian Reservation. } \\
\text { And someone agreed to take me on and that was probably } 3 \text { to } 4 \text { hours } \\
\text { away... }\end{array}$ & $\begin{array}{l}\text { Remote clinical } \\
\text { sites }\end{array}$ \\
\hline & $\begin{array}{l}\text { P5: ...I ended up hiring this one women she said she was clinical } \\
\text { matchmaker... }\end{array}$ & $\begin{array}{l}\text { Extra expenses- } \\
\text { hiring clinical } \\
\text { matchmaker }\end{array}$ \\
\hline & $\begin{array}{l}\text { P6: My school made all the clinical placements so they tried to do it } \\
\text { based on geography so where you live so you won't have to be traveling } \\
\text { too far. }\end{array}$ & $\begin{array}{l}\text { School chose } \\
\text { sites based on } \\
\text { geography }\end{array}$ \\
\hline & P7: ... was in my opinion the most stressful part of my schooling & Clinical site \\
\hline
\end{tabular}


because it was very hard to find people that were willing to precept....

competition

There are a lot of schools in the area that I live...that is pretty

competitive...

P8: So it was just really challenging in terms of where I was, in...

Clinical site

place...a lot of NP programs here so there is a lot of people searching for

competition

clinicals, there is also like health systems certain ones that have agreements with certain school, so they only take students from that school...

P10: So as far as selecting sites, I certainly I wish that I had the ability

Clinical site to be ... a bit more selective; however, I was at the time I was based in competition ....and it was very difficult to find clinical sites, I did .... little math and 2 hours' drive in any direction, there were 12 NP programs, 3 medical schools and am 3 PA schools so getting the sites was almost impossible.

P10: ... it was the up to the 3 hours' drive to one particular site I just Remote clinical stayed a week in town and come home on the weekend and it was sites challenging.

P14: ...my program director would ask what kind of experience so Choosing sites the semester prior, do you want more primary care, do you want more specialty and I kind of always wanted specialty, so I got a lot of my rotations were specialty days...

P16: just calling a ton of people, I will be honest with you, that was the Clinical site worst part of graduate school was finding the clinical sites because competition different hospitals and primary care locations and what not affiliated with different universities...so it was very limited the spots that you can get and so then you are fighting with other people in online universities to get spots...

P16: ... a lot of time was wasted on that; that means wasted time, you Wasted time 
are not learning anything, I am not learning anything form spending

hours on my phone, begging somebody to be my preceptor...

Clinical Sites/

Clinical sites

quality
P2: I was all over the place so to say like what were the experiences

like, they were vastly different every semester and every every single rotation. I did not do anything that was like the next time.

P5: ...first clinical that I was at that it was MD he had his own private practice, he also rounded to 2 different hospitals, as a hospitalist and then he was a medical director for nursing homes, rehab facilities, and he was just bouncing around to all of these different places and ultimately I felt that what my schooling was for was just mostly for the clinic setting so I did not really feel like I got a good idea what was around because we were bouncing around so much...

P5: ...my 1st 2 rotations it was kind of a last minute scramble, and I was literally, it was a matter, if I don't do this rotation with these people, I will not graduate...

P7: ... it was a really busy environment, pretty dynamic environment because we were in multiple places in 1 day. So I never really felt any stability in that environment, whereas my last 3 clinicals I was there like twice a week for full days and I was introduced to all the staff in the clinic, it was nice...

P8: ....it was so hard you pretty much take anyone you could get and there was no way of telling if they were going be good teacher, if they were going to be supportive, if there was going to be a good environment, you basically would take whatever you could get...

P10: ...I wish that I had the ability to be a bit more picky or not necessarily picky, a bit more selective...
Variable clinical

experiences

Inconsistent

Learning

Environments
Inconsistent

Learning

Environments
Settling. No way of telling sites and preceptors' quality prior start

Settling 


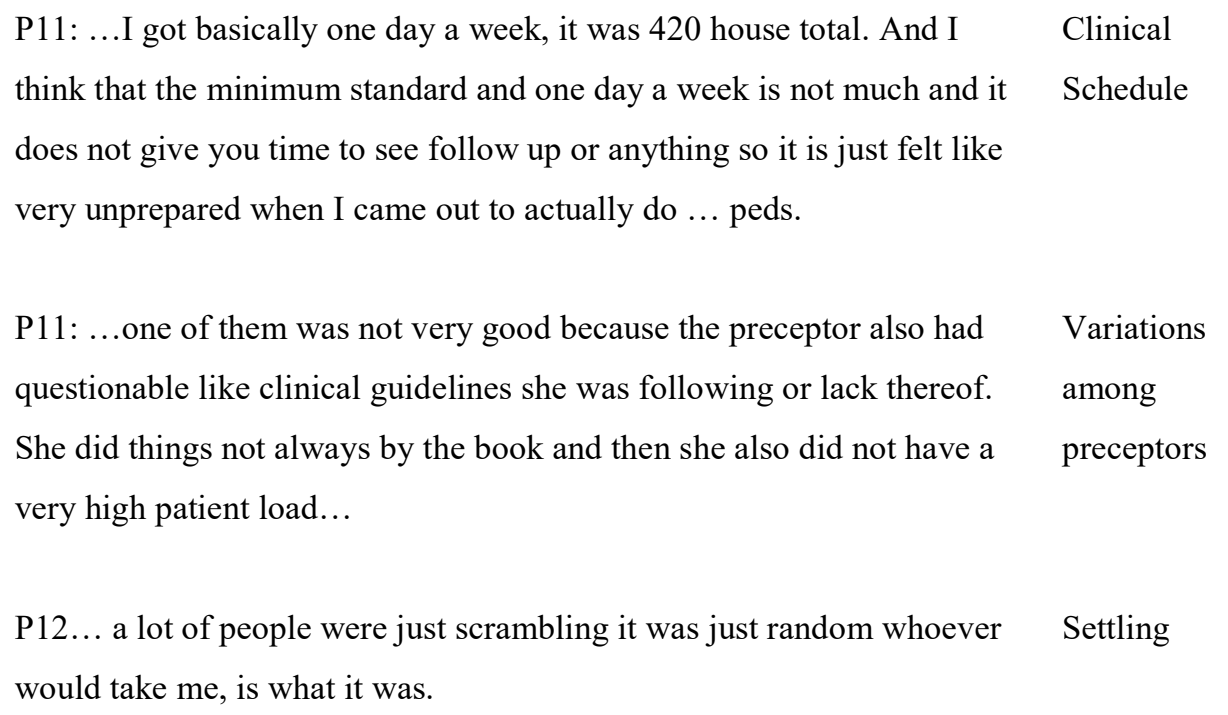

Note. Theme IV. Space. Finding clinical sites and clinical sites quality

Relationships and people. Participants reported "cold calling" and sending mass e-mails to multiple providers in hopes to secure a clinical site. Participants stated that they were often turned down and rejection was taking place right before the clinical start time due to preceptor leaving his or her job or deciding to take another student instead (see Table 13).

P5: I was actually accepted for my first rotation in like an Indian Reservation. And someone agreed to take me on and that was probably three to four hours away from my house and then literally less than two weeks before the rotation started the MD called me and told me, he said I am not going to be able to follow through with your clinical because there is MD student that needs a clinical here so I am sorry, good luck. And I was devastated... 
Begging for clinical sites was reported as one of the most frustrating parts of many participants' experiences in NP school. In fear of not being able to secure any sites in time, participants accepted any site that would agree to take them.

Familiarity with sites and preceptors was reported to be helpful in participants securing the sites as well as learning at the sites. More specifically, previous employment at the site or previous personal or professional relationships with the preceptor was helpful in facilitating participants' learning and communication with the potential sites and preceptors.

P4: I probably have every clinical there is ...names places and locations...but a lot of the how I ended up getting them, usually people that I knew or from networking with other people, yeah... a lot of my preceptors worked at clinics where I was already kind of familiar with their EMR...this made it easy for me.

P7: I did a lot of networking with my colleagues and I tried to call the people that were recommended by my school... I called a lot a lot of people and left a lot of messages, I would say with $90 \%$ of people did not call me back, I sent out a lot of e-mails with contacts that I was given, I also went into practices with my resume, I don't think that I actually got any preceptors that way. Most of them were colleagues, friends ...I worked with, NPs, or doctors.

Some participants reported maintaining the relationships with some of their preceptors after graduation; they contacted their former preceptors for information and advice after graduation. 
Participants also noted that though they were usually assigned one preceptor per site, other providers at the site were helpful in sharing some learning opportunities that are normally hard to come by.

P8: my preceptors, if there was something interesting, they would always want to pull me into that, or if there was even another provider had something interesting, they would always pull me into that, so that I could see things that you might not see every day...

The tendency to learn from all available providers as opposed to one preceptor continued into the post-graduation stage of transition when a novice NP often would reach out to any available provider as opposed to the collaborating physician only.

Table 13

Theme IV: Pre-graduation: Transitioning in Clinical Environment and Learning. Relationships and People

\begin{tabular}{llc}
\hline Subtheme/ & Participant's Comment & Summary \\
Code/ & & \\
Relationships & \\
and People & & \\
\hline
\end{tabular}

\begin{tabular}{lll}
\hline Relationships & P3: I sent out so many resume to ask for preceptor, no one will take & Rejection
\end{tabular}
and People me...

P3: See I work with this doctor and then the other doctor will say, have Team a suture, do you want to see. So this doctor will take me you know and precepting I will talk to my preceptor and this doctor said I can go with him to look at it. Yes go ahead! So they kind of all take care of me...

P4: I probably have every clinical there is ...names places and Familiarity locations...but a lot of the how I ended up getting them, usually people that I knew or from networking with other people, ye... a lot of my 
preceptors worked at clinics where I was already kind of familiar with their EMR...this made it easy for me.

P5: I was actually accepted for my 1st rotation in like an Indian Reservation. And someone agreed to take me on and that was probably 3 to 4 hours away from my house and then literally less than 2 weeks before the rotation started the MD called me and told me he said I am not going to be able to follow through with your clinical because there is MD student that needs a clinical here so I am sorry, good luck. And I was devastated...

P7: I did a lot of networking with my colleagues and I tried to call the people that were recommended by my school... I called a lot a lot of people and left a lot of messages, I would say with $90 \%$ of people did not call me back, I sent out a lot of e-mails with contacts that I was given, I also went into practices with my resume, I don't think that I actually got any preceptors that way. Most of them were colleagues, friends ...I worked with, NPs or doctors.

P8: So the school did not provide like a list of preceptors or? They had a list but then you were not allowed to contact anyone, it was very weird. ...it was so hard you pretty much take anyone you could get and there was no way of telling if they were going be good teacher... You basically had to cold call people but then you get in trouble for cold calling people am, I ended up finding couple of my preceptors by e mailing a list...

P8: my preceptors if there was something interesting, they would always want to pull me into that, or if there was even another provider had something interesting, they would always pull me into that so that I could see things that you might not see every day...

P9: ...I had to find everybody by myself and school did not provide
Rejection

Cold calling/

Familiarity

Rejection

Cold calling/

"No way of telling

if a good

Teacher"

Team

Precepting

Cold calling/

Rejection

Cold calling 
any help and I literally called tons of clinics until somebody says yes, it was very horrible.

P10: And my search strategy quite literally consisted of going to Rejection/ various offices within the geographic area, writing their phone number, Familiarity and then I spend the day cold calling...

P12: It was hard to find a clinical site... people were not as willing to Familiarity take students or they were already full, the school did not do a great job to find a lot of clinical sites, and for a few of them I used friends that were already working as NPs...

P12: I did a lot of clinical in acute care and UC and I was an ER nurse Rejection so I felt really comfortable there because I felt like that stuff was familiar.

P13: ...my 2nd preceptor dropped out...

P16: ...we had to find our own sites, to find own preceptors, which Cold calling/ fortunately I was working and where I had access to the whole Familiarity ...system directory so I just started sending out individual e-mails to family practice and pediatric providers to see if they could take would be interested in taking a student...

P16: ...my husband made 100s of phone calls for places ... lots of like Rejection/ calling and asking and begging... you feel like OMG, what is wrong Cold calling with me I can't get these clinical sites... I had my worst time with pediatrics, I had a physician lined up and he changed jobs and then he could not precept me...

Note. Theme IV. Relationships and People.

Post-graduation: Learning in clinical environment. When asked about the transition time after graduation and its support through the clinical environment, participants identified 
three major categories of people, relationships and communication, and space (see Table 14). Namely, participants described their experiences with their clinical environments, which included collaborating physicians, other providers, space arrangements, and various communication patterns.

People. Participants reported that they expected structured support when onboarding for their NP job after graduation. However, instead they experienced inconsistent learning experiences depending on their work environment.

Learning from everyone. All participants had a collaborating physician assigned to them; however, in most cases their collaborator was not the main sources of the information. Participants reported learning from those providers who were available to them on any given day. Participants reported that they often tried to ask questions from different providers to avoid being a burden, "P1: I try not to go to the same person all the time." They reported that it was helpful to learn various providers' styles, but not having one dedicated person to learn from created inconsistency (variable teaching styles and treatment methods) in learning the role during this role transition, "P11: ...the one problem that I had was that I found that this physician did not do things evidence-based." Additionally, it was difficult to approach people with questions since participants often were less familiar with "random" providers:

P6... so those days rotating I did not know the person well, that could be a little bit frustrating.

P12: It was a different doctor kind of every day, it was like at that time there were six of them, so it was just kind of whomever. 
P13...they all sort of took turns, I had a lot of different doctors I worked with, which was kind of positive and kind of negative but it meant that I did not feel I had a great good like mentoring.

In addition to learning from all providers, participants reported that they were learning together with medical residents, which was common if participants worked in an inpatient setting. Participants also noted that when working in inpatient environments, they often shared responsibilities for patient care with the attending physicians who frequently provided them with guidance. Though participants acknowledged that attending physicians were often helpful, they also stated that they were not as familiar with the NP role; thus, they treated participants more like medical residents; participants often felt alone and misunderstood in this position:

P13: ... I had kind of an unusual first job I think, it was in patient position but I was working on a team with medical residents and mostly medical residents... had a lot of attending physicians...I learned a lot of things sort of passively because I was working with residents and a lot of questions were answered for them, but my orientation was basically waiting for my credentialing...

Participants stated that collaborating with other NPs in their work environments helped them to learn their role. Participants reported that NPs were more familiar with their role and had more realistic expectations, which made it easier to approach NPs with questions.

P2: For the most part I just learned on the go from the other NPs. 
P8: Also there is four other advanced practice providers in the practice and I would say like three of them are really good, kind of like mentors to me and support when I am feeling ... not knowing what I am doing and that kind of thing.

P9...I learned a great deal from them, NPs there were on my level, and they would take the time to explain things to me...

Data showed that, both before and after graduation participants often reported having to learn from different providers. Frequent change of clinical sites and preceptors prior the graduation followed by learning from those providers who were on site during the participants' transition to practice. While frequent change in preceptors allowed participants to become familiar with different styles and settings, it was also reported to create inconsistency in participants' learning of their role. Additionally, participants reported variable access and opportunities to learn from their collaborators.

Collaborator on paper. Participants reported that often their assigned collaborators were "collaborators on paper." They noted that their collaborating MD was often busy and had own busy schedules or additional jobs and responsibilities including medical students and off site jobs; participants reported that collaborators had no "carved out time" to train novice NPs. P6... and then you don't want to feel like you are holding that person down because they have to see patients too, so you did not want to place extra burden on them to ask some questions and disturb their day.

P7: I also consulted the docs that were in this clinic... but it is hard to always be asking them questions when they are busy too... 
P8: ...I did not have like...specific person that I was ever like would precept me or that had extra time to help me so like ...they might say like or you have question today ask Dr....but she would not have any time carved to help me so it would be really hard to get support because she still had a full schedule and then she is supposed to help me with my patients too, especially that first like month or two because I was ... learning curve as ... it is not like quick questions...

P9: ... if I had a question, there are other doctors that I can converse with yes, but the specific collaborator on paper, no I don't really deal with him.

P14: ... she had some scheduling conflicts because she was doing a lot, she was working two-three jobs and full time, she also was running this practice.

P14: ...post-graduation I had collaborators on paper and... I did not have anyone to really break down my day to... just come home and vent to my husband like I wish I may be had some support...

P16: Like she was that sick and the doctor like no no just look up, just google it and figure what to do and I was like OMG please don't let this woman die...

Overall, participants reported that their collaborating MDs were rarely the only source of information for them; rather, they learned from all providers available to them. Collaborators' busy schedules and lack of familiarity with NP role were reported to often prevent them from providing NPs with needed assistance. Not having consistency in their preceptorship, participants used other resources to self-educate. 
Table 14

Theme IV: Post-graduation: Transitioning in Clinical Environment and Learning. People.

\begin{tabular}{|c|c|c|}
\hline $\begin{array}{l}\text { Subtheme/ } \\
\text { People/Collabo } \\
\text { rators and } \\
\text { Preceptors }\end{array}$ & Participant's Comment & Summary \\
\hline \multirow[t]{6}{*}{$\begin{array}{l}\text { Learning from } \\
\text { Everyone/ } \\
\text { Collaborator on } \\
\text { paper }\end{array}$} & $\begin{array}{l}\text { P1: I try not to go to the same person all the time, there is } 2 \text { NPs with me } \\
\text { in peds and there is one pediatrician, so sometimes I will go to } 1 \text { NP and } \\
\text { sometimes I will go to the other and then sometimes I will get pediatrician } \\
\text { who is usually the most busy... so we all kind of work together... I work } \\
\text { at the....center, so there is a bunch of providers, bunch of departments, } \\
\text { there is a lot of people there. }\end{array}$ & $\begin{array}{l}\text { Learning from } \\
\text { Everyone }\end{array}$ \\
\hline & $\begin{array}{l}\text { P2: ...there were the } 2 \text { other NPs I worked with, they taught me some, the } \\
\text { surgeons when they were in ...may be, but for the most part I just learned } \\
\text { on the go from the other NPs... }\end{array}$ & $\begin{array}{l}\text { Learning from } \\
\text { NPs }\end{array}$ \\
\hline & $\begin{array}{l}\text { P2: My collaborator, like my collaborating physician? O no, he did not } \\
\text { know how to do half the stuff, like the discharging stuff or anything. }\end{array}$ & $\begin{array}{l}\text { Collaborator } \\
\text { on paper }\end{array}$ \\
\hline & P3: ...I think right now there are probably 8 or 10 doctors... & $\begin{array}{l}\text { Many } \\
\text { collaborators }\end{array}$ \\
\hline & $\begin{array}{l}\text { P6: ... so I had a collaborating physician but she is not on site, so I was } \\
\text { able to have... when I first started they did like orientation where....I } \\
\text { floated ... to } 2 \text { other sites and worked alongside different providers, it } \\
\text { could have been MD, NP, or PA, so I kind of shadowed their work, right } \\
\text { before I started at my site on } 2 \text { occasions... }\end{array}$ & $\begin{array}{l}\text { Learning from } \\
\text { Everyone }\end{array}$ \\
\hline & $\begin{array}{l}\text { P6:...there was some frustration at times because just depending on like } \\
\text { you know when I started I was not every day I would have the same }\end{array}$ & $\begin{array}{l}\text { Burden to a } \\
\text { busy }\end{array}$ \\
\hline
\end{tabular}


not know the person well, that could be a little bit frustrating and then you

don't want to feel like you are holding that person down because they have to see patients too so you did not want to place extra burden on them to ask some questions and disturb their day.

P7: I had I actually kind of reached back to some of my preceptors that I had before...I went back to her and kind of asked her what she would recommend in terms of resources to use in my clinical practice .... I also consulted the docs that were in this clinic... but it is hard to always be asking them questions when they are busy too...

P7: ...he is pretty good at guiding me but he is not really teaching me that much...

P8: ...I think I have more than one mentor, some of the other NPs that work there kind of look as mentors, they are basically in the same position that I am in, so he is definitely is not like my only mentor, also there is 4 other advanced practice providers in the practice and I would say like 3 of them are really good, kind of like mentors to me and support when I am feeling ... not knowing what I am doing and that kind of thing.

P8: ...I did not have like...specific person that I was ever like would precepting me or that had extra time to help me so like ...they might say like or you have question today ask Dr....but she would not have any time curved to help me so it would be really hard to get support because she still had a full schedule and then she is supposed to help me with my patients too, especially that 1 st like month or 2 because I was ... learning curve as ... it is not like quick questions...

P9: ... if I had a question, there are other doctors that I can converse with yes, but the specific collaborator on paper, no I don't really deal with him. I learned a lot from the MDs, but their knowledge base so vast that sometimes it is kind of hard for them to actually really teach you, I
Learning from less known providers

Burden to a busy preceptor

Collaborator on paper

Learning from NPs

Burden to a busy preceptor

Collaborator on paper/ Learning from NPs 
learned a great deal from them, NPs there were on my level, they would take the time to explain things to me and very helpful.

P9: ... MDs? They were helpful too but they were so busy

Collaborator and they did not have a whole lot of time to teach me things, like NPs on paper did... got somebody on paper ye, but I don't really talk to them...

P10: ... whoever was on that day... if I am not if I can't find the answer Learning from within I would say within 5 minutes, that I am reasonably confident with, Everyone then I will try to run and ask the attending physician is there that day or another NP with some experience and just grab some ideas off of them...

P11: ...the one problem that I had was that I found that this physician did Different style not do things evidence based, so she you know would treat things that you know when I am looking and studying and I am like this is not really the way most people are treating things now...

P12: ...they had me just to following the physicians around, I spent most Learning from of my time with the physicians, at this job, although there were like 4 NPs Everyone in the office... they alternating them when they are in the office and they are doing like endoscopy and stuff so really it was a different doctor kind of every day, it was like at that time there were 6 of them, so it was just kind of whoever..

P13: ...I work with 8 different cardiologists... my cardiologists has their Learning from own clinic schedule and generally I just if I have a question I page them...

multiple collaborators

P13: ...I had kind of an unusual first job I think, it was in patient position Learning from but I was working on a team with medical residents and mostly medical residents and residents... had a lot of attending physicians...... because they all sort of attending took turns I had a lot of different doctors I worked with, which was kind of physicians positive and kind of negative but it meant that I did not feel I had a great 
good like mentoring, I mean I learned a lot of things sort of passively because I was working with residents and a lot of questions were answered for them but my orientation was basically waiting for my credentialing...

P14: ...I listen and everyone is different so it is nothing consistent Inconsistent message so at times I just google it...

Learning

P14: ...NP is supposed to practice independently but they also understand Learning from that like for me coming in a ER I am coming and never been in ER so ask residents and them a lot of questions, so whoever is the attending, that day, there is attending always 2-3 attendings, at any given time, so I just pick one and I body up physicians with that person.

P14: ... she had some scheduling conflicts because she was doing a lot, she Burden to a was working 2-3 jobs and full time, she also was running this practice... busy preceptor she brought me in so just kind help her transition but with that did not express until after the fact until she was ready to let me go... sometimes she come in once a week, she may respond to my questions...

P14: ...post-graduation I had collaborators on paper and... I did not have Collaborator anyone to really break down my day to... just come home and vent to my on paper husband like I wish I may be had some support...

P14: ...I come to realize she just ignored my texts. And she, there were Collaborator times there were patients I really thought she needed to see or discuss with on paper me, I would have the front desk call her in hopes may be at some point she would want to touch base with them and that way it will catch me or wise versa and some days I was .. it was and there really was not any consistency as to when or why she chose to calling...

P15: ...I can always text him, or I can go ask one of the other providers in Learning from the clinic... attending because they have residents they have to work with, Everyone they are actually going through a process and some of them did not 
actually care to have an NP cause you are supposed to be independent so why am I helping you...

P16: If I had been in the more like supportive environment with the better Collaborator orientation, I likely would have felt less stressed but I was so worried on paper about OMG what if I harm somebody and what labs do I order ... like she was that sick and the doctor like no no just look up, just google it and figure what to do and I was like OMG please don't let this woman die...

Note. Theme IV: People. Collaborators and Preceptors. Learning from Everyone. Collaborator on paper.

Relationships and communication. When describing relationship and communication experiences, participants commented on their relationships with the collaborating MDs, other NPs, residents and attending MDs, friend NPs and former NP preceptors, referring physicians, staff, and management (see Table 15).

Participants also reported that their managers were either their collaborating MDs or nonmedical staff. Participants reported that in most cases their employers were less familiar with NP role and expected them to start functioning independently in their new role or to "hit the floor running."

P1: The organizations, because they felt that because I am an FNP, I should be able to do those things, they are not clinical, so they do not really understand, they think that if you got FNP behind your name you should be able ... They think once we finish school we should be able to hit the floor running.

P2...you kind of just get thrown and figure it out P5: I worked there for about a month but that MD had never worked with NP before, so he really did not know who was whose role... 
P14: ...I felt like I was sink or swim, good luck.

As a result, participants would often find themselves in a "sink or swim" situation while transitioning to their new responsibilities. There was one instance; however, when one employer expected to train a new NP "from the ground up." Thus, participants reported that their employers' expectations were inconsistent and unrealistic. Though in most cases employers would gradually increase the number of patients on NPs' schedules, they often anticipated novice NPs to have enough knowledge and clinical experience to see high volume of complex patients relatively quickly after the start date.

Further, participants reported difficulties with learning from their collaborating MDs. More specifically, they noted that though their collaborators respected their clinical decisions, NPs' communication with their collaborating MDs was often limited either due to MDs being off site or being busy with own patient panel or medical residents: "P11: I kind of felt I taught myself a lot ...but we never even had like a supervising MD contract..." Participants reached out to their collaborating MDs when necessary but these communications were often limited due to the participants' fear of being a burden to their collaborator. On occasion participants reported scheduling some time with their collaborator to discuss specific questions: "P6: So I was there a little bit of time one day a week for may be 30 minutes ...we both had time allocated to do that, questions about lab work." Occasionally when access to their collaborating MDs was limited, participants noted having to reach out to their NP friends or their formal preceptors to help with the transition. Additionally, when communicating with referring providers, Participants reported 
feeling intimidated; however, participants reported increased comfort level as they practiced longer.

Further, those participants who reported working in inpatient clinical environments repeatedly reported having to share patient care opportunities with the attending MDs and medical residents. Participants reported that attendings and residents often lacked knowledge about the NP role, which made their work uncomfortable as they were often treated like medical residents: "P2: they threw me with the bunch of med students and started asking questions about these scans like I did not know the answers to..."

Participants reported having positive experiences when other NPs who were working alongside with them. More specifically, they noted that NPs were much more familiar with their role and their baseline knowledge; thus, they felt more comfortable to "bounce ideas off of them."

P12: ...I worked in an office that shared with four other NPs, so literally in the same space with me ... so I could always just say hey what do you think about this...

P13: In the clinic I have two other NPs ... they are around and we can bounce ideas off of each other, which is nice...

Finally, participants reported having positive transition experiences when people in their work environments were friendly and supportive. For example, experiences were reported as positive when participants were supported by an individually assigned staff member to help novice NP with managing EMR, schedules, and patient follow up. 
Theme IV: Post-graduation: Transitioning in Clinical Environment and Learning. Relationships and Communication

\begin{tabular}{llc}
\hline Relationships & Participant's Comment & Summary \\
and & & \\
Communication & &
\end{tabular}

\begin{tabular}{ll}
\hline Relationships & P1: The organizations, because they felt that because I am an FNP, I \\
and & should be able to do those things, they are not clinical, so they do not \\
Communication & $\begin{array}{l}\text { really understand, they think that if you got FNP behind your name } \\
\text { you should be able things that we do here. They think once we finish }\end{array}$ \\
& school we should be able to hit the floor running.
\end{tabular}

P2...you kind of just get thrown and figure it out, I figure there is

"You get probably NP jobs when there is something much more formalized when they are onboarding a lot of NPs...

P2: they threw me with the bunch of med students and started asking questions about these scans like I did not know the answers to...

Expected to "hit the floor running."

Y thrown into." Treated like
residents

P4: ... for me the most overwhelming part is having to, consult with the referring provider, I think sometimes it can be difficult, you know when the patient has multiple doctors...

P5: I worked there for about a month but that MD had never worked Unrealistic with NP before so he really did not know who was whose role...

Intimidated by referring providers

expectations

P6: Well, the expectation was that I already been trained so already "right out of the been through school so my job is supposed to ... patients, assess, $\mathrm{dx}$, gate" come up with tx plan for all my patients, so there really was not a whole lot of time for me ... right out of the gate...

P6: So I was there a little bit of time one day a week for may be 30 Had Time with minutes ...we both had time allocated to do that, questions about lab the collaborator work, that was difficult as a new provider... 
P6: I was lucky to be at the site that welcomed me...

Welcoming site

P7: I had I actually kind of reached back to some of my preceptors that

Reaching out to I had before

former

preceptors

P11: ... he expected me to hit the ground running for sure. He was not

Expected to "hit going to give me much orientation at all.

the floor

running."

P11: I kind of felt I taught myself a lot ... but we never even had like a No Time with supervising MD contract...

the collaborator

P12: ... once I was done with the week or 2 of the orientation, you are by yourself...

Two weeks and

then by yourself

P12: ...I am used to get thrown into the wall you know and I worked

NP to NP

in an office that shared with 4 other NPs so literally in the same space with me, so I could always you know and they have been there a while, so I could always just say hey what do you think about this...

P12: The only thing was a little bit hard when I started they did not No support staff give me my own nurse at first...

P13: I think their expectations for me for my 1st job were very low, so I think they figured they were going to train me from the ground up...

Low

expectations

P13: the resident physicians are learning too, so they don't necessarily Learning with know how to interact with the different staff anyway, so I mean they residents rotated monthly so some months I had a very nice month and some months I just really want to really not want be there.

P13: In the clinic I have 2 other NPs ... they are around and we can NP to NP 
bounce ideas off of each other, which is nice...

P14: it was a different experience being trained by NPs and then going out working with not many NPs and realizing that what you are doing and what you are coming in with, your expectations and their expectations are completely different.

P14: ...I felt like I was sink or swim, good luck, and that transition into trying to be a provider, wearing that hat, diagnosing, prescribing, all of those things with the kind of support I had working with NPs, being trained by NPs and coming out with MDs, it was sink or swim, you are supposed to be independent, so you got this...

P14: I kind of had to choose learn on my own ...I...I literally was texting my friends, I have this patient, looks like this, I think this this this, what do you think...

P15: ...when we had our meeting he was saying you know some of my deficiencies for physical exams and those kind of things and I said you know I asked you for help in those areas and you taught me nothing you know, I had to teach myself with u tube and you can't fault me for not knowing what you wanted me to know when you gave me no instruction...

P15: ...I was just standing observing his surgery and he had a resident with him so the resident was always up near the OR people so I was usually standing off back in the background so I did not really see and he was always interacting with the resident and rest of the OR staff and anesthesia so I really did not learn anything from going into the OR with him...

P15: I pulled him aside while he was with the resident said hey, could you go over those provocative testing and it was like 10 minutes in
Working with

MDs/lack of

role

understanding

"Sink or swim."

Texting friends asking for help

Lack of teaching from MD

Lack of teaching from MD

Lack of teaching from 
exam room and he... shoulder, knee, elbows, hips, you know in about $\mathrm{MD}$

10 minutes while I was trying to jag down everything he was saying

the best I could and so I basically taught myself provocative testing by

watching u tube...

P15: we just we seat next to each other... and I can always text him, or

Supportive

I can go ask one of the other providers in the clinic...

collaborator

Note. Theme IV: Relationships and Communication. Employer's expectations. MD NP relationships.

Space and proximity to other professionals. Participants reported that physical proximity

to other providers significantly facilitated knowledge transfer during their role transition. Codes of never alone and learning alone emerged from the data (see Table 16). Participants reported that having other NPs as well as other professionals in close proximity allowed them to ask questions and feel supported in their learning efforts.

Never alone. More specifically, having other providers in the same office space made it easier for the novice NPs to ask for clarifications and share the ideas related to patient care.

P2: Literally my desk was right next to two other NPs or the fellow or residents, if I did not know, I just asked... ...there are always people around... there were very few times that I was ever physically alone as an NP...

Learning alone. However, being the only NP in practice presented additional challenges of experiencing learning the role in isolation. Some NPs reported to be the only provider in the office with the "ghost collaborator" who were mostly absent, "P14: I did not have her presence, did not have support that she said I was going to get, so I kind of had to choose learn on my own...." Being the only provider and handling full patient schedule right after graduation was 
reported as being especially stressful and difficult experience.

Participants reported that it was harder to transition when they were the first or the only NP in practice. They reported that when they were the only NP in the practice, their role was less understood by management and collaborators. Frequently the employers' expectations were reported as less realistic as well as less support was provided to the novice NPs, "P15: I was the first NP to be hired to work along with one of the surgeons, and so they did not have any orientation organized for me."

Familiarity. Another aspect reported by participants was related to being familiar with the clinical site or providers prior the start date of their jobs. More specifically, previous experiences working at the sites or working with the site providers were reported having a positive impact on participants' communication, thus, facilitating knowledge transfer during their onboarding process and role transition, "P1: In my pediatric rotation I was actually precepted at the job that I currently work at now, so that was really helpful."

Own space. Additionally, some providers reported feeling more welcome when they were assigned a dedicated space or a desk, "P4: As a provider I was given that whole office space almost like I had my own office...it was ready to have me so that felt good.” Some participants reported having no assigned space including the space to place their belongings when reporting to their jobs, "P15: I was kind of hovering on the corner of someone's desk... I come here and you don't have a spot for me..." One respondent reported having to purchase own computer and equipment. Having dedicated physical space along with working equipment was noted to be an important part of participants' role learning transition experiences. 
In summary, data showed that having other professionals in close proximity was helpful in participants' learning their new role; however, not having other advanced providers close by made transition more difficult. Prior the graduation, participants reported never being alone taking care of their patients. More specifically, they reported being "attached to the hip" of their preceptors. The transition from complete dependency on another person for making patient care decisions in a clinical learning environment to a complete autonomy, combined with working alone in some cases, was one of this study findings. Further, being familiar with the space and providers prior hiring helped some participants feel more comfortable in a new role. Namely, being surrounded by familiar things and people was reported as helpful, both before and after graduation. Because having an assigned preceptor was rare, it was important for novice NPs to be surrounded by their colleagues-providers to continue learning their new skills.

Table 16

Theme IV: Post-graduation: Transitioning in clinical environment and learning. Space. Proximity to other professionals.. Learning Alone. Never Alone. Familiarity Own Space.

\begin{tabular}{|c|c|c|}
\hline $\begin{array}{l}\text { Proximity to } \\
\text { other } \\
\text { professionals }\end{array}$ & Participant's Comment & Summary \\
\hline \multirow{3}{*}{$\begin{array}{l}\text { Proximity to } \\
\text { other } \\
\text { professionals }\end{array}$} & $\begin{array}{l}\text { P16: ... in my previous job I had to buy my own computer and I } \\
\text { was told to practice on the weekend... }\end{array}$ & Equipment \\
\hline & $\begin{array}{l}\text { P1: In my pediatric rotation I was actually precepted at the job that } \\
\text { I currently work at now, so that was really helpful. }\end{array}$ & Familiarity \\
\hline & $\begin{array}{l}\text { P2:Literally my desk was right next to } 2 \text { other NPs or the fellow or } \\
\text { residents, if I did not know I just asked... ...there are always }\end{array}$ & Never alone \\
\hline
\end{tabular}


people around... there were very few times that I was ever

physically alone as an NP...

P3: ... we are partners at night, so it is just two of us... work very

Never alone

closely... working in the hospital I always got resources, there is

so many NPs, doctors, professions...

P3: I work with the doctor I have known for a long time... we

Familiarity already had pretty good relationship before I started working for him and then we kind of just not changed the role... I work in the hospital; I have been the nurse there for 10 years, so I know where stuff is...

P4: As a provider I was given that whole office space almost like I

Own Space had my own office... it was ready to have me so that felt good.

P6: ...on site, I always had another provider there with me... Never alone

P8: ...some of the other NPs that work there kind of look as Learning from other mentors, they are basically in the same position that I am... you NPs don't have to feel embarrassed asking them questions because you know they feel that they were kind of treated like I was in their position once...

P12: ...you do have a collaborating physician obviously although I "Ghost" collaborator never met him, I have never even talked to him on the phone so that relationship was kind of weird because you don't feel comfortable calling him...

P14: I was the 1st NP in ED, they had experiences in NPs in other The only NP parts of the hospital but I was the 1st on in ED.

P14: I did not have her presence, did not have support that she said "Ghost" 
I was going to get, so I kind of had to choose learn on my own... collaborator/Learning Alone

P15: I was the 1st NP to be hired to work along with one of the The first NP surgeons and so they did not have any orientation organized for me.

P15: I was kind of hovering on the corner of someone's desk... I No space come here and you don't have a spot for me...

Note. Note. Theme IV: Proximity to other professionals. Learning Alone. Never Alone. Familiarity. Own Space.

\section{Theme V: Transitioning to a Greater Autonomy and new Responsibilities}

In the fifth interview question participants were asked about their experiences with autonomy and new role responsibilities during their transition to their new NP role both during them attending NP program and after graduation. Participants' degree of independence and their experiences with diagnosing and prescribing in their new role were investigated.

\section{Pre-graduation: Transitioning to a greater autonomy and new responsibilities.}

When asked about their transition to a greater autonomy and new responsibilities prior the graduation, participants identified two major categories of students' autonomy and preceptors, and critical thinking (see Table17). Participants' learning how to practice their new skills autonomously was affected by their preceptors' comfort level with the student and their preferences for student's autonomy level, preceptors' use of evidence in their practice, and patient care opportunities that allowed students to develop the differential diagnosis skill.

Students' autonomy and preceptors. Participants revealed that preceptors' trust and comfort level with the student often guided preceptor's decision regarding the degree of 
student's autonomy during their clinical experiences. Participants reported that as they had new preceptors with each rotation, it would take weeks to months for them to advance to when they could practice somewhat autonomously.

P11: ... if you think how much time you actually spend in clinical, you get about one day a week for like a semester it is like half a semester, you go like for 10 times total and I spent like 4 of those times shadowing. So that really start to feed in to like how much you are actually able to do and learn.

As a result, shorter active learning times were not as conducive to students' learning since a portion of those experiences consisted of shadowing the providers.

P7: ...I think it was like I would say two to three weeks and it was more of a comfort on her side than mine end. I think that she wanted to feel me out and make sure I was confident taking care of patients.

P12: It kind of depended on the preceptor, I mean may be like a couple of weeks for family practice shadowing ... but then I did work with the pediatrician who never wanted, would want to go in with me with everyone, like did not want me to go in by myself, so it kind of depended on who you...

At times though preceptors were too busy to allow for student's autonomous practice:

P15: ... it be like a couple of weeks into the clinical setting before that comfort level would kind of develop to where they would realize that they would be comfortable, and their comfort level with me to trust that my assessment were appropriate.

However, when the preceptor was comfortable with the student, it helped participants build 
necessary skills and self-confidence in performing in their new role, P7: ... she really trusted me and allowed me to just do everything which upped me my confidence and allowed me to feel confident with doing my role as a NP on my own... Besides building preceptors' trust and length of the clinical experiences, preceptors' preferences affected the ability of participants to practice semi-independently. Most participants reported at least one clinical rotation in which their preceptor allowed only shadowing experience removing the hands on portion of the experience and offering students no autonomous experiences. In some instances participants reported these inadequate clinical experiences to their faculty and attempts were made to correct these to provide students with more appropriate practicum. Participants noted that their shadowing time was less when with NP preceptors as opposed to MD preceptors.

P8: I would say like the NP preceptors were more like understanding of my process and what I would be expected to know, and I feel like sometimes the physician preceptors were more like observation a little bit, and they just kind of were not always I feel like it was not as interactive of an experience...

P12: I feel like NPs like been a little bit more comfortable with me being more independent than the physicians did.

Participants reported that shadowing experiences did not adequately allow them to learn their new role.

Additionally, participants reported that during their attempts to learn new NP skills at their clinical sites, their RN skills were occasionally used by their preceptors placing them in a 
more task-oriented role, "P1: I kind of felt like we were his assistants at his job so..." More specifically, participants reported starting IVs, taking vital signs, and other measurements on their patients performing medical assistants' duties.

P5: I feel like some of the times they just kind of looked at having a student as more of ...an extra assistance who can you know run a set of vital signs for me or ...you are treated most at the level of a medical assistant instead of an NP or a provider level... P7: I think they appreciated me being there but I also helped with things outside of my role as a NP so they would ask me to like draw labs or take BP on people, or help that was like procedures, I think they kind of used me as extra hand... learning how stay in my role as NP and not deviate and do things that are MA and nursing staff should be doing.

P16: I did not get a lot of teaching from her; I was used more like an extra set of hands because she was so swamped.

Participants stated that they did not mind helping busy preceptors, though they realized that their clinical hours experiences were limited.

In addition to occasional preceptors confusion the roles of an $\mathrm{RN}$ and NP, the ultimate patient care responsibility was always with the preceptor, " $\mathrm{P} 4 \ldots$ in school you knew that there is somebody who will come behind you. When you actually out there other than your collaborating physician, I mean it is pretty much just you..." Participants reported that they did not have many worries during their clinicals at school since their preceptor was the one responsible for the care 
outcomes at all times. Participants stated that if they did not know how to treat patient, their preceptors would take over.

P12 : I always had a preceptor to fall back on, that really made me comfortable, you know you are not the one totally responsible for this patient, so I don't know may be I just did not take it seriously or I did not know I did not care as much I don't know, because you are a student, you are really kind of working with someone else so the responsibility falls on them ...it did not seem as daunting as a new grad when it is your responsibility...it did not really hit me until my first job I would say.

Participants also reported that most of the times they did not have an opportunity to follow up on their patients since their clinicals were scheduled very sparingly, often once or twice a week, which led to increased reliance on their preceptor. Overall, data showed that participants' autonomy was impacted by preceptors' comfort level with NP students and preceptors' workload.

Critical thinking. Besides not carrying patient care responsibility that would approximate the reality of clinical practice, participants reported having insufficient experiences with practicing making differential diagnoses. Because many patient care opportunities were reported as either simple, or patients had already been diagnosed (e.g., in specialty care), participants reported not having enough opportunities to practice critical thinking skill of a prescriber. P13: ...most of them I think I already knew what the diagnoses were though, or the diagnoses were not difficult... 
P14: ... unfortunately, I think that is where I feel there be a little I felt not as prepared, there was no diagnosing for me during my rotation. .. lot of patients that would come in, they already knew why they were coming, especially since I was working in specialty settings...

In addition to limited patient care opportunities, participants reported occasional confusion caused by their preceptors' use of evidence.

P5: ...during my last rotation I had mentioned I was working with the MD who was probably retiring and I don't think that the current guidelines were number one thing on his mind.

Because participants were taught to always use evidence when treating patients, any preceptors' deviation from the guidelines was viewed as inconsistency, which caused confusion and prompted participants to pull back from practicing semi-independently.

In summary, participants' autonomy in patient care during their clinical rotations was often limited due to the frequent lack of preceptors' trust and comfort level with the NP students who they had for a relatively short clinical rotation. Additionally, preceptors' workload, which at times did not allow for the teaching time, often prevented students' autonomous practice during their clinical experiences. Role confusion when used as RNs during their NP rotations and dependence on their preceptors for most aspects of patient care, including differential diagnosis skill, was frequently reported. Though most reported having some of their rotations as more successful and independent, it was often not sufficient for the participants to obtain the necessary 
skills. This lack of autonomy while attending NP schools was changed to a complete autonomy as participants graduated and advanced to NP practice.

Table 17

Theme V: Pre-graduation: Transitioning to a Greater Autonomy and new Responsibilities. Students' Autonomy and Preceptors.

\begin{tabular}{ll}
\hline Students' & Participant's Comment \\
Autonomy & \\
and \\
Preceptors
\end{tabular}

P1: I kind of felt like we were his assistants at his job so... he expected us to

Extra set of do part of the exam...

hands

$\mathrm{P} 4 \ldots$ in school you knew that there is somebody who will come behind you.

Responsibility is

When you actually out there other than your collaborating physician, I mean on Preceptor it is pretty much just you...

P5: I feel like some of the times they just kind of looked at having a student

Extra set of as more of ... an extra assistance who can you know run a set of vital signs hands for me or ...you are treated most at the level of a medical assistant instead of an NP or a provider level...

P7: ... she really trusted me and allowed me to just do everything which Preceptor's Trust upped me my confidence and allowed me to feel confident with doing my role as a NP on my own...

P7: I think they appreciated me being there but I also helped with things

Extra set of 
outside of my role as a NP so they would ask me to like draw labs or take hands

BP on people, or help that was like procedures, I think they kind of used me as extra hand... learning how stay in my role as NP and not deviate and do things that are MA and nursing staff should be doing.

P7: ...I think it was like I would say two to three weeks and it was more of a Preceptor's Trust comfort on her side than mine end. I think that she wanted to feel me out and make sure I was confident taking care of patients.

P8: I would say like the NP preceptors were more like understanding of my Had more of an process and what I would be expected to know and I feel like sometimes the physician preceptors were more like observation a little bit, and they just interactive kind of were not always I feel like it was not as interactive of an experience with experience...

NPs as they were more familiar with the role

P11: ... if you think how much time you actually spend in clinical, you get Shadowing is about one day a week for like a semester it is like half a semester, you go not as effective like for 10 times total and I spent like 4 of those times shadowing. So that really start to feed in to like how much you are actually able to do and learn.

P12: It kind of depended on the preceptor, I mean may be like a couple of More autonomy weeks for family practice shadowing or I am thinking that is about right but with NP then I did work with the pediatrician who never wanted, would want to go in preceptors with me with everyone, like did not want me to go in by myself, so it kind of depended on who you ... some of them were NPs, so I don't know, I feel like NPs like been a little bit more comfortable with me being more independent than the physicians did.

P12: I always had a preceptor to fall back on, that really made me Responsibility is comfortable, you know you are not the one totally responsible for this on preceptor, patient so I don't know may be I just did not take it seriously or I did not "not as 
know I did not care as much I don't know, because you are a student, you daunting." are really kind of working with someone else so the responsibility falls on them ...it did not seem as daunting as a new grad when it is your responsibility...it did not really hit me until my first job I would say

P13... in school I just asked what my preceptor would do... Responsibility is on preceptor

P14: ....I never had a situation that I had to do it on my own in any of my Responsibility is rotations. on preceptor

P15: ... it be like a couple of weeks into the clinical setting before that Preceptor's Trust comfort level would kind of develop to where they would realize that they would be comfortable, and their comfort level with me to trust that my assessment were appropriate.

P16: ...once you were with somebody and they kind of figure out ok, she I Preceptor's Trust can trust her on patients, you got more and more, as they trust your judgment better and they allow you do more... except for the places they were absolutely swamped and you were postponed at the same time...

P16: ...it was kind of a dual effort like they would let you do the work but Responsibility is then like put their opinion from experience like how they would do it on preceptor slightly different.

P16: I did not get a lot of teaching from her, I was used more like an extra Extra set of set of hands because she was so swamped.

hands 
Critical P5: ...during my last rotation I had mentioned I was working with the MD

Confused

Thinking who was probably retiring and I don't think that the current guidelines were number one thing on his mind... the guidelines the UpToDate it says you know we should be using macrolide, and he is just mm no this is my go to for this, so ...I just kind of had to let him do his thing, it was not my decision to prescribe the thing but that is what he did.

P11: ...one of them was not very good because the preceptor also had Confused questionable like clinical guidelines she was following or lack thereof...

P13: ...most of them I think I already knew what the diagnoses were Differential though, or the dx were not difficult...

Diagnosis

P14: ...unfortunately I think that is where I feel there be a little I felt not as Differential prepared, there was no diagnosing for me during my rotation. .. lot of Diagnosis patients that would come in they already knew why they were coming especially since I was working in specialties settings...

P16: ...in school, differential diagnoses, I think like, that is the biggest thing Differential that I think kind of sucks as our NP school, it is not medical school, so we Diagnosis don't learn about absolutely everything it could possibly happen...we did not get a lot of teaching in school about labs and I like, that was, I felt like I really struggled with that at first...

Note. Theme V. Students' Autonomy and Preceptors. Critical Thinking.

Post-graduation: Transitioning to a greater autonomy and new responsibilities.

When asked about the transition to a greater autonomy and new responsibilities during time after graduation, participants identified three major subthemes including wide scope, autonomy of decision making, and administrative duties (see Table 18).

Wide Scope. Participants reported transition to primary care and specialty care as daunting, poorly supported, and very challenging. Patient care wide scope combined with lack of 
support and lack of clinical exposure when in school, were noted to be the reasons why participants felt overwhelmed. Those who transitioned to specialty care reported having superficial knowledge base initially when caring for complicated clients.

P1...I feel like I am comfortable but there are still a lot of things that I see sometimes and I have never seen them before...

P5... even to this day when I see people come in with different stuff I just like oh you know it is you are learning every single day. I still sometimes wonder what the ... I am doing in this field, so it is not easy, take one day at a time...... such a huge learning curve, and the moment you are comfortable with something, something else comes up.

P14: ...very challenging lots of frustration, lots of thinking I chose the wrong profession....

Many questioned their decision to become an NP as their new responsibilities were overwhelming causing a lot of anxiety and fear of making an error. Some participants practiced in specialties and some planned to practice in specialty areas in the future. Many stated that focusing on a single specialty offers an opportunity to deepen one's knowledge about a narrower aspect of patient care as opposed to focusing on many elements of care included in primary care and having superficial knowledge, which is unsafe and uncomfortable. However, the initial period of transitioning to specialty care was shockingly hard due to the lack of knowledge.

Autonomy in decision making. Participants reported a sudden increase in autonomy after their credentialing process completion at work. Sudden change from depending on the preceptor to a complete autonomy was rather unexpected by the participants. 
P7: ... lot of autonomy since the beginning, which is nice but also a little scary, not knowing anything brand new NP seeing patients on your first day on your own is pretty intense...

P11: So in school there was no independent diagnoses or prescribing, I was writing by everything by my preceptor when I was in school, and then in practice you know, all of the sudden, I am handed the license and I can do it...

Prior the credentialing process most participants reported shadowing multiple providers at their practices; at that time, most of them did not have an opportunity to routinely make patient care decisions to help them learn their role. Some participants did not have any opportunities for training, including shadowing. Having a complete and abrupt autonomy of patient care decision making without adequate support was reported as stressful.

P14: Prescribing was ... on the go, so there was no... which has been one of the toughest things come in after graduation and having to do, especially working in the PC settings... Participants reported having to perform new skills of diagnosing, prescribing, and delegating. Some participants also reported that delegating to their staff was a new skill for them; changing from being an equal to giving orders was reported as required some adjustment.

P10: I guess it was ok to tell them to go do it, rather than do it myself. And that part has been a bit of a challenge in learning more delegation I would say.

P15: ...I have trouble delegating just because I don't want to sound bossy... Shared Decision Making. Participants who worked in inpatient environments reported sharing their patient care responsibilities with their attending MDs. Sharing responsibilities was 
reported as being helpful in some cases, and as times it was reported as impeding NPs practice at their full role.

P2: ...give the report to the fellow or the attending tell them about the kid....So I would go and like see the kid and write the note, they would put in a short note that they had seen the kid also, cause they were still responsible for them as well, but and the attendings were at some point...

Scope limitations. Participants also reported some scope limitations, which varied depending on their work setting. Most participants reported not being able to do procedures unless trained on the job as well as some limitations with seeing new patients. Lack of procedure training when in school translated into an inability to practice to NPs' full scope. Such limitation caused participants to be more dependent on other professionals for training and patient care.

P1: I had a patient that came in, she fell and hit her head and she had this huge gash on her forehead, and I knew right away that she needed sutures, so I had to send her to the ER or doctors care so that to suture, had I known how to suture, I would have been able to do it...

P13: I think they prefer if I don't see new patients.

One participant reported that her collaborating MD requested her to mimic her ways of practicing medicine; participant reported that this limited her autonomy of practicing her role. Lack of MDs' familiarity with NPs' role and scope led to inappropriate role modeling, limiting novice NPs' autonomy. 
P16: ... she told me when I was hired that she wanted to create, she wanted me to be just like her. And wanted me to practice just like her and write notes just like her, so it was about learning to do it exactly like her...

Administrative duties. Some participants reported having to perform assistive or administrative duties; these often were connected to practicing in an inpatient environment. Occasionally participants, who were practicing in an outpatient environment, reported performing clinic administrative and teaching duties. Participants stated that they preferred NP role duties to administrative assistive duties despite of increased autonomy levels when practicing in an NP role.

P13: ... I thought they had me doing a lot of support work and I thought it was boring. I thought they were not utilizing me as a NP. ...a very well paid scribe...they had me doing a lot of consenting for procedures, and a lot of coordination of procedures... I was managing patients but I felt like a lot of what I was doing I consider RN work, of like even the secretary work, which is the main reason I left it. I saw other NPs working in other parts of the hospital and the clinic... and how they were working and I was like I am not working like that.

P16: ...So she would have me scribe for her, so I would have to go in every room and scribe, and order the tests and do all that stuff...

In summary, participants reported that they did not feel prepared to practice autonomously to the full extent of their scope of practice upon graduation. They were transitioning from a preceptor dependent role of an NP student. Lack of autonomy during 
attending NP school translated into the difficulties with practicing independently upon the graduation.

Table 18

Theme V: Post-Grad: Transitioning to a Greater Autonomy and new Responsibilities. Wide Scope. Autonomy in decision making. Administrative Duties.

\begin{tabular}{|c|c|c|}
\hline $\begin{array}{l}\text { Transitioning to a } \\
\text { greater autonomy } \\
\text { and new } \\
\text { responsibilities }\end{array}$ & Participant's Comment & Summary \\
\hline \multirow[t]{5}{*}{ Wide Scope } & $\begin{array}{l}\text { P1...I feel like I am comfortable but there are still a lot of things } \\
\text { that I see sometimes and I have never seen them before... }\end{array}$ & $\begin{array}{l}\text { Never seen before } \\
\text { things }\end{array}$ \\
\hline & $\begin{array}{l}\text { P5... even to this day when I see people come in with different } \\
\text { stuff I just like oh you know it is you are learning every single } \\
\text { day. I still sometimes wonder what the ... I am doing in this } \\
\text { field, so it is not easy, take one day at a time... }\end{array}$ & $\begin{array}{l}\text { Never seen before } \\
\text { things }\end{array}$ \\
\hline & $\begin{array}{l}\text { P5: ... such a huge learning curve, and the moment you are } \\
\text { comfortable with something, something else comes up or they } \\
\text { change the way you are doing things, and you are just like wait, } \\
\text { hold on you know I am just trying keep my head above water } \\
\text { here... }\end{array}$ & $\begin{array}{l}\text { Never seen before } \\
\text { things }\end{array}$ \\
\hline & $\begin{array}{l}\text { P6: ... it is different responsibility too because you worry, you } \\
\text { want to make sure patient does not have any problems with the } \\
\text { medicine; you want to make sure you give the medicine, the right } \\
\text { thing, you are responsible... }\end{array}$ & $\begin{array}{l}\text { A lot More } \\
\text { Responsibilities }\end{array}$ \\
\hline & $\begin{array}{l}\text { P8: ....now that I am in my professional role I feel like it started } \\
\text { off like a really steep learning curve... kind of switching gears } \\
\text { and figuring out how to handle the different level of }\end{array}$ & $\begin{array}{l}\text { A lot More } \\
\text { Responsibilities }\end{array}$ \\
\hline
\end{tabular}


responsibility.

P9: ...I became a NP, passed my test, got a job, there was no support, I mean you got your license, you walk in, you got your license, I was like ok, you need to go in and see your patients, I mean where was the support, you know... it was a huge transition for me...

P12: ...there are definitely days that I think that the responsibility is overwhelming.

P14: ... very challenging lots of frustration, lots of thinking I chose the wrong profession....

P14:... so there were things that I have never seen, never even experienced even in my rotation that would come in and initially had to call her and actually I did not call her in those cases because most of the time never responded and it was Up-to-date, Google...

Autonomy in decision making
P1: I had a patient that came in she fell and hit her head and she had this huge gash on her forehead, and I knew right away that she needed sutures so I had to send her to the ER or doctors care so that to suture, had I known how to suture I would have been able to do it...

P2: ...give the report to the fellow or the attending tell them about the kid....So I would go and like see the kid and write the note, they would put in a short note that they had seen the kid also cause they were still responsible for them as well, but and the attendings were at some point...

P7: ... lot of autonomy since the beginning, which is nice but also a little scary, not knowing anything bran new NP seeing patients
A lot More

Responsibilities/

Unsupported

Daunting NP

transition

Daunting NP

transition

Never seen before

things/

Unsupported

Scope limitation

Shared Decision

Making

Sudden 100\%

Autonomy 
on your 1st day on your own is pretty intense...

P7: ... in my role as a NP in my current job I actually physically

Prescribing-new

write prescriptions so that was definitely something I had to

skill

learn, ... to figure out how to actually write prescription vs in the

EMR when I was in school I was trained to just click on button

for dosing and send it to the pharmacy directly, so that was a

change from where I went to school and what I currently do in

practice.

P10: ...to learn how to use the nurse and staff or my medtech

Delegating-new whoever was delegated that day to be my support staff, either

skill

nurse or medtech ... I guess it was ok to tell them to go do it

rather than do it myself. And that part has been a bit of a

challenge in learning more delegation I would say.

P11: So in school there was no independent $\mathrm{dx}$ or prescribing, I

Sudden $100 \%$

was writing by everything by my preceptor when I was in school,

Autonomy

and then in practice you know all of the sudden I am handed the

license and I can do it...

P11: I had a ton of autonomy, I probably had too much but

Sudden $100 \%$

luckily it worked out fine...

Autonomy

P12: ...we don't see new patients, the only new once we see is

Limited

hepatitis $\mathrm{C}$, so new patient you are seen by one of the doctors

autonomy/ No

unless you are there for a hepatitis $\mathrm{C}$, then you can see the NP.

new patients

P13: I think they prefer if I don't see new patients, I think they

Limited autonomy prefer if I only saw patients that have been established already...

/No new patients

P14: Prescribing was ...on the go, so there was no... which has

Prescribing-new

been one of the toughest things come in after graduation and

skill 
having to do, especially working in the PC settings...

P15: ...I have trouble delegating just because I don't want to

Delegating-new sound bossy...

skill

P16: ... she told me when I was hired that she wanted to create

Limited

she wanted me to be just like her. And wanted me to practice just

autonomy/mimic

like her and write notes just like her so it was about learning to

the collaborator

do it exactly like her...

P16: ...once I got credentialed and I saw my patients

No Autonomy

independently like she never again like looked at my patients or

During Training

she did not look over my charts or questioned anything. So like

or Credentialing

in that I was like fully autonomous at that point...

Period

Administrative

P2:... there was a lot of pre-op management ...it was a lot of

Management or

Duties.

management, it was like as far as NP job, there was weighted

NP duties/

component on like actual schedule management and OR

Expensive Scribe management...

P6:...I did not have any time to teach the student and so in my

NP Novice is

site I have a lead provider and I was able to go to her and she was

Teaching an NP

in agreement and ye we have to block some of your slots so you

student

have time to teach your student...

P7: ...I precept a lot myself I have actually 3 students now...

NP Novice is

Teaching an NP

student

P13: ... I thought they had me doing a lot of support work and I

Management or

thought it was boring. I thought they were not utilizing me as a

NP duties/

NP. ... a very well paid scribe...they had me doing a lot of

Expensive Scribe consenting for procedures, and a lot of coordination of procedures... I was managing patients but I felt like a lot of what 
I was doing I consider RN work, of like even the secretary work, which is the main reason I left it. I saw other NPs working in other parts of the hospital and the clinic... and how they were working and I was like I am not working like that.

P16: ... So she would have me scribe for her so I would have to Expensive Scribe go in every room and scribe, and order the tests and do all that stuff...

Note. Theme V. Wide Scope. Autonomy in decision making. Administrative Duties.

\section{Theme VI: Embracing the Role and Identity Confusion}

In the sixth interview question participants were asked about their experiences with changing their former professional role to the role of an NP as well as their experiences with the new professional identity during their transition both during attending NP program and after graduation. Data showed that all participants were functioning in a role of an NP during the times of the interviews; however, not all participants transitioned from an RN role. Participants also reported that there was not a clear separation between the roles of an $\mathrm{RN}$ and a provider. Additionally, results showed that transitioning to the role that requires the change of the mindset from taking orders to giving orders was challenging as participants felt underprepared for the role. Though the transition was described as difficult, some participants expressed embracing their role and using all available resources to adjust to their new responsibilities.

Pre-graduation: Embracing the role and identity confusion. When asked about changing roles during the transition time prior the graduation, participants reported that their transition to the role of an NP student was not difficult, "kind of seemed more normal to you at the time." Many reported working as an RN while attending NP school. Some participants 
reported it was helpful for their role learning to continue practicing in an $\mathrm{RN}$ role while attending NP school, whereas others reported it to be confusing. Several participants reported never working in the role of an RN prior their direct entry NP program admission. They reported having to progress from their non-nursing role to receiving their Bachelors, Masters and NP degrees (see Table 19).

P6: I was working still as a nurse and still had an access to patients all the time, still be able to, you know, even though I was not working as an NP role but still been able to see patients and assess them, talk to them, look at their you know and start making connection that way too. As far as a big picture, I am not in the provider role, but you know I can understand why this patient has this based on how they presented or... Though transition to the role of an NP student was not reported as difficult, transition to practice was reported to be challenging. Participants reported that when transitioning to the role of an NP student, they did not have a realization of NP role autonomy and new responsibilities the way they did after graduation when they had to start practicing "right out of the gate," taking full responsibility for patient care outcomes.

Post-graduation: Embracing the role and identity confusion. When asked about the role change during the transition time after graduation, five major categories emerged from data including transitioning from an $R N$ role, transitioning from a non- $R N$ role, blurred nurse Iprovider lines, pre and post-graduation reality match, and transition from the 'notify the provider' mindset (see Table 19). 
Transitioning from an $R N$ role ing. Though many expressed difficulties transitioning to a new NP role, most participants reported being glad to leave $\mathrm{RN}$ role. Some were happy to leave $\mathrm{RN}$ role, describing it as more of a task oriented, the kind of role that included many assistive type of duties. Many welcomed an exciting possibility of an increased autonomy of practice that would come with advancing to an NP role.

P6: I think it was exciting ... I won't have to do this that much often, you know, some of the things in nursing that you kind of got tired of or burn out of, you knew your role was changing...

P16: I really loved it to be honest with you, because nursing after so long ...I felt like a waitress, like I would just be taking people stuff and carrying out orders, it was not like decision making ... I really enjoyed transitioning to a NP because now I got to be the one saying, this is what needs to happen...

Rude awakening. Post-graduation excitement was reported to quickly change to a realization of new responsibilities of a new role combined with feelings of being unprepared to practice independently to a full extend. Advancement form a familiar and less autonomous RN role to a less familiar and more autonomous NP role was compared to learning a new language or as "rude awakening." One participant who advanced from a role of a paramedic; however, expressed that his transition to a new role was not as abrupt as he was used to practicing independently.

P5: I was actually scared to death to go practice ... I stayed working as an RN for about a year before I even started applying for NP positions. 
P11: ...but in this new job I have often felt like, o my Gosh, like I just want to get a job as a nurse, like this seems overwhelming how much, it is hard because physicians they get residency programs and they like get a ton more training I feel like than we do as NPs, they get more.

$R N$ and NP roles. Though graduated with the new NP degree, some participants reported still working in an $\mathrm{RN}$ role (often for a year or longer) after graduation; they reported that inability to find NP job, being afraid to transition to a new role, RN job stability, or personal life transitions precluded them from making profound changes advancing to their new role. At the same time, some informants reported practicing in both NP and RN roles. Practicing in both roles was described as strange as informants had to constantly change form lesser autonomy in the decision making process to more autonomy.

P16 ... when I transitioned to NP, I still worked as a nurse, so for the first whole year that I was an NP... that was really odd because I would go from I am the one making the decisions and ordering stuff during the week on patients too, now on the weekend I can't do any of that, I am just like following orders, so it was really weird to live in both of those worlds at the same time.

Longing for an $R N$ role. While advancement from an $\mathrm{RN}$ role was identified as a positive change, most participants were also longing for their old and comfortable RN role. Participants stated that when they realized the new role's responsibilities (at the start of the new NP job), they were often day dreaming of returning to their "safer" RN role. 
P11...o like starting this new job I often thought to myself, Gosh it was so much easier to just be a nurse...

Though participants dreamed about going back to their RN role, they stated that they were unable to do so due to the investments they have already made into the new NP role.

P11: There were many times when I thought I just go get a job and life would be so much easier and simpler; I could just be an RN but I don't do it because I went to school and I spent all this time, likely I don't have debt, but you know a lot of people push forward because they are in debt and they don't have a choice.

P12: ....it is a lot more responsibility...there are a lot of days I day dream about being a nurse again and not being quite responsible for the overall outcome of patient... P13: ... may be it would be safer if I stayed doing that but I already spent all this money on that school so I guess, I better do the other thing that I have now been trained for... P14:...very challenging lots of frustration, lots of thinking I chose the wrong profession... some days I told my husband if I am going to leave the field, which is, I am questioning because I spent so many years and a lot of money and a lot of time... Participants were reporting missing the relationships they were able to establish with their patients in the past having sufficient amount of time with their patients in an $\mathrm{RN}$ role as opposed to the short appointment time slots they had with their patients now. They also missed the time when they did not have to take their work home; in their new NP role, they would often have to take charting or patient follow up duties home. 
P11: When you are a provider like you are trying to sometimes you are trying to figure out those diagnoses when you get home, sometimes you have to chart when you get home...

Using RN knowledge base. Though many reported the two roles of an RN and NP to be different, they also reported that their RN experience was helpful in their changing to a new role. Namely, the ability to connect with the patients and develop nurse patient relationship was reported to be a nursing skill that most participants took with them into their NP role.

P4: ...my certification is family, so I went into a specialty, right into my first job but I did have a background, as an RN I worked in women's health in the hospital, so I was able to carry that experience as well.

P6:... when I felt nervous or not competent I kind of use my nursing experience because I know I am a nurse so I know I can interview patient, I know I can assess the patient, you know, I just have to get the diagnosis and the plan down and filling the prescriptions and all that so I just focused on the things that I knew I could do well... things I was not sure about I just made sure I looked them up or you know had access to my resources...

Transitioning from a non-RN role. Two participants reported transitioning to an NP role from non-RN roles through enrolling into a direct entry nursing program. These participants quickly progressed to the BSN degree and then proceeded with the graduate nursing degree. One participant, P14, transitioned from finance; this participant has never worked as an RN until the graduation from an NP program; after graduation this participant took both jobs, NP and RN, thus transitioning to both roles simultaneously. The second participant, P10, transitioned from 
being a paramedic; this participant has never worked in a role of an RN. Role transition from being a paramedic was reported easier when compared with transition from $\mathrm{RN}$ or non-health related professions.

P10: ...we were not limited to fixed protocols or fixed guidelines...the drugs that we had, we were expected to know how they work, how they interact with other drugs, and we were able to do what was necessary for our patient as long as there was an evidencebased reason for it...rather than calling us ER medical technicians, we would say pre hospital providers, to reflect the education, training we had.

P14: ...the transition from NP student to NP role was more difficult than transition being in finance to working in health care. I thought it was not going to be that way. I felt like I got more support more training, more everything, more money spent on training to watch people's money than I am getting to watch people's health.

Blurred provider-prescriber lines. Participants reported no clear separation and some confusion about the roles of an RN, NP, and a medical doctor. Additionally, confusion about NP role was reported to be among patients and other non-NP providers. As data showed, it was confusing for staff to perceive a nurse who they always knew as a nurse, in her new advanced role. Additionally, some reported that as opposed to the MD role, they were at times expected to provide nursing care in addition to their provider duties, which was difficult due to the time constrains of the provider's role. Data also showed that it was hard for participants to not perform "nursy" duties that they were accustomed to in addition to their provider's responsibilities. However, when practice had more than one NP, the role was better understood 
by staff and providers. Unclear role boundaries led to confusion about the scope and functionalities of the role.

P2: It is sometimes hard when you ...stay in the place that you worked as a nurse ... where you knew people who knew you as a nurse, because it was hard for them to see you in that role or for you to ask them to do something for you when you used to be a nurse...

P5: O man...that is still something that I am working on dividing ...I mean there were times where if I am doing a diabetic foot check or something like that, you know, and the person is kind of slow to get their shoes and socks off, $\mathrm{RN}$ in me like jumps, unties their shoes, reaps off their socks and, you know, and then even after the fact I want to put them back on and fit their shoes back...so I feel there is a part of me that still...there is so many things I jump in just to do the RN job ... vital signs, or swab their throat for strep, or whatever it you know the medical assistant or RN would be doing ... I am thinking in the environment that I am in it is almost expected ... there is no way an MD would do all this. So, it is kind of the lines are still little blurred... I don't mind doing it here and there but if I am doing it all day long, I am like thinking ok wait, you can't just take over my duties, you know so, and I don't think sometimes there is a whole lot of thought goes into when you are prescribing, assessing, when you are diagnosing you are you really need to have your thought process clear and not bugged down with all these task oriented things... 
P5: ...I don't think necessarily the role is well understood even in, you know, among your colleagues, you know, because they still think well you can do all the RN stuff... P6: I was lucky I already had NPs, so I did not have to explain my role to the staff. P15: ...probably the biggest challenge in that because we are nurses, it is hard to not try to still do "nursy" things and try to help out like our support stuff...

Further, data showed that some participants did not see themselves as an advanced nurse but rather identified with the role of an advanced provider. They also noted that NP role is closer to medicine than nursing because of the ability to diagnose and prescribe. However, the ability to build nurse-patient relationships was reported as carried over to an NP role.

P10: I would say I am a paramedic who became an advanced practice provider...

P16: I feel like I am doing medicine, completely, I don't feel like nurse anymore like I am the one, I am critically thinking, and ordering things and doing things ... I am the... one determining what needs to be done and doing it, it is way more close to the medicine than nursing.

Participants reported that other providers and patients lacked clear understanding of the role. Participants stated that because of this lack of understanding of their role, many providers, including medical residents and attending MDs, expected them to practice independently right out of school.

P12: And then there are days that may be some patients don't think of you on the same level as like provider... why did you quit half way, people that don't understand the role 
$\ldots$ it is like the NP like a doctor is half way ... it is frustrating that ... you are not fully recognized by some patients as a confident provider...

P13: I felt like my role was very ill-defined and the residents did not know how to act towards me, and I did not know how to act towards the residents and it was very uncomfortable, to be quite frank.

P14: I mean, some of them you tell them, ok this is what NPs do and they sound shocked, o really...they look at you like a resident you know fully prepared .... and you should be happy and I am like that is not how we work, so all of that kind of like left me with a long emotional distress.

Pre and post-graduation reality match. When participants started practicing in their new NP role, they realized that their role expectations did not match the reality. First, participants reported a much harder transition to practice than they expected; it "was a rude awakening." Second, participants were expected to be able to practice independently; however, they felt underprepared to do so after graduation. They relied on others to help them with their transition, but instead they encountered unrealistic expectations of their role.

P1: and then I was permanently to our expended pediatric department, which I had no experience with peds other than school, so I was terrified and I still did not get a preceptor, so I pretty much just had to figure out on my own.

P10: I don't feel necessarily prepared to be an independent provider and the reason I chose the NP route was to be independent. 
P11: I felt like our program was not very strong in helping you to like really learn and know inside and out the things you would need to know for clinical...

Third, participants stated that their training had limitations, including lack of procedure training, lack of clinical experiences, lack of laboratory tests ordering and interpretation, and lack of differential diagnoses training.

P10: I work in urgent care and there was not a lot of procedural training in my program. P14: I mean some of them you tell them, ok this is what NPs do and they sound shocked, o really...

P14: no hands on, I think that is where my training I felt lacking because I ended up in ED having to suture, and did not even have a suture class, which I was really surprised... Participants reported lacking deeper fundamental knowledge that is required in their places of practice. Participants reported that they would need to practice for some time before they could build the necessary knowledge base. Additionally, participants struggled when encountering other professionals' lack of knowledge about the NP role. The lack of role understanding among the professionals appeared to impede interdisciplinary communication.

P14: ...unfortunately my experience with before graduation and after graduation kind of seem like opposites, just because I was trained with NP but I have been working with MDs... it was a different experience being trained by NPs and then going out working with not many NPs and realizing that ... your expectations and their expectations are completely different. 
Transition from the 'notify the provider' mindset. Participants reported that the biggest part of their role transition was related to changing their mindset from someone who reports to the doctor and takes doctor's orders to someone who takes responsibility for making critical patient care decisions. They stated that this new mindset is "heavy" and frightening because the lives of their patients are in their hands. However, participants also noted that they enjoy their new responsibilities and autonomy of their role.

P4: ... it was very overwhelming, because ... as a RN you can always kind of depend on somebody to go behind you, like everything was not on you, but now I feel like everything is you know on me...

P15: it is a little bit more stressful just knowing that these people lives in your hands, you could make mistake that could affect their health, so it is a big transition to go from you know a just a RN role and to making those decisions.

In summary, before the graduation, participants experienced minimal role change. Many stated that they still worked as RNs. Some noted that working as an RN was helpful in learning the NP role; however, many stated that it was confusing. After graduation, participants realized that their role was not well understood by others; they experienced that others' expectations of their role were different form theirs. Additionally, participants reported difficulties from dissociating from their RN role into the prescriber mindset. NP role was often noted to be closer to medicine than nursing. 
Table 19

Theme VI. Embracing the Role and Identity Confusion.

\begin{tabular}{lll}
\hline $\begin{array}{l}\text { Embracing the Role } \\
\text { and Identity }\end{array}$ & Participant's Comment & Summary \\
Confusion & & \\
\hline Pre-graduation: & P6: I was working still as a nurse and still had an access to & RN Work While in \\
& patients all the time, still be able to you know even though I & NP school \\
& was not working as an NP role but still been able to see & \\
& patients and assess them, talk to them, look at their you know \\
& and start making connection that way too. As far as a big \\
& picture, I am not in the provider role but you know I can \\
& understand why this patient has this based on how they \\
& presented or...
\end{tabular}

Post-graduation:

Transitioning from

$\mathrm{RN}$ role or "rude

awakening"
P4: ...my certification is family so I went into a specialty, right into my first job but I did have a background, as an RN I worked in women's health in the hospital, so I was able to carry that experience as well.

P5: I was actually scared to death to go practice, I was actually going through .... at that time as well and I stayed working as an RN for about a year before I even started applying for NP positions just because I felt I was going through so many other changes that I am like I did not feel like I was going to be up for it...

P6: I think it was exciting...I won't have to do this that much Glad to move on often you know some of the things in nursing that you kind of got tired of or burn out of you knew your role was changing...
Using RN knowledge

base

Rude awakening 
P6:... when I felt nervous or not competent I kind of use my

Using RN baseline nursing experience because I know I am a nurse so I know I knowledge can interview patient, I know I can assess the patient, you know I just have to get the diagnosis and the plan down and filling the prescriptions and all that so I just focused on the things that I knew I could do well... things I was not sure about I just made sure I looked them up or you know had access to my resources

P8: I feel more like a provider now, am but I sometimes like Longing for an $\mathrm{RN}$ really miss being a nurse.

role

P9: ...I was ready to transition from being an RN to NP

Glad to move on because I was not overly excited with some of my RN from an $\mathrm{RN}$ role positions...

P11:...o like starting this new job I often thought to myself, Longing for an $\mathrm{RN}$ Gosh it was so much easier to just be a nurse and like you show up to work and you do your job and you go home and role/taking work like you don't have to think about it anymore ... It is not really like that when you are a provider like you are trying to sometimes you are trying to figure out those diagnoses when you get home, sometimes you have to chart when you get home and sometimes you have to like you know follow up on lab results when you get home because the culture comes back negative or positive and you have to do something with it...

P11: ...but in this new job I have often felt like o my Gosh, Longing for an $\mathrm{RN}$ like I just want to get a job as a nurse, like this seems role/underprepared overwhelming how much it is hard because physicians they get residency programs and they like get a ton more training I feel like than we do as NPs, they get more. I never even learned how to interpret an X-ray, I barely drew any labs as a 
student and interpreted them, like I just feel like I am in new foreign land that I am having to learn the language completely all over again.

P11: there was many times when I thought I just go get a job and life would be so much easier and simpler; I could just be an RN but I don't do it because I went to school and I spent all this time, likely I don't have debt, but you know a lot of people push forward because they are in debt and they don't have a choice.

P12: ... it is a lot more responsibility and sometimes it can be overwhelming, I mean there are a lot of days I day dream about being a nurse again and not being quite responsible for the overall outcome of patient...

P12: ... graduated a long time ago in 2011 and then I worked I did not start working as a NP till $2016 \ldots$

P13: ... may be it would be safer if I stayed doing that but I already spent all this money on that school so I guess, I better do the other thing that I have now been trained for but it was easy to feel like I wanted to stay with the safety of the position I already knew how to do.

P14:...very challenging lots of frustration, lots of thinking I chose the wrong profession... some days I told my husband if I am going to leave the field, which is I am questioning because I spent so many years and a lot of money and a lot of time and all that and even with this has not been ye I am still very new in the industry and I am still taking it one day at a time but some days I still not sure that I made the right choice, it has been very emotionally draining experience.
Too much invested to go back to $\mathrm{RN}$ role

$\mathrm{Rn}$ work after the np graduation

Too much invested to go back to $\mathrm{RN}$ role

Too much invested to go back to $\mathrm{RN}$ role 
P16: I really loved it to be honest with you, because nursing after so long ...I felt like a waitress, like I would just taking people stuff and carrying out orders, it was not like decision making ... like you could call a physician and say hey this is what is going on and this is what I think needs to happen but ultimately it was a physician's decision you know what they did and so it was I really enjoyed transitioning to a NP because now I got to be the one saying this is what needs to happened ... so that like it really gave me, a lot of enjoyment to be able to go from just completing somebody else's orders to being able to critically think like ok what would be the best for this patient...

P16 ...I also like when I transitioned to NP I still worked as a nurse so for the first whole year that I was an NP... So I worked as an NP during the week and I worked as a nurse on the weekends and that was really odd because I would go from I am the one making the decisions and ordering stuff during the week on patients too, now on the weekend I can't do any of that, I am just like following orders, so it was really weird to live in both of those worlds at the same time.

Transitioning from a Non-RN role
P10: I worked as a paramedic and straight through from getting my RN into my MS in NP school and I did not work as a $\mathrm{RN}$.

P10: ...we were not limited to fixed protocols or fixed guidelines...the drugs that we had, we were expected to know how they work how they interact with other drugs and we were able to do what was necessary for our patient as long as there was an evidence based reason for it.
Glad to move on from an $\mathrm{RN}$ role

Rn work after the np graduation/odd living in both worlds

Transitioning from a Non-RN role (paramedic)

Transitioning from a Non-RN role (autonomous paramedic role) 
P10: ...rather than calling us ER medical technicians, we would say pre hospital providers, to reflect the education, training we had.

P14: ...the transition from NP student to NP role was more difficult than transition being in finance to working in health care. I thought it was not going to be that way. I felt like I got more support more training more everything, more money spent on training to watch people's money than I am getting to watch people's health.

P14: November 2016 I started working as an RN FT and then December 2016 I started working as an NP per diem in the ED

P14: At least you have it on your resume that you worked as a nurse because everybody seems to have that bias that you need to work as a nurse before you can work as an NP.

Blurred provider/prescriber lines.
P2: It is sometimes hard when you ...stay in the place that you worked as a nurse ... where you knew people who knew you as a nurse, because it was hard for them to see you in that role or for you to ask them to do something for you when you used to be a nurse...

P5: O man...that is still something that I am working on dividing ...I mean there were times where if I am doing a diabetic foot check or something like that you know and the person is kind of slow to get their shoes and socks off, $\mathrm{RN}$ in me like jumps, unties their shoes, reaps off their socks and you know and then even after the fact I want to put them back on and fit their shoes back... so I feel there is a part of me that still...there is so many things I jump in just to do the RN job
Paramedics-pre-

hospital providers

Transitioning from a

Non-RN role/difficult

Transitioning from a Non-RN role to $\mathrm{RN}$ and NP roles

no $\mathrm{RN}$ job experience bias

changing places when changing roles

Blurred RN/provider lines/doing medicine not nursing 
... VS, or swab their throat for strep or whatever it you know the medical assistant or RN would be doing ... I am thinking in the environment that $\mathrm{I}$ am in it is almost expected ... there is no way an MD would do all this. So it is kind of the lines are still little blurred... I don't mind doing it here and there but if I am doing it all day long I am like thinking ok wait, you can't just take over my duties, you know so, and I don't think sometimes there is a whole lot of thought goes into when you are prescribing, assessing, when you are diagnosing you are you really need to have your thought process clear and not bugged down with all these task oriented things...

P5: ...I don't think necessarily the role is well understood even in you know among your colleagues you know, because they still think well you can do all the RN stuff...

P6: I was lucky I already had NPs so I did not have to explain my role to the staff; I had to explain my role to patients...

P7: it is challenging even to tell people that I am an NP, most people still don't realize ... what I can do basically and a lot of people will ask me if I am going to school to be a doctor, or if they should call me a doctor...

P10: I still feel like a paramedic, and I still like practice medicine, I did not work as a nurse, I did not spent any professional time as an RN and that I don't mean that in anyway disrespect, I respect nursing. I had a fantastic job that I really enjoyed and I really loved, I left that with the goal of practicing medicine. I have been an advanced practice provider, I ... invested in choosing nursing route vs. the PA route so long as looking at practice model ... nurses have
Blurred lines-role is poorly understood

Role is better understood when employer has more than $1 \mathrm{NP}$ Blurred RN/provider lines-identifying with being a provider not a nurse/doing medicine not nursing 
independent practice ... so I don't really feel like I have the nurse identity ... if you put words you would use to describe myself, I would say I am a paramedic who became an advanced practice provider... I just I feel like the training and the job I do to care for the patients, I try to bring evidenced base medicine to them.

P12: And then there are days that may be some patients don't think of you on the same level as like provider where they like how come I never see the doctor, or why do I always see a NP or why did not you finish school, why did you quit half way, people that don't understand the role ... it is like the NP like a doctor is half way ... it is frustrating that ... you are not fully recognized by some patients as a confident provider ...

P13: I felt like my role was very ill defined and the residents did not know how to act towards me and I did not know how to act towards the residents and it was very uncomfortable to be quite frank.

P14: I mean some of them you tell them, ok this is what NPs do and they sound shocked, o really...they look at you like a resident you know fully prepared ... and you should be happy and I am like that is not how we work so all of that kind of like left me with a long emotional distress.

P15: ...probably the biggest challenge in that because we are nurses, it is hard to not try to still do "nursy" things and try to help out like our support stuff...

P16: I feel like I am doing medicine, completely, I don't feel like nurse anymore like I am the one, I am critically thinking, and ordering things and doing things, like I don't feel like a
Blurred RN/provider lines/ explaining the role to patients

Blurred RN/provider lines/explaining role to medical residents

Blurred RN/provider lines/explaining role to medical residents

Blurred RN/provider lines/hard to not do "nursy" things

Doing medicine not nursing 
nurse anymore. I feel like a nurse was just ... the orders and the work of someone else but now I am the... one determining what needs to be done and doing it, it is way more close to the medicine than nursing.

Pre and postgraduation reality match

P1: and then I was permanently to our expended pediatric department, which I had no experience with peds other than school so I was terrified and I still did not get a preceptor, so I pretty much just had to figure out on my own.

P6: Pts I ended up encountering as a new NP may be a few of them matched the patients I saw at my clinical setting.

P10: as far as the clinical role after graduation I definitely don't feel as if I was well, I think I was very well trained for one particular job but that is not the type of a job necessarily, the type of work that I do. I don't really feel adequately prepared for that part...as an NP student I was trained in family medicine, I think I definitely had clinical preparation for primary care and clinical management but I work in urgent care and there was not a lot of procedural training in my program.

P10: I don't feel necessarily prepared to be an independent provider and the reason I chose the NP route was to be independent.

P11: I felt like our program was not very strong and helping you to like really learn and know inside and out the things you would need to know for clinical... like the common things you would need to do and I think that that really hurt me ... I have added on general pediatrics and I feel completely

\begin{abstract}
No preceptor/placed in the area of peds with no prior work peds experience
\end{abstract}

Different from school

Lack of procedure training which is required for the job

Did not feel prepared to function independently as expected

Did not have depth of knowledge as expected and necessary for practice 
unprepared for it, because I feel like I did not really learn the stuff inside and out that I needed now in school ...

P14: ...unfortunately my experience with before graduation and after graduation kind of seem like opposites, just because I was trained with NP but I have been working with MDs... it was a different experience being trained by NPs and then going out working with not many NPs and realizing that ... your expectations and their expectations are completely different.

P14: no hands on, I think that is where my training I felt lacking because I ended up in ED having to suture, and did not even have a suture class, which I was really surprised...

P16: I kind of felt I felt that school did not prepare me all the way to be honest with you like I got out and felt like OMG I have got to do this now.

Transition from the 'notify the provider' mindset.
P4: ... it took a minute to get used to, one just being in that role, of big a provider I think, you know going from following orders to ... talking with staff and tell them what to do...

P4: ... it was very overwhelming, because $\ldots$ as a $\mathrm{RN}$ you can always kind of depend on somebody to go behind you like everything was not on you, but now I feel like everything is you know on me...

P4: ...though I was working in a role of an $\mathrm{RN}$ your mind is now thinking like a provider; like things that you would not look at as an $\mathrm{RN}$ you start to kind of key in on.. P8: transitioning to be a person that makes the really
Worked exclusively with NPs while in school; after graduation had to work exclusively with MDs and residents, was unprepared Underprepared to perform procedures

Underprepared Transition to giving orders

Overwhelming responsibilities

Started role change while in RN role/helpful Overwhelming 
important decisions it is hard; it causes me like a lot of anxiety and a lot of worry after work...

P13: I thought it was kind of hard honestly, because there was as a nurse you are expected to take orders ... and when you are in school you are sort of in this weird limbo where you are like trying ...so when I was at work I remember trying to think about o if I was a provider I think I would do this and like but you did not want to say anything ... like my brain was trying to go there, and unfortunately my mouth followed it... but I don't know it was kind of a bit of a difficult transition I think.

P15: it is a little bit more stressful just knowing that these people lives in your hands, you could make mistake that could affect their health, so it is a big transition to go from you know a just a RN role and to making those decisions.

P16: nursing to me was kind of like ... almost like a waitress, I don't mean that in a derogatory term but it was a lot like doing other people's orders... it was not a lot of critical thinking needed ... like getting somebody this, getting somebody that... to me the nurse role and the NP role are completely different. NP role is more like what the doctor is doing to be honest with you, I think like the nursing ... NPs relate to patients differently than doctors do but we are doing the doctor's role, we are like prescribing, and diagnosing and taking care of meds, I think that NP role is closer to a physician role than to an $\mathrm{RN}$ role. responsibilities

Started role change while in $\mathrm{RN}$ role/not helpful

Overwhelming responsibilities NPs' job is doing medicine not nursing

Note. Theme VI. Embracing the Role and Identity Confusion 


\section{Theme VII: Transition Reactions. Excited and Unfulfilled}

In the seventh interview question participants were asked about their emotions and reactions to the role transition process both during them attending NP program and after graduation.

Pre-graduation: Transition reactions. When asked about the reactions and emotions participants experienced prior the graduation, they identified two major categories of negative emotions and normal emotions (see Table 20).

Negative emotions. Participants expressed that finding and securing clinical sites was the most stressful and upsetting element of their role transition while attending school. They reported feeling frustrated, desperate, overwhelmed, and stressed out by the process of finding sites. Additionally, participants reported that it was frustrating to frequently change preceptors and clinical sites. While they were happy to learn different providers' styles and observe how various clinical settings function, they were upset about not having an opportunity to appropriately adjust to the site and the preceptor. Difficulties with finding and securing clinical learning environments along with frequent changes of sites and preceptors appeared to impede role learning and caused participants experience many negative feelings.

P4: ...I was googling something, just so desperate to find preceptor...very frustrating, even thinking about it makes me mad...

P8: I would say it was probably the single most stressful thing about school because if you did not find a preceptor, nobody was going to help you and you would have to sit out a year at school; that was stressful. 
Normal emotions. Participants reported that the following school related events caused them to feel normal emotions. These events included writing papers, deadlines, tests, certification exam, and clinical experiences. Participants reported that they expected help with transition upon the graduation, so they did not worry about their role transition when attending school. Participants reported feeling proud and excited after the completion of the program until starting a new NP job when feelings of excitement and pride were replaced by the realization of being unprepared for the role.

P1: I expected...I was thinking I was going to have all the support and help after I finish but that was not the case so...I did not feel as the nervous ...most of those nervous feelings were toward trying to pass the certification.

P11: I was not stressed out or anxious at all when I was an NP student, because the responsibility was not on me and now the responsibility is on me.

Table 20

Theme VII: Pre-graduation: Transition reactions.

\begin{tabular}{|c|c|c|}
\hline $\begin{array}{l}\text { Transition } \\
\text { reactions or } \\
\text { rude } \\
\text { awakening }\end{array}$ & Participant's Comment & Summary \\
\hline $\begin{array}{l}\text { Negative } \\
\text { emotions }\end{array}$ & $\begin{array}{l}\text { P4: ...I was googling something, just so desperate to find } \\
\text { preceptor...very frustrating, even thinking about it makes me mad... }\end{array}$ & $\begin{array}{l}\text { Frustrated with } \\
\text { looking for sites- } \\
\text { mad }\end{array}$ \\
\hline & $\begin{array}{l}\text { P5: A disaster... it was really tough because the school I was that they } \\
\text { did not have a whole lot of contracts with clinics... }\end{array}$ & $\begin{array}{l}\text { Frustrated with } \\
\text { looking for sites-a } \\
\text { disaster }\end{array}$ \\
\hline
\end{tabular}


part of my schooling because it was very hard to find people that were willing to precept...

P7: ...change in clinicals so new preceptors every couple months, so I Lack of selfdefinitely felt unsure of myself and in terms of what was expected from different preceptors in clinical rotations, the confidence, I feel when I confidence due to start to build my confidence up with my preceptors I transitioned to frequent site different clinic and it kind of start not back from ground zero but just ... basically with every rotation...

P8: I would say it was probably the single most stressful thing about Stressful-fear of not school because if you did not find a preceptor nobody was going to help finding a site you and you would have to sit out a year at school; that was stressful.

P9: Stressful I had to find everybody by myself and school did not provide any help and I literally called tons of clinics until somebody says yes, it was very horrible.

changes

Stressful-lack of assistance with finding sites

P15: I did have a few people in my group that really I mean I heard that Dropping out of were really challenged in finding preceptors and one ... had to drop out school of our program because she was in the area that was very limited with preceptors and she was not able to find a pediatric provider...

Normal P1: I expected...I was thinking I was going to have all the support and Only nervous about emotions. help after I finish but that was not the case so ...I did not feel as the nervous ...most of those nervous feelings were toward trying to pass the certification, I don't think I was thinking about how it will be practicing after graduation. I did think I will have a lot of support, I thought I school since thought will have a lot of support after graduation would at least have 90 days to have a preceptor to kind of help me so I guess I just had too many expectations of how it would be when I start at my 1 st job. 
time it is exciting to start something new but scary at the same time...and when you start getting it there is a sense of pride.

P11: I was not stressed out or anxious at all when I was an NP student, because the responsibility was not on me and now the responsibility is on me. to start something

new

Not stressful due to the responsibility is on preceptor

Note. Theme VII. Negative and normal emotions.

Post-graduation: Transition reactions. When asked about the reactions and emotions participants experienced after graduation, they identified two major categories of negative emotions and positive emotions (see Table 21 and Table 22). Participants reported mostly positive emotions after graduation and right before entering the practice. They reported mostly negative emotions after entering practice.

Table 21

Post-graduation: Transition reactions.

\begin{tabular}{lll}
\hline $\begin{array}{l}\text { Type of } \\
\text { Reactions }\end{array}$ & Reactions and feelings & Reported causes for reactions and feelings \\
and & & \\
feelings & & \\
\hline Negative & overwhelmed & patient volume \\
& angry & inconsistencies of learning from different providers \\
& loubtful & lack of job orientation \\
& emotionally drained & lack of structured job orientation when one was offered \\
& unfulfilled & finding a job \\
& nervous & feeling unprepared to function in an NP role \\
& scared & being fearful of making a mistake and doing a good job \\
& lafraid & lack of clinical training and knowledge \\
terrified & & role confusion and having to explain role to others
\end{tabular}




\begin{tabular}{ll}
\hline $\begin{array}{l}\text { frustrated } \\
\text { anxious }\end{array}$ & graduating with entry level degree but having to assume full \\
alone & autonomy and responsibility for complex care right after the \\
& graduation \\
& the amount of self-learning involved \\
& working alone with no guidance or support \\
& passing boards \\
& \\
excited & completing school \\
proud & achieving their educational goals \\
relieved & learning new things \\
& gaining a new role \\
& getting a new job \\
& having an increase in autonomy of decision making \\
& leaving RN role \\
& leaving inconvenient weekend schedules and inpatient environment \\
& having an increase in salary \\
& gaining ability to make a greater impact in people's health \\
&
\end{tabular}

Note. Theme VII. Negative and positive emotions and reactions.

Table 22

Post-graduation: Transition reactions and feelings.

\begin{tabular}{|c|c|c|}
\hline $\begin{array}{l}\text { Transition } \\
\text { reactions } \\
\text { and } \\
\text { feelings }\end{array}$ & Participant's Comment & Summary \\
\hline $\begin{array}{l}\text { Negative } \\
\text { emotions }\end{array}$ & $\begin{array}{l}\text { P1: Kind of overwhelmed and wondering if I made the right decision, } \\
\text { which I think I did, but at first you get kind of ...it is so new to you and } \\
\text { you like what did I...Sometimes I felt overwhelmed because I am the } \\
\text { only person there by myself especially after five, so that was really } \\
\text { nerve-racking because I stayed at work until eight. So I was very scared } \\
\text { that I would miss something or do something wrong just form being }\end{array}$ & $\begin{array}{l}\text { Overwhelmed, } \\
\text { doubtful scared due } \\
\text { to being alone }\end{array}$ \\
\hline
\end{tabular}


there by myself. When I was there in the day time the first you know with other providers, it was a little more comforting feeling, especially during the winter months of my first year it was very busy. I ended up seeing like up to 40 patients one time and I almost went home and cried, to my husband, he is like it is ok, you are done, it is over, you did it...

P2: Terrifying ..silence... because all of the sudden you have all of these liabilities you have not had before.

P3: It could be stressful because we see too many patients

P4: for me the most overwhelming part is having to consult with the referring provider...

P4: ... overwhelming very very, you feel like you are going fail or do something wrong...

P5: ... when I actually got out of school and ... I was certified and that was, I was scared to death, I was you know I literally ... I did not even look for a NP job for about a year...

P6: there was some frustration at times because just depending on like when I started I was not every day I would have the same person to reach out for help or collaborate with, so those days rotating I did not know the person well, that could be a little bit frustrating and then you don't want to feel like you are holding that person down because they have to see patients too so you did not want to place extra burden on them to ask some questions and disturb their day.

P7: I was nervous, I was afraid; I mean I was afraid missing a diagnosis or making a mistake... lot of autonomy since the beginning, which is nice
Terrified due to increased liability

Stressed due to high patient volume Intimidated Overwhelmed due to fear of making a mistake

Scared

Frustrated having to learn from different providers and be a burden to them and not to know them well enough to learn from them

Fear of making mistake/doubtful/ 
but also a little scary, not knowing anything bran new NP seeing patients on your first day on your own, is pretty intense, but it worked out for me.

P8: ... you are expected to get from 1 in hour to 2 in hour, it was like I was working one day and the lead doctor came up to me and said what are you thinking about going to 2 an hour tomorrow ha, I was like ...I was not mentally prepared ... to do that.

P11...seems overwhelming ... it is hard because physicians they get residency programs and they like get a ton more training I feel like than we do as NPs... I never even learned how to interpret an X-ray, I barely drew any labs as a student and interpreted them...because of the breadth of knowledge that goes in that and me feeling like I was not adequately prepared.

P11: I have seen them treat like 27 patients a day and that stresses me out because I am like how am I going to do it...that is what really more than anything, the load and the fear of missing something is really big too.

P12: I have a lot more days where I am like o man, I like over my head, like questioning if I feel like I know enough...

P13: I am finally to the point when I am very comfortable being a nurse and I feel like I am good at it ...I have to become good at this new thing, which was kind of like it was very nerve racking; I ... looked for a job that I thought would have low expectation of me and then at least they were understanding that I am a new graduate and I would need help...

P14: can you imagine killing somebody unintentionally, accidently because you did not get the right support; that left me very stressed out, very unfulfilled in my role as an NP, I thought I was going to be coming in making a world of difference... I feel alone... lots of frustration, lots of thinking I chose the wrong profession. wanting to do the

right thing for patients Unprepared to increase patient volume Overwhelmed due to lack of training and having to learn on own

Stressed due to high patient volume Intimidated

\section{Self-doubt}

nerve racking

Stressed out, unfulfilled, alone, fearful of making a mistake 
P14: ...my experience has left me ... with more, I feel like more self-

Self-doubt doubt, then I have ever felt before ...I doubted I still do, I doubt myself a lot, I am unsure if I making the right decision because my training had not been consistent and I just usually pray every time I go to work that I am doing the best for my patient.

P15: I was kind of angry because of the way he treated me... we had our Angry-collaborator meeting he was saying you know some of my deficiencies for physical treatment exams and those kind of things... I said you know I asked you for help in those areas and you taught me nothing...

P15: ... going into my first job as a new NP, first the transition part is a Frightened of little frightening going from having the security of your preceptors and suddenly increased making the decisions and they are not falling on your shoulders or on liability your back, or on your license, and going into practice when you realize that you have all this responsibility making decisions...

P15: ...mostly stress you know just to make sure that I am doing the Stressed right thing...

P16: ...I really had to learn on my own, which was kind of scary, the Scared because of new NP because I was bluntly aware that I did not go to medical school and some of the patients that I was having to take care of were really having to learn on really sick...

her own

P16:I literally prayed every day before I went to work, Lord don't let me Afraid of making a hurt somebody today, give me the wisdom to take care of them because I mistake know that God is the ultimate physician, so I just prayed for that Lord, let me be your hand, give me your wisdom, give me your mind and I prayed for that every day that I would not harm anybody that I would learn and I would take great care of them. 
P16: So I was excited at first, really really excited and then once I got the Terrified, job and realized that it was like jumping from the afire pan to a fire with undertrained, no training, I was absolutely terrified, like I was anxious ...my first anxious, worried whole year and a half was like I was so anxious and worried all the time, like I was not sleeping well at night, because I felt so unprepared...

Positive P1: So after graduation I felt very excited, nervous, and proud because I emotions just was happy that I advanced with my education and was able to care for patients, the way that I wanted to ... I kind of felt a sense of independence...

Excited, nervous, proud, happy due to completing school, achieving and educational goals, having an increase in autonomy of decision making

P3: ... after 1 year of no job and not finding a job, eventually I landed a Excited about job, super excited, I am super excited...

finding a job

P6: I think it was exciting, because I think a lot of people once you got to

Excited about go back to school ... I won't have to do this that much often you know leaving RN role some of the things in nursing that you kind of got tired of or burn out of, you knew your role was changing so that kind of feels that excitement as a new grad that you are going to be a new NP grad...

P8: ..a little bit of exciting because you are learning new things and you are getting a new job, and am having new experiences...

Excited about learning new things, gaining a new role, getting a new job

P9: Excited, I was ready to transition from being an RN to NP because I Excited about was not overly excited with some of my RN positions...

leaving $\mathrm{RN}$ role P12... it feels a little bit rewarding to get your first pay check you know

Rewarded and 
that was exciting, more paid...

P12: But also more rewarding because you are the one making the diagnosis, treatment, and you actually get to see ...them better and you know, you are responsible for that.

P14: ... I was excited when I finished school because it was like finally OMG, I am going to be like them, be like them and all the people who trained me and I can exceed that confidence and then rude awakening...

P15: ... at first relief, I finally made it through school and then stress as ...I had passed the board exam and looked for a job and take on the new role, but it was exciting too because to know that I accomplished the goal and that I was able to now make an impact on patients health more so than what I was able to do as an RN. excited due to pay

increase

Rewarded about

helping patients

Excited due to

finishing school

Relieved and excited due to finishing school, passing boards, and taking a new role with the possibility of making a greater impact

Note. Theme VII. Negative and positive emotions and reactions.

\section{Summary}

All participants in this study were interviewed using the interview protocol, which assisted in exploring of the research question. Participants reported multiple challenges during their clinical experiences while transitioning to their NP role, both before and after graduation from their NP programs. Their challenges were related to their preparation for clinical experiences, preceptorship process, learning opportunities, learning environments, and their change in autonomy and responsibilities. 
Data showed that NPs' role transition to practice was ill-supported through preceptorship before and after graduation. Data showed that when in school, the autonomy of decision making in a clinical environment was often not present. Due to scarce clinical hours, frequent site changes, and preceptors' workload, hands on learning was insufficient, encouraging students' dependency on their preceptors' judgment in making critical clinical decisions. To be effective, clinical experiences had to be conducted through the "hands-on" active learning when preceptors had a "carved out" time to teach their students.

Additionally, frequent preceptor changes led to a lack of preceptors' comfort with the students, which resulted in inadequate clinical training, leaving out important procedural and diagnosing skills. When transitioning to a real world practice, participants often lacked structured preceptorship. Their collaborating MDs were often unfamiliar with their educational needs and were available to supervise them but not to mentor. When advancing to practice after graduation, participants were learning their role from all setting's providers if available, regardless if they had a collaborative practice agreement with these providers or not. Many also reported not receiving the necessary support from their collaborative physicians, calling them "collaborators on paper." Difficulties in applying learned skills to new and complex clinical environments that were different from what participants encountered in school were also consistently reported.

While attending their NP program, most $(68.8 \%(\mathrm{n}=11))$ participants had a responsibility of finding and securing their clinical preceptors and clinical sites, which caused participants to "settle" on "whatever sites" they could find. Though significant challenges were reported by most participants in finding and securing clinical sites, inconsistencies in quality of clinical 
experiences were noted to be similar regardless of whether the sites were found by the schools or by the participants. More specifically, participants reported frequent changes of clinical sites and clinical preceptors and site selection was often based on sites' geography and availability. Not being familiar with their preceptors' expectations, participants often just "showed up day one" for their clinical experiences. Besides being unfamiliar with their preceptors' expectations prior the start of their clinical experiences, participants reported that as they started to learn their preceptors' styles and expectations, these varied significantly. Thus, some experiences were reported as being successful and some were noted to be less effective. Many reported that they had to build trust and comfort level of their preceptors' with their skills almost from "the ground up" with the start of each clinical rotation, which led to more "shadowing' time. Many reported having at least one clinical experience when shadowing was used as a main teaching strategy by the preceptor during the entire time of the clinical rotation, rendering it less effective.

The patient care opportunities also varied among the sites, making it a "random" experience; as a result, at some sites participants had sufficient quality and number of patient care opportunities to meet their learning needs, whereas at others they did not. Further, the “random" nature of participants' clinical experiences along with frequent site changes, led to the lack of procedural training, laboratory tests interpretation, coding, as well as lack of experiences with having to make more complex patient diagnoses. These inconsistencies caused participants graduate without being exposed to all the necessary clinical situations such as various procedures and complex clients. 
Many attributed their lack of diagnosing experience was due to the fact that their patients either already had establish diagnoses or the diagnoses that the participants had to make were straightforward and did not require a lot of critical thinking abilities. However, many also reported being at their clinical site for one or two days a week only as well as inability to follow up on the patients they had previously seen, which could have accounted for the lack of critical thinking and diagnosing experiences. Thus, lack of clinical hours and infrequent clinical days were found to negatively impact the participants' ability to gain necessary clinical knowledge.

Furthermore, participants' autonomy was never approximated to what they have experienced in transition to practice after graduation. They reported to often be "attached to the preceptor's hip" and not taking their responsibilities as "seriously" as they did after graduation. More specifically, frequent inability to either have a consistent EMR access or consistently document on their patients during their clinical experiences while in school, led to more reliance on their preceptors. Notably, after graduation many participants reported reliance on their EMR for documenting, evaluating patients' risks for drug-drug interactions, retrieving patient related information, and communicating with other team members. Data showed a mismatch of the inconsistent EMR use during the clinical experiences in school and heavy reliance on EMR after graduation. Furthermore, some misalignment between the didactic assignments and clinical experiences was reported to cause participants to rely more on their preceptors for guidance, decreasing autonomous decision making abilities. Moreover, participants often reported their preceptors being busy, which sometimes led to the lack of "hands on" clinical experiences, which again led to decrease in autonomy and increase in participants' reliance on their 
preceptors. In contrary, when preceptors had sufficient time to teach, participants' ability to learn by doing improved. Further, occasionally participants reported more hands on experiences when their preceptors were NPs; they attributed this to that their NP preceptors had more realistic expectations and grater trust in their abilities then their MD counterpart.

After graduation participants tried to embrace their role; but, found to be overwhelmed by unexpected lack of support and employers' expectations of them to function independently and to their full ability "right out of the gate." Participants reported that in their new role they would often take their work home and having to complete their duties of charting on their own time. Most were placed in "sink or swim" situations due to the lack of structured and consistent NP onboarding procedures. Participants reported that their onboarding frequently consisted of them shadowing providers in the office while waiting for their credentialing process to complete, which often lacked "hands on" training. However, when starting working more independently, many reported that slowly "ramping up" the number of patients on their schedules was effective in allowing participants to have sufficient time to learn while practicing more autonomously. Lack of consistency in participants' leaning experiences was reported by many. While several reported that some collaborators and providers were not willing to help them learn, many other providers were often willing to help; however, they were often busy with their own workload, which limited the amount of support participants could obtain during their transition. Whereas learning from multiple providers was found somewhat helpful in learning different work styles, it also caused inconsistencies and lack of familiarity when learning from multiple people as opposed to having one mentor to go to with questions. Further, some participants 
reported having no other providers at their first job; they reported having to learn alone while trying to master seeing full patient schedule day one of their new role. Participants reported to self-educate to fill the gap between their lack of training and the demands of their jobs using multiple resources. This caused participants to self-doubt themselves. Some were reporting missing their "safer" RN role; some were hoping to get into a specialty care to avoid a wider scope and high demands of primary care, while others doubted their decision to become an NP all together.

In addition, participants found that the reality of the real world practice was much more complex than that of their clinical experiences in school. Namely, they encountered many underserved patients with multiple comorbidities, polypharmacy, and unmet health needs that they were responsible for but undertrained. They were also undertrained in performing common office procedures, diagnosing complex cases, as well as interpreting laboratory tests. Such lack of training led to participants' scope of practice limitations while adjusting to the new role.

Data also showed that though many participants had RN background, they identified their role more with an advanced provider than with nursing. When missing the old and comfortable $\mathrm{RN}$ role, participants also missed the time when they were able to have sufficient time with their patients. Whereas they reported that they were able to transfer their RN skills of history taking, assessment, and building special relationships with their patients, the skills of diagnosing and treatment plan were new and were reported as medical skills.

In summary, participants graduated and realized that their learning clinical experiences at school did not approach the reality of clinical practice. They encountered limited support, some 
confusion about their role functions, and unrealistic employers' expectations. Despite the difficult role transition, all participants were functioning in their NP role at the time of the interviews.

In the following chapter five I restated this study purpose, summarized key findings, and interpreted the results in relation to the literature and theories discussed above. Additionally, I noted study limitations, provided recommendations for future research, as well as discussed this study implications for positive social change. 
Chapter 5: Discussion, Conclusions, and Recommendations

\section{Introduction}

The purpose of this hermeneutic phenomenological study was to understand and describe the meaning of NPs' role transition experiences while in a preceptorship before and after graduation. Many researchers have emphasized and studied post-graduation residency programs (AANP, 2017a; Flinter \& Hart, 2016). However, most novice NPs do not have an opportunity to attend such a program, with only a few NP residencies available across the country. It became important to study NPs' experiences with those transition support systems that are available to most novice NPs. Because NPs' role transition is a wide area, this research focused on a discrete component of transition related to preceptorship given that the literature review indicated that preceptorship needed restructuring.

I explored 16 NPs' experiences with their NP role transition and preceptorship, both before and after graduation, by conducting in-depth, face-to-face, Skype, and phone interviews. Novice NPs experienced significant challenges when transitioning to practice; an exploration of their experiences of learning the new skills in the context of their clinical environment, guided by their preceptors, helped explain how NPs' transition was supported through preceptorship. Data analysis using Van Manen's approach revealed seven major themes. Each theme included a preand post-graduation component.

The central finding of this study was that participants' fragmented and preceptordependent clinical experiences in NP school were followed by an abrupt increase in autonomy without adequate support when advancing to practice after graduation. I learned that when in NP 
school, participants were starting their clinical experiences without any pre-work to learn about their preceptor's expectations, the site's EMR and the site's workflow. When transitioning to practice after graduation, participants were often expected to fully function; they were either "thrown" into doing their NP job to "sink or swim" or they were given an opportunity to shadow other practice providers. Further, many reported that scarce clinical hours, overuse of shadowing, preceptors' unadjusted workload, and minimal procedural training led to the lack of learning opportunities and increased NPs' dependency on their preceptors. Data also showed that when transitioning to practice after graduation, participants attempted to learn their new skills from their busy collaborators, but most of the role learning was self-directed. Next, participants reported lack of complex patient care opportunities while attending their NP programs; however, they noted that after graduation they often had limited support and knowledge in caring for reallife, complex patients. Finding and securing clinical settings when in school was reported to be a major issue. After graduation, support provided to novice NPs was different for inpatient and outpatient settings. Working alongside other providers when transitioning to practice was more prominent in inpatient settings and was reported to be an important facilitator of participants' successful transition to practice. Finally, though many participants experienced feeling frustrated while looking for clinical sites at schools, they also reported feeling excited, nervous, and proud about learning new skills. However, after graduation, participants reported feeling often unprepared, overwhelmed, and afraid of making a mistake as they were experiencing unexpectedly steep, ill-supported, and sometimes lonely transition to practice. 


\section{Interpretation of the Findings}

This study confirmed that NP role transition is difficult and there is a sizable gap between NP education and practice that is ill supported. Similarly, NP role transition was reported difficult and stressful in the literature (AACN, 2015 Barnes, 2015a; Brown \& Olshansky, 1997; Flinter, \& Hart, 2016; MacLellan et al., 2015; Steiner et al., 2008). Fitzpatrick and Gripshover (2016) found that the difficult transition to practice may be related to the gap between the NP education and what is expected in practice. My findings also confirmed that the NPs' clinical training is ill-supported before (American Association of Colleges of Nursing (AACN), 2015; Donley et al., 2014; Logan et al., 2015; Roberts et al., 2017; Webb et al., 2015) and after graduation (Brown \& Olshansky, 1997; Flinter \& Hart, 2016; Steiner et al., 2008). Data further revealed the specific areas within the two role transition stages that need strengthening (Appendix E and F). The following is description of my findings in relation to the literature reviewed in Chapter 2.

\section{Theme I: Transition Preparation and Learning. Pre-graduation Meet and Greet Clinical Orientation to Post-Graduation "Winging it" Onboarding}

Assistance with participants' preparation to practice in the clinical environment was explored in relation to their role transition during the transition stages before and after graduation. Data showed that before the graduation except for the "meet and greet" orientation that some participants initiated prior their clinical experiences at school, there was no other preparatory work before the participants' start of their clinical rotation. More specifically, data showed that participants were not aware of their preceptors' expectations or work style until the 
start of their clinical rotations. Cusson and Strange (2008) underlined the importance of orientation during NPs' transition time. It is important for the preceptor to evaluate the trainee's knowledge and the level of independence (Babcock et al., 2014). Likewise, Nyhagen and Strøm (2016) and Rhrich et al., as cited in Poronsky (2012) stated that lack of understanding of each other's expectations forms a barrier to effective preceptorship. Flott and Linden (2016) noted that for the learning to take place, students must be familiar with the environment, feel safe in it, and be respected and recognized by the health care team. Participants reported that it was important for them and their preceptors to be comfortable with each other prior NP students seeing patients semi-independently. Preceptors were not familiar with NP students' clinical preparation level and required time to get to know their students' learning needs. Babcock et al. (2014) noted that training NPs should include preceptor's orientation to the trainee's course syllabus, objectives, and expectations.

Some disconnect between the didactic and clinical work was also reported. The lack of didactic knowledge as well as multiple didactic assignments not connected to participants' clinical experiences were reported to have a negative effect of participants' clinical performance. Similarly, Giddens et al. (2014) noted that preceptors expressed frustration about the variability of NP students' preparation level; preceptors carry the burden of having students who have not been checked for clinical knowledge in their didactic portion of the program. Data also showed that sometimes participants were used as an "extra set of hands" to perform RN level work at their clinical sites. It is possible that this regression in the role was caused by preceptors' discomfort or disappointment with participants' skill level. Brown and Olshansky (1998, p. 55) 
referred to this as "stepping back in expertise" and Roberts et al. (1997) called this regressing to the role of an RN. Solid foundational knowledge is an important prerequisite of clinical experience and should be taught prior students attending their clinical rotations.

Lack of EMR access affected participants' retrieval of patient related information, necessary for them to prepare for making patient care decisions during their clinical learning experiences. Roberts et al. (2017) noted that EMR access was one of the barriers to successful precepting of NP students. The AACN (2015) and Erikson et al. (2014) reported that 75\% of academia leaders were concerned with students' issues of accessing EMR. Providing students with EMR training prior the practicum start date decreases the number of clinical hours lost on such training during the practicum (Babcock et al., 2014). This study confirmed that EMR access was essential for NP students' patient care decision making process in a clinical environment. Extending current understanding, this study helped to make a connection between the lack of NPs' documentation experience prior to the graduation and significant reliance on EMR during their role transition after graduation. Data showed just how important it is for NP students to learn how to manage the electronically stored patient data. Not only was the EMR system important for the novice NPs' data retrieval after graduation, it also played an important role in NPs' management of patient visit time, which is required for NPs to stay employed.

Immediately after graduation, many participants reported feeling unprepared to practice independently. Similar to my findings, Hart and Macnee (2007) cited lack of preparation in most of the surveyed NPs. Logan et al. (2015) and Jones et al. (2015) stated that many NPs struggled with their clinical skill level preparation. However, adequate clinical preparation is essential for 
NPs' successful transition to practice (Clabo et al., 2015). This study showed that participants employer' expectations of their performance were unrealistic, affecting their transition to practice. Similarly, Fitzpatrick and Gripshover (2016) and O'Brien et al. (2009) noted that MDs expected NPs to function at an expert level right after graduation. Further, NPs' transition to practice was affected by the gap between the employers' expectations and newly graduated NPs' entry preparation level (Andregard \& Jangland, 2014; Sargent \& Olmedo, 2013). I found that while employers still expected novice NPs to fully function immediately after graduation, they often allowed for novice NPs' schedule to slowly build, gradually increasing the number of patients. Though “ramping up” novice NPs' schedules were reported as helpful, most still reported lack of structured onboarding process. This finding was significant because it showed that many employers were still unaware of the sizable gap between NP education and NP practice. Data showed that this unstructured onboarding often led to extensive self-teaching leaving participants to doubt their competence.

\section{Theme II: Preceptorship During Role Transition and Learning. Pre-graduation "Hands- off” Learning From Busy Preceptors to Post-graduation Self-Teaching}

I found that hands-on learning with consecutive clinical hours fostered more autonomous clinical decision-making skills. Similarly, Flott and Linden (2016) found that student's engagement and instructing strategies influence student's learning. My results showed that the most effective instructional strategy reported was "see one, do one," in which preceptor would demonstrate the skill and then observe the student perform the skill, after which, the student would perform more independently. 
Hands-off clinical experiences combined with "chopped" clinical hours encouraged participants' dependence on their preceptors with consequent decrease in quality of their clinical experiences. Many participants reported that some of their preceptors thought that hands off learning or shadowing were sufficient as they exercised this teaching strategy throughout the entire rotation. Preceptors' teaching styles were reported to be inconsistent. This finding is similar with Bazzell and Dain (2017) who reported that no preceptorship guidelines exist for those preceptors who train NPs. This lack of preceptors' training may have been responsible for such inconsistency in the quality of participants' clinical instructions.

Preceptors' workload and lack of day to day feedback frequently prevented participants from having adequate clinical experiences increasing their dependence on their preceptors during their clinical rotations at school. This was consistent with results from Marfell et al. (2017) who noted that preceptor's frequent feedback is essential for a trainee to progress to a greater degree of independence. In accordance with Hallas et al.' (2012) results, that showed that no evidence exists that 500 clinical hours translated into required NPs' competencies, my study showed that participants did not have enough clinical hours to train appropriately.

Because of lack of preceptorship prior to graduation, after graduation participants learned their NP role through self-teaching and asking questions. Results showed that participants rarely had an assigned preceptor to help them to transition. Irwin, Bliss, and Poole (2018) reported that preceptorship positively effects nurses' competence and confidence and that having a team of preceptors may be more efficacious than having a single preceptor assigned. However, lack of preceptorship to help with role transition was consistent with Marfell et al. (2017), Wilkes and 
Feldman (2017), and Hart and Bowen' (2016) who reported that formal preceptorship is uncommon with only $17 \%$ of novice NPs reported having a formal preceptor during their role transition at work. In this study $24 \%(n=4)$ of participants reported having a formal preceptor assigned. Though all participants had collaborative agreements with their MDs, they frequently reported that their collaborators were not the ones teaching them. Jones et al. (2015) too noted that with the lack of formal preceptorship to support novice NPs' role transition after graduation, the major source of preceptorship for NPs became through having a formal collaborative agreement with their assigned physicians. However, my results showed that MDs often lacked knowledge about participants' NP role and scope. Lipley (2014), Schadewaldt et al. (2013), Schadewaldt et al. (2013), and Van der Biezen et al. (2017) also noted that MDs were not as familiar with the NP role and scope of practice, which was a common barrier to a successful NP and MD collaboration. Data showed that NPs often expected MDs to mentor them; however, instead MDs were often reported to simply supervise NPs.

Participants' desired structured preceptorship, which was also consistent with Bazzell and Dains (2017) who stated that structured preceptorship had a positive effect on NPs' clinical reasoning. Because of such lack of preceptorship, participants were trying to learn from everyone in their practice; however, providers' workload was often described to be a barrier to participants' learning their NP skills. Though providers were often busy, novice NPs reported alternating those to whom they would go with questions to ensure they did not "bother" providers. This strategy was reported as helpful especially when participants had other NPs working alongside. This finding was consistent with Heitz et al. (2004) who described collegial 
and physicians' support and networking as important role transition facilitators. Well-structured onboarding process was determined to be a missing link in participants' transition to practice. Similarly, Hart and Bowen (2016) found that about $90 \%$ of NPs expressed an interest in postgraduation residency programs; however, in 2016, only 37 nurse practitioner primary care postgraduation training programs graduating approximately 150 NPs annually were available across the United States (M. Flinter, personal communication, June 8, 2016). This study confirmed the need for post-graduation structured preceptorship process.

Results revealed that, both before and after graduation, working with one preceptor was not participants' reality as they were frequently learning from whoever had the time. This finding was similar to AACN, (2015); Giddens et al., 2014; LeFlore, and Thomas (2016) who stated that one-on-one preceptorship model used for NPs' clinical education is outdated. Learning from other professionals is an important attribute of NPs' successful transition to practice (Faraz, 2016; Farrell et al., 2015). Data showed that there is a need in restructuring current state of preceptorship through incorporating the interdisciplinary learning. However, for that to be effective, professionals should better understand each other's' roles and functions.

Both before and after graduation, participants reported their preceptors' lack of time to teach them, which affected participants' ability to learn their clinical skills. This finding was consistent with Roberts et al. (2017) who found that the most frequently cited barrier to precepting was the amount of time it takes to precept. Bazzell and Dains, (2017), Forsberg et al. (2015), Giddens et al., (2014), Keough et al. (2015), Poronsky, 2012, and Roberts et al. (2017) noted that preceptors' priority is to take care of their patients within the strict time constraints of 
their schedules. This study showed that case discussions and debriefings were frequently omitted from students' learning experiences due to the time constrains. Overall, dependence on their preceptors prior the graduation was changed to participants' seeking support and guidance after graduation. When such support was not found, self-teaching was employed extensively by all participants.

\section{Theme III: Learning to Care for Complex Patients. Pre-graduation Random and}

\section{Fragmented Clinical Experiences to Post-Graduation Complexities of a Real Life}

Data showed that participants' experiences with learning complex patient care at school were random, limiting the quality of participants' clinical training. In accordance with my findings, LeFlore and Thomas (2016) stated that quality of learning suffers from great variability and random occurrence of patient care experiences among the sites. When advancing to clinical practice after graduation, participants reported "rude awakening" as they did not feel prepared to care for complex patients right after graduation. Data also showed that lack of organizational support significantly affected participants' transition experiences as they did not yet feel equipped to care for complex clients and were in need for such support. Jones et al., (2015) reported that novice NPs expressed having significant difficulties and lack of preparation in prescribing for elder adults with multiple comorbidities and multiple medications. Graue et al. (2015) reported about NPs' significant difficulties caring for patients with multiple comorbidities. Participants particularly struggled with polypharmacy, comorbidities, noncompliant clients, complex diagnoses, underserved, and less known patients. Flinter and Hart (2016) noted that the NPs' transition process from academia to practice after graduation was 
unpredictable and lacked support. Without having adequate structured support, participants struggled with managing their time as well as took some of their work home.

\section{Theme IV: Learning in Clinical Environment. Pre-Graduation "Begging" for Preceptors and Sites to Post-Graduation "Collaborator on Paper."}

Results of my study showed that before the graduation, most NPs' reported the greatest challenge was finding and securing clinical sites and preceptors, which often lead to mismatch between the preceptors, sites, and student's learning needs. Challenges of obtaining clinical sites and preceptors were well documented (AACN, 2015; Erikson et al., 2014; Drayton-Brooks et al., 2017). Literature showed that interests, schedules, and interaction styles need to be considered when choosing a preceptor (Marfell et al., 2017). A good match between a student, clinical site, and a preceptor may facilitate NP students' role transition (Heitz et al., 2004). Mismatch between the clinical sites and students' learning needs in this study could lead to limited training opportunities available to students. Evidence showed that $38 \%$ of NP program representatives expressed concerns with an inadequate number of medical conditions students are exposed to through their clinical education (Erikson et al., 2014). If the mismatch between the preceptor and the student occurs, both student and school can suffer (Forsberg et al., 2015). Flott (2016) reported that physical space and communication was important for participants' successful role transition. Heitz et al. (2004) found that lack of adequate clinical sites was a significant role transition barrier. In this study, though finding clinical sites was a prominent issue for most, five out of 16 participants had their schools find clinical sites for them and their experiences with role transition after graduation were similar to those who had to find own clinical sites. Whether the 
sites are chosen by the students or by schools, student-site match may still be inadequate. This study extended current knowledge showing that even though securing clinical sites is a significant issue, the culprit of difficult transition may lay in what happens at the site after clinical start.

While locating the sites was one of the most stressful components of participants' experiences, participants also reported frequent preceptor change with limited number of clinical time spent with each preceptor leading to inadequate preceptorship. Quality of learning suffers from great variability and random occurrence of patient care experiences among the sites (LeFlore, \& Thomas, 2016). Heitz et al. (2004) and Steiner et al. (2008) found that during NPs' role transition in academia, curricular issues of inadequate preceptorship and lack of clinical time exist. NP students who spend at least three months with one preceptor develop higher selfefficacy levels (Neal, 2008 as cited in Poronsky, 2012). Placing students in one clinical environment for the entire length of their experience may be beneficial in students' understanding of the health care system and developing an effective preceptor-student relationship (AACN, 2015). NP students' fragmented experiences with clinical rotations make students' experiences less meaningful placing an additional burden on clinical sites (Giddens et al., 2014). In this study, short clinical rotations did not allow for the participants or their preceptors to become comfortable enough to allow students to perform more autonomously. As stated earlier, participants had limited opportunities to become familiar with the preceptors and sites prior the start of their clinical rotations. Flott and Linden (2016) emphasized that NPs' familiarity with the site or a clinic is significant for successful learning of a new role. This study 
data showed that in addition to students' familiarity with preceptors and sites, preceptors' familiarity and comfort with students must also be present before preceptors allow NP students to perform any hands-on training on their patients. This finding was not found in the previous literature review. Data showed that preceptors' comfort with students' performance was necessary before any hands-on training was initiated, extending students' shadowing experiences. Because the observation or shadowing time was not accounted for, in participants' clinical experiences, their hands-on clinical experiences were shortened. The combination of frequent preceptor changes with increased shadowing times amplified students' overreliance on their preceptors' decisions, decreasing their learning of making decisions autonomously. After graduation, this overreliance abruptly changed to a complete autonomy, which caused participants to feel unprepared to make their own clinical decisions.

After graduation, participants' relationship with providers they were learning from was reported as important to their successful transition to practice. Those participants who were learning alone reported more challenging transition experiences. Besides having more limited support, those who were the only NP in practice struggled more with the unrealistic expectations of their employers. However, those who were working alongside with other NPs reported a less challenging transition experience. Similarly, literature showed that face-to-face communication was found to be imperative in creating a good collaborative relationship between NPs and MDs (Schadewaldt et al., 2013). Nurses and physicians collaborate better in high acuity smaller areas (O'Brien et al., 2009). Heitz et al. (2004) pointed out the support by the colleagues and collaborative MDs as extrinsic transition facilitator during the role transition after graduation. 
Though having a formal preceptor was reported to be rare as reported in the literature (Wilkes \& Feldman, 2017), many reported having an informal preceptor or mentor they could go with questions. Frequently, participants were learning from all or any providers working alongside. Learning from other professionals is an important attribute of NPs' successful transition to practice (Faraz, 2016; Farrell et al., 2015). Participants expressed the need for structured preceptorship saying that they were looking for one but never found it. My results echoed Hart and Bowen (2016) and Jones et al.' (2015) findings that most NPs expressed the desire for such support by indicating the need for post-graduate residency programs to assist with their transition.

Though all participants had an assigned collaborative physician, many reported that this relationship was more of a formality as their collaborators were unfamiliar with their learning needs, often providing participants with little guidance. This was consistent with Lipley' (2014) statement that misunderstandings about the NP role and scope of practice appear to be a barrier to their role acceptance. When attempting to communicate with their collaborators, several participants reported difficulties reaching or engaging them when they needed help. In some instances, collaborators were not present on site leaving novice NPs to struggle with the full load of patient assignments. Being left alone to learn and master their new skills generated feelings of stress and fear in the participants. 
Theme V: Transitioning to a Greater Autonomy and new Responsibilities. Pre-Graduation “Attached to the Preceptor's Hip" to Post-Graduation “Jack of all Trades."

Participants' experiences with their new responsibilities and increased autonomy during their role transition before and after graduation were explored. I found that increased dependency on preceptors along with decreased hands-on clinical experiences led to reduced autonomy during the school clinical experiences. Many participants expressed that they did not recognize the full weight of their new responsibilities until starting their new jobs. Similarly, Cusson and Strange (2008) noted that the time NPs start performing independently or semi-independently may mark the time when most NPs recognize the need to assume additional responsibilities. When in school, participants reported little independent decision making, prescribing, or practicing differential diagnoses in clinical practice.

Data showed that after graduation, real life complexities of patient care and sudden increase in autonomy, accompanied by the lack of support and lack of others' familiarity with the NP role, led to participants' feeling unprepared and fearful. Most skills are developed when NP starts his or her new job (Bazzell \& Dains, 2017). Forty seven\% of NPs felt that they were practicing outside their scope of practice during their first year of practice (Hart \& Bowen, 2016). What my study showed was that the lack of autonomous decision making skill acquisition practice when in school may be one of the causes of the academia and practice gap.

While participants were prepared to practice at the beginner level of their role after graduation, the complexities of patient care, which required a higher level of expertise, along with the expectations of others for NPs to practice autonomously, created a more difficult 
atmosphere of transition for the participants to overcome. Fitzpatrick and Gripshover, (2016) and O'Brien et al. (2009) noted that MDs expected NPs to function at an expert level right after graduation. NPs' transition to practice is affected by the gap between the employers' expectations and newly graduated NPs' entry preparation level (Andregard \& Jangland, 2014; Sargent \& Olmedo, 2013). NP dependency on his or her collaborator physician right after graduation may have created an unintended conflict based on unmet and unrealistic physician's expectations and their lack of knowledge about NPs' learning needs after graduation.

While data showed that participants were overwhelmed with their new responsibilities, they also appreciated the new autonomy level and began to embrace it when becoming more comfortable with the new responsibilities. Similarly, Schadewaldt et al. (2013) noted that whereas many NPs seek a collaborative relationship with MDs, many would also like their autonomy of practice to be respected. Though working in a team is essential, it is also important to consider role boundaries and each role-specific function, for the collaboration to be successful (MacNaughton et al., 2013). Data revealed that though NPs longed for a strong and structured preceptorship from their collaborators, they also liked their autonomy. Defining role boundaries can facilitate successful collaboration among the different professionals through considering each professional's workload, physical space, leadership, education, as well as individual's attitudes and values (MacNaughton et al., 2013). Results showed significant variation regarding the establishment of boundaries when in practice, with some participants functioning completely independently and some sharing their workload with other providers. 
After graduation, participants also reported having particular difficulties with autonomously prescribing multiple medications, coding, differential diagnoses, and choosing and interpreting the laboratory tests. Documentation, billing, and coding were identified as weaker areas during the NPs' transition (Dillon, 2016). Likewise, Jones et al. (2015) noted that novice NPs expressed having significant difficulties and lack of preparation in prescribing for elder adults with multiple comorbidities and multiple medications as well as lack of training to perform procedures, such as suturing, fracture care, X-Ray readings, etc. (Jones et al., 2015). In addition to struggling initially with their new responsibilities, participants often reported being rushed. More diagnostic errors are done by those who use the least time to complete patient visits (Pirret, 2016).

\section{Theme VI: Embracing the Role and Identity Confusion. Change From the "Notify the Provider Mindset”}

Results revealed that prior to graduation, practicing in an $\mathrm{RN}$ role was helpful for some and confusing for others. Being in a role of NP student and RN was described as "practicing in both worlds." These participants reported they tried to keep their knowledge to themselves to avoid conflicts with treating providers when working in an RN role. Similar to my results, Jangland et al. (2016) noted that being positioned between the two roles may cause identity confusion. Though Steiner et al. (2008) found that prior life experiences may facilitate NPs' role transition; Barnes (2015) was not able to detect the significant relationship between previous RN experience and NPs' role transition. Barnes (2015b) and Poronsky (2013) noted that to identify with the new role, it may be necessary for an NP student or a novice NP to separate from an old 
RN role to focus on the transition on a new role. Heintz (2004) reported that NP students' role confusion and role separation factors influenced NPs' role transition. In accordance with current research, mythis study showed conflicting results on whether practicing in an $\mathrm{RN}$ role was helpful for participants' role transition prior the graduation.

Participants described that their role change after graduation started with excitement as they welcomed and embraced the new role, but quickly changed to the realization of new responsibilities of a new role combined with feeling unprepared to care for complex patients and the lack of transition support. Similarly, Barnes, 2015b; Chang, Mu, and Tsay (2006) noted that as NPs change from taking orders from physicians to taking over the care of a patient and giving orders, there is a shift to a greater autonomy and increased responsibilities in an NP's new role; this abrupt shift in NPs' mindset may be responsible for the reported identity confusion. Becoming a "prescriber of care" is an important attribute of NPs' transition to practice process (Barnes, 2015b, p. 140; Cusson, \& Strange 2008). Brown and Olshansky (1997) reported that perception of being an imposter while struggling to reconcile the role of a nurse and the provider can lead to self-doubt and high levels of stress in a novice NP. This study contributed to further understanding of NPs' role identity by providing a greater detail on how abrupt this mind set change was after graduation.

Participants expressed some difficulties with going back and forth between the two roles of an RN and NP. Data showed that $18.8 \%(n=3)$ of participants still worked in a role of an $\mathrm{RN}$ while concurrently performing in a role of an NP. Whereas many stated that after a while they started enjoying the autonomy of their new role, many reported day dreaming of returning to 
their "safer" RN role; however, too much was already invested in NP degree. It was also reported hard to not perform "nursy" duties that the participants were accustomed to and that others expected from them. Such unclear role boundaries led to confusion about the scope and functionalities of the NP role. Andregard and Jangland (2014) stated that the NPs' role appears to be blended into medicine and nursing creating confusion and friction on both sides of this professional spectrum. This study confirmed current knowledge that the boundaries between the nursing, advanced nursing, and medical profession are blurred effecting novice NPs identifying with their new role during their transition to practice.

While most participants were transitioning from an RN role, two NPs transitioned from other roles. These participants reported identifying themselves with the role of an advanced provider as opposed to identifying with the nursing role. This finding was important because non-RN to NP role transition may be different and may require different types of support. However, there is a paucity of literature that describes such experiences with NP transition. Theme VII: Transition Reactions. Changing from "Excited" to "Scared and Unfulfilled."

After graduation, among the 15 identified emotions participants experienced during their role transition, 12 were negative. Similarly, Barnes (2015b) noted that among the 11 emotions identified in transitioning of NPs, ten were reported as negative. These emotions often included feelings of "fear, insecurity, being overwhelmed, inadequate, vulnerable, and isolated" (Heitz et al., 2004, p.417) and "feelings of chaos, turmoil, and confusion” (Brown \& Oshlansky, 1997; MacLellan et al., 2015, p. 394). This study results showed that participants felt fearful of making a mistake that could negatively affect their patients' health. NPs' feelings of self-doubt and 
incompetence as well as mixed negative feelings were caused by their expanded responsibilities (Hart \& Macnee, 2007; Heitz et al., 2004). Horner (2017) noted that NPs' job satisfaction was closely connected to the NPs' ability to meet increased responsibilities and their sense of autonomy. My results showed that participants often found themselves frustrated with the abrupt increase in their autonomy after graduation because of the inability to practice independently and having to rely on others for additional knowledge. Though many specifically looked for a structured preceptorship seeking help with their new responsibilities after graduation, they realized that this type of support rarely exists.

Negative emotions to the difficult process of NPs' transition could have contributed to NPs' retention in this study, as many participants reported having more than one NP job postgraduation. Similarly, literature showed that more than a quarter of novice NPs will leave their job within one year (American Association of Colleges of Nursing, [AACN], 2014). This study contributed to further understanding of how novice NPs feel when transitioning to their new role. It was important to explore this part of participants' experience because human feelings and reactions constitute an important part of any experience.

\section{Theoretical Interpretation of the Findings}

The theoretical lens for this study consisted of the combination of two theories, the Schlossberg's Transition Theory, and the Collins et al. (1991) Cognitive Apprenticeship Model (CAM). The concept of transition was guided by the transition theory, and the concept of preceptorship was guided by the CAM. 
According to CAM, the elements of cognitive apprenticeship are important for the effectiveness of the training process. However, only the elements of traditional apprenticeship were found to be present during this study data analysis and the components of cognitive apprenticeship were rarely described by participants. Whereas both traditional and cognitive apprenticeships involve teaching methods of modeling, coaching and scaffolding, cognitive apprenticeship also includes articulation, reflection, and exploration (Figure 1; Collins \& Kapur, 2015). Data showed that modeling teaching technique was utilized extensively through allowing participants to observe their preceptors. Often coaching was also reported, in which preceptors observed and guided NPs in their performance. Furthermore, scaffolding was frequently reported when participants described their preceptors helping them to diagnose and treat their patients. However, while CAM guides to slowly decrease such supports to increases students' autonomy, data showed that this was rarely done as most of the time they were "attached to the preceptor's hip." Articulation teaching method was rarely commented on. Though some participants noted that their preceptors used questioning to allow them articulate their strategies, preceptors were often too busy to employ this strategy. Next, reflection as a teaching method was almost never reported. Participants rarely had a chance to reflect and debrief with their preceptors on their patients at the end of the day to understand how their strategies compare to those of their preceptors and practice standards. Because day to day feedback was uncommon in participants' training, they had little opportunity to reflect on what could have been done differently with their preceptors on a regular basis. Lastly, exploration teaching strategy was reported to be applied inconsistently. While preceptors advised students to explore the guidelines related to the 
patients' issues, participants were limited in how much time they had for this "extra" work. No consistent follow up on these explorations was reported. As a result, data showed that most of the time participants were learning through the traditional apprenticeship as opposed to the cognitive experiential learning, which limited participants' learning of their new skills.

When compared to the traditional apprenticeship, in a cognitive transfer of knowledge master ensures apprentices' understanding of the process of a given skill, adjusting apprentices' experiences to their theoretical knowledge to ensure the relevance of their experiences, and encouraging the apprentices' ability to transfer common tasks across the different contexts (Collins et al., 1991). Though both traditional and cognitive apprenticeship involve learning skills in a specific context, in cognitive apprenticeship the teachers' and students' cognitive process is brought to clear view of both (Collins \& Kapur, 2015). Another distinction between the two includes that in cognitive apprenticeship students' tasks are chosen based on students' learning needs as opposed to be context dependent only (Collins \& Kapur, 2015). Most participants reported that their learning opportunities were site and preceptor dependent but rarely based on their leaning needs. Participants reported that their patient care opportunities were dependent on their preceptors' comfort level with their performance and were random.

According to CAM, content or baseline knowledge is essential for students' experiential learning (Collins \& Kapur, 2015). However, participants reported occasional disconnect between their didactic and clinical courses when they did not have a chance to cover yet what they have been experiencing in their clinicals. Many reported lacking the knowledge of how to diagnose, interpret laboratory tests, and perform procedures prior to starting their clinical experiences. 
Similarly, after graduation, though participants reported having good interviewing and assessment skills, they reported lack of deeper knowledge including complex diagnosing skills, procedural skills, and laboratory tests' interpretation skills. Learning these skills "on the fly" was reported stressful as others expected them to have this baseline knowledge prior to taking the job. CAM guides to the use of sequencing in experiential learning of students. Learning "global before local skills" is an important strategy; "apprentices learn to put together a garment from precut pieces before learning to cut out the pieces themselves" (Collins \& Kapur, 2015, p.6). Following this model principle, observation was frequently reported in this study to allow students to grasp the general process of a patient visit. Shadowing was frequently required prior participants being able to start performing more specific functions such as collecting patient history and assessing. In addition to learning general before specific, sequencing also implies that students' learning opportunities will be slowly increased in complexity and diversity (Collins \& Kapur, 2015). This was rarely reported in this study as NPs stated that their patient care opportunities depended on what was available to them, "you get what you get." After graduation, participants reported an abrupt increase in complexity of their patients, which they felt unprepared to manage. During this time, many reported that their organization would slowly increase the number of patients on their schedules to allow learning to take place. Though this was an effective strategy, lack of other structured onboarding procedures led to participants having difficulties with addressing patients' complexities. The reported lack of alignment between the didactic course and participants' clinical rotations in school along with inconsistencies among the clinical sites made it more difficult for the participants to acquire new 
knowledge. Similarly, after graduation little structure was provided to gradually increase the complexity and diversity of participants' experiences.

Next, CAM guides to using the sociology principle in supporting experiential learning through the use of learning environment. While in the program, many reported struggling with finding their clinical sites. The appropriateness of clinical learning environments was rarely known by participants until after the clinical start day. After graduation, participants often reported the lack of match between the clinical settings they learned in at school and the setting where they found an employment. According to the CAM, cognitive experiential learning allows one to utilize knowledge learned in one setting across other settings (Collins \& Kapur, 2015). Many participants expressed difficulties with applying the information learned in clinicals to their job setting. It is possible that not incorporating cognitive model into the NP training could be one of the reasons why NPs' transition to practice was difficult.

Schlossberg's transition theory guided the exploration of participants' transition stages and transition support. Schlossberg's transition theory indicated that the type and stage of one's transition, as well as available resources and one's coping mechanisms, can facilitate or inhibit successful transition outcomes (Anderson et al., 2011; Schlossberg, 2011). Both transition stages, before and after graduation were explored. As guided by the Schlossberg's transition theory, four transition support systems including the four "S's," situation, self, support, and strategies (Goodman et al., 2006) were examined during the two transition stages of participants' role transition. 
Data showed that in accordance with the transition theory assumptions, the available preceptorship support before and after graduation impacted participants' role transition experiences. According to the Schlossberg transition theory, the availability of resources may help determine if one's transition will be successful (Schlossberg, 2011). More specifically, the preceptorship support systems available prior the graduation significantly impacted NPs' transitioning to their new role after graduation. This study confirmed that it was essential to explore both transition stages (before and after graduation) together to holistically examine the attributes of participants' transition and their interrelationships with the transition stages.

\section{Limitations of the Study}

While I used sound strategies to produce quality results, my study had some limitations. One limitation was related to the accuracy of my data in that it depended on my participants' recall. The validity of the qualitative research is dependent on the quality of the information that the participants can provide about the research question (Patton, 2015). Because all participants have already graduated and started working in their new role, it was more difficult for them to recall their pre-graduation experiences. To increase validity or credibility of my data, I asked my participants prompt questions during the interviews along with directing them to describe their experiences with examples as opposed to opinions. I often restated participants' descriptions to ensure my understanding and correct interpretation of what was said. I encouraged my participants to provide thick description. I used interview data and field notes to triangulate data. After data collection, I had to exclude one participant when I discovered that she graduated from NP school outside the United States. To ensure that all my other participants graduated and work 
in the United States, I clarified this with all of my participants after the data collection. Data saturation was achieved at sixteen participants. Furthermore, I used debriefing along with the follow-up e-mails as needed after each interview to ensure the accuracy with my participants.

I included the two participants who had no prior RN experience before graduating with NP degree. Though including participants with no prior RN experience is not something I expected in this study, their experiences proved to be similar to those who had prior RN experience. The results showed that the demographic scenario of advancing to an NP role directly from other roles is more common in nursing than I thought. While the point of this study was looking at the role transition to a new NP role, future research can be done on the transition to NP role from roles other than nursing.

It was important to clarify possible bias. Potential bias can distort the reliability of my data. To avoid this, I used reflexivity describing my role as a researcher in this study to show how my personal experience and literature review can affect my results. In addition, I used journaling, filed notes, and transcripts to stay true to my study data. Notably, recruitment was limited to those participants who volunteered their participation. It is possible that NPs' experiences with transition could steer them in their decision to whether to participate in my study.

To increase my data dependability, I transcribed data myself and double-checked it for any errors and against my codebook. While I planned on using Skype to conduct face-to-face interviews to be able to include my observations into my data, many interviews were phone interviews instead due to participants' convenience aspect. While it may be possible to transfer 
the results of this study within the same population, transferring the results outside the study setting and the population is not possible unless additional studies are conducted. In qualitative research, internal generalization is possible; however, the external generalization is problematic (Maxwell, 2013). Though generalization of this study results would not be possible due to the nature of this study being qualitative and a small sample size, participants were distributed over a large geographic area of the United States including nine states.

Overall, the main limitation of this study was managing the large amount of data. I attempted to limit the amount of data in my study to make my study manageable using the interview protocol and by incorporating theories to provide for a theoretical lens to focus my study. The interview protocol helped me to limit my data to relevant information by focusing the interviews; it also allowed me to remember to cover all required study areas consistently across all participants. While I took care of pre-structuring my study to make it feasible and high quality, I also stayed open to the new emergent data.

\section{Recommendations}

The findings from this study extended the current knowledge on how people transition to the role of a nurse practitioner and how current state of preceptorship supports this transition process. The key findings from this study, point toward the insufficient support of NPs through preceptorship when in school and after graduation. More specifically, fragmented, poorly supported, inconsistent, and somewhat hands-off clinical experiences in school impacted NPs' autonomy during learning, which translated into an abrupt and poorly supported transition experiences after graduation. More research is necessary to determine the causative relationships 
among the factors that may be affecting NPs' transition to practice. It is important to note that despite of the difficulties, all participants demonstrated significant resilience and reported to be gainfully employed during the interviews.

This study had two participants who transitioned to an NP role from a non-RN role. Little is known whether having an RN background should be necessary for one to advance to an NP role. Few or no studies have been conducted on NP transition from a non-RN role. However, additional research on non-RN to NP role transition should expand current understanding of NP role transition.

This study included various NP specialty roles and settings, including inpatient and outpatient work environments. This study data showed that role transition was easier in an inpatient settings, despite the fact that NPs were trained to function in the outpatient settings. Additional research should focus on NPs' transition experiences for different NP specialties and settings in relation to the NP programs to gain a better understanding of how NPs transition in different settings and specialties.

This study did not find any variations in quality of school clinical experiences between participants who were responsible for finding their clinical sites from those, whose sites were found by school. More research may be needed to compare students' experiences and the methods for finding clinical sites.

Data showed that lack of interdisciplinary learning during NP students attending NP program may have impacted NPs' role socialization after graduation. Future research should 
explore the correlation between the role transition outcomes and various educational structures that involve specific disciplines to educate NP students.

\section{Implications}

Understanding how NPs' role transition is supported through preceptorship should guide future efforts directed at improving transition support for NPs. I addressed the gap in literature related to NPs' role transition and its support through preceptorship. Strategies to facilitate NPs' role transition can be developed and translated into practice based on this study's results. Social change can be affected by this study through the dissemination of this study's findings among the NP educators, NP employers, policy makers, and NPs themselves. The potential impact of this study results on positive social change is at individual, organizational, health policy, and practice levels.

\section{Implications for Organizations and Physicians That Hire NPs}

While the MD-NP collaborative agreement restricts NP's scope of practice to ensure safe and effective patient care, the evidence is lacking to support the effectiveness of such collaboration. While collaborative agreements emphasize the importance of physicians' supervision of NPs, the preceptorship component is missing in such agreements. Supervising alone may not provide NPs with the necessary structured onboarding. Interdisciplinary effort between physicians and NPs may be necessary to collaborate together with the goal of training rather than supervising alone.

The results of this study showed that the onboarding process for novice NPs can be improved. This study's findings offered useful insight on how to restructure preceptorship to 
facilitate NPs' successful transition to practice. The results showed that NPs' onboarding process lacked structure; participants' employers were reported to lack adequate knowledge and understanding of NPs' role. Participants reported that their employers expected them to function at their full capacity upon the graduation. However, they were prepared to function at the entry level. Creating a structural onboarding process for NPs when they enter employment is essential for improving NPs' role transition process, which may lead to increased NPs' retention rates, improved job satisfaction, and competence. More specifically, having a dedicated provider responsible for mentoring a novice NP along with the organization's specific structural program in place, may prove to be helpful. Based on this study results, it is advised that such mentor should have his or her workload adjusted to allow time for training the onboarding NP.

\section{Implications for NP Educators}

An additional social impact of this study results is at the academia level. This study data included the first transition stage that took place prior NPs' graduation from NP program. Results showed that the opportunities for participants' autonomy in clinical practice while in the program were insufficient. Frequent change in preceptors and sites as well as insufficient number of clinical hours did not allow for adequate patient care opportunities and necessary hands-on experiences. With each rotation, preceptors and students needed shadowing time to develop trust and comfort. Though observational experiences were necessary, they limited the number of hands-on opportunities, which led to inadequate learning of procedures and laboratory tests interpretation. Fragmented clinical schedules led to participants' inability to follow up on their patients, which led to decreased opportunities for practicing the diagnostic skills. Being 
"attached to preceptor's hip" and overreliance on preceptors' judgment led to participants' diminished autonomy of decision making during attending the NP program. This study offered additional insights on how NP programs can be changed to better prepare NP graduates for the complexities of a real world. Increasing the clinical hours and decreasing the number of clinical sites and preceptors may help in improving one aspect of NPs' clinical education.

Data also showed that preceptor's feedback to an NP student is insufficient. Additionally, study results showed that often preceptors did not have any "carved out time" to teach NP students, which frequently resulted in using hands-off teaching strategies. Notably for this sample, whether the clinical sites were chosen by a student or by the school, transition to practice was just as difficult. While more research is needed to establish the correlation between the clinical experiences and transition outcomes, this study results pointed toward that the issue is not only with finding and securing preceptors and sites, but it is also with what happens after student starts their clinical rotation experience.

\section{Implications to Nursing Profession}

The contribution of this study's findings to knowledge and nursing discipline can be appreciated with the realization of the significance of role transition support in the nursing profession. Filling the gap in the existing literature along with uncovering new elements of NPs' role transition and its support may help raise awareness of NPs' experiences with the role transition; it may help create a new scholarly dialogue that can lead to improved transition practices. Because research is limited in NP transition and preceptorship, this study's findings 
may prompt other scholars to conduct further exploration of this important phenomenon, which can lead to further improvements within NP profession.

\section{Conclusion}

NP role transition to practice is difficult and ill-supported. Preceptorship provided to support NPs' clinical skill acquisition is outdated and ineffective. This hermeneutic phenomenological study was conducted to understand and describe the meaning of NPs' role transition experiences while in a preceptorship before and after graduation. Four NP role transition attributes along with four preceptorship related concepts were incorporated into the seven interview protocol questions to explore novice NP' role transition experiences. Only recent NP program graduates were included in this study to gain information on the state of the most recent role transition experiences.

NPs' were found to have a difficult transition to practice, both before and after graduation from an NP program. Outdated preceptorship model used in NP schools was insufficient to provide NPs with necessary clinical knowledge. Upon entering their employment sites, NPs encountered unstructured onboarding process, which often lacked preceptorship. Though difficult transition was often reported during my interviews, all 16 NPs reported to be gainfully employed in their NP role at the time of the interviews.

Future research should focus on NP program improvements and structuring the onboarding process for NPs to facilitate the role transition after graduation. Recommendations for practice include improving preceptorship structure within the NP programs. Incorporating observation only clinical time while teaching NP students didactic courses may facilitate their 
adjusting to the new clinical environment and preceptors, while also allowing preceptors develop comfort level and learn about students' learning needs. To be helpful, the number of clinical hours must be increased significantly to allow for "blocks" of clinical experience as opposed to scattered clinical days. Further, I recommend starting "hands on" clinical rotations after the didactic portion of the program is completed, to allow sufficient time for extensive case studies review, procedural training, documentation training, and diagnostic skills development. After graduation, I recommend that a mandatory and setting specific preceptorship program be offered by all organizations that hire NPs. 


\section{References}

Ajjawi, R., \& Higgs, J. (2007). Using hermeneutic phenomenology to investigate how experienced practitioners learn to communicate clinical reasoning. The Qualitative Report, 12(4), 612-638. Retrieved from http://nsuworks.nova.edu/tqr/vol12/iss4/6

American Medical Association (n.d.). Guidelines for integrated practice of physician and nurse practitioner. H-160.950. Retrieved from https://www.amaassn.org/resources/doc/PolicyFinder/

American Association of Colleges of Nursing (2015). White paper: Current state of APRN clinical education. Retrieved from http://www.aacnnursing.org/Portals/42/News/WhitePapers/APRN-Clinical-Education.pdf?ver=2017-10-18-151801-160

American Association of Colleges of Nursing (2016). Nurse Residency program. Retrieved from www.aacn.nche.edu/education-resources/nurse-residency-program

American Association of Nurse Practitioners (2017a). NP fact sheet. Retrieved from https://www.aanp.org/all-about-nps/historical-timeline\#2000-s

American Association of Nurse Practitioners (2017b). Education. Retrieved from https://www.aanp.org/education/61-education/faq-np-prep/306-how-many-np-programsare-there

American Association of Nurse Practitioners (2017c). State practice environment. Retrieved from https://www.aanp.org/legislation-regulation/state-legislation/state-practiceenvironment/66-legislation-regulation/state-practice-environment/1380-state-practice-bytype 
American Association of Colleges of Osteopathic Medicine (2016). Matching self to program:

Residency programs. Retrieved from www.aacom.org/become-a-doctor/medstudents/career-planning/self-to-program/residencies

American Society of Health-System Pharmacists (2016). Residency resource center. Retrieved from www.ashp.org/rtp

American Physical Therapy Association (2016). American Board Physical Therapy Residency and Fellowship Education. Retrieved from www.abptrfe.org/RFPTCAS/GeneralInformationPrograms/

Anderson, M. L., Coodman, J., \& Schlossberg, N. K. (2011). Counseling adults in transition: Linking Schlossberg's theory with practice in a diverse world (4th ed.). New York, NY: Springe

Andregard, A. C., \& Jangland, E. (2014). The tortuous journey of introducing the nurse practitioner as a new member of the healthcare team: A meta-synthesis. Scandinavian Journal of Caring Sciences, 29(1), 3-14. doi:10.1111/scs.12120

Apprentice (2017). In Merriam-Webster's collegiate dictionary. Retrieved from https://www.merriam-webster.com/dictionary/apprentice

Arcury, T. A., \& Quandt, S. A. (1999). Participant recruitment for qualitative research: A sitebased approach to community research in complex societies. Human Organization, 128133. doi:10.17730/humo.58.2.t5g838w7u1761868

Babcock, P., Rosebrock, R., \& Snow, B. (2014). Tips for mentoring advanced practice nursing students. AACN Advanced Critical Care, 25(4), 322-325. doi: 


\subsection{7/NCI.0000000000000055}

Bargagliotti, L. A., \& Davenport, D. (2017). Entrustables and entrustment: through the looking glass at the clinical making of a nurse practitioner. The Journal for Nurse Practitioners, 13(8), e367-e374. doi:10.1016/j.nurpra.2017.05.018

Barnes, H. (2015a). Exploring the factors that influence nurse practitioner role transition. The Journal for Nurse Practitioners, 11(2), 178-183. doi:10.1016/j.nurpra.2014.11.004

Barnes, H. (2015b). Nurse practitioner role transition: A concept analysis. Nursing Forum, 50(3), 137-146. doi:10.1111/nuf.12078

Bazzell, A. F., \& Dains, J. E. (2017). Supporting nurse practitioner preceptor development. The Journal for Nurse Practitioners, 13(8), e375-e382. doi:10.1016/j.nurpra.2017.04.013

Baxter, P., \& Jack, S. (2008). Qualitative case study methodology: Study design and implementation for novice researchers. The Qualitative Report, 13(4), 544-559. Retrieved from http://nsuworks.nova.edu/cgi/viewcontent.cgi?article=1573\&context=tqr

Benner, P., Sutphen, M., Leonard-Kahn, V., \& Day, L. (2008). Formation and everyday ethical comportment. American Journal of Critical Care, 17(5), 473-476. Retrieved from file://C:/Users/vples/Downloads/Formation_and_everyday_ethical_comportment.pdf

Brown, M. A., \& Olshansky, E. F. (1997). From limbo to legitimacy: A theoretical model of the transition to the primary care nurse practitioner role. Nursing research, 46(1), 46-51. Doi: 10.1097/00006199-199701000-00008

Browne, K. (2005). Snowball sampling: using social networks to research non-heterosexual women. International journal of social research methodology, 8(1), 47-60. 
doi:10.1080/1364557032000081663

Brykczynski, K. (2012). Clarifying, affirming, and preserving the nurse in nurse practitioner education and practice. Journal of the American Academy of Nurse Practitioners, 24(9), 554-564. doi:10.1111/j.1745-7599.2012.00738.x

Budd, G. M., Wolf, A., \& Haas, R. E. (2015). Addressing the primary care workforce: A study of nurse practitioner students' plans after graduation. Journal of Nursing Education 54(3) 130-136.doi: 10.3928/01484834-20150218-21

Chang, W., Mu, P., \& Tsay, S. (2006). The experience of role transition in acute care nurse practitioners in Taiwan under the collaborative practice model. Journal of Nursing Research, 14(2), 83-92. doi: 10.1097/01.JNR.0000387566.34318.b2

Chappell, K. (2014). The value of RN residency and fellowship programs for Magnet ${ }^{\circledR}$ hospitals. Journal of Nursing Administration, 44(6), 313-314. doi: 10.1097/NNA.0000000000000073

Chattopadhyay, A., Zangaro, G. A., \& White, K. M. (2015). Practice patterns and characteristics of nurse practitioners in the United States: Results from the 2012 national sample survey of nurse practitioners. The Journal for Nurse Practitioners, 11(2), 170-177. doi:10.1016/j.nurpra.2014.11.021

Chen, A. K., Rivera, J., Rotter, N., Green, E., \& Kools, S. (2016). Interprofessional education in the clinical setting: A qualitative look at the preceptor's perspective in training advanced practice nursing students. Nurse Education in Practice, 21, 29-36. doi: http://dx.doi.org/10.1016/j.nepr.2016.09.006 
Chickering, A. W., \& Schlossberg, N. K. (1995). Getting the most out of college. Boston. Clabo L.L. et al. (2015). American Association of Colleges of Nursing. White paper. Reenvisioning the clinical education of advanced practice registered nurses. Retrieved from http://www.aacn.nche.edu/publications/white-papers

Collins, A., Brown, J. S., \& Holum, A. (1991). Cognitive mentorship: Making thinking visible. American Educator, 15(3), 6-11.Retrieved from https://s3.amazonaws.com/academia.edu.documents/39061181/collins_brown_holum_19 91.pdf?AWSAccessKeyId=AKIAIWOWYYGZ2Y53UL3A\&Expires=1509664864\&Sig nature=PxbS7bejiQfrvStr17FgS2O4Tjw\%3D\&response-contentdisposition=inline \%3B\%20filename\%3DCognitive_Apprenticeship_-_making_thinki.pdf

Collins, A., \& Kapur, M. (2015). Cognitive apprenticeship. In R. K. Sawyer, Cambridge handbook of the learning sciences. New York: Cambridge University Press.

Coombs, L. A. (2015). The growing nurse practitioner workforce in specialty care. The Journal for Nurse Practitioners, 11(9), 907-909. doi: http://dx.doi.org/10.1016/j.nurpra.2015.06.014

Creswell, J. (2009). Research design: Qualitative, quantitative, and mixed methods approaches $\left(3^{\text {rd }}\right.$ ed.). Thousand Oaks, CA: Sage Publications.

Creswell, J. W. (2013). Qualitative inquiry and research design: Choosing among five approaches (3rd ed.). Thousand Oaks, CA: Sage Publications, Inc.

Creswell, J. W., Hanson, W. E., Clark Plano, V. L., \& Morales, A. (2007). Qualitative research designs: Selection and implementation. The Counseling Psychologist, 35(2), 236-264. 
doi: $10.1177 / 0011000006287390$

Cusson, R. M., \& Strange, S. N. (2008). Neonatal nurse practitioner role transition: The process of retaining expert status. The Journal of Perinatal \& Neonatal Nursing, 22(4), 329-337. doi: 10.1097/01.JPN.0000341365.60693.39

Dennen, V. P., \& Burner, K. J. (2008). The cognitive mentorship model in educational practice. Handbook of research on educational communications and technology, 3, 425-439. Retrieved from http://faculty.weber.edu/eamsel/Classes/Projects\%20and\%20Research\%20(4800)/Teachi ng\%20and\%20Learning/Dennen\%20(2004).pdf

Dillon, D. L., Dolansky, M. A., Casey, K., \& Kelley, C. (2016). Factors related to successful transition to practice for acute care nurse practitioners. AACN advanced critical care, 27(2), 173-182. doi: 10.4037/aacnacc2016619

Donley, R., Flaherty, M., Sarsfield, E., Burkhard, A., O'Brien, S., \& Anderson, K. (2014). Graduate clinical nurse preceptors: Implications for improved intra-professional collaboration. OJIN: The Online Journal of Issues in Nursing, 19(3). doi: 10.3912/OJIN.Vol198No03PPT01

Dower, C. (2012). Graduate Medical Education. A debate continues over the size and scope of federal subsidies to support residency training of the nation's physicians. Health Affairs, Health Policy Brief. Retrieved from http://www.healthaffairs.org/healthpolicybriefs/brief.php?brief_id=73

Dowling, M. (2007). From Husserl to van Manen. A review of different phenomenological 
approaches. International Journal of Nursing Studies, 44(1), 131-142. doi:

10.1016/j.ijnurstu.2005.11.026

Downey, G., Dalidowicz, M., \& Mason, P. H. (2015). Apprenticeship as method: Embodied learning in ethnographic practice. Qualitative Research, 15(2), 183-200. doi: $10.1177 / 1468794114543400$

Drayton-Brooks, S. M., Gray, P. A., Turner, N. P., \& Newland, J. A. (2017). Building clinical education training capacity in nurse practitioner programs. Journal of Professional Nursing. doi: 10.1016/j.profnurs.2017.02.002

Erikson, C., Hamann, R., Levitan, T., Pankow, S., Stanley, J., \& Whatley, M. (2014). Recruiting and maintaining US clinical training sites: Joint report of the 2013 multi-discipline clerkship/clinical training site survey. Washington, DC: Association of American Medical Colleges.

Fang, D., Li, Y., Arietti, R., \& Bednash, G. D. (2014). 2013-2014 enrollment and graduations in baccalaureate and graduate programs in nursing. Washington, DC: American Association of Colleges of Nursing.

Faraz, A. (2016). Novice nurse practitioner workforce transition into primary care: A literature review. Western Journal of Nursing Research. 38 (11), 1531 - 1545. doi:10.1177/0193945916649587

Farrell, K., Payne, C., \& Heye, M. (2015). Integrating interprofessional collaboration skills into the advanced practice registered nurse socialization process. Journal of Professional Nursing, 31(1), 5-10. doi: http://dx.doi.org/10.1016/j.profnurs.2014.05.006 
Fitzpatrick, S., \& Gripshover, J. (2016). Expert nurse to novice nurse practitioner: The journey and how to improve the process. The Journal for Nurse Practitioners, 12(10), e419. doi:10.1016/j.nurpra.2016.05.012.

Flott, E. A., \& Linden, L. (2016). The clinical learning environment in nursing education: A concept analysis. Journal of Advanced Nursing, 72(3), 501-513. doi:10.1111/jan.12861

Flinter, M., \& Hart, A. M. (2016). Thematic elements of the postgraduate NP residency year and transition to the primary care provider role in a Federally Qualified Health Center. Journal of Nursing Education and Practice, 7(1), 95. doi: 10.5430/jnep.v7n1p95

Forsberg, I., Swartwout, K., Murphy, M., Danko, K., \& Delaney, K. R. (2015). Nurse practitioner education: Greater demand, reduced training opportunities. Journal of the American Association of Nurse Practitioners, 27(2), 66-71. doi: 10.1002/23276924.12175

Gardiner, A., \& Anderson, J. (2013). Cognitive apprenticeship: A key component of contemporary endoscopy education. Gastrointestinal Nursing, 11(7), 45-51. doi:10.12968/gasn.2013.11.7.45

Giddens, J. F., Lauzon-Clabo, L., Morton, P. G., Jeffries, P., McQuade-Jones, B., \& Ryan, S. (2014). Re-envisioning clinical education for nurse practitioner programs: themes from a national leaders' dialogue. Journal of Professional Nursing, 30(3), 273-278. doi: 10.1016/j.profnurs.2014.03.002

Green, J. \& Jackson, D. (2014). Mentoring: Some cautionary notes for the nursing profession. Contemporary nurse, 47(1-2), 79-87. doi:10.1080/10376178.2014.11081909 
Goodman, J., Schlossberg, N. K., \& Anderson, M. L. (2006). Counseling adults in transition: Linking practice with theory. New York: Springer Publishing Company.

Graue, M., Rasmussen, B., Iversen, A. S., \& Dunning, T. (2015). Learning transitions-a descriptive study of nurses' experiences during advanced level nursing education. $B M C$ Nursing, 14(1), 30. doi:10.1186/s12912-015-0080-z

Green, J., \& Jackson, D. (2014). Mentoring: Some cautionary notes for the nursing profession. Contemporary Nurse, 47(1-2), 79-87. doi:10.5172/conu.2013.3528

Griffin, M., \& McDevitt, J. (2016). An evaluation of the quality and patient satisfaction with an advanced nurse practitioner service in the emergency department. Journal for Nurse Practitioners, 12(8), 553-559. doi:10.1016/j.nurpra.2016.05.024

Hallas, D. M., Butz, A., \& Gitterman, B. (2004). Attitudes and beliefs for effective pediatric nurse practitioner and physician collaboration. Journal of Pediatric Health Care, 18(2), 77-86. doi: 10.1016/j.pedhc.2003.09.009

Hallas, D., Biesecker, B., Brennan, M., Newland, J. A., \& Haber, J. (2012). Evaluation of the clinical hour requirement and attainment of core clinical competencies by nurse practitioner students. Journal of the American Academy of Nurse Practitioners, 24(9), 544-553. doi:10.1111/j.1745-7599.2012.00730.x

Harkey, K., Little, S., \& Lazear, J. (2017). The struggle for full practice in North Carolina. The Journal for Nurse Practitioners, 13(2), 131-137. doi: 10.1016/j.nurpra.2016.08.025

Hart, A. M., \& Bowen, A. (2016). New nurse practitioners' perceptions of preparedness for and transition into practice. The Journal for Nurse Practitioners, 12(8), 545-552. 
doi:10.1016/j.nurpra.2016.04.018

Hart, A. M., \& Macnee, C. L. (2007). How well are nurse practitioners prepared for practice: Results of a 2004 questionnaire study. Journal of the American Academy of Nurse Practitioners, 19(1), 35-42. doi:10.1111/j.1745-7599.2006.00191

Heitz, L. J., Steiner, S. H., \& Burman, M. E. (2004). RN to FNP: A qualitative study of role transition. Journal of Nursing Education, 43(9), 416-420. doi: 10.3928/0148483420040901-08

Heinonen, K. (2015). Van Manen's method and reduction in a phenomenological hermeneutic study. Nurse Researcher, 22(4), 35-41. doi:10.7748/nr.22.4.35.e1326

Herman, J., \& Ziel, S. (1999). Collaborative practice agreements for advanced practice nurses: What you should know. AACN Advanced Critical Care, 10(3), 337-342. doi: 10.1097/00044067-199908000-00003

Hevesy, M., Aitchison, R., Ruiz, A., \& Bednar, S. (2016). Nurse practitioners and physician assistants in primary care: An update of changes since 2008. Disease-a-Month, 62(2), 23 36. doi: 10.1016/j.disamonth.2016.01.002

Horner, D. K. (2017). Mentoring: Positively influencing job satisfaction and retention of new hire nurse practitioners. Plastic Surgical Nursing, 37(1), 7-22. doi:10.1097/PSN.0000000000000169

Irwin, C., Bliss, J., \& Poole, K. (2018). Does Preceptorship improve confidence and competence in Newly Qualified Nurses: A systematic literature review. Nurse Education today, 60, 35-46. 
Jacob, S. A., \& Furgerson, S. P. (2012). Writing interview protocols and conducting interviews: Tips for students new to the field of qualitative research. The Qualitative Report, 17(42), 1-10. Retrieved from http://nsuworks.nova.edu/cgi/viewcontent.cgi?article=1718\&context=tqr

Janesick, V. J. (2011). "Stretching" exercises for qualitative researchers (3rd ed.). Thousand Oaks, CA: Sage.

Jangland, E., Yngman Uhlin, P., \& Arakelian, E. (2016). Between two roles - experiences of newly trained nurse practitioners in surgical care in Sweden: A qualitative study using repeated interviews. Nurse Education in Practice, 21, 93-99. doi: http://dx.doi.org/10.1016/j.nepr.2016.10.005

Jarrell, L. (2016). Professional Development and Mentorship Needs of Nurse Practitioners. Journal for Nurses in Professional Development, 32(1), 26-32. doi: 10.1097/NND.0000000000000160

Johnson, R., O'Brien, T., Emerson, S., \& Reed, L. (2017). Perceptions of family nurse practitioner clinical preceptors about usefulness of onsite clinical site visits. Nurse Educator, 42(1), 51-54. doi:10.1097/NNE.0000000000000289

Jones, J., Kotthoff-Burrell, E., Kass-Wolff, J., \& Brownrigg, V. (2015). Nurse practitioner graduates "speak out" about the adequacy of their educational preparation to care for older adults: A qualitative study. Journal of the American Association of Nurse Practitioners, 27(12), 698-706. doi:10.1002/2327-6924.12230

Kelly, J., \& Mcallister, M. (2013). Lessons students and new graduates could teach: A 
phenomenological study that reveals insights on the essence of building a supportive learning culture through preceptorship. Contemporary Nurse: A Journal for the Australian Nursing Profession, 44(2), 170-177. doi:10.5172/conu.2013.44.2.170

Kenny, M., \& Fourie, R. (2015). Contrasting classic, straussian, and constructivist grounded theory: Methodological and philosophical conflicts. The Qualitative Report, 20(8), 12701289. Retrieved from http://nsuworks.nova.edu/tqr/vol20/iss8/9

Keough, L., Arciero, S., \& Connolly, M. (2015). Informing innovative models of nurse practitioner education: A formative qualitative study. Journal of Nursing Education and Practice, 5(5), 88. doi: 10.5430/jnep.v5n5p88

Kaplan, L., Klein, T., Skillman, S., \& Andrilla, C. H. (2016). Faculty supervision of NP program practicums: A comparison of rural and urban site differences. The Nurse Practitioner, 41(7), 36-42. doi:10.1097/01.NPR.0000484321.06426.fa

Kooienga, S. A., \& Carryer, J. B. (2015). Globalization and advancing primary health care nurse practitioner practice. The Journal for Nurse Practitioners, 11(8), 804-811. doi:10.1016/j.nurpra.2015.06.012

Korzon, J., \& Trimmer, W. (2015). The mutual benefits of preceptorship. Kai Tiaki Nursing New Zealand, 21(8), 14-16. Retrieved form https://www.nzno.org.nz/resources/kai_tiaki

Kumaran, S., \& Carney, M. (2014). Role transition from student nurse to staff nurse: Facilitating the transition period. Nurse Education in Practice, 14(6), 605-611. doi: http://dx.doi.org/10.1016/j.nepr.2014.06.002

LeFlore, J. L., \& Thomas, P. E. (2016). Educational changes to support advanced practice 
nursing education. The Journal of Perinatal \& Neonatal Nursing, 30(3), 187. doi: 10.1097/JPN.0000000000000201

Largent, E. A., Grady, C., Miller, F. G., \& Wertheimer, A. (2012). Money, coercion, and undue inducement: a survey of attitudes about payments to research participants. IRB, 34(1), 18. Retrieved from https://www.ncbi.nlm.nih.gov/pmc/articles/PMC4214066/

Larkin, M., \& Thompson, A. (2012). Interpretative phenomenological analysis. Qualitative research methods in mental health and psychotherapy: A guide for students and practitioners, 101-116. Retrieved from http://pureoai.bham.ac.uk/ws/files/10613882/larkin_m_thomson_a_r_IPA_chp08_methods_no_wat ermark.pdf

Latterman, J., \& Merz, J. F. (2001). How much are subjects paid to participate in research? American Journal of Bioethics, 1(2), 45-46. doi:10.1162/152651601300169040

Leggat, S. G., Balding, C., \& Schiftan, D. (2015). Developing clinical leaders: The impact of an action learning mentoring programme for advanced practice nurses. Journal of Clinical Nursing, 24(11-12), 1576-1584. doi:10.1111/jocn.12757

Lindfors, P., Hultell, D., Rudman, A., \& Gustavsson, J. P. (2014). Change and stability in subjective well-being over the transition from higher education to employment. Personality and Individual Differences, 70, 188-193. doi:10.1016/j.paid.2014.06.043

Lipley, N. (2014). Why are there still so few advanced nurse practitioners worldwide?. Cancer Nursing Practice (2014+), 13(8), 9. doi:10.7748/cnp.13.8.9.s10

Logan, B. L., Kovacs, K. A., \& Barry, T. L. (2015). Precepting nurse practitioner students: One 
medical center's efforts to improve the precepting process. Journal of the American Association of Nurse Practitioners, 27(12), 676-682.doi: 10.1002/2327-6924.12265

Lopez, K. A., \& Willis, D. G. (2004). Descriptive versus interpretive phenomenology: Their contributions to nursing knowledge. Qualitative health research, 14(5), 726-735. doi:10.1177/1049732304263638

Lyons, K., McLaughlin, J., Khanova, J., Roth, M., McLaughlin, J. E., \& Roth, M. T. (2017). Cognitive apprenticeship in health sciences education: A qualitative review. Advances in Health Sciences Education, 22(3), 723-739. doi:10.1007/s10459-016-9707-4

Mason, M. (2010, August). Sample size and saturation in $\mathrm{PhD}$ studies using qualitative interviews. In Forum qualitative Sozialforschung/Forum: Qualitative social research, 11 (3). doi:10.17169/fqs-11.3.1428

Matua, G. A., \& Van Der Wal, D. M. (2015). Differentiating between descriptive and interpretive phenomenological research approaches. Nurse Researcher, 22(6), 22-27. doi: $10.7748 / \mathrm{nr} .22 .6 .22 . \mathrm{e} 1344$

MacLellan, L., Levett-Jones, T., \& Higgins, I. (2015). Nurse practitioner role transition: A concept analysis. Journal of the American Association of Nurse Practitioners, 27(7), 389397. doi:10.1002/2327-6924.12165

MacNaughton, K., Chreim, S., \& Bourgeault, I. L. (2013). Role construction and boundaries in interprofessional primary health care teams: A qualitative study. BMC Health Services Research, 13(1), 1-23. doi:10.1186/1472-6963-13-486

Marfell, J., Mc Mullen, P. C., Onieal, M. E., Scheibmeir, M., \& Hawkins-Walsh, E. (2017). Key 
considerations for moving to a nurse faculty role: A dean's perspective. Journal of the American Association of Nurse Practitioners, 29(8), 475-483. doi: 10.1002/23276924.12489

Maten-Speksnijder, A. t., Pool, A., Grypdonck, M., Meurs, P., \& Staa, A. (2015). Driven by ambitions: The nurse practitioner's role transition in Dutch hospital care. Journal of Nursing Scholarship, 47(6), 544-554. doi:10.1111/jnu.12164

Maxwell, J. A. (2013). Qualitative research design: An interactive approach (Vol. 41). Sage publications.

McPherson, G., \& Thorne, S. (2006). Exploiting exceptions to enhance interpretive qualitative health research: Insights from a study of cancer communication. International Journal of Qualitative Methods, 5(2), 73-86. doi: 10.1177/160940690600500210

Meier, S. R. (2013). Concept analysis of mentoring. Advances in Neonatal Care, 13(5), 341-345. doi: 10.1097/ANC.0b013e3182a14ca4

Mentor (2017). In Merriam-Webster's collegiate dictionary. Retrieved from http://www.learnersdictionary.com/definition/mentor

Miles, M. B., Huberman, A. M., \& Saldana, J. (2014). Qualitative data analysis: A method sourcebook (3rd ed.). CA: Sage Publications.

Nielsen, K., Finderup, J., Brahe, L., Elgaard, R., Elsborg, A. M., Engell-Soerensen, V., \& ... Sommer, I. (2017). The art of preceptorship. A qualitative study. Nurse Education in Practice, 2639-45. doi:10.1016/j.nepr.2017.06.009

Norlyk, A., \& Harder, I. (2010). What makes a phenomenological study phenomenological? An 
analysis of peer-reviewed empirical nursing studies. Qualitative Health Research, 20(3), 420-431. doi:10.1177/1049732309357435

Nyhagen, R., \& Strøm, A. (2016). Postgraduate students' perceptions of high-quality precepting in critical care nursing. Nurse Education in Practice, 21, 16-22. doi: http://dx.doi.org/10.1016/j.nepr.2016.09.002

O'Brien, J. L., Martin, D. R., Heyworth, J. A., \& Meyer, N. R. (2009). A phenomenological perspective on advanced practice nurse-physician collaboration within an interdisciplinary healthcare team. Journal of the American Academy of Nurse Practitioners, 21(8), 444-453. doi:10.1111/j.1745-7599.2009.00428.x

O'Leary, Z. (2007). Social change. In O'Leary, Z. The social science jargon buster (pp. 249249). London: SAGE Publications Ltd. doi: 10.4135/9780857020147

Parker, J. M., \& Hill, M. N. (2017). A review of advanced practice nursing in the United States, Canada, Australia, and Hong Kong Special Administrative Region (SAR), China. International Journal of Nursing Sciences, 4(2), 196-204. doi:10.1016/j.ijnss.2017.01.002

Patton, M. Q. (2015). Qualitative research \& evaluation methods (4th ed.). Thousand Oaks, CA: Sage Publications.

Pirret, A. M. (2016). Nurse practitioners' versus physicians' diagnostic reasoning style and use of maxims: A comparative study. The Journal for Nurse Practitioners, 12(6), 381-389. doi:10.1016/j.nurpra.2016.02.006

Poronsky, C. B. (2012). A literature review of mentoring for RN-to-FNP transition. Journal of 
Nursing Education, 51(11), 623-631. doi:10.3928/01484834-20120914-03

Poronsky, C. B. (2013). Exploring the transition from registered nurse to family nurse practitioner. Journal of Professional Nursing, 29(6), 350-358. doi:10.1016/j.profnurs.2012.10.011

Preceptor (2017). In Merriam-Webster's collegiate dictionary. Retrieved from https://www.merriamwebster.com/dictionary/Preceptorship?utm_campaign=sd\&utm_medium=serp\&utm_sour ce $=$ jsonld

Pringle, J., Drummond, J., McLafferty, E., \& Hendry, C. (2011). Interpretative phenomenological analysis: A discussion and critique. Nurse Researcher, 18(3), 2024.doi:10.7748/nr2011.04.18.3.20.c8459

Roberts, S. J., Tabloski, P., \& Bova, C. (1997). Epigenesis of the nurse practitioner role revisited. The Journal of Nursing Education, 36(2), 67-73. doi: 10.3928/0148-4834-19970201-06

Roberts, M. E., Wheeler, K. J., Tyler, D. O., \& Padden, D. L. (2017). Precepting nurse practitioner students: A new view—results of two national surveys of nurse practitioner preceptors. Journal of the American Association of Nurse Practitioners, 29(8), 484-491. doi: $10.1002 / 2327-6924.12482$

Roulston, K., \& Shelton, S. A. (2015). Reconceptualizing bias in teaching qualitative research methods. Qualitative Inquiry, 21(4), 332-342.DOI: 10.1177/1077800414563803

Sabatino, J. A., Pruchnicki, M. C., Sevin, A. M., Barker, E., Green, C. G., \& Porter, K. (2017). Improving prescribing practices: A pharmacist-led educational intervention for nurse 
practitioner students. Journal of the American Association of Nurse Practitioners, 29(5), 248-254.doi: 10.1002/2327-6924.12446

Sargent, L., \& Olmedo, M. (2013). Meeting the needs of new-graduate nurse practitioners: A model to support transition. Journal of Nursing Administration, 43(11), 603-610. doi: 10.1097/01.NNA.0000434506.77052.d2

Schadewaldt, V., McInnes, E., Hiller, J. E., \& Gardner, A. (2013). Views and experiences of nurse practitioners and medical practitioners with collaborative practice in primary health care-an integrative review. BMC family practice, 14(1), 132. doi: 10.1186/1471-2296-14132

Schlossberg, N. K. (1981). Major contributions. Counseling Psychologist, 9(2), 2-5. Retrieved form http://pikespeaksymposium.pbworks.com/f/Schlossberg+Transition+Theory.pdf

Schlossberg, N. (2011). The challenge of change: The transition model and its applications. Journal of Employment Counseling, 48(4), 159-162. doi:10.1002/j.21611920.2011.tb01102.x

Sherbino, J., \& Norman, G. R. (2014). Reframing diagnostic error: Maybe it's content, and not process, that leads to error. Academic Emergency Medicine, 21(8), 931-933. doi: 10.1111/acem.12440.

Siomos, M. Z., Bavis, M. P., Swartwout, K., Danko, K., \& Delaney, K. R. (2016). Nurse practitioner training with the underserved: Building a skilled workforce. The Journal for Nurse Practitioners, 12(2), e37-e43. doi: doi.org/10.1016/j.nurpra.2015.08.034

Sroczynski, M., \& Dunphy, L. M. (2012). Primary care nurse practitioner clinical education. 
Nursing Clinics of North America, 47(4), 463-479. doi:10.1016/j.cnur.2012.08.001

Steiner, S. H., McLaughlin, D. G., Hyde, R. S., Brown, R. H., \& Burman, M. E. (2008). Role transition during RN-to-FNP education. Journal of Nursing Education, 47(10), 441-447. doi:10.3928/01484834-20081001-07

Singh, H., Meyer, A. N., \& Thomas, E. J. (2014). The frequency of diagnostic errors in outpatient care: Estimations from three large observational studies involving US adult populations. BMJ Quality and Safety, 23(9), 727-731. doi: 10.1136/bmjqs-2013-002627

Schumacher, G., \& Risco, K. (2017). Nurse practitioner program curriculum development: A competency-based approach. The Journal for Nurse Practitioners, 13(2), e75-e81. doi: 10.1016/j.nurpra.2016.10.014

Sweeney, C. F., LeMahieu, A., \& Fryer, G. E. (2017). Nurse practitioner malpractice data: Informing nursing education. Journal of Professional Nursing. 33(4), 271-275. doi: 10.1016/j.profnurs.2017.01.002

Sullivan-Bentz, M., Humbert, J., Cragg, B., Legault, F., Laflamme, C., Bailey, P. H., \& Doucette, S. (2010). Supporting primary health care nurse practitioners' transition to practice. Canadian family physician, 56(11), 1176-1182. Retrieved from http://www.cfp.ca

Tavallaei, M., \& Talib, M. A. (2010). A general perspective on role of theory in qualitative research. Journal of International Social Research, 3(11). Retrieved from www.sosyalarastirmalar.com/cilt3/sayil1pdf/tavallaei_abutalib.pdf

Tsekeris, C., \& Katrivesis, N. (2008). Reflexivity in sociological theory and social action. Facta 
universitatis-series: Philosophy, Sociology, Psychology and History, 7(1), 1-12.

Retrieved from http://facta.junis.ni.ac.rs/pas/pas2008/pas2008-01.pdf

Donnelly, F., Wiechula, R., Clancy, M., Tuohy, D., Cooney, A., Dowling, M., ... \& Cassidy, S. (2013). Qualitative data analysis.

Webb, J., Lopez, R. P., \& Guarino, A. J. (2015). Incentives and barriers to precepting nurse practitioner students. The Journal for Nurse Practitioners, 11(8), 782-789. doi: 10.1016/j.nurpra.2015.06.003

Welford, C., Murphy, K., \& Casey, D. (2012). Demystifying nursing research terminology: Part 2. Nurse Researcher, 19(2), 29. Doi:10.7748/nr2012.01.19.2.29.c8906

Wilkes, M., \& Feldman, M. D. (2017). Mentoring clinical trainees: a need for high touch. The Lancet, 389(10065), 135-137. doi:10.1016/S0140-6736(16)32571-5

Wiseman, R. F. (2013). Survey of advanced practice student clinical preceptors. Journal of Nursing Education. 52(5), 253-258. doi:10.3928/01484834-20130319-03

Van Leuven, K. A. (2014). Preparing the next generation of nurse practitioners. The Journal for Nurse Practitioners 10(4): 271-276. doi:10.1016/j.nurpra.2013.12.014

Van der Biezen, M., Derckx, E., Wensing, M., \& Laurant, M. (2017). Factors influencing decision of general practitioners and managers to train and employ a nurse practitioner or physician assistant in primary care: A qualitative study. BMC Family Practice, 18(1), 16. doi: $10.1186 / \mathrm{s} 12875-017-0587-3$

Van Manen, M. (1990). Researching lived experience: Human science for an action sensitive pedagogy. Retrieved from http://ebookcentral.proquest.com 
Van Manen, M. (2017). But is it phenomenology? Qualitative Health Research, 27(6), 775-779. doi:10.1177/1049732317699570

Van Manen, M. (2017a). Phenomenology in its original sense. Qualitative Health Research, 27(6), 810-825.doi:10.1177/1049732317699381 


\section{Appendix A: Demographic Information Form}

Thank you for participating in this study of NP role transition and preceptorship. Prior to our interview (at least 24 hours before the interview day) please complete this form to the best of your ability and e-mail it back to me at viktoriya.pleshkan@waldenu.edu. This will take approximately 7-10 minutes to complete. Thank you.

1. Age: Gender: Highest educational level in nursing?

2. Have you worked in an NP role for at least 3 months (post employer's orientation) and less than 5 years? $\mathrm{Y} / \mathrm{N}$

3. You are practicing as an NP in the U.S.and hold a national NP certification? Y/N

4. You are assigned to a collaborating M.D. (s)? Y/N

5. Have you attended a post-graduation residency program? Y/N

6. Number of years/months in RN role prior to becoming an NP student?

7. In what year have you graduated with your NP (masters)?

8. What is your NP specialty per your board certification? How about your first job specialty? What about your current NP job specialty?

9. Is (was) your first NP job inpatient or outpatient? Current NP job, in or out/patient.?

10. Are you currently practicing in a role of NP (FT), NP(PT), NP(PRN), RN (please choose all that apply)? Other:

11. How many NP jobs have you held since your graduation as an NP?

12. How long was your orientation at your first NP job?

13. Did you have a formal preceptor or mentor assigned to you at your first NP job?

14. After your orientation, did you have an access to a formal preceptor or mentor? If so, was your preceptor or mentor an NP or MD?

15. Do (did) you have an informal mentor at work $\left(1^{\text {st }} \mathrm{NP}\right.$ job)?

16. At your 1 st NP job do (did) you have an office assigned to you or a desk?

17. At your 1st NP job do (did) you have your own schedule/patients or did you see MDs' patients?

18. When you were in NP school, approximately, how many of your preceptors were NPs and how many were MDs?

Notes/comments: 
Appendix B: Interview Protocol

\begin{tabular}{|c|c|c|c|c|c|c|c|c|}
\hline \multicolumn{9}{|c|}{ Interview Protocol Matrix } \\
\hline $\begin{array}{l}\text { Role } \\
\text { Transit } \\
\text { ion } \\
\text { Attribu } \\
\text { te }\end{array}$ & \multicolumn{3}{|c|}{ Prior the graduation } & $\begin{array}{l}\text { Post- } \\
\text { graduation }\end{array}$ & $\begin{array}{l}\text { Content } \\
\text { /baseline } \\
\text { knowledge }\end{array}$ & $\begin{array}{l}\text { Methods } \\
\text { /Teaching } \\
\text { strategies }\end{array}$ & $\begin{array}{l}\text { Sequencing } \\
\text { /complexity }\end{array}$ & $\begin{array}{l}\text { Sociology } \\
\text { /Learning } \\
\text { Environment }\end{array}$ \\
\hline $\begin{array}{l}\text { Intervi } \\
\text { ew } \\
\text { Questi } \\
\text { on (IQ) }\end{array}$ & $\begin{array}{l}\text { Mo } \\
\text { vin } \\
\text { g In }\end{array}$ & $\begin{array}{l}\text { Moving } \\
\text { Through }\end{array}$ & $\begin{array}{l}\text { Moving } \\
\text { Out }\end{array}$ & $\begin{array}{l}\text { Moving in } \\
\text { Again }\end{array}$ & \multicolumn{4}{|c|}{ Cognitive Apprenticeship } \\
\hline \multicolumn{9}{|c|}{ Transition / Role Learning } \\
\hline IQ 1 & $\mathrm{x}$ & $\mathrm{x}$ & $\mathrm{x}$ & $\mathrm{x}$ & $\mathrm{x}$ & & & \\
\hline IQ 2 & $\mathrm{x}$ & $\mathrm{x}$ & $\mathrm{x}$ & $\mathrm{x}$ & & $\mathrm{x}$ & & \\
\hline IQ 3 & $\mathrm{x}$ & $\mathrm{x}$ & $\mathrm{x}$ & $\mathrm{x}$ & & & $\mathrm{x}$ & \\
\hline IQ 4 & $\mathrm{x}$ & $\mathrm{x}$ & $\mathrm{x}$ & $\mathrm{x}$ & & & & $\mathrm{x}$ \\
\hline \multicolumn{9}{|c|}{ Transition / Autonomy and new responsibilities } \\
\hline IQ 1 & $\mathrm{x}$ & $\mathrm{x}$ & $\mathrm{x}$ & $\mathrm{x}$ & $\mathrm{x}$ & & & \\
\hline IQ 2 & $\mathrm{x}$ & $\mathrm{x}$ & $\mathrm{x}$ & $\mathrm{x}$ & & $\mathrm{x}$ & & \\
\hline IQ 3 & $\mathrm{x}$ & $\mathrm{x}$ & $\mathrm{x}$ & $\mathrm{x}$ & & & $\mathrm{x}$ & \\
\hline IQ 5 & $\mathrm{x}$ & $\mathrm{x}$ & $\mathrm{x}$ & $\mathrm{x}$ & & $\mathrm{x}$ & $\mathrm{x}$ & $\mathrm{x}$ \\
\hline \multicolumn{9}{|c|}{ Transition / Reconciling two identities } \\
\hline IQ 6 & $\mathrm{x}$ & $\mathrm{x}$ & $\mathrm{x}$ & $\mathrm{x}$ & $\mathrm{x}$ & $\mathrm{x}$ & $\mathrm{x}$ & $\mathrm{x}$ \\
\hline \multicolumn{9}{|c|}{ Transition /Feelings } \\
\hline IQ 7 & $\mathrm{x}$ & $\mathrm{x}$ & $\mathrm{x}$ & $\mathrm{x}$ & $\mathrm{x}$ & $\mathrm{x}$ & $\mathrm{x}$ & $\mathrm{x}$ \\
\hline
\end{tabular}




\section{Interview Protocol Questions}

\section{Transition /Role Learning}

1. Can you tell me about your experiences with readiness for clinical experiences after graduation and when in school?
2. Can you describe your experiences with learning from your preceptor after graduation and when in school?
3. Can you tell me about your experiences with learning how to care for complex or multiple patients after graduation and when in school?
4. Can you recall and describe your experiences with the clinical sites and communicating with preceptors at your $1^{\text {st }} \mathrm{NP}$ job and when in school?

\section{Transition / Autonomy and new responsibilities}

5. What are/were your experiences with diagnosing and prescribing independently after graduation and when in school?

\section{Transition / Reconciling two identities}

6. What is/was it like for you to be a nurse and a provider combining nursing and medicine in one role after graduation and when in school?

\section{Transition /Feelings}

7. Can you recall and describe what does/did it feel like to be transitioning from an $\mathrm{RN}$ to the role of an NP after graduation and when in school? 
Appendix C: Participants' Demographic Data

\begin{tabular}{|c|c|c|c|c|c|c|c|c|}
\hline Participants \# & 1 & 2 & 3 & 4 & 5 & 6 & 7 & 8 \\
\hline Age & 30 & 31 & 44 & 33 & 35 & 0 & 32 & 31 \\
\hline Gender & $\mathrm{F}$ & $\mathrm{F}$ & $\mathrm{F}$ & $\mathrm{F}$ & $\mathrm{F}$ & & $\mathrm{F}$ & $\mathrm{F}$ \\
\hline How long in NP role (mo.) & 30 & 51 & 13 & 17 & 24 & 17 & 26 & 6 \\
\hline How long in RN role (mo.) & 18 & 29 & 66 & 60 & 24 & 63 & 72 & 36 \\
\hline Graduated with NP (year) & 2015 & 2012 & 2015 & 2016 & 2014 & 2016 & 2014 & 2017 \\
\hline NP specialty (graduated with) & Family & Pediatric & Family & Family & Family & Geriatric & Family & Family \\
\hline NP specialty (1st job) & Family & Pediatrics & Family & $\mathrm{Ob} / \mathrm{Gyn}$ & Retail & Primary Care & Family & Pediatric \\
\hline NP specialty (current) & Family & $\begin{array}{l}\text { Neurosurgery } \\
\text { Pediatrics } \\
\text { neurosurgery ER }\end{array}$ & Hospitalist & $\mathrm{Ob} / \mathrm{Gyn}$ & Occupational & Primary Care & Family & Pediatric \\
\hline NP job setting (1st job) & Outpt & Inpt & Inpt & Outpt & Outpt & Outpt & Outpt & Outpt \\
\hline NP job setting (current job) & Outpt & Inpt & Inpt & Outpt & Outpt & Outpt & Outpt & Outpt \\
\hline NP Employment status & Full time & PRN & Full time & Full time & Part-time & Full time & Full time & Full time \\
\hline Number of NP jobs since graduation & 1 & 2 & 3 & 2 & 5 & 2 & 1 & 1 \\
\hline NP job orientation time (days) & 2 & 90 & 30 & 90 & 14 & 120 & 0 & 7 \\
\hline NP school preceptors (Non-NP) & 1 & 0 & 3 & 2 & 5 & 1 & 2 & 2 \\
\hline NP school preceptors (NPs) & 5 & 5 & 1 & 4 & 0 & 4 & 3 & 4 \\
\hline Number of clinical hours (NP school) & 780 & 0 & 720 & 860 & 500 & 0 & 525 & 750 \\
\hline Own patient schedule (1st NP job) & Yes & No & No & Yes & Yes & Yes & Yes & Yes \\
\hline $\mathrm{RN}$ practice (current) & No & No & No & No & Yes & Yes & No & No \\
\hline Informal preceptor access & Yes & Yes & Yes & No & No & No & Yes & Yes \\
\hline
\end{tabular}




\begin{tabular}{|c|c|c|c|c|c|c|c|c|c|}
\hline Formal preceptor assigned & No & No & & & Yes & Yes & Yes & No & No \\
\hline $\begin{array}{l}\text { Formal preceptor access (post } \\
\text { orientation) }\end{array}$ & No & No & & & Yes & Yes & Yes & No & No \\
\hline Personal office or desk at 1 st NP job & Yes & Yes & & & Yes & Yes & Yes & Yes & No \\
\hline NP school found clinical sites & No & Yes & & & No & No & Yes & No & No \\
\hline Participants \# & & 9 & 10 & 11 & 12 & 13 & 14 & 15 & 16 \\
\hline Age & & 34 & 35 & 31 & 35 & 28 & 0 & 53 & 38 \\
\hline Gender & & $\mathrm{F}$ & M & $\mathrm{F}$ & $\mathrm{F}$ & $\mathrm{F}$ & $\mathrm{F}$ & $\mathrm{F}$ & $\mathrm{F}$ \\
\hline How long in NP role (mo.) & & 48 & 4 & 17 & 17 & 18 & 22 & 17 & 17 \\
\hline How long in $\mathrm{RN}$ role (mo.) & & 17 & 0 & 60 & 240 & 27 & 12 & 324 & 60 \\
\hline Graduated with NP (year) & & 2013 & 2017 & 2016 & 2011 & 2016 & 2015 & 2015 & 2015 \\
\hline NP specialty (graduated with) & & Family & Family & Pediatric & Family & $\begin{array}{l}\text { Adult Gero } \\
\text { primary care }\end{array}$ & Pediatric & Family & Family \\
\hline NP specialty (1st job) & & $\begin{array}{l}\text { Pain } \\
\text { management }\end{array}$ & $\begin{array}{l}\text { Urgent } \\
\text { care }\end{array}$ & $\begin{array}{l}\text { Pediatrics } \\
\text { Behavioral } \\
\text { Health }(\mathrm{BH})\end{array}$ & $\begin{array}{l}\text { Urgent } \\
\text { care }\end{array}$ & $\begin{array}{l}\text { Adult } \\
\text { Cardiology }\end{array}$ & $\begin{array}{l}\text { Pediatric } \\
\text { Primary } \\
\text { Care }\end{array}$ & Orthopedics & Family \\
\hline NP specialty (current) & & $\begin{array}{l}\text { Correctional } \\
\text { medicine }\end{array}$ & $\begin{array}{l}\text { Urgent } \\
\text { care }\end{array}$ & $\begin{array}{l}\text { Pediatrics BH } \\
\& \text { general } \\
\text { Pediatrics }\end{array}$ & GI & $\begin{array}{l}\text { Adult } \\
\text { Cardiology }\end{array}$ & $\begin{array}{l}\text { Pediatric } \\
\text { ER }\end{array}$ & Family & Family \\
\hline NP job setting (1st job) & & Outpt & Outpt & Outpt & Outpt & Inpt and Outpt & Inpt & Outpt & Outpt \\
\hline NP job setting (current job) & & Outpt & Outpt & Outpt & Outpt & Outpt & ER & Outpt & Outpt \\
\hline NP Employment status & & Part-time & Full time & Full time & Full time & Full time & Full time & Full time & Full time \\
\hline
\end{tabular}


Number of NP jobs since graduation

NP job orientation time (days)

NP school preceptors (Non-NP)

NP school preceptors (NPs)

Number of clinical hours (NP school)

Own patient schedule (1st NP job)

RN practice (current)

Informal preceptor access

Formal preceptor assigned

Formal preceptor access (post orientation)

Personal office or desk at 1 st NP job

NP school found clinical sites

$\begin{array}{ll}2 & 2 \\ 14 & 60 \\ 2 & 1 \\ 4 & 3 \\ 0 & 750 \\ \text { Yes } & \text { Yes }\end{array}$

3

3

3

21

4

2

3

700

No

700

Own and MD's

No No

Yes Yes

Yes No

Yes No

Yes No

No Yes
1

3

700

Own and

MD's

No

No

No

No

Yes

No 
Appendix D: Research Flyer

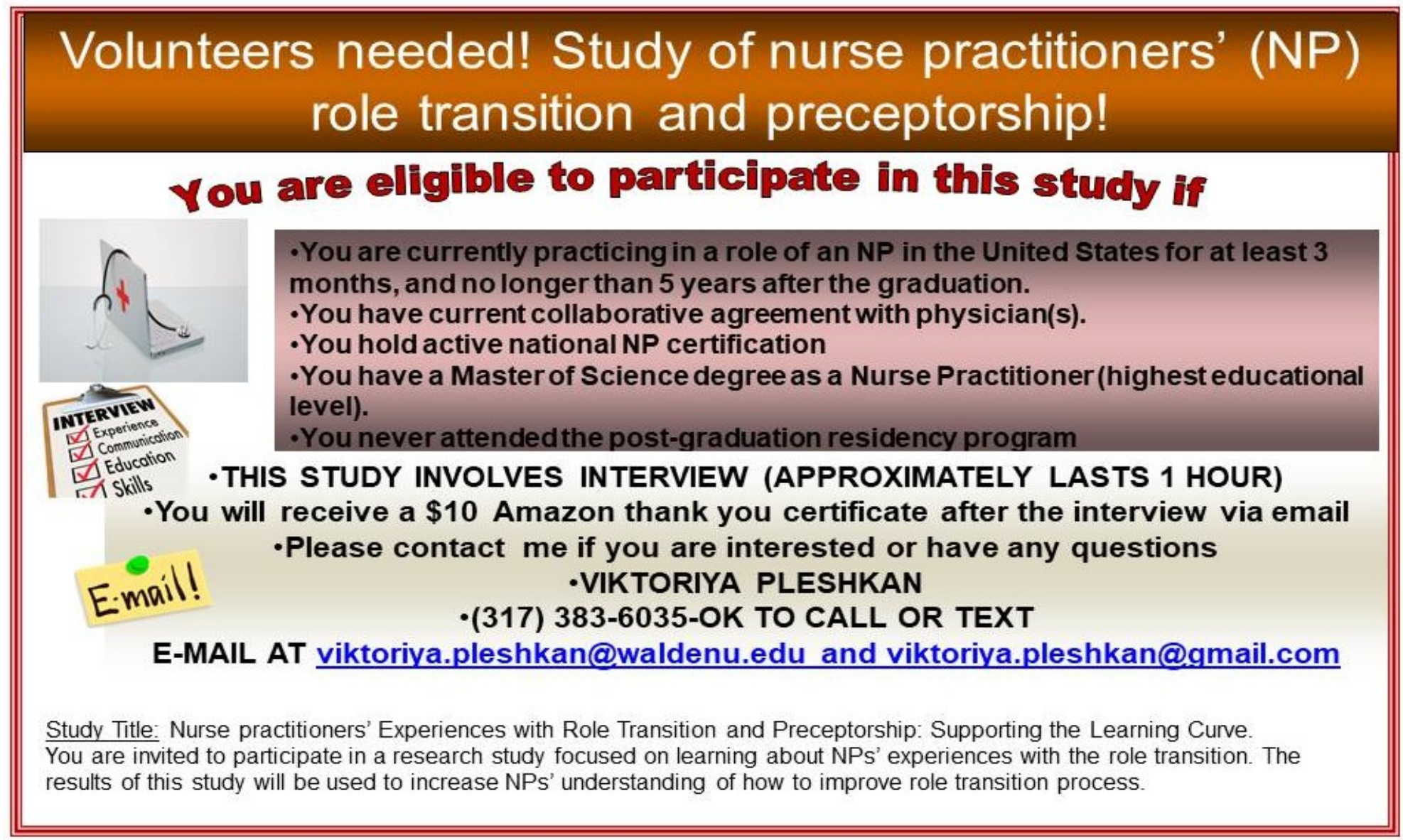


Appendix E

\section{Pre-Graduation Transition Stage Analysis}

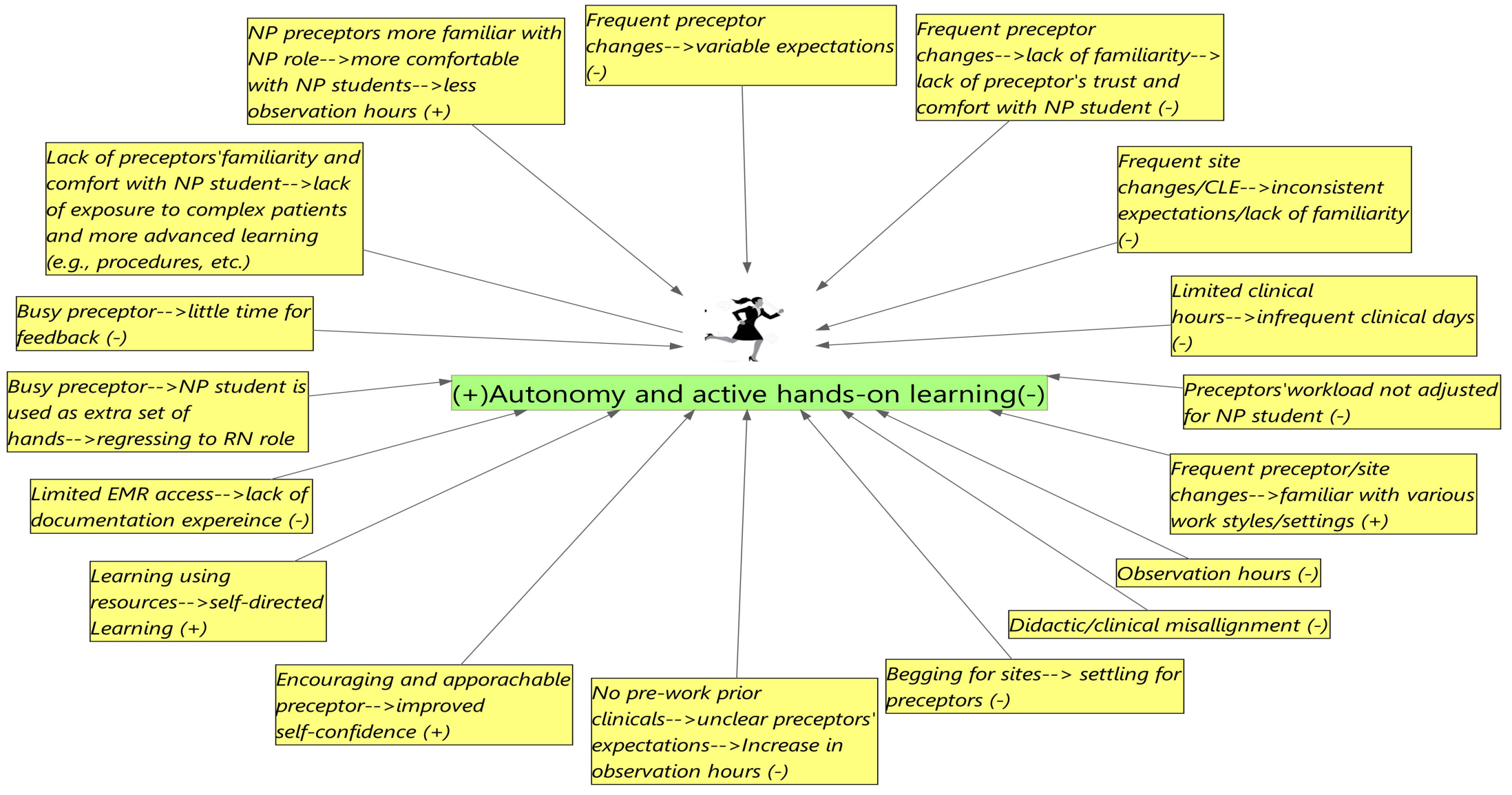

Figure 2. Pre-graduation data analysis and interpretation. (+)-Facilitates. (-)-Impedes. $\rightarrow$ Leads. CLE-clinical learning environment. 


\section{Appendix F}

\section{Post-Graduation Transition Stage Analysis}

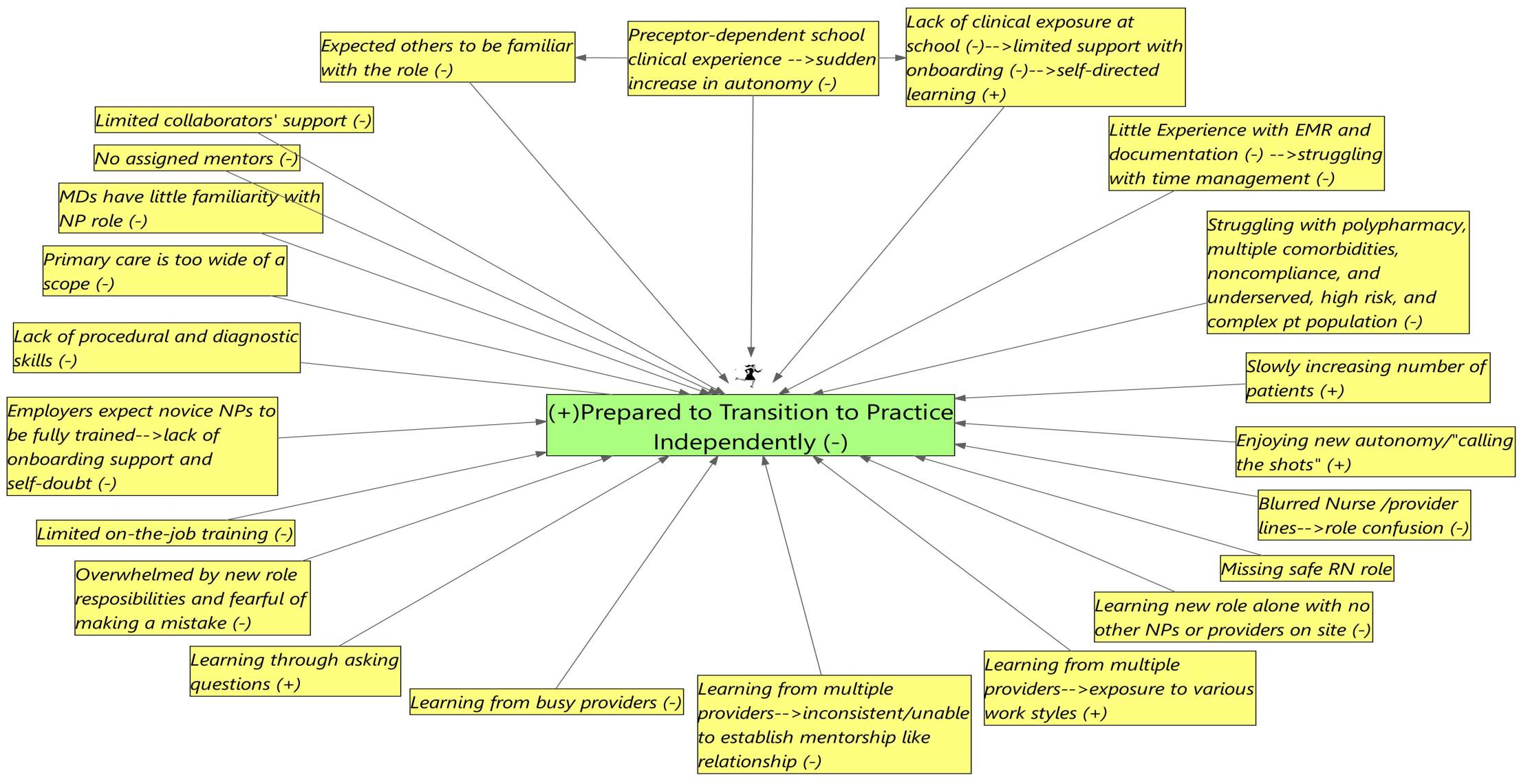

Figure 3. Post-graduation data analysis and interpretation. (+) -Facilitates. (-)-Impedes. $\rightarrow$ Leads. 